$\mathrm{DOE} / \mathrm{BC} / 14600-8$

Distribution Category UC-122

\title{
MODIFICATION OF CHEMICAL AND PHYSICAL FACTORS \\ IN STEAMFLOOD TO INCREASE HEAVY OIL RECOVERY
}

Annual Report for the Period

October 1, 1989 - September 30, 1990

\author{
By \\ Yanis C. Yortsos
}

$\mathrm{DOE} / \mathrm{BC} / 14600--8$

DE91 002234

April 1991

Work Performed Under Contract No. DE-FG22-90BC14600

Prepared for

U.S. Department of Energy

Assistant Secretary for Fossil Energy

Thomas B. Reid, Project Manager

Bartlesville Project Office

P.O. Box 1398

Bartlesville, OK 74005

Prepared by

University of Southern California

Department of Chemical Engineering

Petroleum Engineering Program

Los Angeles, CA 90089-1211

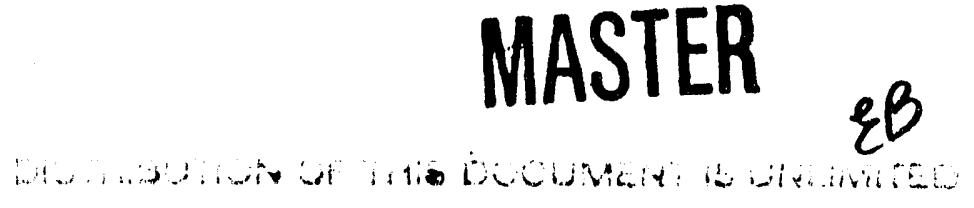




\section{Contents}

$\begin{array}{ll}\text { List of Figures } & \text { vi. }\end{array}$

$\begin{array}{ll}\text { List of Tables } & \text { xi }\end{array}$

$\begin{array}{ll}\text { ACKNOWLEDGEMENTS } & \text { xii }\end{array}$

$\begin{array}{ll}\text { ABSTRACT } & \text { xiii }\end{array}$

1 INTRODUCTION 1

2 IJETWORK MODELS FOR LIQUID-TO-VAPOR PHASE CHANGE AND FLOW IN POROUS MEDIA

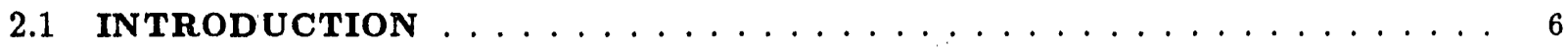

2.2 PHASE EQUILIBRIA IN POROUS MEDIA $\ldots \ldots \ldots \ldots \ldots \ldots$

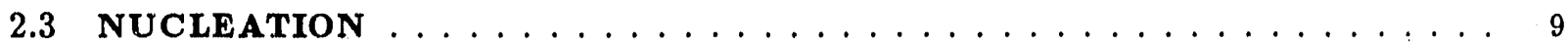

2.4 BUBBLE GROWTH $\ldots \ldots \ldots \ldots \ldots \ldots \ldots \ldots \ldots \ldots$

2.4 .1 Low Superheats $\ldots \ldots \ldots \ldots \ldots \ldots \ldots \ldots \ldots \ldots$

2.4 .2 Moderate and Large Superheats $\ldots \ldots \ldots \ldots \ldots \ldots \ldots$

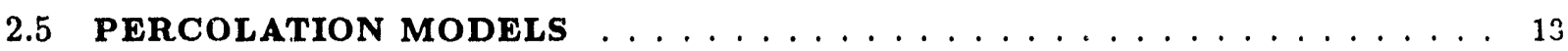

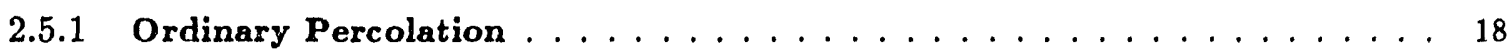

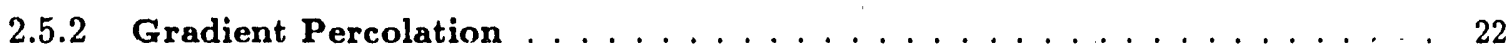

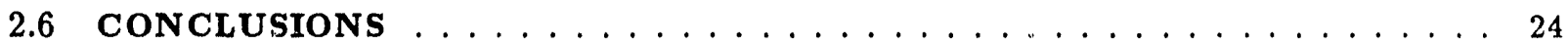

3 STEAM INJECTION VISUALIZATION EXPERIMENTS IN HE UE-SHAW CELLS AND GLASS MICROMODELS

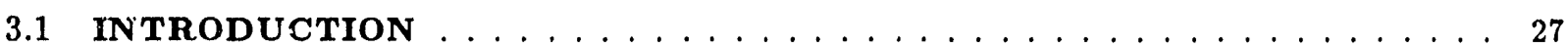

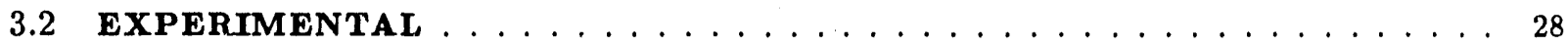

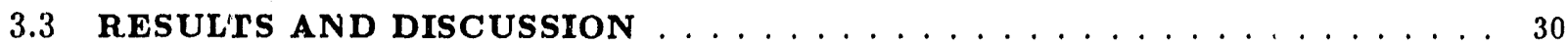

3.3.1 Mechanisms of Displacement in Hele-Shaw Cells $\ldots \ldots \ldots \ldots \ldots$

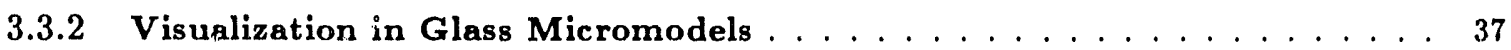

3.4 CONCLUDING REMARKS $\ldots \ldots \ldots \ldots \ldots \ldots \ldots \ldots \ldots \ldots$

4 STEADY-STATE, VAPOR-LIQUID COFCURRENT FLOW: RELATIVE PERMEABILITIES AND END EFFECTS

4.1 INTRODUCTION $\ldots \ldots \ldots \ldots \ldots \ldots \ldots \ldots$ 


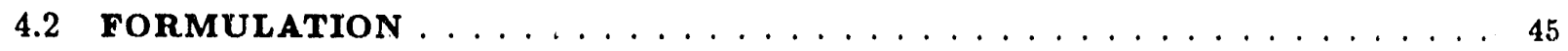

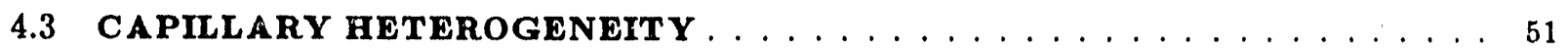

4.4 SATURATION-TEMPERATURE TRAJECTORIES $\ldots \ldots \ldots \ldots \ldots$

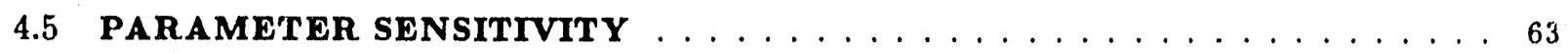

4.6 CONCLUDING REMARKS $\ldots \ldots \ldots \ldots \ldots \ldots \ldots \ldots \ldots$

5 NUMERICAL SYNTHESIS AND SIMULATION OF FRACTURED SYSTEMS US. $\begin{array}{ll}\text { ING FRACTALS } & 77\end{array}$

5.1 INTRODUCTION $\ldots \ldots \ldots \ldots \ldots \ldots \ldots \ldots \ldots \ldots$

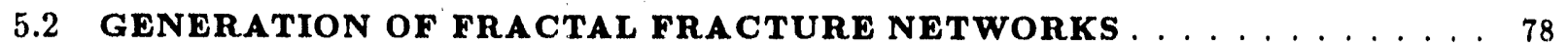

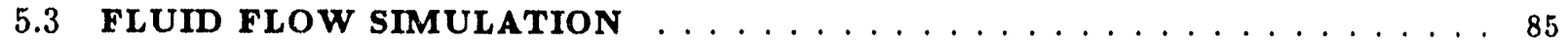

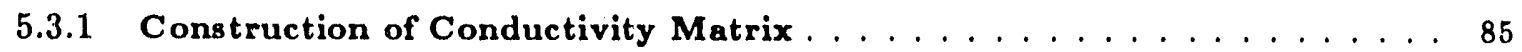

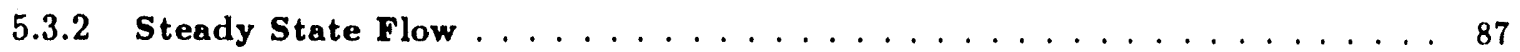

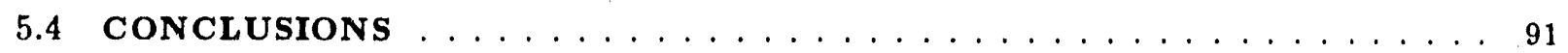

6 LARGE SCALE AVERAGING OF DRAINAGE AT LOCAL CAPILLARY CONTROL 93

6.1 INTRODUCTION $\ldots \ldots \ldots \ldots \ldots \ldots \ldots \ldots$

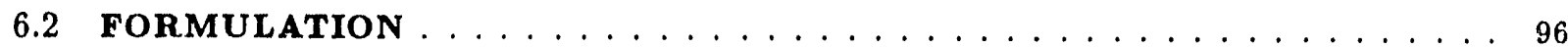

6.3 BEHAVIOR NEAR INVASION THRESHOLD $\ldots \ldots \ldots \ldots \ldots$

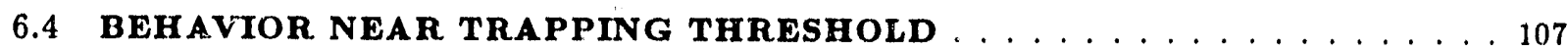

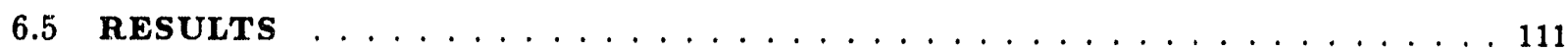

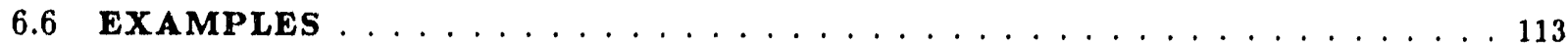

6.7 FLOW PERMEABILITIES $\ldots \ldots \ldots \ldots \ldots \ldots \ldots \ldots \ldots$

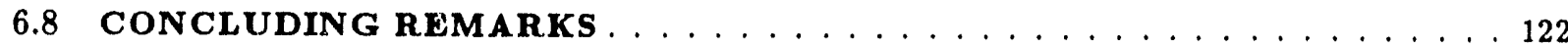

7 LONG WAVES IN PARALLEL FLOW IN HELE-SHAW CELLS

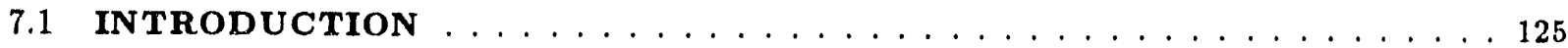

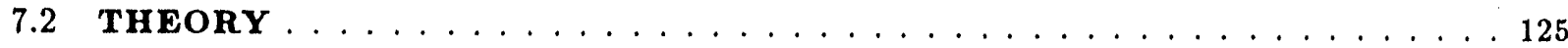

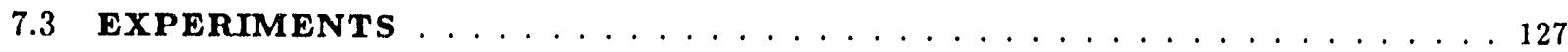

8 MECHANISMS OF FOAM GENERATION AND PROPAGATION IN POROUS MEDIA

8.1 INTRODUCTION $\ldots \ldots \ldots \ldots \ldots \ldots \ldots \ldots \ldots$

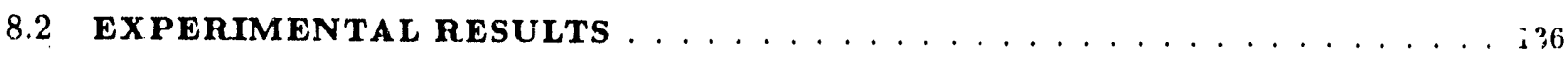


9 FLOW OF NON-NEWTONIAN FLUIDS IN POROUS MEDIA 145

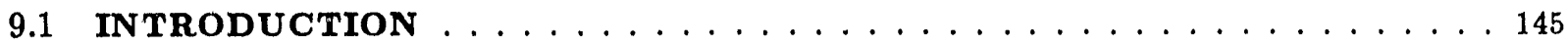

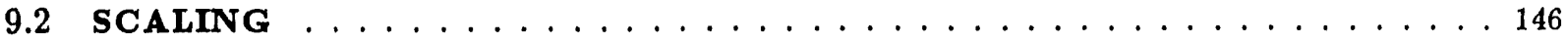

9.3 POWER-LAW FLUIDS $\ldots \ldots \ldots \ldots \ldots \ldots \ldots \ldots \ldots \ldots \ldots$

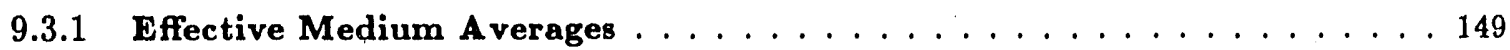

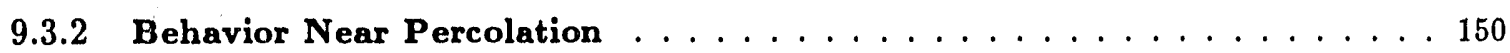

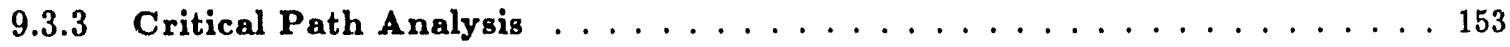

9.4 WEAKLY NON-LINEAR FLUIDS $\ldots \ldots \ldots \ldots \ldots \ldots \ldots \ldots \ldots$

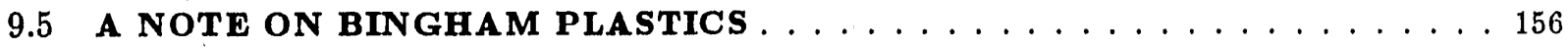

$\begin{array}{lr}\text { SUMMARY AND FUTURE WORK } & 158\end{array}$

$\begin{array}{ll}\text { REFERENCES } & 163\end{array}$

$\begin{array}{lr}\text { APPENDIX } & 170\end{array}$ 


\section{List of Figures}

1 Schematic of a vapor bubble in a porous medium. . . . . . . . . . . 7

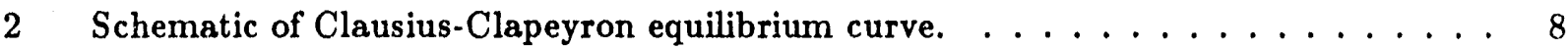

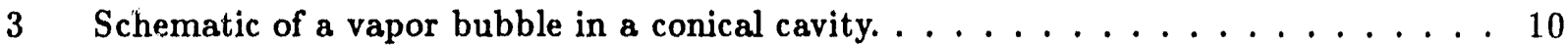

4 Schematic of vapor bubble radius-volume relation in a hydrophobic cavity. . . . . . 11

5 Schematic of single vapor bubble growth in porous medium. . . . . . . . . . . 13

6 Three different stages of vapor growth in the pressure depletion case. . . . . . . . 19

7 Schematics of relative permeabilities as a function of $\beta \ldots \ldots \ldots$

8. Finite volume effect on the depletion of supersaturation and superheat imposed. . . 21

9 The final growth pattern for two different values of $\mu \ldots \ldots \ldots \ldots \ldots$

10 The final growth patterns for two different values of $\mu \ldots \ldots \ldots$

11 Ordinary (a) and gradient (b) percolation growth patterns. . . . . . . . . . 25

12 The modified gradient percolation growth patterns at two values of $\beta \ldots \ldots 26$

13 Glass micromodel with dead ends. . . . . . . . . . . . . . . . . . 28

14 Temperature-shear viscosity relation for Dutrex $739 \ldots \ldots \ldots$

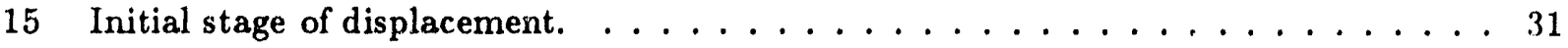

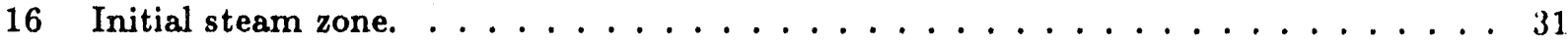

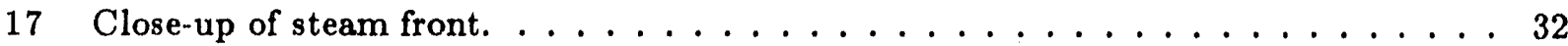

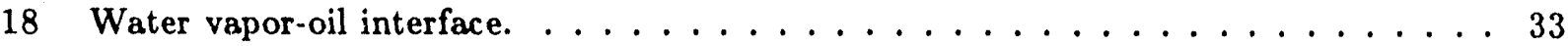

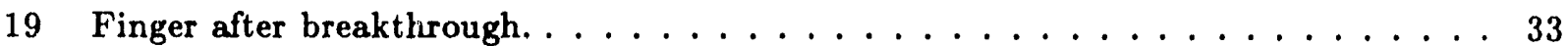

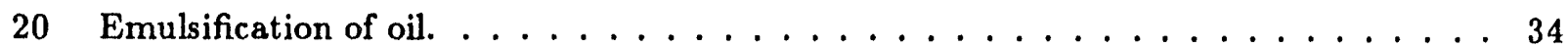

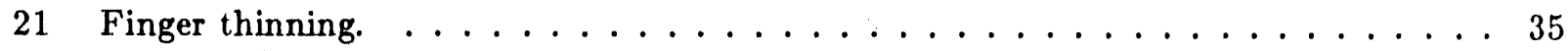

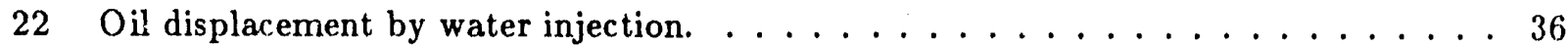

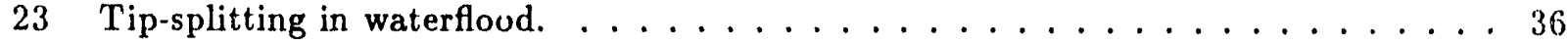

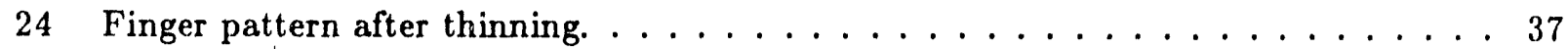

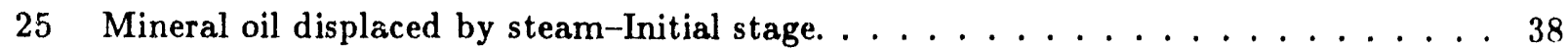

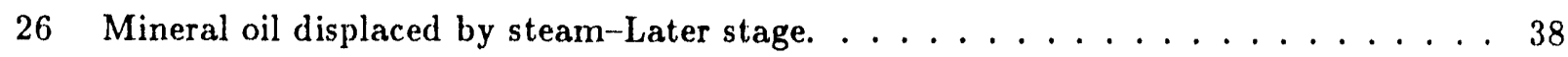

27 Steam injection into glass micromodel. . . . . . . . . . . . . . 39

28 Vapor bubble snap-off. . . . . . . . . . . . . . . . . . . . 40

29 Oil film flow in a dead-end pore. . . . . . . . . . . . . . . 41 
30 Steam injection into a fracture network-Initial stage. . . . . . . . . . . . 41

31 Steam injection into a fracture network-Later stage. . . . . . . . . . . . 42

32 Capillary heterogeneity: saturation and temperature response for a step increase in

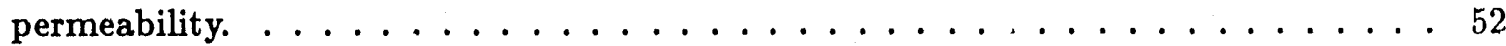

33 Capillary heterogeneity: saturation and temperature response for a step decrease in

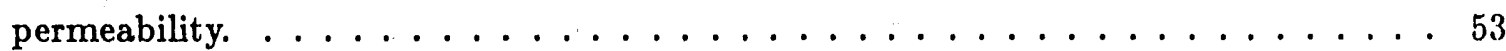

34 Effect of energy injection rate $C$ (btu/hr) on saturation and temperature profiles. . . 55

35 Capillary heterogeneity: effect of conductivity for a step increase in permeability $(\lambda$

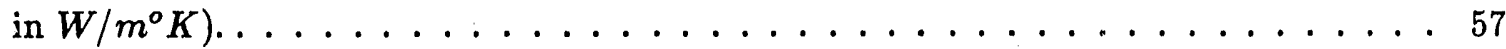

36 Capillary heterogeneity: saturation and temperature response for an fbm permeabil-

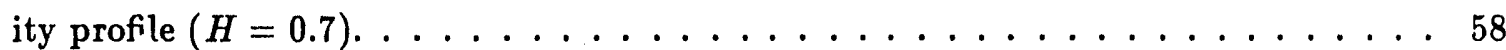

37 Phase portrait and $(T, S)$ trajectories schematic. Arrow indicates upstream direction. 60

38 Phase portrait and $(T, S)$ trajectories for water at conditions of Table 3. Arrow indicates upstream direction. . . . . . . . . . . . . . . 64

39 Phase portrait and $(T, S)$ trajectories for propane at conditions of Miller (1951). Arrow indicates upstream direction. . . . . . . . . . . . . . . 65

40 Phase portrait and $(T, S)$ trajectories for water at conditions of Table 3. Effect of permeability. Arrow indicates upstream direction. . . . . . . . . . . 66

41 Sensitivity of saturation profiles to the rate parameter $R_{m}\left(R_{m}=0.01,1,10\right.$ from

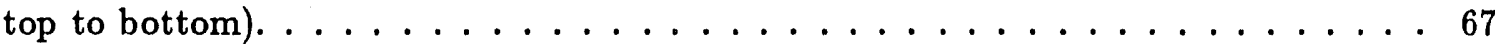

42 Sensitivity of inlet saturation to steam quality and heat injection rate for the condi-

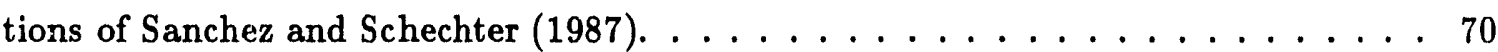

43 Sensitivity of inlet saturation $\left(S_{0}\right)$ to the rate parameter $R_{m}$ for standard conditions: Effect of heat injection rate $C(\mathrm{btu} / \mathrm{hr}) \ldots \ldots \ldots \ldots \ldots \ldots$

44 Sensitivity of error estimate to the rate parameter $R_{m}$ for" standard conditions: Effect

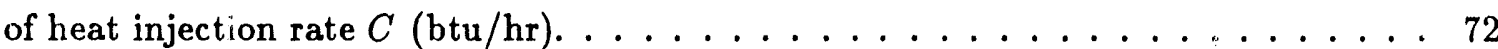

45 Sensitivity of inlet saturation and error estimate to the rate parameter $R_{m}$ for standard conditions and permeability of $0.005 d \ldots \ldots \ldots \ldots \ldots$

46 Sensitivity of inlet saturation and error estimate (at two different magnifications) to the rate parameter $R_{m}$ for conditions of Run 15 in Sanchez and Schechter (1987). . 75 
47 Fractal pattern produced by perpendicular systematic fracturing of an initial square. Six generations of fractures are shown. The initial fracture is parallel to the top and bottom of the map. Fracture widths are exaggerated. . . . . . . . . . . . . . 79

48 Fractal pattern produced by systematic fracturing of an initial square. Nine generations of fractures are shown. The initial fracture is at a given angle with respect to top and bottom of the map. Fracture widths are exaggerated. . . . . . . . . 81

49 Fractal pattern produced by systematic fracturing of an initial square. Six generations are shown. The initial fracture has tapered shape and it is at an angle with respect to top and bottom of the map. Fracture widths are exaggerated. . . . . . . 82

50 Fractal pattern produced by systematic fracturing of an arbitrary quadrilateral medium. Nine generations are shown. Fracture wilths are exagerrated. . . . . . 83

51 The conductivity matrix for one, two and three generations of fractures. . . . . . 88

52 Asymptotic behavior of the total conductivity with increasing number of generations

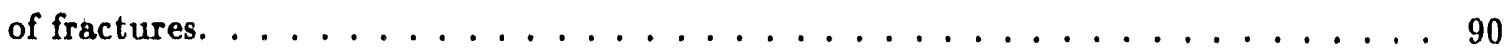

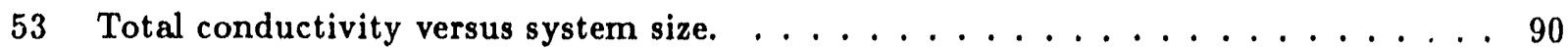

54 Schematic of permeability distribution: (a) Definition of fraction $p$; (b) Definition of fraction $q \ldots \ldots \ldots \ldots \ldots \ldots \ldots \ldots \ldots \ldots \ldots \ldots \ldots \ldots \ldots \ldots \ldots \ldots \ldots \ldots$

55 Schematics of : (a) Individual capillary pressure curves for two permeabilities (in arbitrary units); (b) the $J$ function. . . . . . . . . . . . . . . . . 99

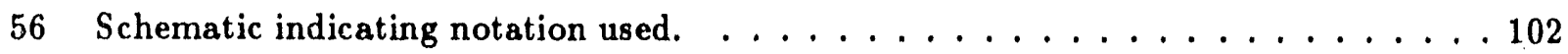

57 Simulation of drainage using Invasion Percolation. Lighter shade corresponds to

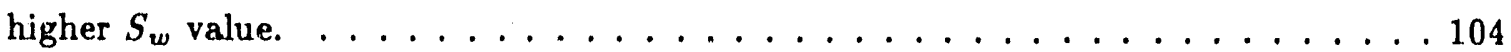

58 Large scale capillary pressure curves obtained by Invasion Percolation in a 3-D cubic lattice. . . . . . . . . . . . . . . . . . . . . . . . . . . . . . .

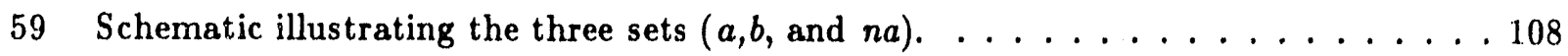

60 Large scale capillary pressure curves obtained by percolation with trapping. . . . . 114

61 Large scale capillary pressure curve for a bimodal distribution, $k_{1}=1, k_{2}=6.25, \rho=$ 0.4 and $\alpha=4$, and for linear $J$ function in a 3-D cubic lattice. Dashed lines indicate

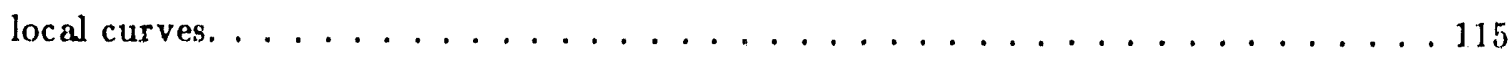

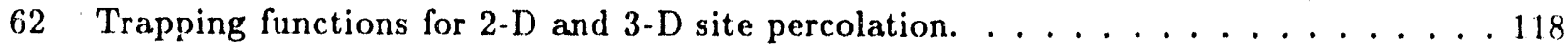




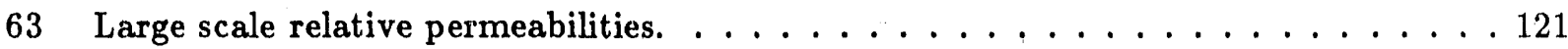

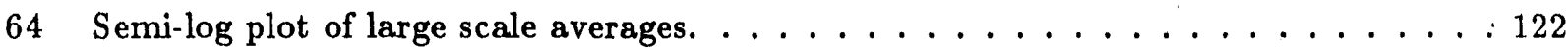

65 Large scale relative permeabilities for Figure 61 using $E M A \ldots \ldots \ldots \ldots$

66 Flow geometry for (a) non-symmetric and (b) symmetric cases. . . . . . . . . 126

67 Single solitary wave. (a) Initial condition, (b) and (c) subsequent stages. . . . . . . 129

$68 \mathrm{Nu}$ nerical simulation of Figure 67. Inset: profile in different scales. . . . . . . . . 131

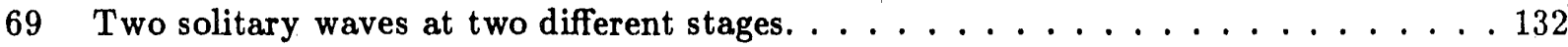

70 Numerical simulation of Figure 69. Inset: profile in different scales. . . . . . . . . . 133

71 Two solitary waves (a) before and (b) after interaction. . . . . . . . . . . 134

72 Numerical simulation of Figure 71 . Inset: profile in different scales. . . . . . . . . 135

73. Pressure drop history in Berea sanstone during simultaneous surfactant and gas

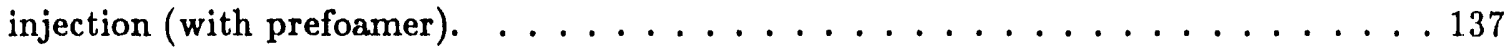

74 Pressure drop history in Berea sanstone during simultaneous surfactant and gas

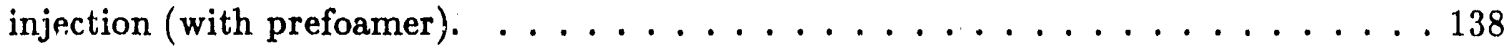

75 Pressure drop history in Berea sanstone during simultaneous surfactant and gas injection (no prefoamer) . . . . . . . . . . . . . . . . . 139

76 Pressure drop history in Berea sanstone during simultaneous surfactant and gas injection (no prefoamer, early time) $\ldots \ldots \ldots \ldots \ldots$

77 Effect of prefoamer in Berea sanstone during simultaneous surfactant and gas injection. 140

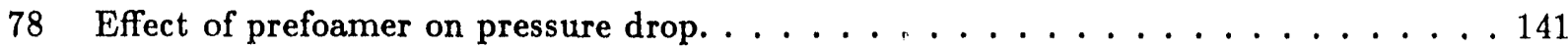

79 Hysteresis effect of pressure drop on gas rate (with prefoamer) . . . . . . . . . 142

80 Hysteresis effect of pressure drop on gas rate (no prefoamer) . . . . . . . . . . . 142

81 Pressure drop measured across four consecutive core sections (upstream to down-

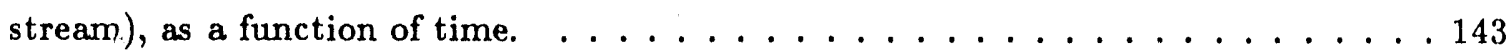

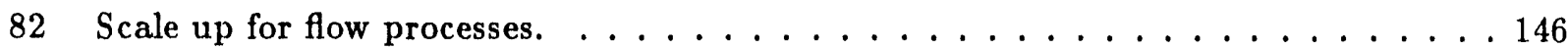

83 Schematics of non-linear pressure drop-flow rate relations (from Scheidegger). . . . 148

84 Schematic of a Bethe Lattice of $z=3$ and a conductance distribution $\ldots \ldots 149$

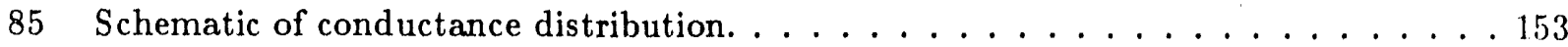

86 Schematic of flow rate-pressure drop relation in the Forcheimer regime. . . . . . . 155

87 Schematic of Bingham plastic mobilization. . . . . . . . . . . . 157 
87 Schematic of Bingham plastic mobilization. . . . . . . . . . . . 157 


\section{List of Tables}

1 Typical parameter values used. . . . . . . . . . . . . . 12

2 List of dimensionless parameters and variables $\ldots \ldots \ldots \ldots$

3 Typical values of variables and parameters for water vapor-liquid flow . . . . . . 49

4 Typical values of variables and parameters for propane vapor-liquid flow . . . . . 50 


\section{ACKNOWLEDGMENTS}

The research reported in this volume was supported by the U.S. Department of Energy under Contract FG19-87BC14126, subcontracted from Stanford University. We would like to express our gratitude to Tom Reid, our DOE Project Manager, for his steady support and commitment. Thanks are also due to Prof. Bill Brigham and Dr. Louis Castanier of SUPRI for their continuing interest and for their invaluable help in administering the subcontract. Part of the work on reservoir heterogeneity was conducted while the author was visiting Universite Pierre et Marie Curie. The work on fractal fracture networks was partly supported by the USC Center for Oil Recovery Research, sponsored by Elf Aquitaine, Oryx, Phillips Petroleum, Shell Development, Unocal and Texaco. All these sources of support are greatfully acknowledged. 


\section{ABSTRACT}

The study of vapor-liquid flow in porous media continued. Three aspects were addressed: (i) Extension of our previous vapor-liquid model for solution gas-drive to a water liquid-water vapor (steam) system in a pore network; (ii) Visualization of steam injection in Hele-Shaw cells and glass micromodels; and (iii) Macroscopic description of concurrent vapor-liquid flow in porous media.

We used the methodology previously developed for a solution gas-drive process to model the phase change and phase growth during a vaporization process driven by a temperature increase. These phenomena are typically encountered in steam injection processes. The model includes nucleation, temperature and heat flux rates, and pore statistics (geometry and topology), but neglects temperature gradients. To include the la,tter, a variation of gradient percolation was proposed. Conditions are developed that place constraints on the heating rate for the applicability of percolation theory.

Visualization experiments were initiated during the period reported for the study of steam injection in Hele-Shaw cells and in glass micromodels. Initial experiments were cunducted at low pressures. Results obtained in Hele-Shaw cells demonstrate the interplay between steam injection, steam condensation, viscous fingering, heat transfer and steam distillation effects. A non-Newtonian response of the displaced synthetic oil, Dutrex 739, was also observed. Results in glass micromodels showed the importance of thin films, surface roughness and three-phase flow for the recovery of oil from dead-end pores. Both experiments revealed that steam fronts are not stationary but undergo constant rearrangements as a result of condensation and injection.

The macroscopic modeling of concurrent, steam-water (and, in general, vapor-liquid) flow was also considered. Experiments at steady-state conditions are routinely performed to measure relative permeabilities of single component vapor-liquid systems, such as steam-water. Even though they are performed under adrabatic conditions, the interpretation of results has proved to be a difficult task because of phase charige, heat transfer, capillarity and rate considerations. We have undertaken a systematic study of the saturation and temperature distributions in steady-state, vapor-liquid flows and examined the sensitivity to parameters such as injection rate, permeability and core length. Two sets are analyzed, one pertaining to the simultaneous injection of a two-phase mixture and another involving phase change (liquid-to-vapor) within the core. End effects and, in general, effects associated with capillary heterogeneity are analyzed in detail. 
Significant progress was made in the study of reservoir heterogeneity and its effects on flow processes. We have considered three general areas: (i) The representaticn of naturally fractured systems; (ii) The large-scale averaging (derivation of pseudo-functions) for displacement in macroscopically he'erogeneous systems; and (iii) The study of parallel flow, typically encountered in long and narrow reservoirs.

Present models for the representation of naturaliy fractured systems rely on the double-porosity Warren-Root model or on random arrays of fractures. These models fail to capture the self-similarity of the fracture network. We investigated the application of fractal geometry for the numerical construction of the network of sractures in a naturally fracturcd system. The system obtained exhibits self-similar behavior at all scales. Numerical methods were developed for the simulation of steady state, single-phase flow in the network. To facilitate the solution of the problem, a specific formulation is devised that takes account of the fractal nature of the system. It is found that the total permeability is dependent on system size following a power-law, consistent with fractal geometry concepts.

Large scale averaging is important for the description of displacement processes in heterogeneous porous media. For example, in immiscible displacement, a key objective is the determination of effective (pseudo) capillary pressure and relative permeabilities. Present models are intrinsically unable to provide the local saturation distribution, which is needed for the computation of effective flow properties. Paralleling pore-level approaches, we propose a percolation method for the derivation of large scale properties in a drainage process at low flow rates. Percolation concepts are applied to a macroscopically heterogeneous region with a random and uncorrelated permeability distribution. We show that at conditions of local capillary control, the large scale capillary pressure curve is a non-trivial average of the individual curves. Large scale capillary trapping is predicted and a corresponding large scale trapped saturation is calculated. Large scale phase premeabilities are also derived. It is found that capillary heterogeneity renders a system more strongly wet in a macroscopic sense.

Fluid flow in Hele-Shaw cells and porous media has been a subject of great interest. The majority of studies so far have focused on frontal displacement, particularly on viscous fingering instabilities. Little or no attention has been paid to the motion of lateral interfaces, which are parallel to the mair flow direction. While lacking the richness of frontal displacement, parallel flow 
is, nonetheless, an interesting and often encountered regime. We have conducted studies in our laboratory which show that such interfaces in Hele-Shaw cells support wave motion, the dynamics of which are similar to shallow water waves. In particular, we have found evidence that, at least for a single interface, long waves can be described by the KdV equation, which, importantly, predicts the formation of solitons. Such solitary waves were observed in our experiments in Hele-Shaw cells.

The third area of research in this report involves chemical additives for the improvement of recovery efficiencies. We have been studying the following three aspects: (i) Caustic additives at elevated temperatures; (ii) Foam generation; and (iii) Non-Newtonian flow in porous media. The study of canstic injection a: elevated temperatures, specificaliy tifie silica dissolution and caustic consumption, has been terminated. A technical report will summarize the results obtained. Here, we address the remaining aspects (ii) and (iii).

Experiments were conducted involving the simultaneous, steady-state injection of nitrogen gas and surfactant solution in Berea sandstone cores in order to study foam generation and its dependence on various parameters. Pressure taps at regular intervals along the core allowed for the monitoring of the pressure profiles. Incubation phenomena were consistently observed in all experiments. Injection rate, injection quality, and core length were found to infiuence most significantly the incubation period. Based on the in-situ pressure measurements, the propagation of foam in the core was also inferred. End effects and heterogeneity, in general, are suspected to play an important role in these processes. We currently analyze present models for the interpretation of the results obtained.

A final investigation involved the study of the flow behavior of non-Newtonian fluids in porous media. We have examined certain aspects relevant to power-law fluids, weakly non-linear fluids and Bingham plastics. For the first two cases, we developed expressions for the calculation of the single-phase flow properties, including an effective medium approximation and a percolation approach. Aspects of two-phase flow were also analyzed for power-law and Bingham fluids. In the former case, emphasis was placed on relative permeability scaling, while for the latter we focused on mobilization conditions. 


\section{INTRODUCTION}

This report covers work performed in the area related to physicochemical factors for the improvement of oil recovery efficiency in steamfloods. Specific goals of the original subcontract were the further development of a chemical-steam simulator, the study of the mechanisms of vapor-liquid flow, the effect of foaming agents on oil displacement and the effect of caustic solutions at elevated temperatures. Upon renewal of the suljcontract on February 22, 1990, additional objectives were added involving studies of heterogeneity and non-Newtonian flow.

A cheinical-steam simulator has been partly developed over a period of several years with the support of previous DOE contracts to USC. A renewed effor had started in the last quarter of the previous reporting period to further debug and upgrade the simulator. New features were added in three-phase relative permeabilities, the vertical equilibrium and the phase behavior subroutines. The simulator consists of several thousand Fortran statements in 13 files, each containing several subroutines. However, due to the complexity of the task, progress was unacceptably slow. At present, we have halted work in this area.

The study of vapor-liquid flow in porous media continued in an effort to understand the basic mechanisms involved in vapor-liquid flow, phase change and transport. Despite the success and the widespread use of steam injection, a good understanding of the process is still lacking. The state of the art is far less satisfactory than in standard immiscible processes, for example drainage. The same also applies to other processes involving phase change in porous media, such as solution gasdrive. The underlying reason is that phase change is driven by supersaturation in temperature (or concentration) and phase growth requires the transport of heat (or mass). This introduces a host of new variables and parameters absent from standard immiscible displacement, and issues such as rate of heating (or pressure decline) become important. However, for lack of a better approach, methods currently used still rely on conventional process representation.

Realizing this inadequacy, we have concentrated over the past two years on the understanding of vapor-liquid phase change and flow. Because of its relative simplicity, our first effort was directed to the analysis of the solution gas-drive process, where growth occurs by pressure depletion and transport occurs only through the pore space. The study (Yortsos and Parlar [106]) identified a rich variety of interesting phenomena, including nucleation, mass transfer, inertia and viscous effects. Our next step was to apply the same methodology to the vapor-liquid flow problem, where an 
increase in temperature drives the process. The approach is outlined in Chapter 2 of this report. Although a significant improvement over previous studies, there are still various shortcomings, for example the heat transfer is not adequately represented. Partial remedies in the presence of a temperature gradient are presented.

To compare the theoretical predictions with experiments, but also to enhance our understanding of the steam injection process, where displacement of an oleic phase is involved, flow visualization was conducted in transparent, pyrex glass Hele-Shaw cells and micromodels. Interestingly, such experiments have not been previously reported. In fact, to our knowledge, this is the first time that visualization studies for steamfloods in geometries pertinent to porous media have been undertaken. As expected, a variety of interesting phenomena were observed, as discussed in Chapter 3. Of particular relevance are the issues of steam front dynamics, the displacement of heavy oil and the importance of viscous fingering, steam distillation effects, etc. A partial resolution is offered in Chapter 3.

In a related study that complements our previous work on counter-current flows (Satik et al., [81]), we addressed in detail the steady-state, adiabatic vapor-liquid concurrent flow in porous media. Here, use is made of the conventional formalism, and the objective is to determine the sensitivity of relative permeability methods to parameters, such as rate. The need for a more detailed description is underscored by the wide scatter of experimental data in the derivation of relative permeabilities of steam-water systems. A number of variables, such as steam quality, mass and enthalpy injection rates, pressure, permeability and system length, all affect significantly the process. In fact, the vapor-liquid saturation profiles, even under adiabatic conditions, are expected to differ from ordinary profiles in gas-liquid flow. This study is presented in Chapter 4. It should be pointed out that this work also makes use of our recent work on capillary heterogeneity in immiscible displacement (Yortsos and Chang, [105]).

The second main aspect of our research addresses the role of heterogeneity in the performance of thermal methods. This approach is on the macroscopic level, pore-scale heterogeneity being investigated in the previous chapters. We have identified three topics that have direct relevance to displacement processes, in gexieral, namely fractured systems, large-scale averaging and parallel flow. The importance of frastured reservoirs vis-a-vis heavy oil can hardly be overstated. Largescale averaging is essential for the derivation of pseudo-functions for coarse-grid simulation, while 
parallel flow (also known as the Vertical Flow Equilibrium assumption) occurs frequently in typical geologic formations.

Current methods for the representation of naturally fractured systems are minor modifications of the original Warren-Root model. Such models necessitate matrix blocks of a fixed length scale, thereby failing to capture the property of statistical self-similarity that appears to characterize many naturally fractured systems. Recent field observations by Barton (1989) have confirmed this important property in a veriety of fractured rocks [7]. Self-similarity implies the absence of a characteristic length scale. Instead, a cascade of scales (fractures of variable size) at different generations exist, which attribute to the system properties which can best be described by fractal geometry. We have applied fractal geometry techniques by adapting the methods described in Barnsley (1988) to synthetically construct the network of fractures. Such objects are novel, and have properties that differ from those of homogeneous rocks. For instance, permeability is scaledependent, as discussed in Chang and Yortsos (1990), and the representation of fluid flow and transport is substantially different, as revealed by numerical simulations. This subject is discussed in Chapter 5.

Large-scale averaging is important for the description of displacement in macroscopically heterogeneous media. Among the key objectives is the derivation of pseudo-functions (e.g. capillary pressure, relative permeabilities, et. . . Present continuum models use volume averaging of the equations that describe the process locally, in order to arrive at larger-scale results that contain grid-scale heterogeneity. Unfortunately, all these methods are intrisically defficient, because of their inability to provide the local saturation distribution which is needed for proper averaging. The problem is, in many ways, similar to the pore-level problem of obtaining relative permeabilities and other flow properties in terms of the pore structure. It is, therefore, possible that such methodologies can be successfully applied to treat the heterogeneity at the larger scale. Chapter 6 outlines the application of percolation theories in their various forms for the derivation of fundamentally proper pseudo-functions for a drainage process.

The third topic in reservoir heterogeneity deals with processes under parallel flow conditions. By this we imply long and narrow reservoirs, with large aspect ratio, or, more formally, where the dimensionless parameter $R=L / H\left(k_{v} / k_{h}\right)^{\frac{1}{2}}$ is large. It has been demonstrated that such conditions are indeed prevalent in many geological systems. The simplest experimental configuration involves 
the parallel flow of two immiscible fluids in a Hele-Shaw cell. A preliminary analysis indicates that at steady-state, the fluid interface is flat and parallel to the main flow direction, at least for high enough capillary numbers, although this may sot necessarily be the case for flow in a porous medium. Incidentally, such steady-state flows in laboratory cores are often used for the determination of steady-state relative permeabilities. We have proceeded with the study of the interface dynamics in a narrow and lo.ag Hele-Shaw cell. The results of the study, ! oth theoretical and experimental, are summarized in Chapter 7. As anticipated from the theory, solitary waves were experimentally observed. This is the first such reported application of solitons in porcus media flows.

Finally, a major component of our research involves the study of chemical additives for purposes of EOR and mobility control. Past work in our group dealt with the application of alkaline injection at elevated temperatures. This investigation has been completed, although compiling of the report and analyzing the data has been slower than anticipated. We hope that a final report on the subject world be available in the near future. In parallel, we have been conducting experimental work on foam formation and propagation in laboratory cores. On the theoretical side, last year's efforts concentrated on the analysis of non-Newtonian flow in porous media.

Despite the significant advances on the understanding of foam generation in porous media, the state of the art is still incomplete. Particularly lacking are experimental data on foam flow in laboratory cores, that would serve to bolster or resine existing theories. Continuing our previous study, we experimented with the simultaneous injection, at constant rates, of nitrogen gas and surfactant solution, with the objective to monitor the rate of foam formation and foam propagation at different locations within the porous medium. Pressure transducers were used for this purpose. Injection rates, core length, gas fraction and the use of a prefoamer are relevant variables that affect foam properties. Of particular importance to our investigation is the understanding of the incubation period that has elapsed before the generation of strong foam, as well as the foam propagation rates. The study is briefly described in Chapter 8.

A final issue related to chemical additives involves the modeling of non-Newtonian fluid flow in porous media. We note that most EOR fluids, including foams, exhibit non-Newtonian behavior. The effect of microstructure on the flow rate-pressure drop relations is well known for Darcy flow of Newtonian fluids. Because of their linear behavior at the low flow rate conditions in porous 
media, the latter can be analyzed relatively easily. However, this is not the case for non-Newtonian flows in porous media. Not surprisingly, the state of the art is far from complete in this area. A particularly important question is how does the pore structure affect the macroscopic flow behavior, and, thus, the macroscopic models. Answers to this complicated question can be found in certain simple cases, for example in power-law fluids. We point out that bulk foam flow in capillaries has been approximated by a power-law expression. We analyse this and related issues in Chapter 9.

Although some of the ahove studies may not be immediately related to heavy oil recovery, they do have direct potential applications in this area. Fractured systems, large-scale averages, parallel flow conditions, and non-Newtonian flow are subjects that play a central role in heavy oil recovery. As an example, one could mention the recovery of a Bingham plastic-like heavy oil from naturally fractured systems, in relatively long and narrow reservoirs, and the need for its simulation. Unfortunately, our understanding of these areas is not very satisfactory. The fundamental research outlined in the subsequent chapters is aimed to improve this condition. This is necessary not only for the specific objective of heavy oil recovery, but also for more general oil recovery processes. 


\title{
2 NETWORK MODELS FOR LIQUID-TO-VAPON PHASE CHANGE AND FLOW IN POROUS MEDIA
}

\author{
C. Satik and Y.C. Yortsos
}

\subsection{INTRODUCTION}

Vapor-liquid flow in porous media involves diverse phenomena such as phase quilibria, phase change and phase growth. Its dynamics are controlled by an externally imposed supersaturation. The phase change process can be in the form of vaporization or condensation (related to drainage or imbibition, respectively). In vaporization processes, the supersaturation is imposed by a depletion of the liquid pressure, as in solution gas-drive and in cavitation, or by a temperature increase, as in boiling in porous media. Condensation processes can be characterized similarly. Applications abound in solution gas-drive, steam injection, geothermal systems, boiling and cavitation processes.

The process of phase change in the bulk is well understood ([66], [67], [83]). However, little progress has been made about phase change in porous media. Yortsos and Parlar [106] recently studied the basic mechanisms involved in the case of solution gas-drive, which involves two components, one of which is volatile. They examined issues of nucleation, supersaturation and gas phase growth. They have found that phase (or bubble) growth in porous media can be stabilized and :ontrolled by pore wall curvature and geometry, and by pore space connectivity. Furthermore, they suggested a percolation model for growth under conditions of low supersaturation values.

Processes involving phase change, although they may differ in the way the supersaturation is imposed (pressure depletion or temperature increase), are similar in many other ways, because they share many common aspects, such as inertia, viscous and surface forces. In addition, bubble growth is affected by molecular transport, e.g. by diffusion of the volatile component in the case of pressure depletion (solution gas-drive), or by conduction of heat in the case of temperature increase (boiling). In this chapter, our emphasis will be on liquid-to-vapor phase change, driven by temperature differences.

Theoretical work to model such flows at the micro-level has been limited. Besides the popular to the geothermal literature, but otherwise unsupported, model of straight line relative permeabilities, the only work to address such issues was undertaken by Parlar and Yortsos [60], and by Yortsos and Parlar [106], in the context of steam-water, and solution gas drive, respectively. Both papers are 


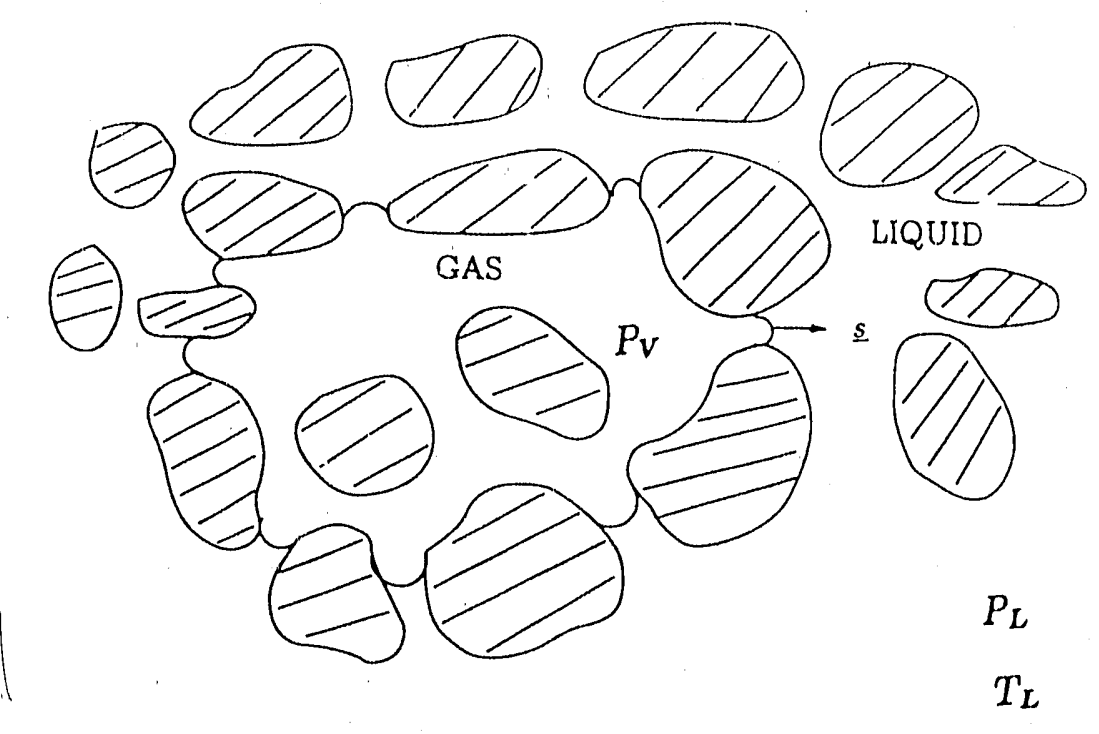

Figure 1: Schematic of a vapor bubble in a porous medium.

restricted to the quasi-static limit, however, where temperature and pressure gradients are small, thus relative permeabilicies are only saturation (and history) dependent. It must be noted that the model of Parlar and Yortsos [60] for drainage does indeed corroborate the findings of Sanchez and Schechter [78]. Nevertheless, additional work is needed to support this result and to resolve other issues. This is partly attempted here. As in Yortsos and Parlar [105], an analysis of phase equilibria, nucleation and bubble growth is necessary. The characteristic differences will be pointed out. Two general models are discussed, one in which spatial temperature gradients are negligible and the heating is uniform, and another in which heat transfer dominates the distribution of phases. Although progress is made in both directions, significant work still remains to be done.

\subsection{PHASE EQUILIBRIA IN POROUS MEdia}

To study phase equilibria we consider a vapor bubble at equilibrium with a liquid in a porous medium (Figure 1). We assume that nucleation has ceased and that individual interface menisci are spherical (although macroscopic growth is certainly not spherical). At equilirium, temperature, liquid and vapor pressures are all spatially uniform. Thermodynamics dictate that the Laplace 


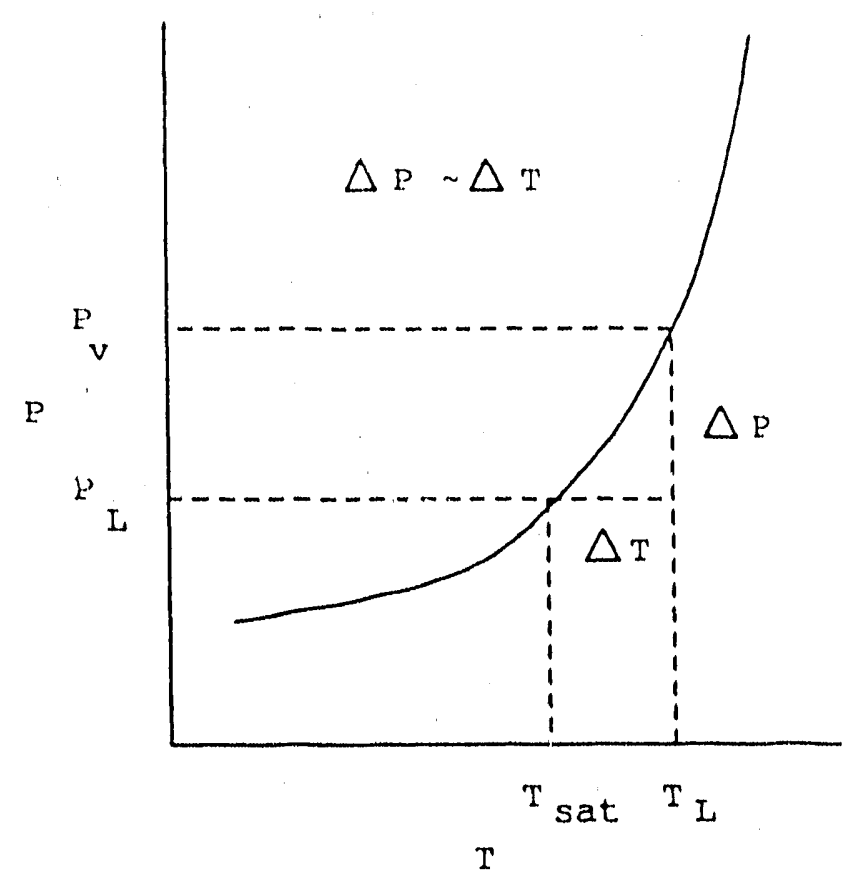

Figure 2: Schematic of Clausius-Clapeyron equilibrium curve.

equation is satisfied at any point, hence the radius of curvature of any vapor-liquid meniscus is

$$
P_{V}-P_{L}=\frac{2 \gamma}{r}
$$

where $\gamma$ is the interfacial tension. For simplicity, we neglect Kelvin effects and assume that the pressure in the vapor phase $P_{V}$ is equal to the saturation vapor pressure at $T_{L}$, as given by the Clausius-Clapeyron equation

$$
P_{V}=P_{V O}\left(T_{L}\right)
$$

Phase equilibria in a porous medium may be schematically interpreted as in Figure 2. The system is initially in the liquid state in equilibrium at pressure $P_{L}$ (point A). Upon heating, the liquid temperature is raised uniformly at constant pressure. Until the equilibrium curve is reached (point B) no phase change occurs. Further increase in temperature creates the superheat $\Delta T=T_{L}-T_{\text {sat }}$, where $T_{\text {at }}$ is the saturation temperature. In a liquid in bulk, the appearance of the first bubble occurs because of homogenous nucleation, requiring substantial supersaturations. In a porous medium, however, the onset of phase change occurs by heterogeneous nucleation, as discussed below. We note that the pore wall curvature stabilizes interfaces, thus vapor-liquid equilibria are possible. Given that a bubble has formed (point $\mathrm{C}$ ), the pressure in the vapor phase at equilibrium 
is $P_{V}=P_{V O}\left(T_{L}\right)$. The corresponding supersaturation equals $\Delta P=P_{V}-P_{L}$. Superheat and pressure supersaturation are related to each other by the Clausius-Clapeyron equation. When the equilibrium superheat is exceeded, however, equilibrium can not be maintained and further growth takes place.

\subsection{NUCLEATION}

Nucleation processes in porous media are quite complex and there are still several questions that remain unanswered. However, certain progress has been made. It is apparent that, in almost all cases, we can eliminate homogenous nucleation as a plausible mechanism becauso of the high supersaturation requirements (of the order of several thousand psi, [105]), perfectly smooth and liquid wet solid surfaces and no trapped gases. All of these conditions are clearly unrealistic in the majority of cases, which do not involve explosive growth. It is rather evident, then, that the appropriate nucleation mechanism is that of heteregenous nucleation.

The latter has been analyzed by Yortsos and Parlar [105], who have proposed a nucleation model. This model assumes that there exist surface irregularities (cracks, scratches, pits), which are not liquid wet and they contain pre-existing or trapped vapor. For convenience in calculations, the nucleation sites were assumed of a conical shape. Furthermore, a self-similarity between host pore body and nucleation site sizes was postulated (Figure 3). Due to the assumption of preexisting vapor, the issue of (homogeneous) nucleation rate is bypassed. Therefore, the condition for (heterogeneous) nucleation is obtained simply by applying the Laplace equation when the vaporliquid meniscus in the cavity reaches the cavity mouth. As shown below, prior to this point the menisci are stable. Upon reaching the cavity mouth, the bubble becomes unstable, it rapidly grows to occupy the host pore body, and further expands to invade surrounding pores. With $W$ denoting the cavity or pit mouth radius, we have at the onset of nucleation of a site of size $W$

$$
P_{V}-P_{L}=\frac{2 \gamma}{W}
$$

This equation relates the cavity mouth radius $W$ with the supersaturation required for the nucleation of the cavity. To express this condition in terms of the host pore body radius, we adopt from Yortsos and Parlar [105] the geometric factor $\beta(\beta=W / r)$, which is the ratio of cavity mouth radius to pore body radius. Given the latter, and $\beta$, equation (3) provides the condition for a pore body of a given size to be occupied by vapor (nucleate). If $\beta$ is assumed constant, larger pores are 


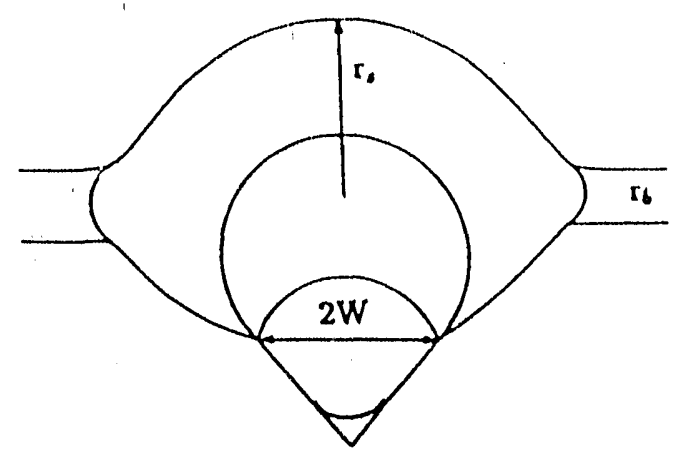

Figure 3: Schematic of a vapor bubble in a conical cavity.

more likely to nucleate first. We are certainly aware that this may not necessarily be the case and, in fact, it is more likely that $\beta$ is randomly distribited. However, such an assumption, while it considerably facilitates the calculations, it also does not detract qualitatively from the conclusions reached, particularly regarding the growth paitern. Of course, other expressions can also be used as desired (see Parlar and Yortsos [62]).

To examine in detail the nucleation process in a hydrophobic conical cavity, we consider the variation of the radius of curvature of the liquid-vapor interface with the volume of the vapor (Figure 4). A negative radius of curvature implies that the interface is concave to the liquid phase. As is well known (Parlar and Yortsos, [62]), stable interfaces correspond to configurations such that $d r / d v<0$, i.e. that the radius of curvature decreases with an increase in the volume of the vapor nuclei. From Figure 4 we can infer that, until the radius $r$ reaches $W$, the equilibrium state is stable, implying that the vapor remains confined in the cavity. However, after the meniscus reaches the cavity mouth, a further increase in the imposed supersaturation renders the equlibrium state unstable, and the bubble grows until its radius of curvature reaches the host pore body radius. Due to the pore wall constraints, further increase in the volume is associated with a decrease in the radius of curvature. This step is also stable, although it may not necessarily be an equilibrium 


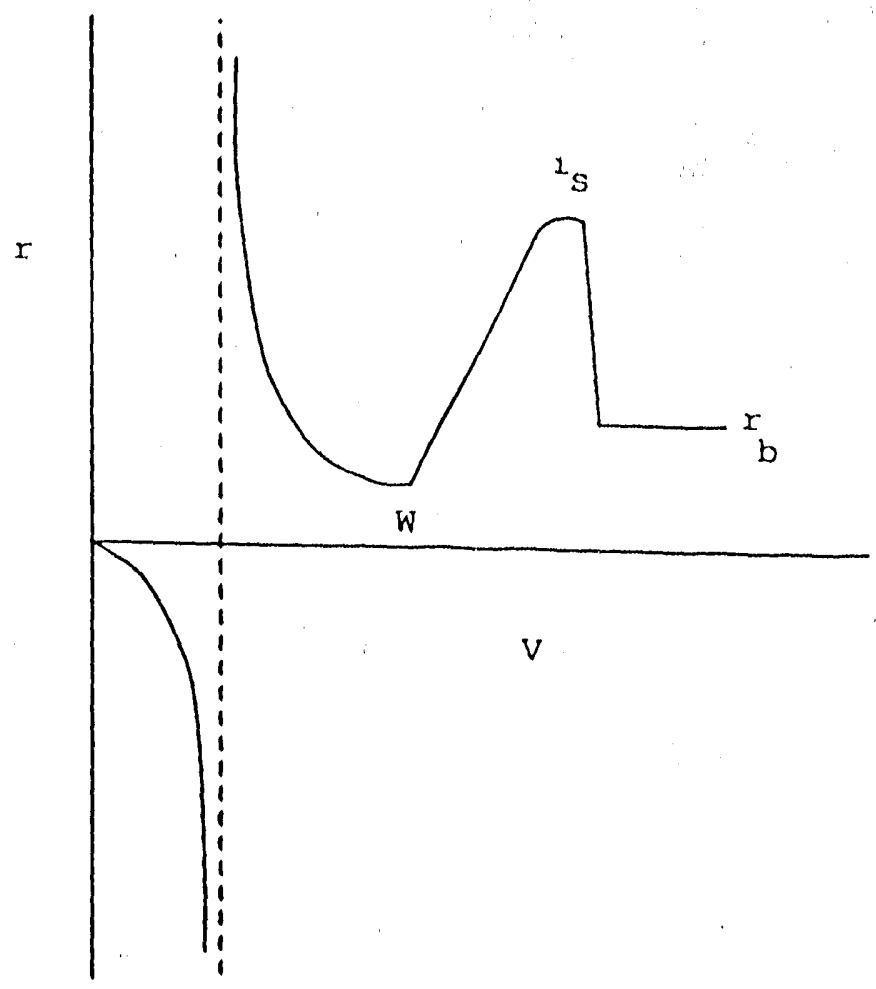

Figure 4: Schematic of vapor bubble radius-volume relation in a hydrophobic cavity.

configuration at the given supersaturation (its radius may be larger than $W$ ).

By contrast, a vapor bubble in an unbounded bulk liquid is in an unstable mechanical equilibrium state always, and it would either keep growing indefinitely or it would collapse. The fact that due to pore wall stabilization in a porous medium a vapor bubble can exist in stable equilibrium with a superheated liquid, is a major difference between the two cases.

In the above, it was assumed that no bubble detachment occurs due to gravity . A condition for the latter obtained by balancing surface and gravity forces, was cited in Yortsos and Parlar [105]. Using their estimate, we calculated that gravity-induced detachment will not take place in any pore with size less than $571.59 * \sqrt{\beta}$ micron, for the typical values listed in Table 1.

\subsection{BUBBLE GROWTH}

In this section, the process of bubble growth in a porous medium is studied. Having previously described phase equilibria and nucleation events, we may now proceed to formulate the bubble growth. This is controlled by surface, inertia, pressure and viscous forces, the driving parameter being the externally imposed superheat in the liquid. For simplicity, we again consider a single vapor bubble in an infinitely large porous medium occupied by a liquid (Figure 5). In a later 


$$
\begin{aligned}
& c_{p} \quad 1.059 \mathrm{Btu} / \mathrm{lbm} /{ }^{\circ} \mathrm{F} \\
& H_{0}^{\cdots+1}=15 \% \mathrm{~m} \\
& \text { k :.: Idarcy } \\
& l_{1} \ldots 841.75 \mathrm{Btu} / \mathrm{lbm} \\
& M 1=18(6) / m o l \\
& \text { H. } 202.819 \mathrm{psi} \\
& 7, \ldots 83^{\prime \prime} \mathrm{F} \\
& \alpha_{l}=6.7113 * 10^{-3} f t^{2} / h \\
& \gamma=60.0 \text { dynes } / \mathrm{cm}^{2} \\
& \lambda_{L}=0.386 \mathrm{Btu} / \mathrm{h} / \mathrm{ft} /{ }^{\mathrm{\omega}} \mathrm{F} \\
& \xi_{d}=1 \\
& \rho_{L}=54.31 \mathrm{ltm} / \mathrm{ft}^{3} \\
& \phi=0.35
\end{aligned}
$$

Table 1: Typical parameter values used.

section, we shall extend the model to consider growth from multiple bubbles.

In order to discuss the growth process in detail, the dimensionless parameters which control the growth regimes are, first, introduced. We follow Szekely and Martins [91] who studied the growth of a spherical gas bubble in a supersaturated bulk liquid. Values for the parameters used below are listed in Table 1. As in Yortsos and Parlar [106], the variable $\Phi$ expresses the importance of capillarity with respect to supersaturation

$$
\Phi=\frac{2 \gamma H_{o}}{\Delta P}
$$

where $H_{o}$ is the reference mean curvature of the menisci of the initial bubble. Depending upon the magnitude of $\Delta P$, growth is described by different regimes. For convenience, we may also express this paramater in terms of the imposed superheat $\Delta T$

$$
\Phi=\frac{\Delta T^{* *}}{\Delta T}
$$

where

$$
\Delta T^{* *}=2 \gamma H_{o} \frac{T_{L}^{2}}{\kappa P_{L}}
$$




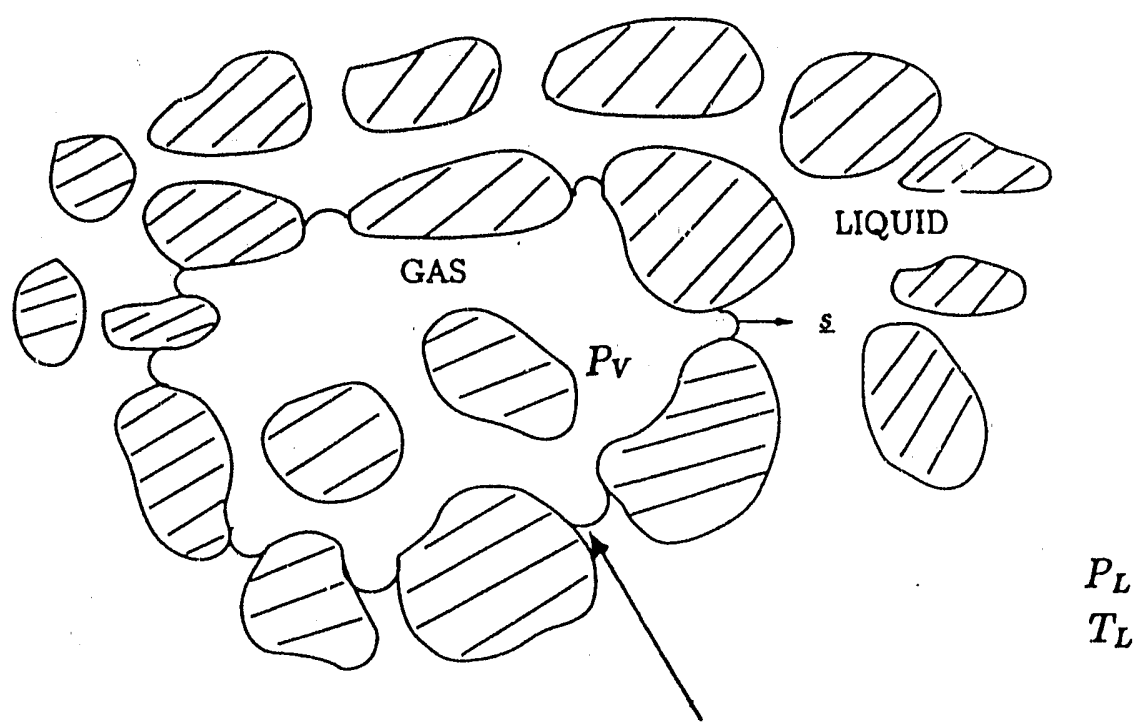

Growth by heat flux $\sim-k \nabla . T$

$$
\nabla^{2} T=0
$$

Figure 5: Schematic of single vapor bubble growth in porous medium.

and

$$
\kappa=\frac{L_{v} M}{R}
$$

Here, $\Sigma_{v}, M$ and $K$ are latent heat of vaporization, molecular weight and ideal gas constant, respectively. For the typical values listed in Table $1, \Delta T^{* *}=0.53^{\circ} F$. At low superheat values, $\Phi$ is $O(1)$ or less. Capillary-controlled growth occurs at $\Phi \sim O(1)$, in which case $\Delta P$ is of the order $2 \gamma H_{o}$. For the typical values in Table $1,2 \gamma H_{o}=1.157$ psi.

Alternatively, bubble growth can be expressed in terms of the Jacob number defined as

$$
J a=\frac{\rho_{L} c_{p} \Delta T}{\rho_{V} L_{v}}
$$

where $\rho_{L}, c_{p}$ and $\rho_{V}$ are liquid density, liquid specific heat capacity and vapor density, respectively. $J a$ is also another measure of the imposed superheat. By using the ideal gas law and after some manipulation, the Jacob number can also be expressed as

$$
J a=\frac{\Delta T}{\Delta T^{*}}
$$

where

$$
\Delta T^{*}=\frac{1}{\rho_{L} c_{p}} \frac{\kappa P_{L}}{T_{L}}
$$


For the typical values in Table $1, \Delta T^{*}=6.204^{\circ} \mathrm{F}$. Equivalently, $J a$ can be shown to be inversely proportional to the parameter $\Phi$

$$
J a=\frac{\Phi^{*}}{\Phi}
$$

where

$$
\Phi^{*}=\left(2 \gamma H_{o}\right)\left(\rho_{L} c_{p}\right) \frac{T_{L}^{3}}{\left(\kappa P_{L}\right)^{2}}
$$

For typical values, $\Phi^{*}=0.0408$. Important parameters which control the magnitudes of $J a$ and $\Phi$ are the liquid pressure and the temperature in the far field $\left(P_{L}, T_{L}\right)$. As $P_{L}$ increases, both $J a$ and $\Phi$ decrease. At moderate superheats, we also obtain from the Clausius-Clapeyron equation

$$
\Delta T=\frac{T_{L}^{2}}{\kappa P_{L}} \Delta P
$$

Finally, a measure of inertia forces is given by the growth rate parameter :

$$
B=\frac{J a^{2}}{\sqrt{G}}
$$

where

$$
G=\frac{R_{o}^{2}}{\rho_{L} \alpha_{L}^{2}} \Delta P
$$

and where $R_{o}$ and $\alpha_{L}$ are initial bubble radius and thermal diffurivity of liquid, respectively. The magnitude of $B$ is controlled by $J a$. Following Szekely and Martin [90], $B \ll 1$ implies heat transfercontrolled growth, while $B \gg 1$ implies inertia-controlled growth. In our subsequent applications, $J a \ll 1$, thus the growth process is heat transfer-controlled only and inertia forces are, therefore, negligible. The opposite is, of course, valid in explosive growth.

In the following, we shall examine regimes for vapor bubble growth in the porous medium for a range of superheat values.

\subsubsection{Low Superheats}

When the imposed superheat is low, the growth process is heat transfer-controlled and inertia and viscous forces are negligible, as discussed above. We demonstrate this by expressing the energy balance in the liquid-occupied space in the dimensionless form

$$
J a\left(\frac{\partial \theta}{\partial \tau}+\underline{\nu_{L}} \cdot \nabla \theta\right)=\nabla^{2} \theta
$$


where

$$
\begin{gathered}
\theta=\frac{T_{L}-T_{s a t}}{\Delta T}, \\
\tau=\frac{\alpha_{L} t J a}{R_{o}^{2}} \\
\underline{\xi}=\frac{\underline{x}}{R_{o}}
\end{gathered}
$$

and

$$
\underline{\nu_{L}}=\frac{R_{o} \underline{u_{L}}}{\alpha_{L} J a}
$$

In the above, $\underline{u_{L}}, \underline{x}$ and $t$ are liquid velocity, spatial distance and time, respectively. The energy balance at the vapor-liquid interface completes the problem

$$
\nu_{s}=\frac{\partial \theta}{\partial s}
$$

At the vapor-liquid interface, the Laplace equation is also satisfied. At low superheats, inertia and viscous forces are negligible and the momentum balance simply reads

$$
\Pi=\Phi \eta
$$

where

$$
\Pi=\frac{P_{V}-P_{L}}{\Delta P}
$$

and

$$
\eta=\frac{H}{H_{o}}
$$

where $H$ is the mean curvature. Alternatively, $\Pi$ at the interface can be related to $\theta$ by the Clausius-Clapeyron equation. The boundary and initial conditions are

$$
\theta=1 \quad \text { at } \quad \underline{\xi} \rightarrow \infty \quad \text { or } \quad \tau=0
$$

and

$$
\underline{\nu_{L}}=0 \quad \text { at } \quad \underline{\xi} \rightarrow \infty
$$

The energy balance in the solid body obeys the heat equation. In dimensionless form we have

$$
J a \frac{\partial \Theta}{\partial \tau}=\psi \nabla^{2} \Theta
$$


where

$$
\Theta=\frac{T_{0}-T_{\text {sat }}}{\Delta T}
$$

and $T$, and $\psi$ are the solid temp rature and the ratio of solid to liquid thermal diffusivities, respectively. Also, at the solid-liquid interface, a thermal energy balance yields

$$
\frac{\partial \theta}{\partial s}=\mu \frac{\partial \Theta}{\partial s}
$$

where $\mu$ is the ratio of solid to liquid conductivities. At low superheats, $J a \ll 1$, both balance equations above reduce to the Laplace equation, provided also that $J a \ll \psi$,

$$
\begin{aligned}
\nabla^{2} \theta & =0 \\
\nabla^{2} \Theta & =0
\end{aligned}
$$

Therefore, under such conditions the temperature fields in both liquid and solid are quasi-static. In principle, these equations can be solved with the given boundary conditions. On the other hand, a simpler analysis is possible depending on the magnitude of $\Phi$.

(a) When $\Phi \sim O(1)$, capillary forces solely control the growth and the process is of the percolation type, a process which has been well studied (Yortsos and Parlar [106]). The rules for the occupancy of pores by the vapor phase given in [106] can be used with minor modifications. Since percolation (ordinary or invasion) growth patterns exist only in quasi-static fields, the above are applicable only if the temperature increase rate is not too large. Yortsos and Parlar [106] developed a condition for the case of pressure depletion in solution gas-drive systems. Here, we shall modify their result for the case of boiling in porous media. We obtain

$$
Q \ll \frac{\Delta T^{*}}{\tau^{*}} J a^{3}
$$

where

$$
\tau^{*}=\frac{\xi_{d}^{2} k}{\phi \alpha_{L}}
$$

and $\xi_{d}, k$ and $\phi$ are the dimensionless correlation length in the percolation process, permeability and porosity, respectively. Taking $J a \sim 10^{-3}$ and substituting $\Delta T^{*}=6.204^{\circ} \mathrm{F}$ in the condition above, gives $Q \ll 32.497^{\circ} \mathrm{F} /$ day for the percolation growth pattern to be valid.

(b) When $\Phi \ll 1$, capillarity is not important, and the process is of the DLA (DiffusionLimited Aggregation) type, in the absence of solid conduction. In this case, equilibrium states are 
not possible and there is continous growth until full occupancy of the porous medium by vapor. Clearly, at least for this case, classical concepts such as relative permeabilities are not valid.

\subsubsection{Moderate and Large Superheats}

When the superheat is moderately large, $J a$ is $O(1)$. Under the condition $B \ll 1$, inertia and viscous forces are still negligible. Therefore, the growth process is heat transfer-controlled, except that now the temperature field is no longer quasi-static. This problem is quiie complex, requiring for its solution the consideration of both convection and unsteady state terms. Finally, at large superheat, $J a \gg 1$, while also $B \gg 1$, implying that inertia controlls growth. This is the case of sudden (explosive) growth. This case falls outside our scope.

All equations developed above also apply for multiple bubble growth. Growth from many nuc ation sites was studied by Yortsos and Parlar [105] who applied their percolation model at low s tpersaturation in infinite systems. We have modified their model to the boiling case. Results of a simulation in a square lattice are shown below. The original model was developed for infinite systems, where there is no depletion of supersaturation. To account for this, we further modified our numerical model by considering the adiabatic case, where the available energy is depleted by the conversion of liquid to vapor. A final modification involves the solid participation to heat transfer. In general, three different regimes can be identified according to the ratio of conductivities $\left(\mu=\lambda_{s} / \lambda_{L}\right)$ :

$\mu \ll 1 \rightarrow$ where only the liquid is conducting

$\mu \sim 1 \rightarrow$ where both solid and liquid participate in the conduction, and

$\mu \gg 1 \rightarrow$ where only the solid is conducting

Each of these three cases finds many applications. The model in [105] corresponds to the first. limit, where the solid conductivity is small and its participation to heat transfer is negligible. On the other hand, typical boiling applications in porous media lie between the second and the third cases, with the ratio $\mu$ as high as 10 .

The three modification are discussed below under the general heading of percolation models. Ahhough they share common aspects, the processes are different. The first case is slow, uniform heating, the second corresponds to adiabatic expansion, although at uniform temperature and pressure, while the third application involves static vapor-liquid distributions in the presence of a 
temperature gradient. Two variations of the latter are considered.

\subsection{PERCOLATION MODELS}

As noted previously, at a low superheat and for $\Phi \sim 1$, the growth process is of the percolation type. In this case, infinitesimally small $\Delta T$ steps corresponding to a slow heating rate are imposed, such that the supersaturation is always balanced by capillary pressure. The percolation model presented below differs from that of Yortsos and Parlar [105] in that the network is a square lattice and the effort here is mainly numerical. We use both bond and sites distributions. The rules for the activation of the nucleation sites and the occupancy of pore space by the vapor are simple. Given a superheat $\Delta T=T_{L}-T_{\text {sat }}$, pores with radius

$$
r \geq R^{*}=\frac{2 \gamma T_{s a t}}{\rho_{V} L_{v} \Delta T}
$$

are occupied by the vapor phase if they are already connected to vapor-occupied sites or to nucleation sites. New nucleation sites are activated if the host pore bodies have a large enough radius

$$
r_{s} \geq \frac{R^{*}}{\beta}
$$

As pointed out above, a major difference between this model and classical ordinary percolation model is nucleation. Obviously, the classical ordinary percolation model is recovered in the limit $\beta=1$.

\subsubsection{Ordinary Percolation}

Simulation results are shown in Figure 6, where three different stages of growth are represented. For this example, a square $(30 \times 30)$ lattice network is used. Pore body and throat size distributions were taken from a uniform distribution. In Figure 6, gray denotes vapor-occupied sites, black denotes nucleation sites and white denotes liquid-occupied sites. As the supersaturation increases, more nucleation sites are activated. The predictions for this model are similar to those in the analytic study of Yortsos and Parlar [105]. A critical supersaturation exists for the onset of bulk vapor flow, and relative permeabilities can be defined as a function of the nucleation parameter $\beta$. Figure 7 shows schamatics of the latter as obtained by the study in [105]. We refer the reader to their paper for further details. 

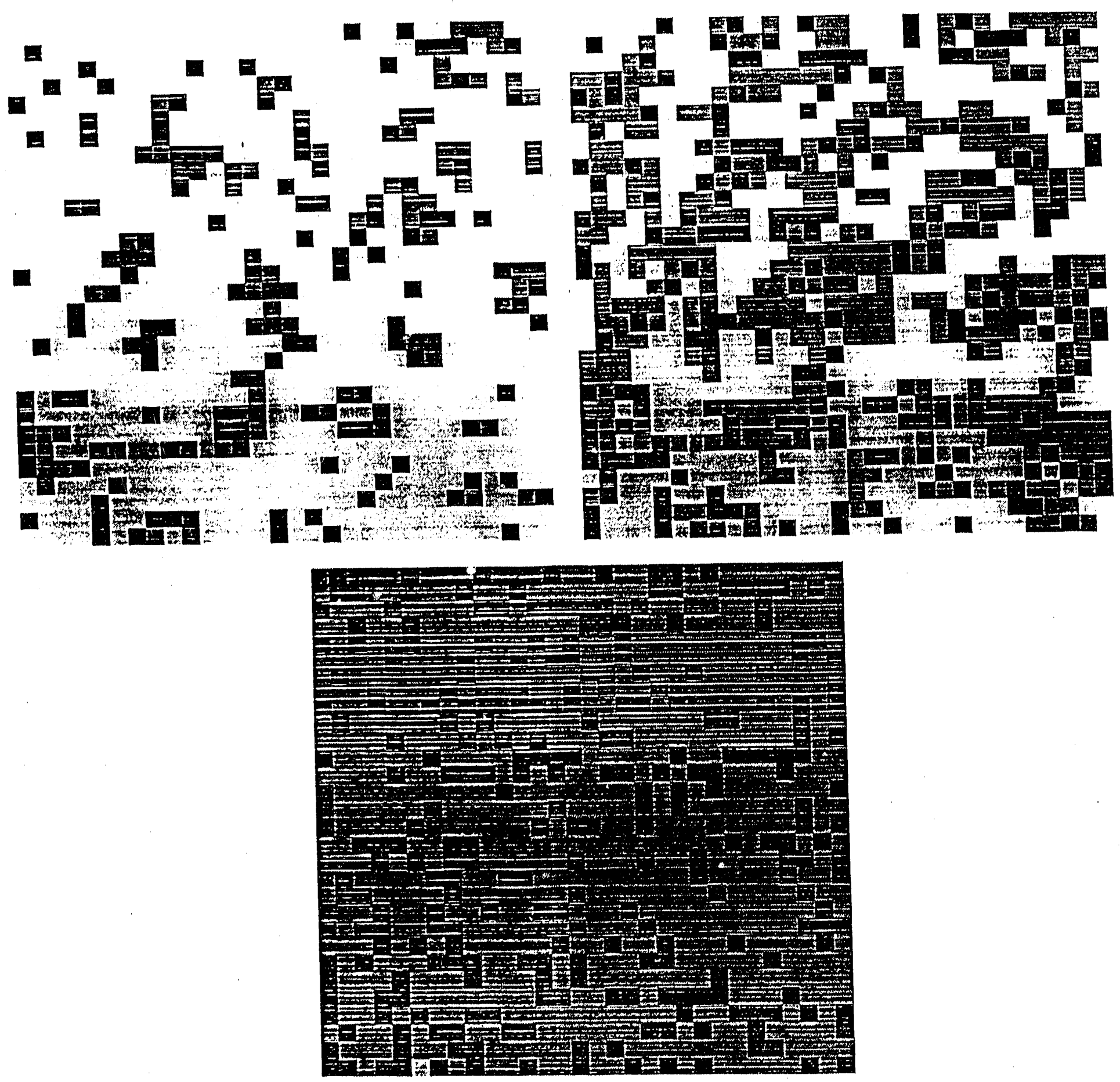

Figure 6: Three different stages of vapor growth in the pressure depletion case. 


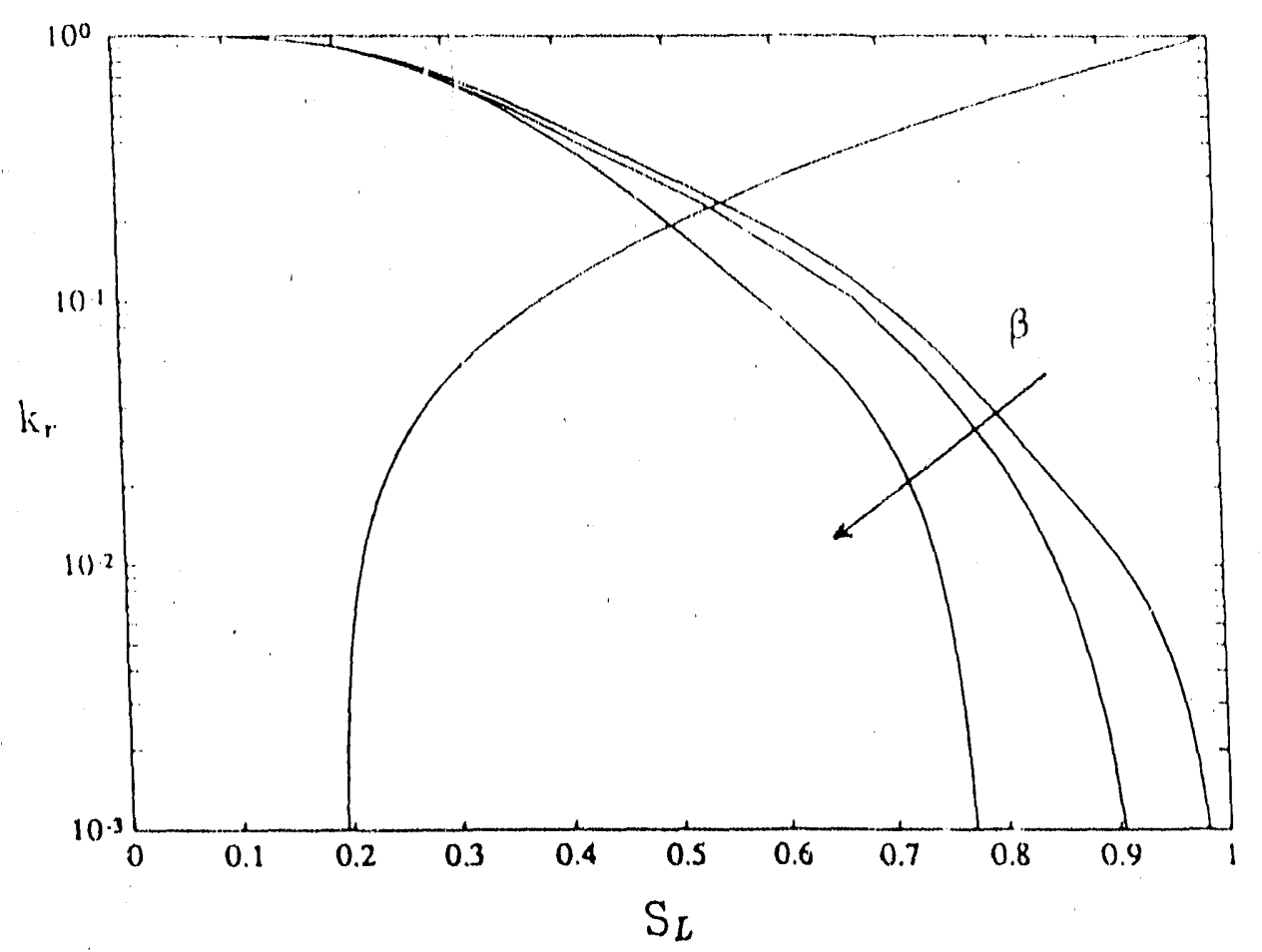

Figure 7: Schematics of relative permeabilities as a function of $\beta$.

Conditions for the validity of this model were developed above. As pointed out, this process is a slow, spatially uniform heating of the liquid in the porous medium. Clearly, this is only a first approximation to the actual process. One possible modification regards the depletion of superheat. To model the latter, we have simulated vapor growth by pressure reduction in an adiabatic system. Since vapor expands and displaces liquid, the available amount of initial entalphy is constantly reduced. Therefore, further growth can only occur by pressure depletion. Results are shown in Figure 8. At the end of the process the pressure is reduced from 202.8 psi to 185 psi, while the corresponding temperature reduction is somewhat less, from $383^{\circ} \mathrm{F}$ to $375.9^{\circ} \mathrm{F}$ for the parameters of Table 1. Although the amount of imposed supersaturation or superheat changes, the growth pattern still remains the same. One concludes that consideration of finite volume and adiabatic ronditions does not change qualitatively the previous results.

'The next modification involves processes where the heat transfer in both liquid and solid must be considered. To solve this problem, the energy equations in both liquid and solid must be coupled. It. was previously shown that at conditions of low $J a$, these equations satisfy the Laplace equation. For their solution, we used both iterative SOR (Successive Overrelaxation) and direct (inverting malrix.) methods. The SOR iterative method was preferable, because it required much less memory, 

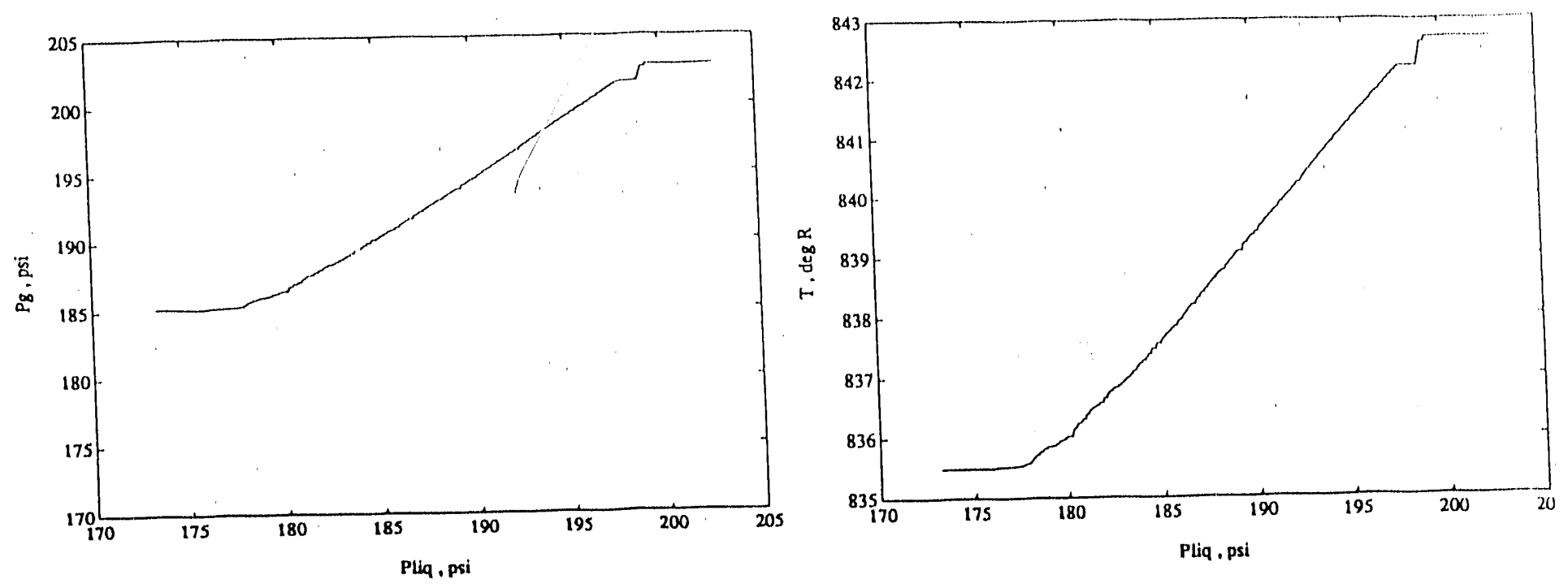

Figure 8: Finite volume effect on the depletion of supersaturation and superheat imposed.

although its computation time requirements were higher. In the simulations, periodic or no-flow boundary conditions were used at the sides, and constant temperature boundary conditions were used at the ends. Such simulations represent the steady-state solution of a vapor-liquid system across which a temperature difference is imposed. In our approach, we make use of the previous rules for vapor occupancy of a site, including nucleation. Note, however, that countercurrent flow was not allowed. A temperature gradient in the vapor phase necessarily implies a corresponding pressure gradient, thus vapor flow. At steady state, this must be counterbalanced by liquid flow in the opposite direction. By neglecting such effects we have imposed a significant restriction, the effects of which are currently investigated.

For the nucleation of a pore, we used the parameter $\beta$ which assumes that greater size pores are more likely to nucleate. Alternative distributions for nucleation were also used. We first examined the effect of the magnitude of superheat $(\Delta T)$, which is the difference in temperatures at the two ends. Two different values $\left(0.01{ }^{\circ} \mathrm{F}\right.$ and $\left.2^{\circ} \mathrm{F}\right)$ were used to illustrate rate effects on growth (Figure 9). Clearly, this is an illustration of the competition between nucleation and growth. In the specific example, $\boldsymbol{\mu}=\mathbf{0 . 0 0 1}$, thus all heat is transferred through the liquid only. For small $\Delta T$. steps, the final pattern reached corresponds to a smaller steam zone. This is because once all sites 

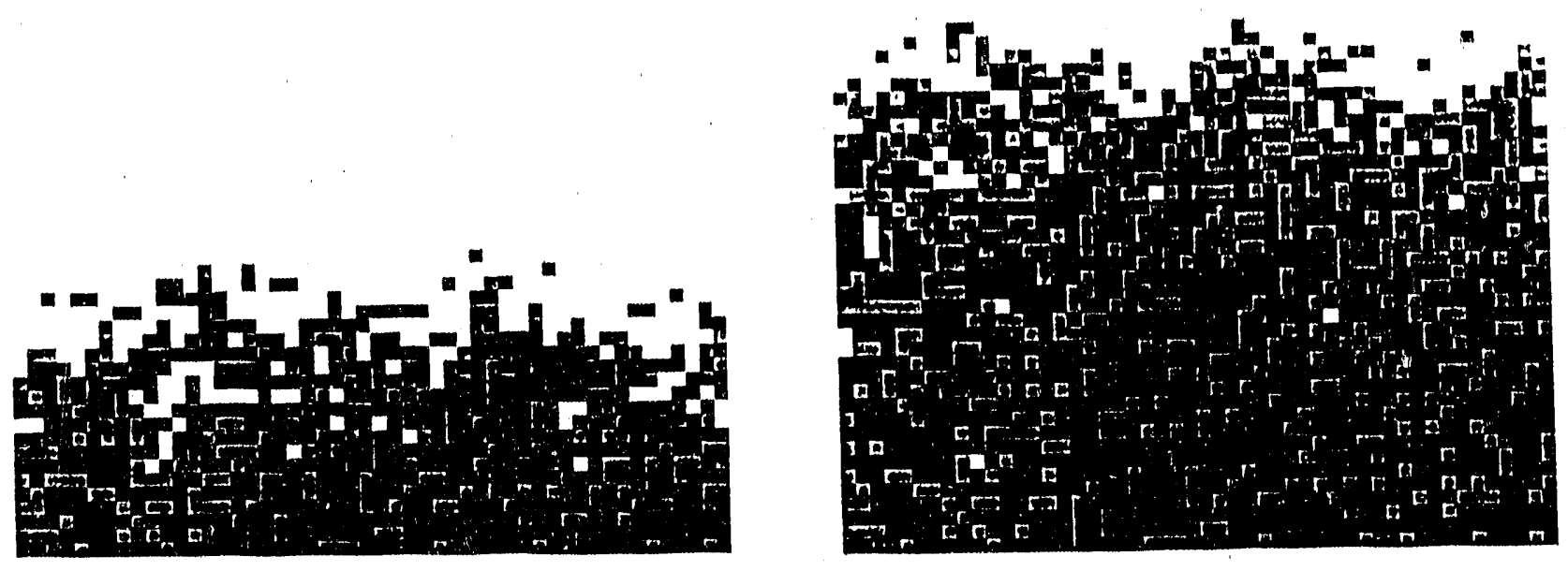

Figure 9: The final growth pattern for two different values of $\mu$.

at the entrance are occupied, heat transfer occurs through the vapor phase only, the conductivity of which is much less than that of the liquid. Reaching in one step the same temperature value at the entrance (as in the case of large step, Figure $9 \mathrm{~b}$ ) results in better heat transfer and higher occupation of pores by vapor. The effect of $\mu$ is shown in Figure 10 for two simulations, one corresponding to $\mu=0.001$ and the other to $\mu=10.0$. The increase in the solid conductivity results in a significant increase in the vapor occupation.

\subsubsection{Gradient Percolation}

An alternative method for describing the above problem is by gradient percolation. This concept has been. studied extensively in recent years as a variation of classical percolation. It is recalled that in ordinary percolation theory, the probability for the occupation of a site is uniform in space. In the case of gradient percolation, on the other hand, the probability density is not constant, but it is given by a function specified a priori, which is variable in space. This difference substantially changes the pattern of growth from that of classical percolation.

Rosso et al. [79] were the first to introduce gradient percolation in their study of diffusion in solids. They took the complementary error function to describe the probability distribution in 

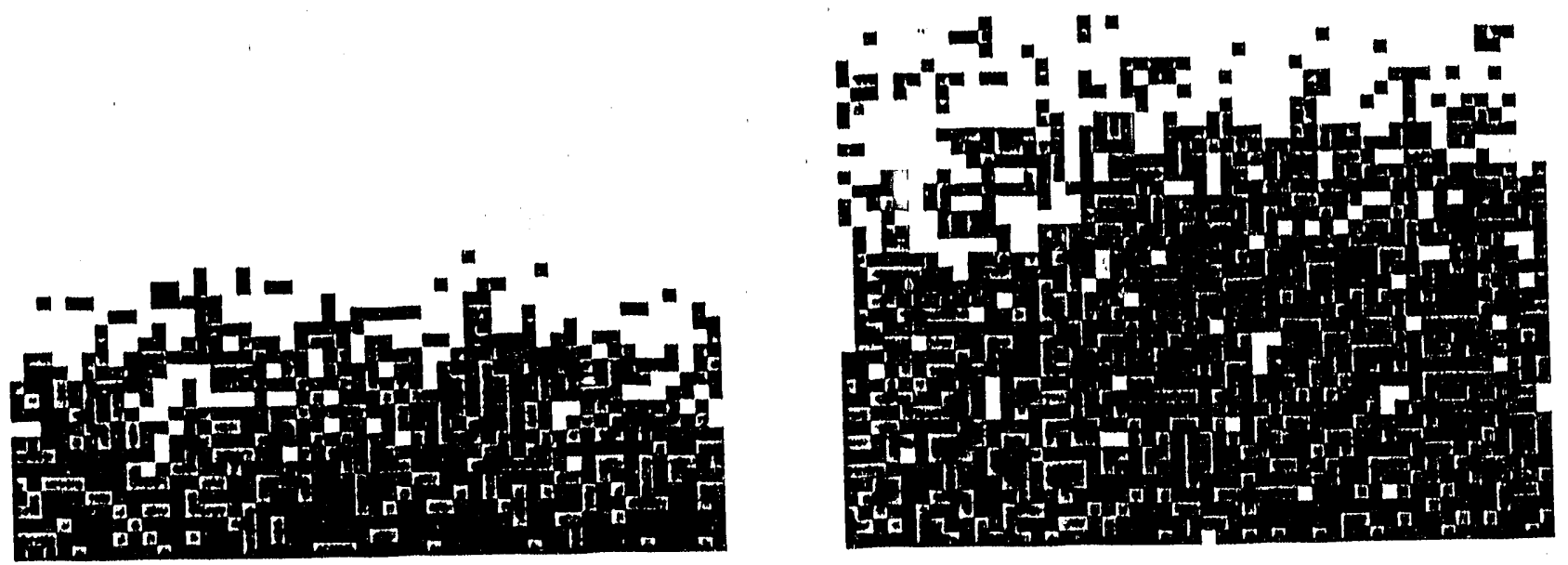

Figure 10: The final growth patterns for two different values of $\mu$.

space, and applied the method to two-dimensional, square and triangular lattice networks. Using certain clustering and occupation rules, they examined the nature of the diffusion front created. They found out that this front is fractal (with dimension 1.75), while the probability value at the mean position of this front is related to the percolation threshold of ordinary percolation, $p_{c}$, corresponding to a two-dimensional network. Gouyet et al. [72] calculated many properties of gradient percolation using a linear profile. In a subsequent study, Gouyet et al. [73] extended their previous 2-d models to a 3-d cubic lattice. They observed significant differences between 2-d and 3-d simulations. In particular, the shape of the density profile at the frontier changed. In the $3-d$ case, the density profile is not Gaussian-like, but it extends over a finite concentration region. Furthermore, the probability density value at the mean frontier position was not close to $p_{c}$. Rosso et al. [31] used the previous model to study scaling properties of the front. They found that this front is also a fractal object with dimension $D=2.5$.

Concerning bubble growth in porous media, the application of gradient percolation is appealing. However, since the temperature gradient is fixed a priori and it is not coupled to the phase distribution, this process corresponds to the case where solid conduction predominates, $\mu \gg 1$. At such conditions, the temperature field is decoupled from the occupancy scheme, which it actually dic- 
tates. Wo note, again, that this scheme does not include fluid flow considerations. For appllention to boiling in porous media, we modified gradient percolation by adding bond statistics, nucleation effects and a fixed temperature gradient. The probability density functic: for site occupancy here also depends on the pore size distribution. We considered uniform size distributions for sites and bonds, and a linear temperature distribution. Growth patterns for both ordinary and modified gradient percolation models are shown in Figure 11. Dark colored squares are vapor-occupled sites. As see- in the figures, the two patterns are quite d:fferent as expected. A steam front is clearly distinguishable in the gradient percolation case. The parameters affecting the occupancy are pore and bond size distributions, and the values of $\beta$ and $\Delta T$. As $\beta$ increases, more nucleation sites exist and this results in higher occupation of pores by vapor (Figure 12). In the figures, gray denotes vapor-occupied sites, while black denotes the interface which separates the infinite clusters of liquid-occupied sites and vapor-occupied sites. An increase in $\beta$ shifts this interface toward the liquuid sicle.

\subsection{CONCLUSIONS}

In this chapter we applied the general methodology developed by Yortsos and Parlar [105], in their study of solution gas-drive processes, to the case of boiling (liquid-to-vapor) phase change in porous media. Nucleation, pore microstructure and process paraneters were considered. The case of uniform heating of the medium at low heating rates was described by a percolation process. The constraint that the heating rate must be significantly lower than $32.49^{\circ} \mathrm{F} /$ day was derived. For certain cases relevant to steam injection this may be a tight constraint. However, if such conditions prevail classical, relative permeability functions and critical saturation values can be employed as discussed. 'To account for temperature gradients during the growth process, we have considered two modifications of the above model, one of which is akin to gradient percolation. In the second case, conduction in the solid dominates the heat transfer, while the former accounts for heat transfer in beth the liquid and the solid, but in the absence of flow. Although an improvement over the uniform percolation model, these modifications still have a serious internal inconsistency, namely thry neglect flud flow. We are currently involved in an effort to resolve the latter. 


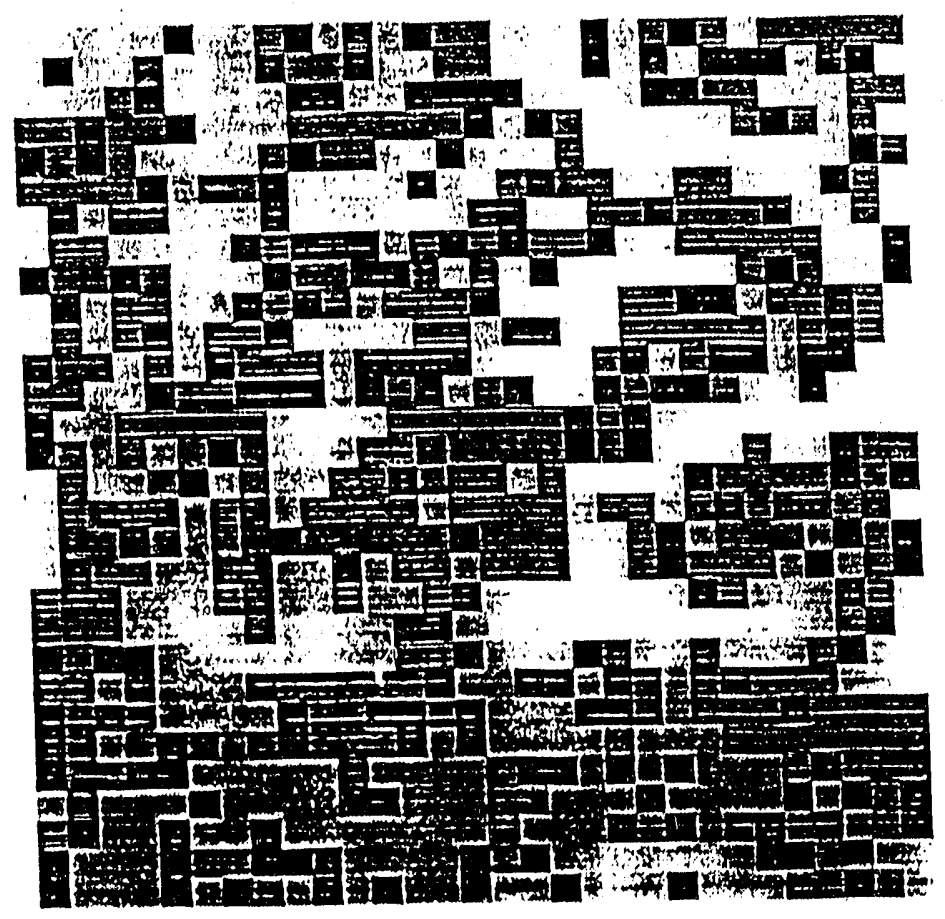

(a)

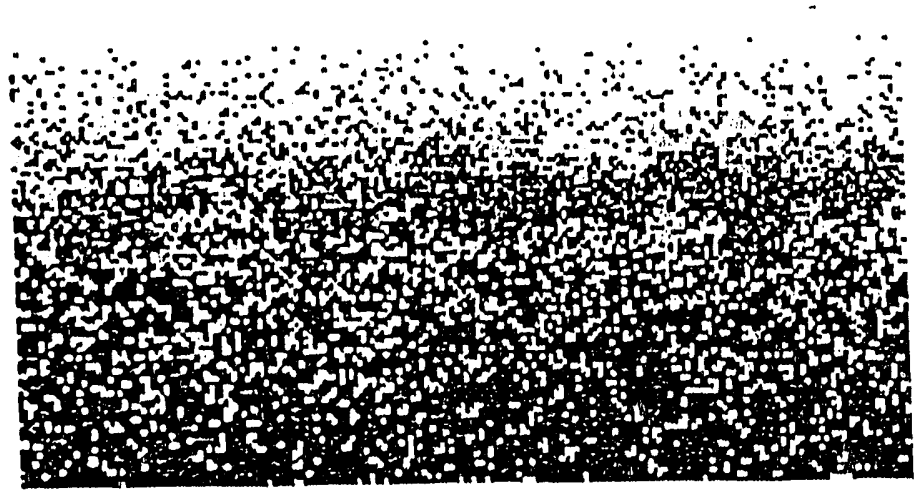

(b)

Figure 11: Ordinary (a) and gradient (b) percolation growth patterns. 


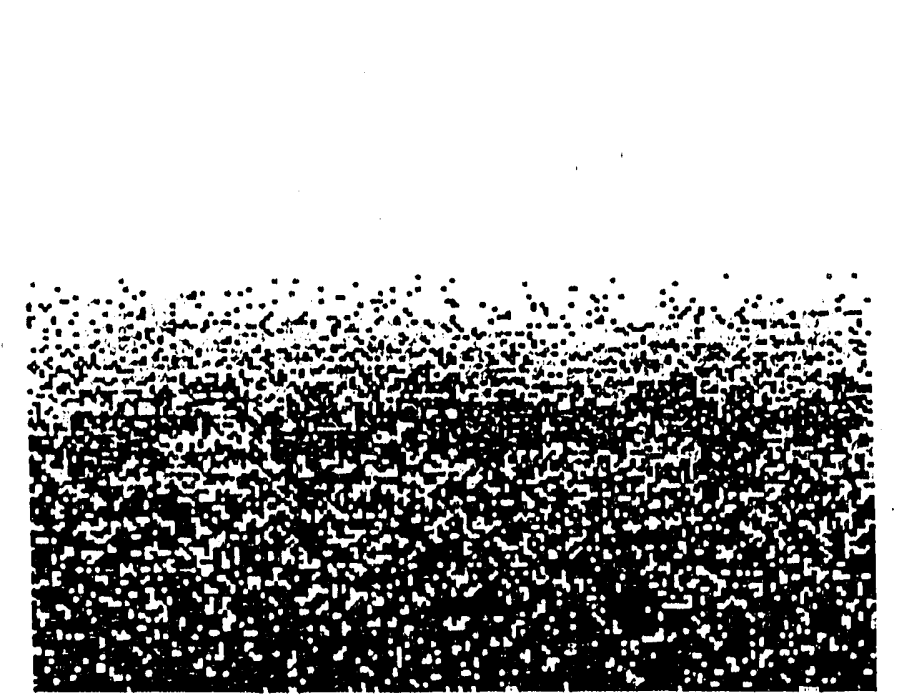

(a)

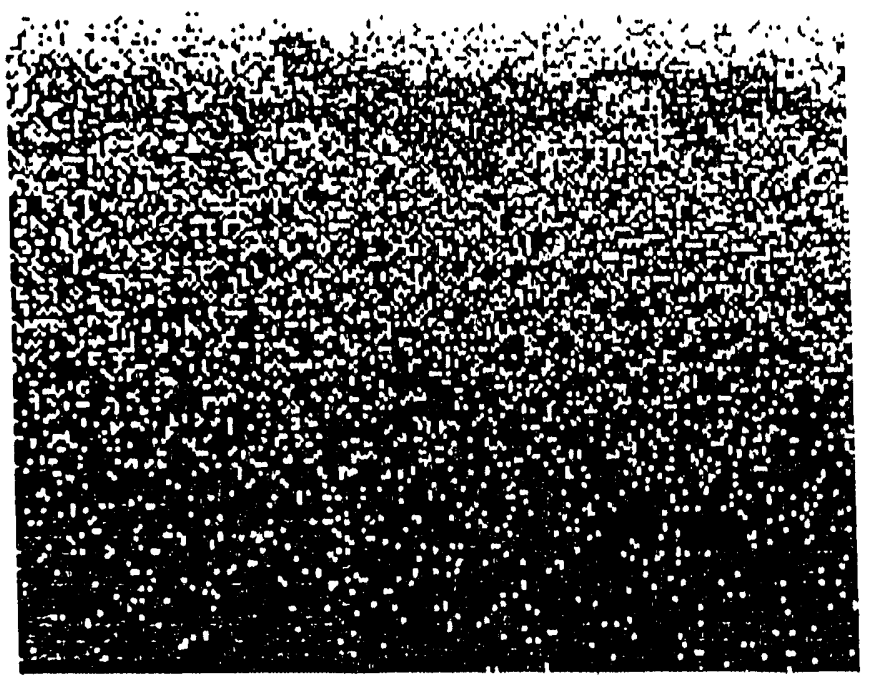

(b)

Figure 12: The modified gradient percolation growth patterns at two values of $\beta$. 


\title{
3 STEAM INJECTION VISUALIZATION EXPERIMENTS IN HELE-SHAW CELLS AND GLASS MICROMODELS
}

\author{
X. Kong, M. Haghighi, R. Prasad and Y.C. Yortsos
}

\subsection{INTRODUCTION}

Flow visualization experiments have been successfully employed in reservoir engineering research for many years $[15,39,94,102]$. The techniques used greatly enhance our understanding of flow phenomena. This is particularly necessary in displacement processes, where an accurate description of the dynamics of the interface is paramount to process modeling. Typical visualization experiments involve 2-D geometries in transparent Hele-Shaw cells and glass micromodels. Although such geometries lack important 3-D aspects, they are nevertheless valuable for the insight they offer on the micromechanics of the displacement.

Much work has been done on the visualization of immiscible flows (drainage or imbibition). Experiments in glass micromodels have also been reported in solution gas-drive processes and in foam generation and propagation $[3,16,21,58]$. Surprisingly, visualization of steamfloods, which constitute a major part of current EOR methods, has not been attempted to date (although a preliminary investigation was conducted in our lab several years ago). Reasons for the lack of success in this direction include the difficulties involved in the control of heat transfer and the diminished strength of glass at higher temperatures and pressures.

Although these technical problems are difficult to overcome, visualization studies are very valuable, even in the absence of heat transfer control. For example, while much work has been done on steamfloods in laboratory cores, often with the use of partly scaled experiments $[24,84,87]$, the results obtained are typically of the input-output type, and they cannot provide insight on the interactions at the pore-level. An understanding of the latter is very important for the validation of theories, for the refinement and improvement of models, for instance for relative permeabilities and capillary pressure, and for process simulation.

For this reason, we have underta.ken an experimental study with main objective the visualization of steam inejction processes in Hele-Shaw cells and in glass micromodels. The first study is expected to shed light on oil displacement by steam in planar geometries. To some degree, the geometry parallels thist of a smooth fracture, hence a direct application to fractured systems is possible. 


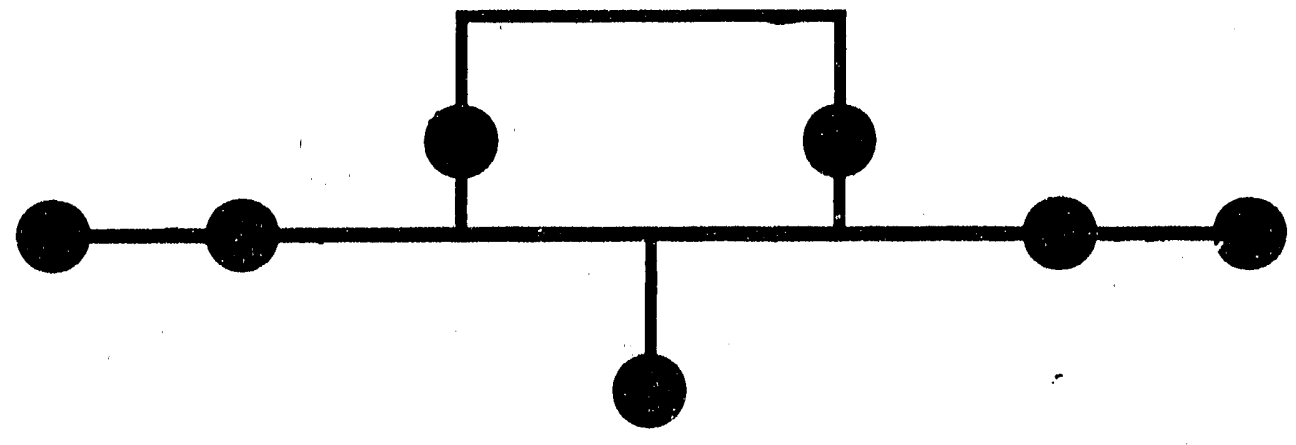

Figure 13: Glass micromodel with dead ends.

At the same time, it should be recalled that flow in Hele-Shaw cells at low Reynolds numbers is potential and can be approximated as Darcy flow, although in the absence of noise. Since the latter is invariably present in actual porous media due to microstructure, the analogy between Hele-Shaw and porous media displacement is somewhat tenuous. To partially remedy this, we have also conducted a study in glass micromodels, which allow for effects of pore structure to be investigated.

\subsection{EXPERIMENTAL}

Two model geometries, Hele-Shaw cells and glass micromodels, both constructed from pyrex glass, were used for steam displacement. The cell was of dimensions $24.5 \mathrm{in}$. $x$ 12in. $x 0.035 \mathrm{in}$. and consisted of two glass plates of $1 / 2$ in. thickness. Slightly modified etching methods (longer exposure to acid etching, faster fusion) were used for the construction of the pyrex micromodels. One pattern for the micromodel was taken from Mohanty [55], and was selected such that dead-end effects and effects of bypassing could be studied (Figure 13). Another pattern was based on a fractal network created by the techniques of Chapter 5 .

Two types of oil were used, a synthetic oil Dutrex 739, taken as represcintative of heavy oil, and 


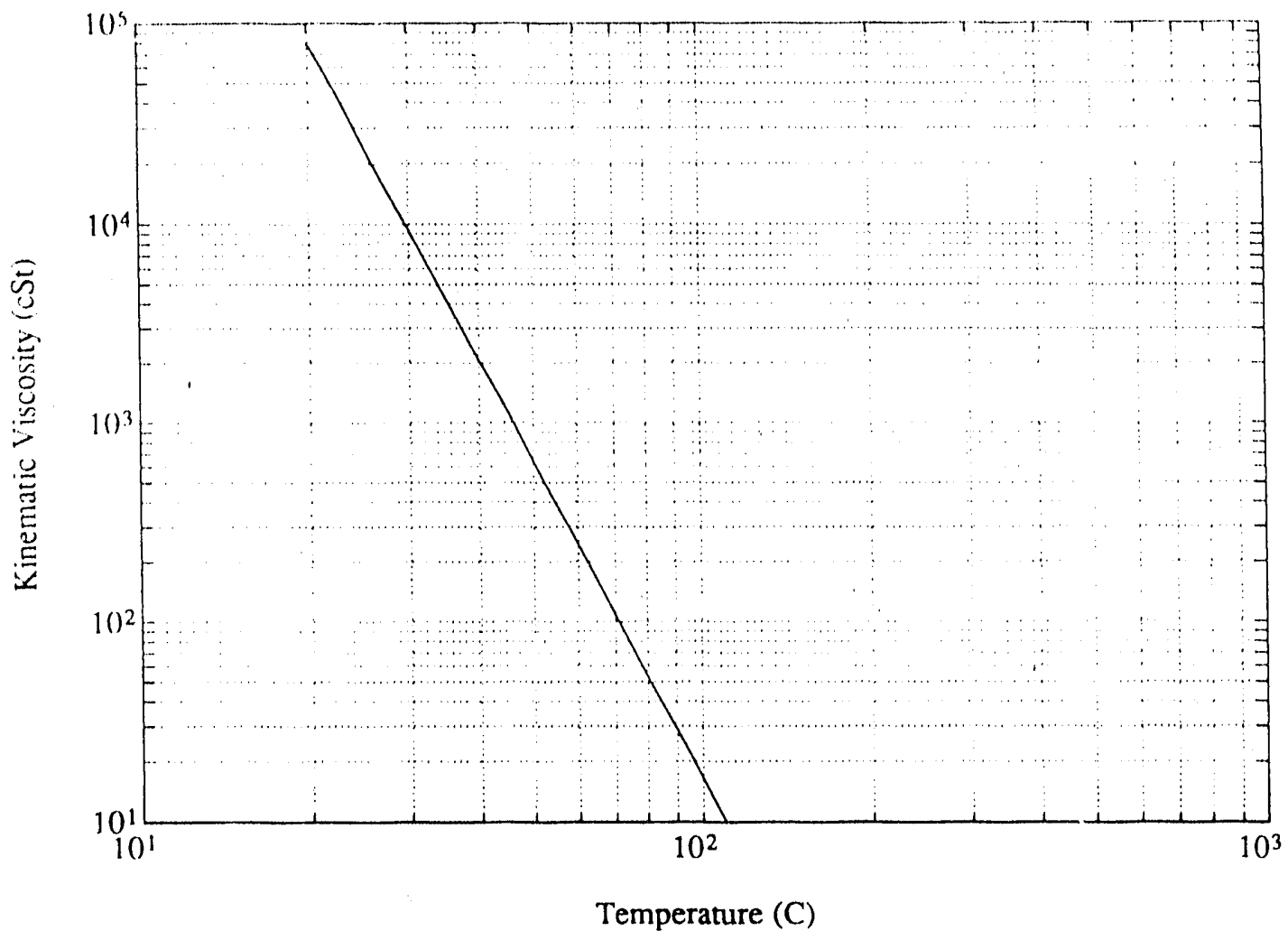

Figure 14: Temperature-shear viscosity relation for Dutrex 739.

mineral oil, as a representative of light oil. The temperature-shear viscosity relation for Dutrex 739 is plotted in Figure 14. It is evident that viscosity reduction is significant for this fluid. As became apparent from our study, significant non-Newtonian effects were also observed during its displacement. Non-Newtonian rheology, although complicating the interpretation of experiments, is certainly a prominent feature of most heavy oils. The mineral oil used was Newtonian, with room temperature viscosity of $189 \mathrm{cp}$.

Variable water injection rates and temperatures were used, typically in the range $0.1-3 \mathrm{cc} / \mathrm{min}$. Temperature control was not attempted at this stage. Instead, a known power source was applied to the steam generator, which was insulated, but not perfectly adiabatic. Injection temperatures fluctuated to within a few ${ }^{\circ} F$ around a mean value. In most of the runs involving steam, the injected steam was superheated. Neither the Hele-Shaw cell nor the glass micromodel were insulated or otherwise equipped with heat loss control, which simply occureri by heat conduction through the glass to the surroundings. This is certainly an important problem, that may affect the quantitative, although not the qualitative, analysis of our results. A separate, hut equally import.ınt, proliem regards the low injection temperature (pressure) typically used in order to prevent fracturing of the glass. We are currently experimenting with ways to resolve both these problems. A total of 15 
runs were conducted so far. All experiments were run with the cells in the horizontal position.

\subsection{RESULTS AND DISCUSSION}

We shall discuss in the following two main subjects: (i) Mechanisms of displacement in Hele-Shaw cells; (ii) Effects of pore structure as inferred from displacement in glass micromodels.

\subsubsection{Mechanisms of Displacement in Hele-Shaw Cells}

We consider as our base case the displacement of Dutrex 739 by steam at the rate of $3 \mathrm{cc} / \mathrm{min}$ and injection temperature $239^{\circ} \mathrm{F}$. In the subsequent pictures, taken from a still videotape, displacement is from left to right. One injection and one production ports are used. In the colored pictures, red is the color of the original oil, and bright white is steam. Condensed water flows typically between residual oil films attached to the two glass plates and left behind the water front. Their color is yellowish.

Figure 15 shows the initial part of the displacement. Due to the rapid heat losses and the contact with initially cold oil, steam condenses rapidly and the original displacement of oil is by the condensed water only. This displacement is unstable and produces viscous fingers similar to the case of isothermal displacement, although of a different intensity and pattern. Nevertheless, repeated tip splitting is observed, as is common in very unstable displacement [38]. The pattern laid by the condensed water is important, because it substantially influences the paths to be followed by the injected steam. After some period of injection, a steam zone is established near the injection port (Figure 16). We can visually separate vapor from liquid because we can identify clearly their interface and also because of their different colors. We recall that the liquid is covered on both sides by yellow, residual oil films that coat the glass plates. On the other hand, upon contact with vapor, the plates become very clean, perhaps by a steam distillation mechanism. A close up of this is shown in Figure 17. One can distinguish traces of what appear to be oil components. We have not undertaken a more detailed study and have deferred this to the future. Eventually no residual films are left behind. This was consistently observed in all runs.

Perhaps the mosi interesting features of these experiments are the shape and properties of the steam front. The following were commonly observed: The front was never stationary, or even quasistationary. Instead, it fluctuated constantly, and its advancement was unlike that of immiscible 


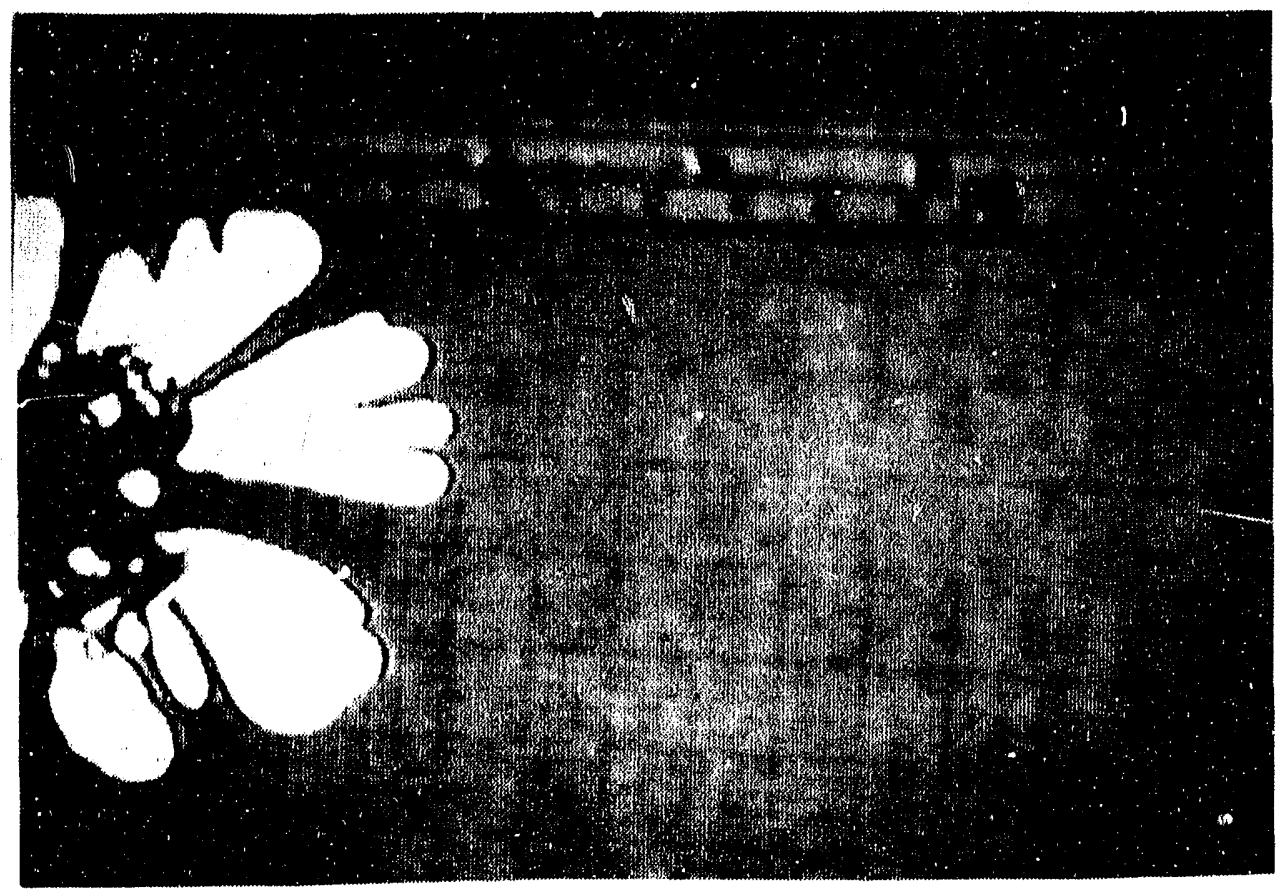

Figure 15: Initial stage of displacement.

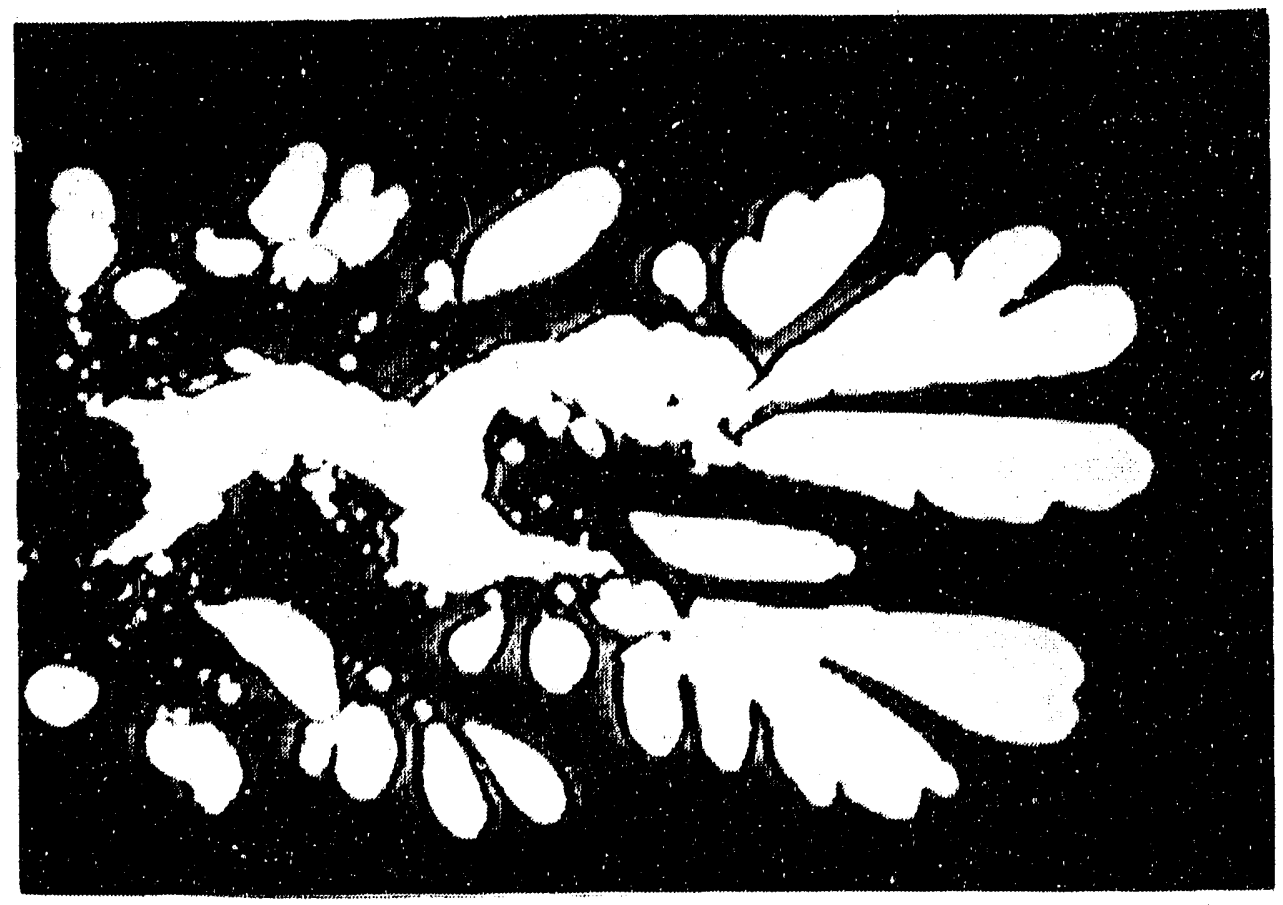

Figure 16: Initial steam zone. 


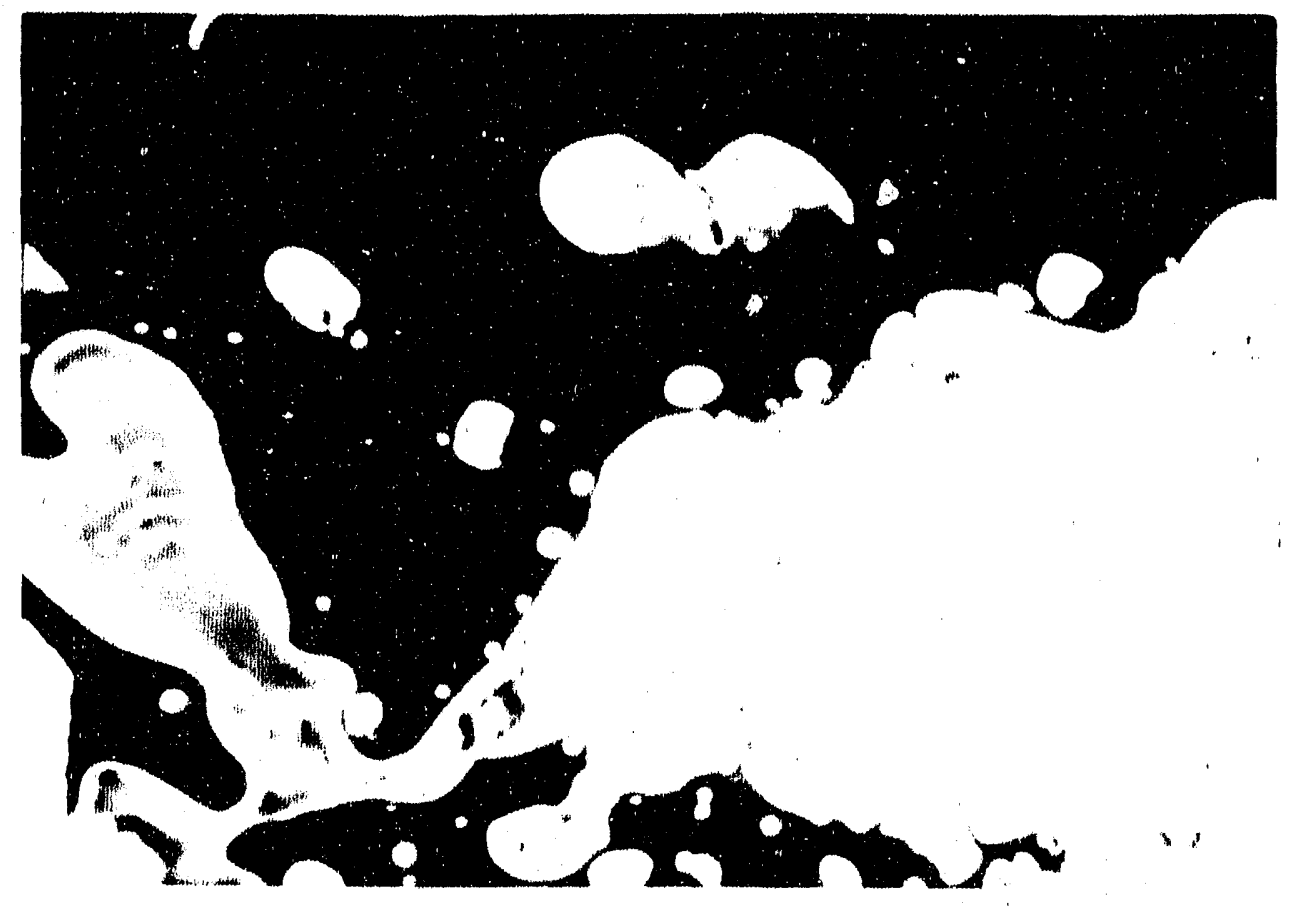

Figure 17: Close-up of steam front.

displacements. In general, a small advancement of the front often led to rapid condensation of a large part of the steam zone, as a result of heat transfer. Shortly thereafter, however, this event was quickly followed by a rapid burst of vapor-influx, which essentially restored to vapor occupation the previously condensed part. This cycle of advancement-vapor condensation-vapor replacement appeared to be the gerieral mechanism for the steam zone growth. In no instance did we observe a smooth frontal displacement of the typical water-oil interface. Also, in contrast to what normally expected, steam zones were not smooth and "flat", but rather finger-like, as they followed the flow paths of the condensed water. This process is mainly controlled by heat transfer, viscosity reduction, injection rates and temperatures. Therefore, our observations cannot be generalized without due caution.

Although most of the steam front is a water vapor-water liquid interface, a large fraction on the lateral sides consists of water vapor-oil interfaces (Figure 18). In fact, disconnected and stationary steam bubbles were frequently observed. Most of these dynamics are different from ordinary HeleShaw displacements. Indeed, the condensation and vapor growth phenomena in the above cycles involve flow rates high enough for inertia effects not to be negligible. This complex mechanism is currently under investigation. 


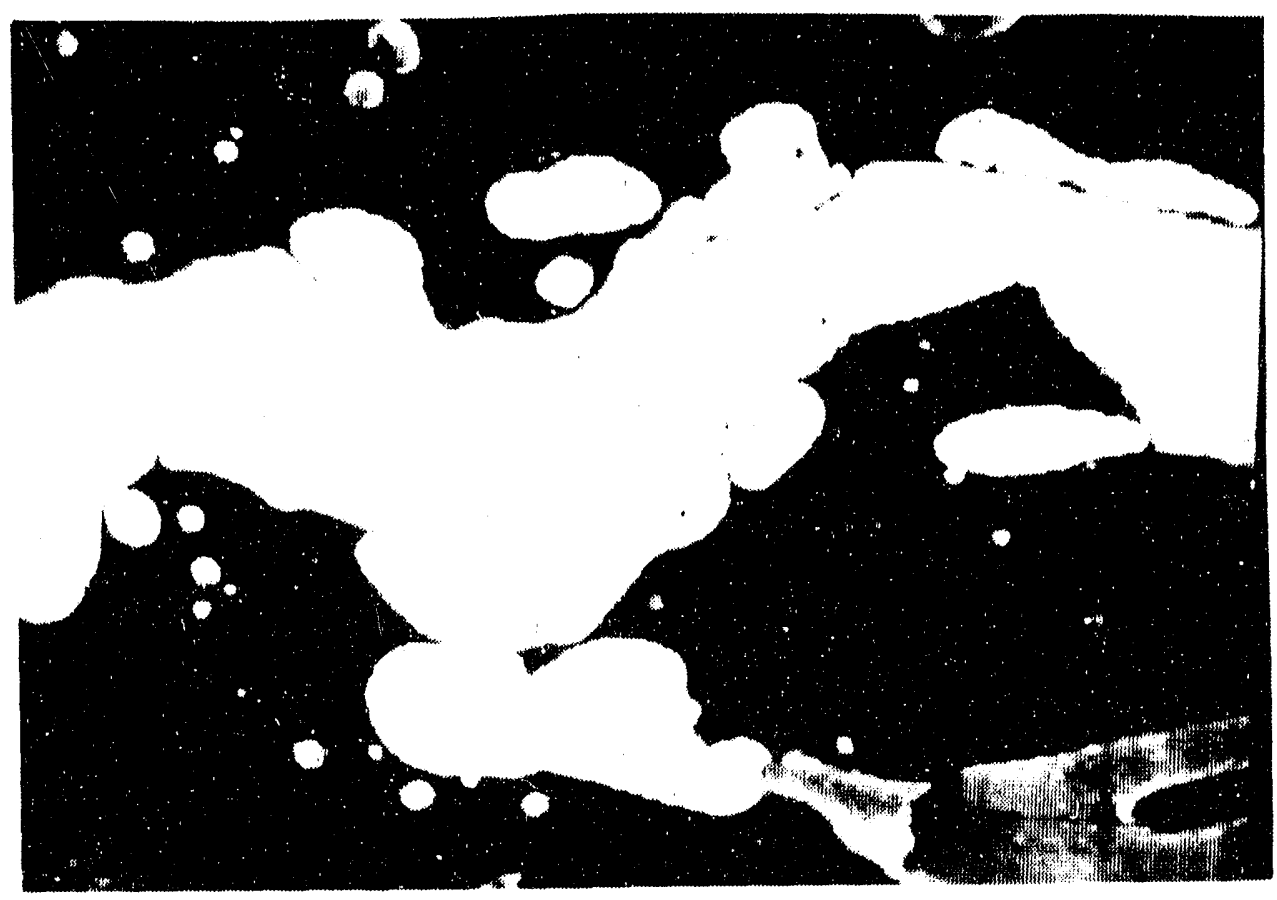

Figure 18: Water vapor-oil interface.

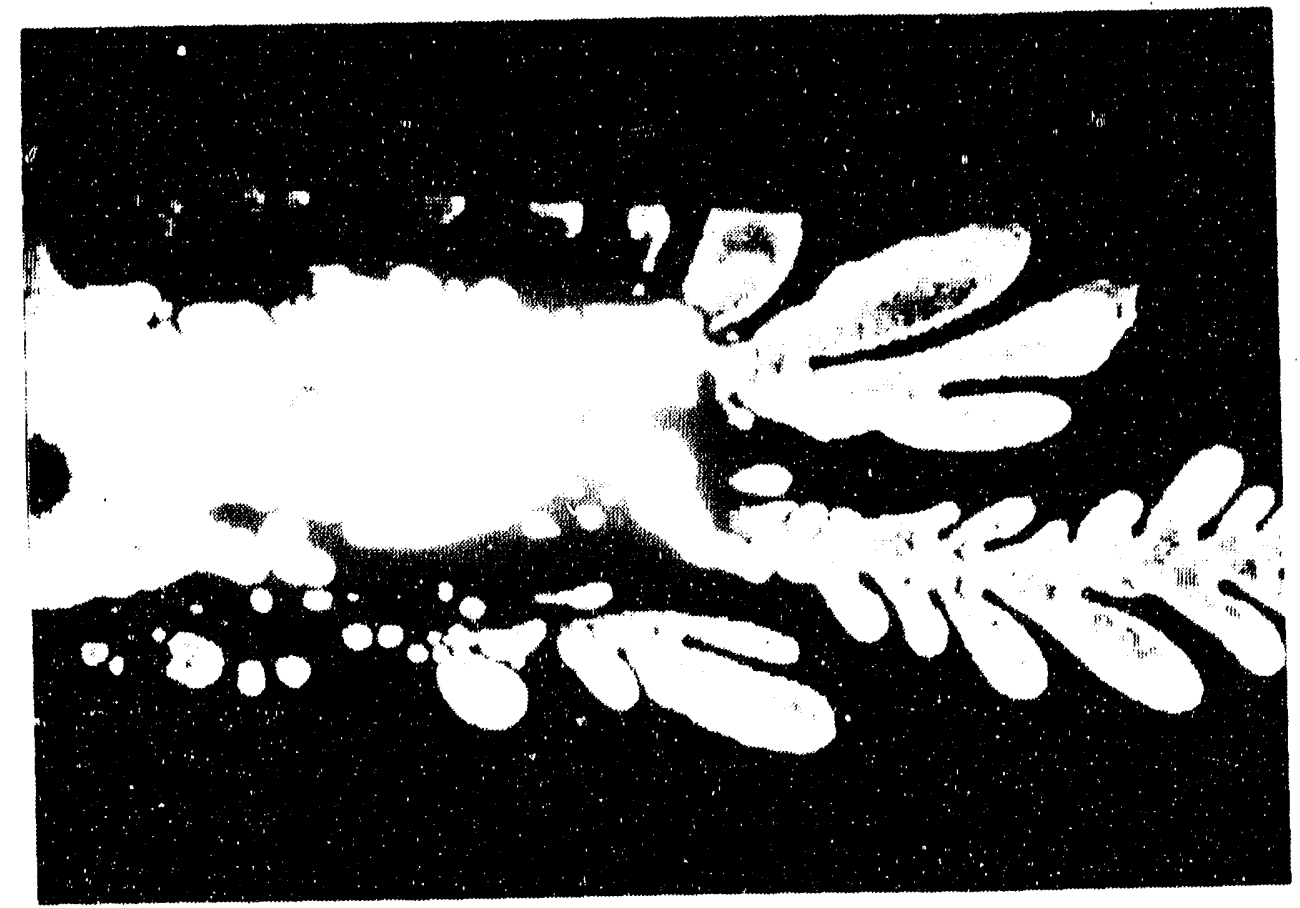

Figure 19: Finger after breakthrough. 


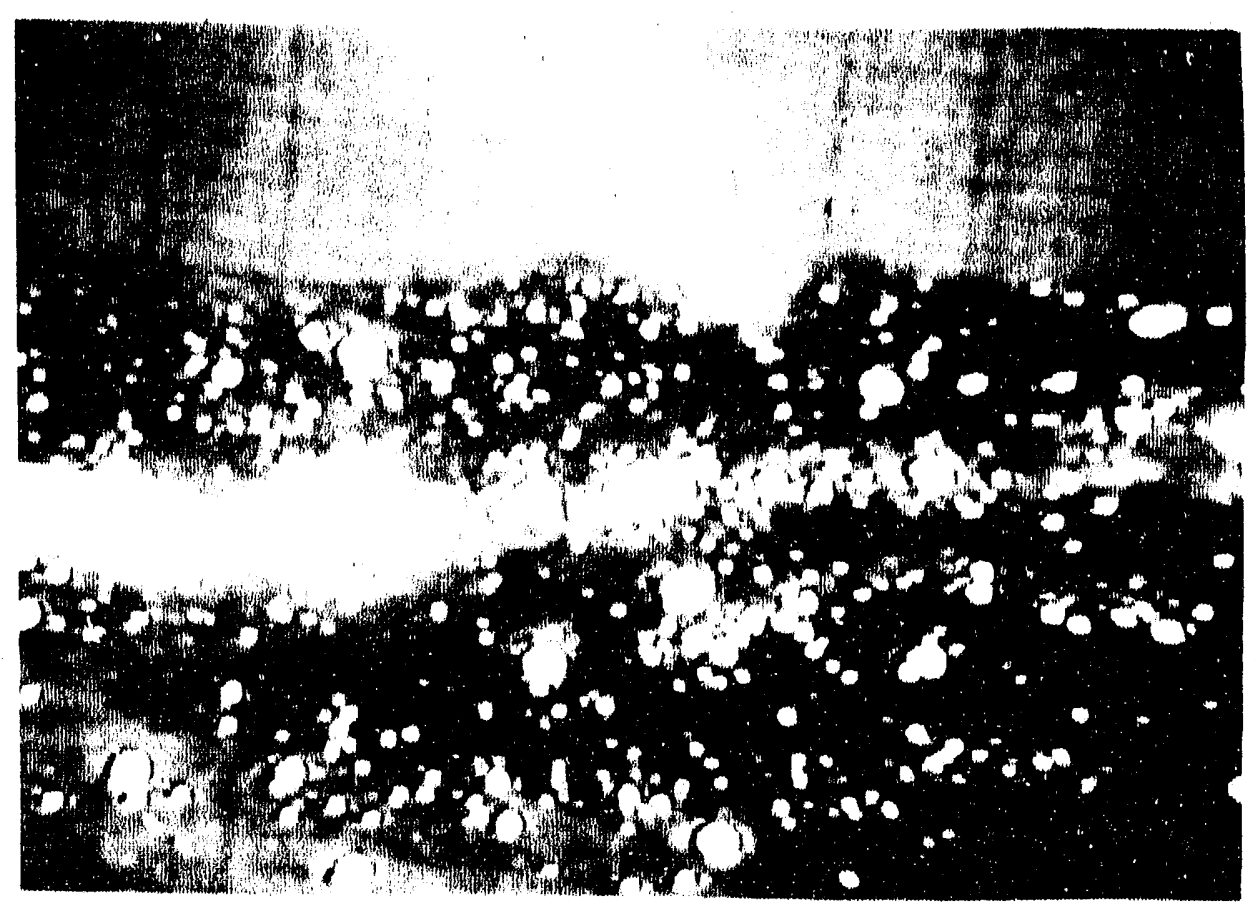

Figure 20: Emulsification of oil.

Breakthrough occurs when the fastest among the competing fingers reaches the production end. A typical picture after this time is shown in Figure 19. Important observations during this period were the following: Steam flowed mostly along the path connected to the production well. During this process, substantial emulsification of oil also occured (Figure 20). After breakthrough, a large fraction of the oil produced was in the form of emulsion. In our experiments, emulsification was enhanced by a rather unusual rheological response of Dutrex. Soon after water breakthrough, the connected finger started to continuously thin, until it eventually fragmented at one or several points and became disconnected. Figure 21 is at a stage just prior to this event. A different finger then started growing following a usual displacement process, until breakthrough of that path was also reached. Soon after, the process of thinning commenced again until eventual disconnection and the process was repeated again. These phenomena are not due to the injection of steam, but they are associated with the particular oil, and they were also observed in an ordinary waterflood.

A possible explanation for this behavior can be offered it we considered Dutrex to be viscoelastic. Before breakthrough, the fluid pressure is relatively high, due to the high viscosity. Upon water breakthrough, pressures decrease in both fluids. The oil responds to the pressure decrease by expanding following a relaxation process. This results into the thinning and shrinkage of the 


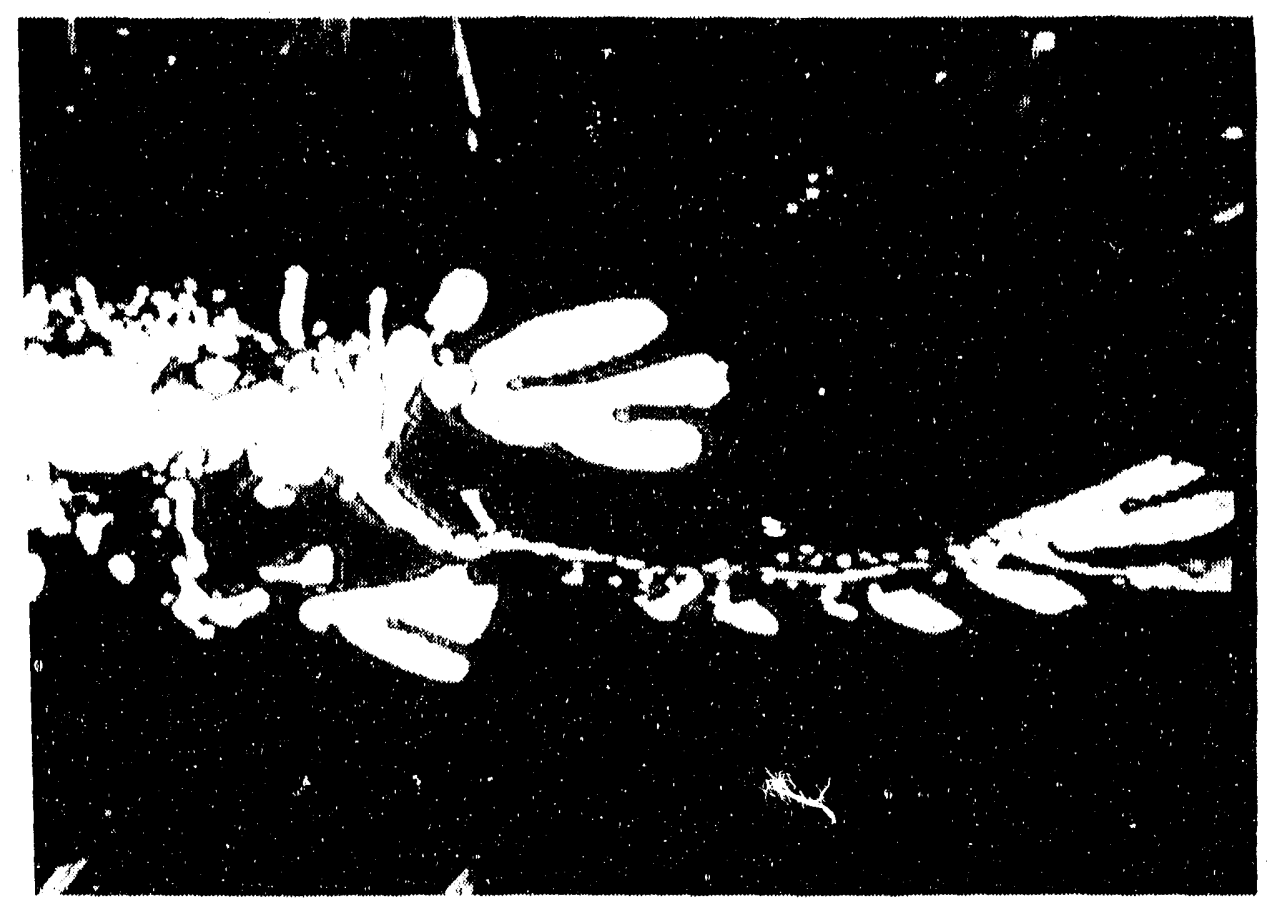

Figure 21: Finger thinning.

producing water finger until disconnection. The process is then repeated. This rough qualitative argument explains the gross behavior reported. However, for a more convincing interpretation, a model is needed. Work in this area is planned.

A comparison between steam and cold water displacement is shown in Figure 22 for the same water injection rates. Typical finger patterns before breakthrough (Figure 23) show characteristic tip-splitting. As pointed out above, residual oil films coat the glass plates. Upon breakthrough, the same response previously noted sets in. Figure 24 shows the finger pattern after considerable thinning and just prior to disconnection. These phenomena were periodically repeated. Significant differences with the steam injection runs involved the presence of residual films and the lack of emulsification in the case of water injection.

Effects of injection rate were also considered, by carrying a steam injection run at $1 \mathrm{cc} / \mathrm{min}$. As anticipated, the decrease in the rate resuits into a smaller steam zone area, which did not propagate significantly far into the cell. Otherwise, phenomena qualitatively similar to the previous were observed.

Finally, a comparison of the displacement process involving light (mineral) oil was also undertaken. Results of stearn injection at the rate of $3 \mathrm{cc} / \mathrm{min}$ and at the temperature of $230^{\circ} \mathrm{F}$ are 


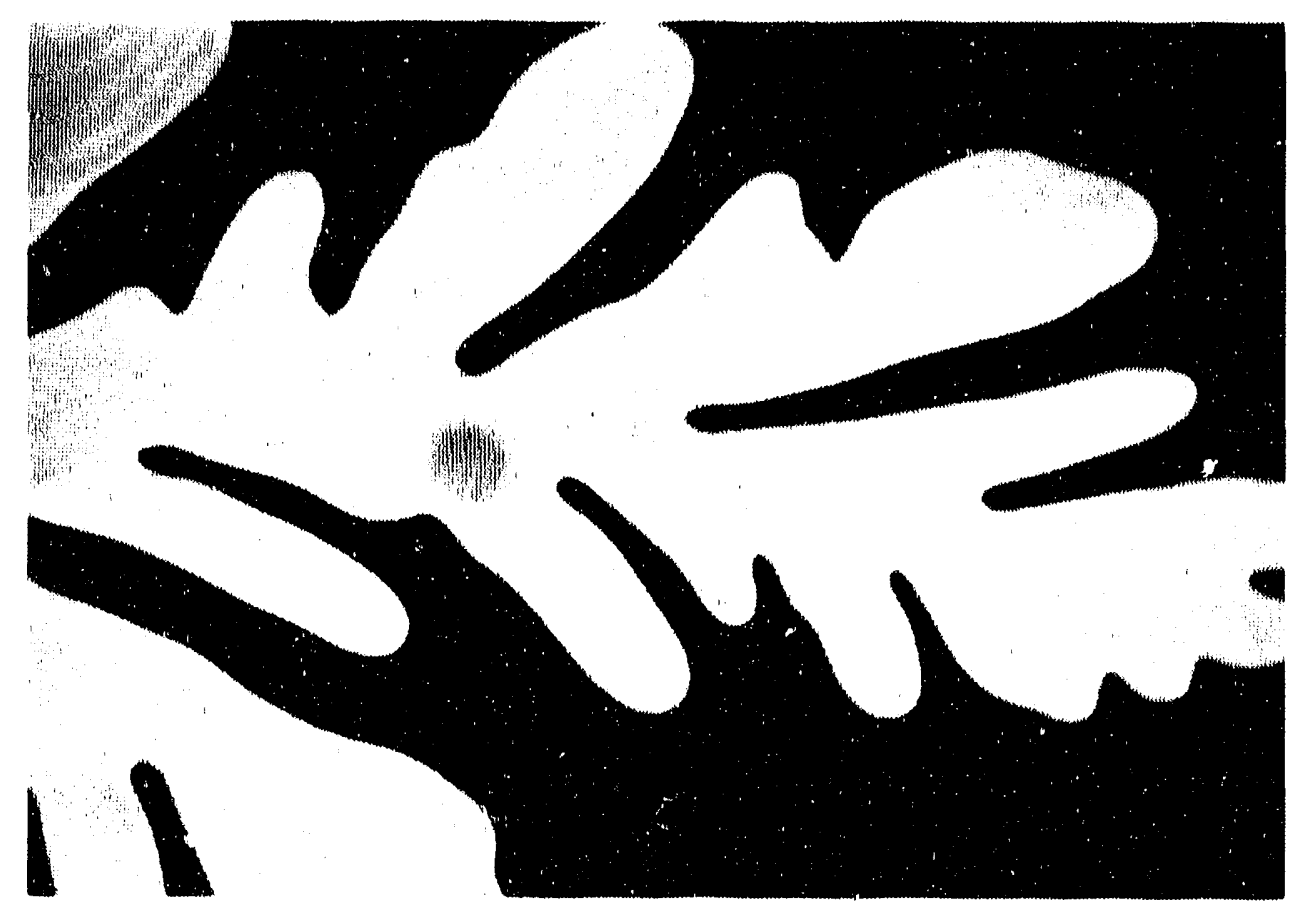

Figure 22: Oil displacement by water injection.
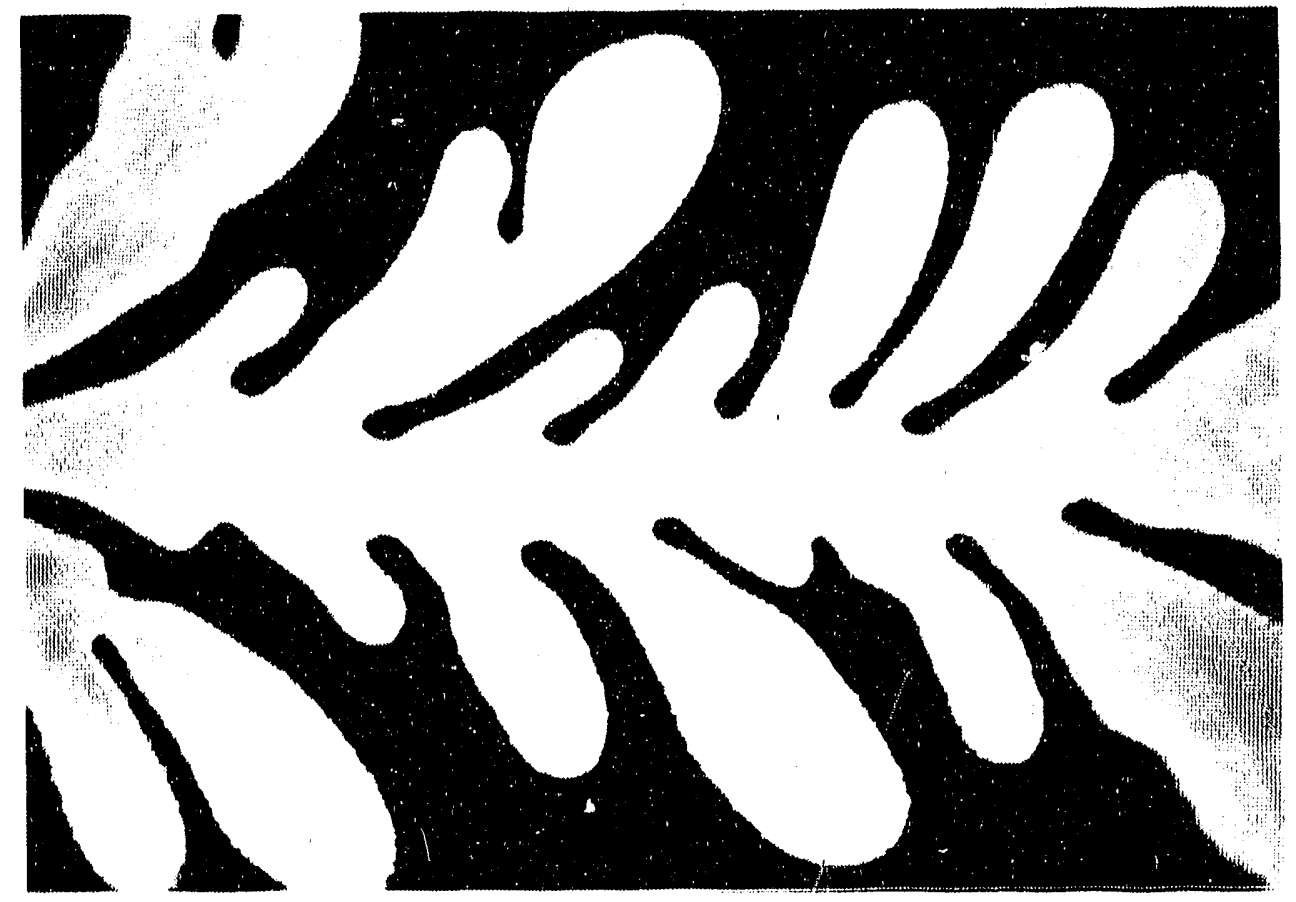

Figure 23: Tip-splitting in waterflood. 


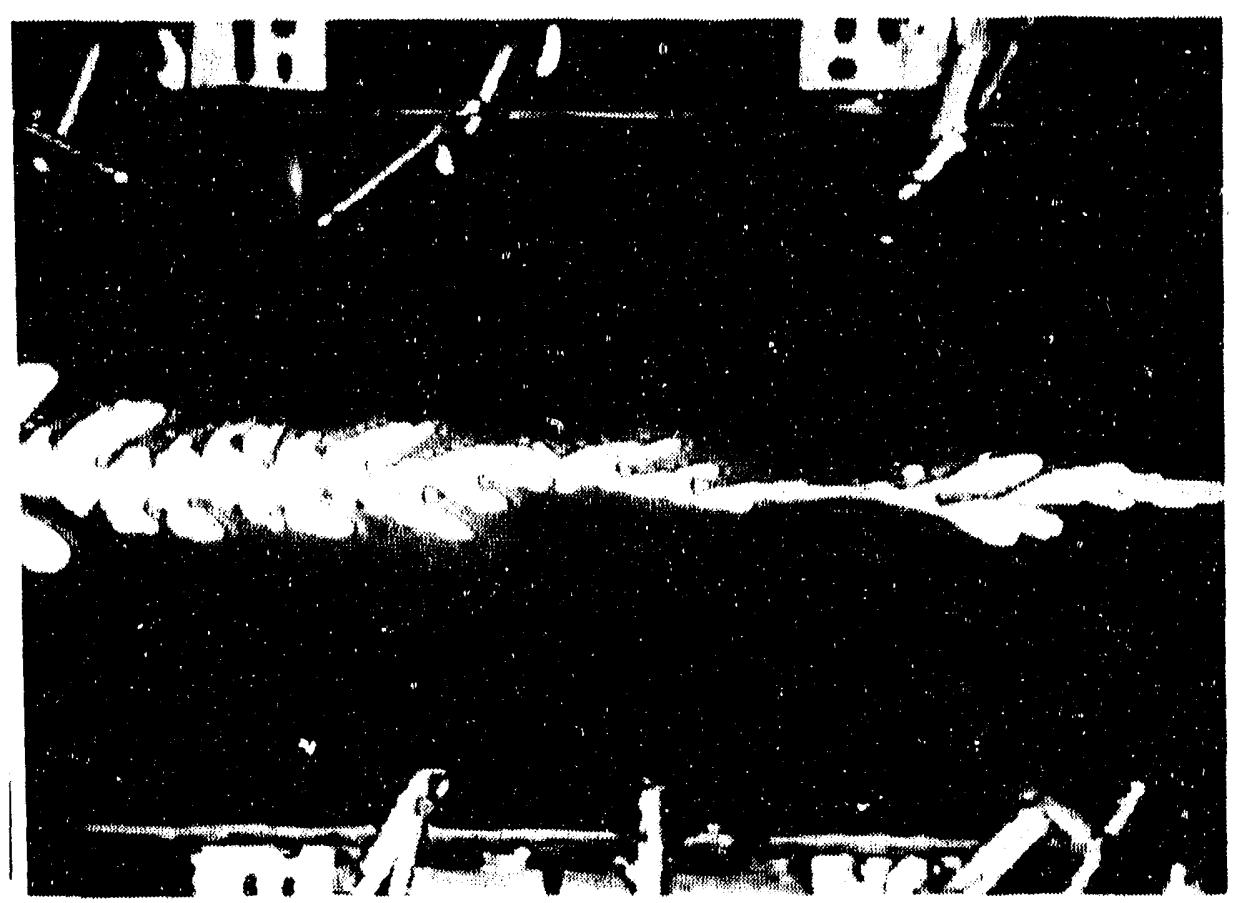

Figure 24: Finger pattern after thinning.

shown in Figure 25. Several significant differences were apparent. First, the degree of tip splitting was considerably less, as anticipated due to the lesser viscosity contrast in the light oil case. Second, no residual oil films were noted in the displacement by either water liquid or b steam. This observation was verified by cold water injection experiments also. Third, the steam front did not follow the path of the condensed water fingers, as in the heavy oil case, but the steam zone expanded almost uniformly (compare with Figure 26, at a later stage of injection). This behavior is consistent with and can be attributed to the higher mobility of the displaced fluid in the case of light oil. Finally, the rheological behavior of the mineral oil was Newtonian throughout the process. Upon breakthrough, the water finger width remained essentially constant and provided the main flow path for the injected water.

\subsubsection{Visualization in Glass Micromodels}

The second set of visualization experiments involved steam injection in glass micromodels. We used two kinds, one consisting of a simple arrangement of dead-end pores and pore doublet model, Figure 13, and another representing a fracture network obtained by the methods of Chapter 5 .

Various stages of the injection process in the first micromodel are shown in Figure 27. Steam at 


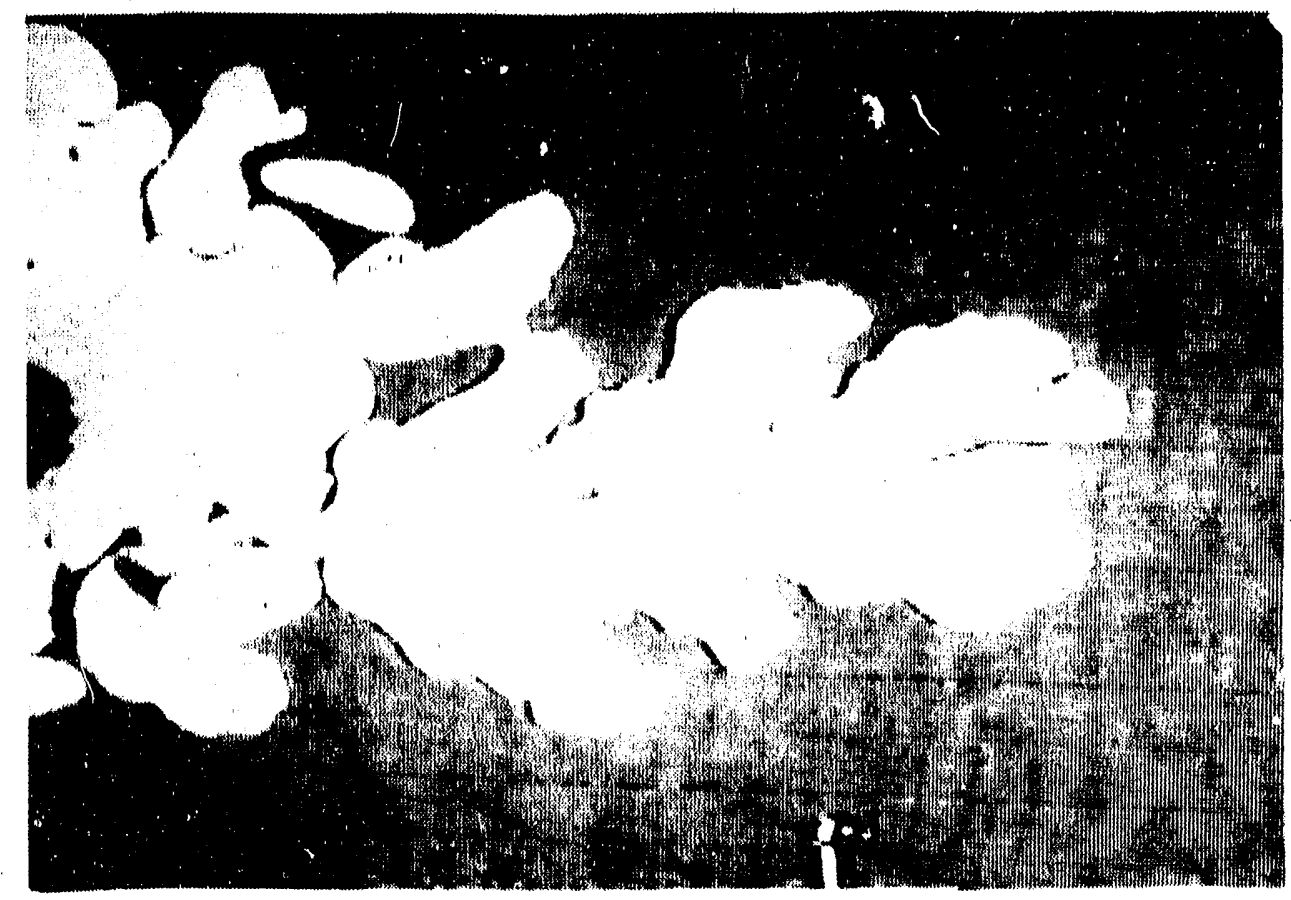

Figure 25: Mineral oil displaced by steam-Initial stage.

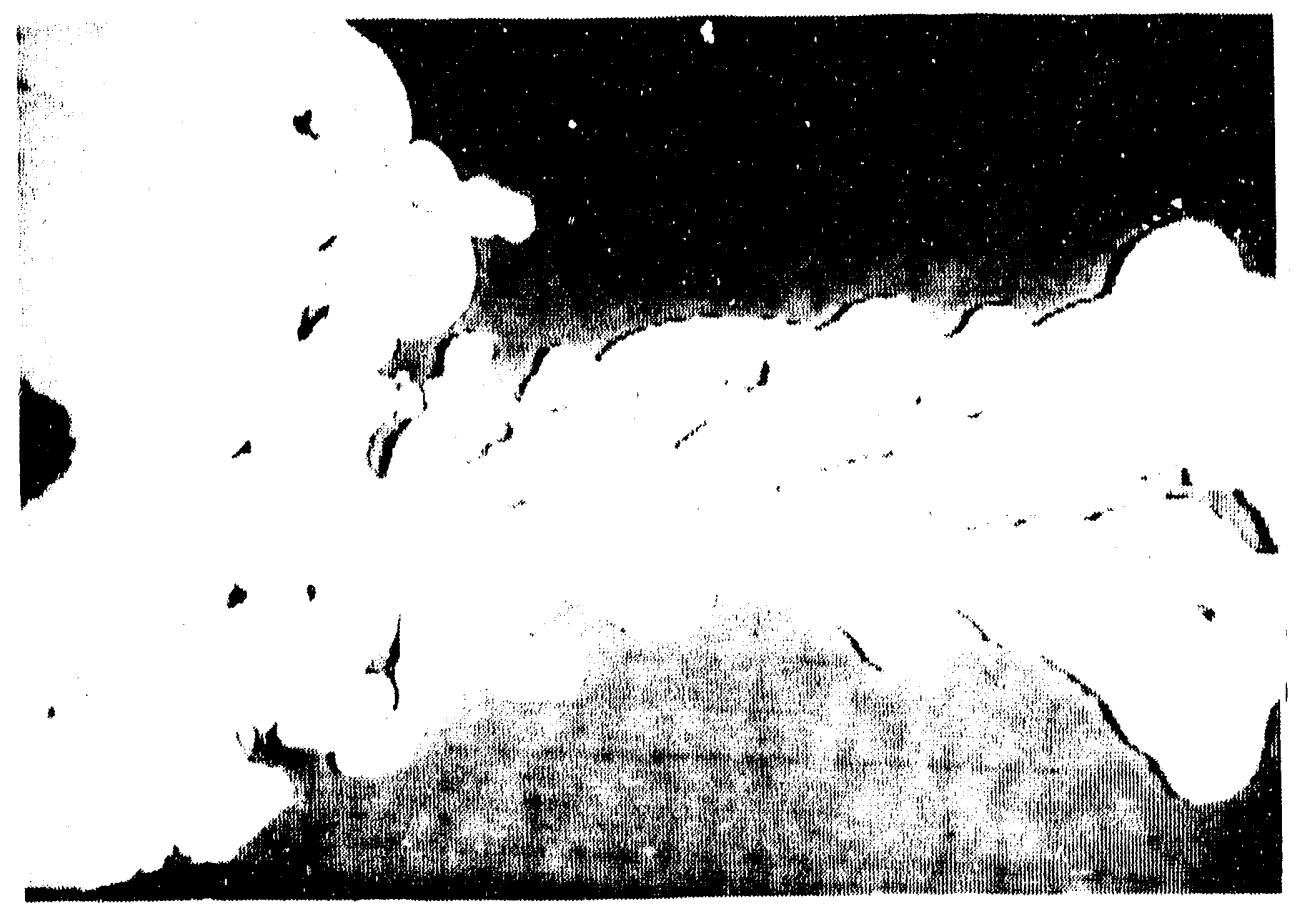

Figure 26: Mineral oil displaced by steam-Later stage. 


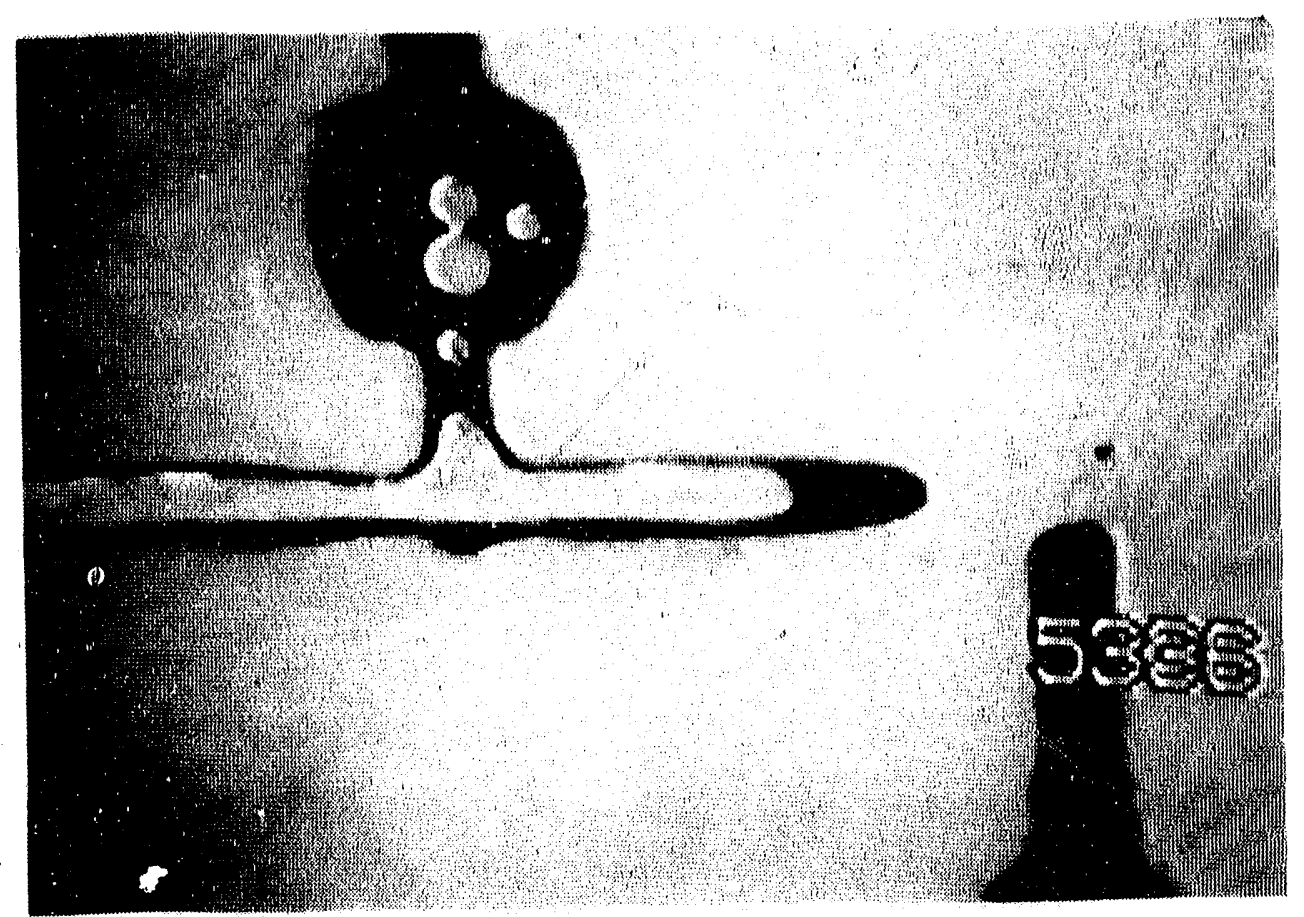

Figure 27: Steam injection into glass micromodel.

the rate of $1 \mathrm{cc} / \mathrm{min}$ and injection temperature of $220^{\circ} \mathrm{F}$ is injected from left to right and displaces mineral oil. The dark (red) phase in Figure 27 is oil, the transparent (not visible) phase is water liquid, and the visible bright white is water vapor. Displacement first occurs through the main pore conduit connecting inlet to oulet. This process does not occur smoothly, but involves growth, condensation, momentary steam bubble collapse and bubble restoration, following similar behavior as observed in the Hele-Shaw cell. Some penetration into the dead-end pores also occurs, usually resulting into bubble snap-off (Figure 28). Typically, oil film surrounds the vapor bubble. However, water liquid is the phase that wets the glass.

The penetration of steam into the dead end pores and the pore doublet, which contain trapped oil, also cccur in a non-smcoth fashion. The vapor-oil interface advances momentarily, then condensation occurs and part of the bubble collapses, to be restored shortly thereafter by the additional influx of vapor. During the processes of meniscus invasion and meniscus withdrawal, oil films developed between water vapor and water liquid are responsible for the recovery of oil from dead-end pores (Figure 29). These films provide the outlet for the flow of oil during the meniscus invasion. Rouhgness flow of oil was also observed. These cycles of invasion-withdrawal- depletion are repeated constantly and persist until all trapped and bypassed oil is completely recovered. At the 


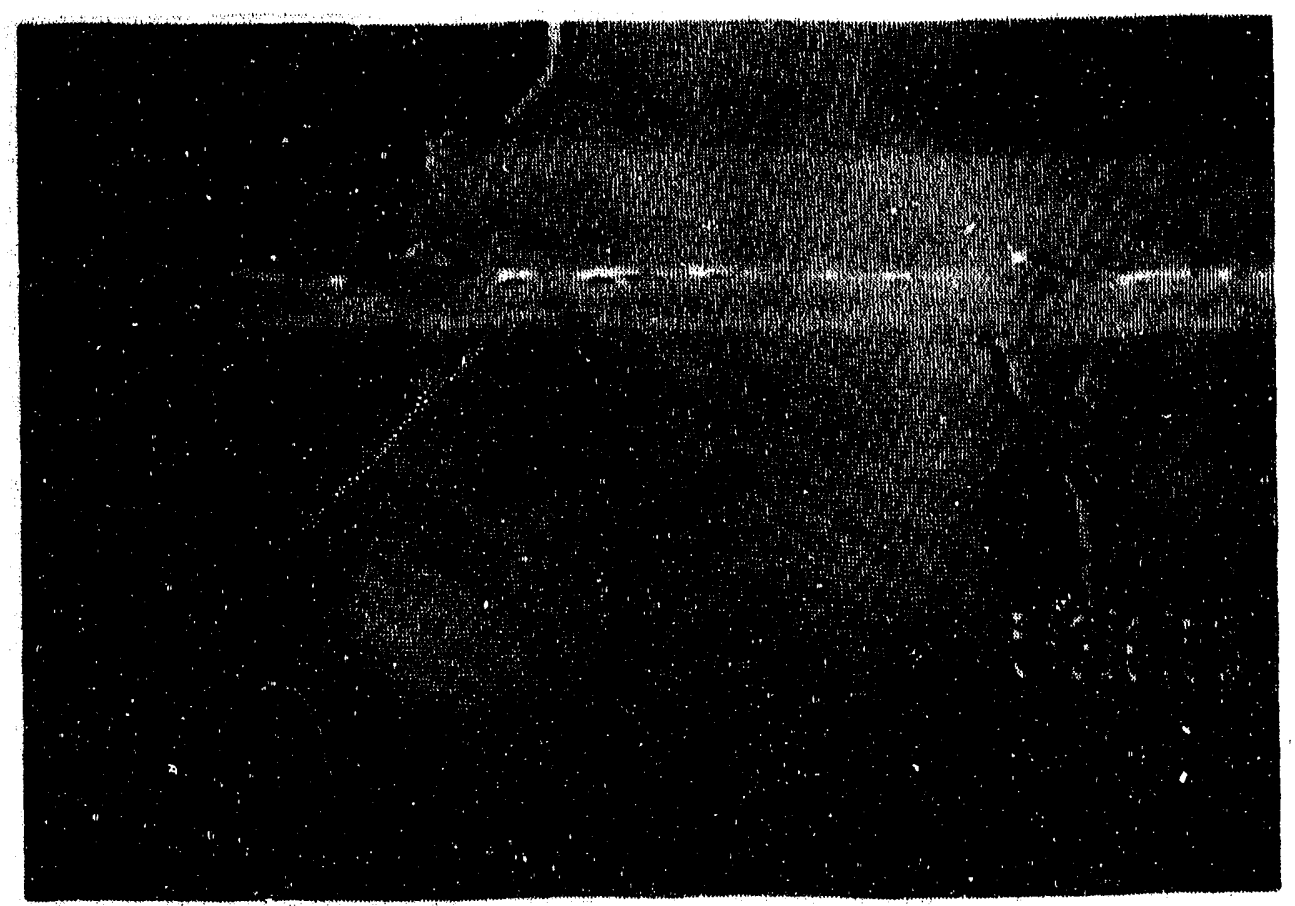

Figure 28: Vapor bubble snap-off.

conclusion of the process, all oil initially present is fully recovered.

Similar mechanisms were observed during the steam injection in the more complex model network of fractures shown in Figure 30 and Figure 31. Two successive stages of this process, where displacement occurs from left-to-right, are pictured. This network contains some large fractures running from injector-to-producer and a number of substantially smaller fractures, which are indirectly connected to the main conduits. At the first stage, oil displecement occurs along the main fractures, leading to oil entrapment in the maze of small fractures. Continuation of injection gives rise to the same phenomena reported above. A significant degree of film flow and flow by roughness was observed. Eventually, all trapped oil was displaced.

\subsection{CONCLUDING REMARKS}

The preliminary experiments reported above have probed a part of the complicated process of steam injection displacement. One of the most interesting results, common to both Hele-Shaw and micromodels, was the unsteady, almost flickering state of the steam front, and the mode of advancement by the cycle penetration-condensation-restoration. This is certainly to be affected by injection rate, heat transfer and flow mobilities. Our current work is aimed at the understanding 


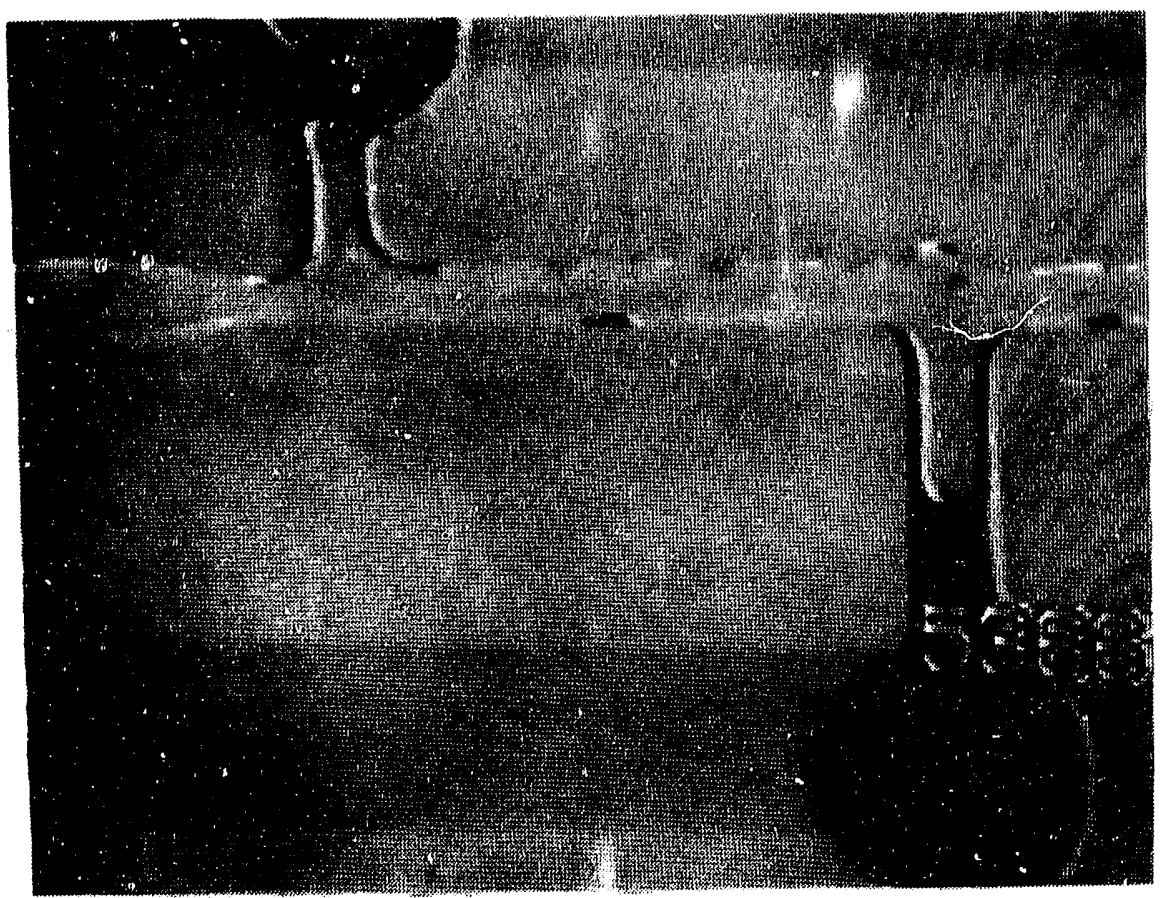

Figure 29: Oil film flow in a dead-end pore.

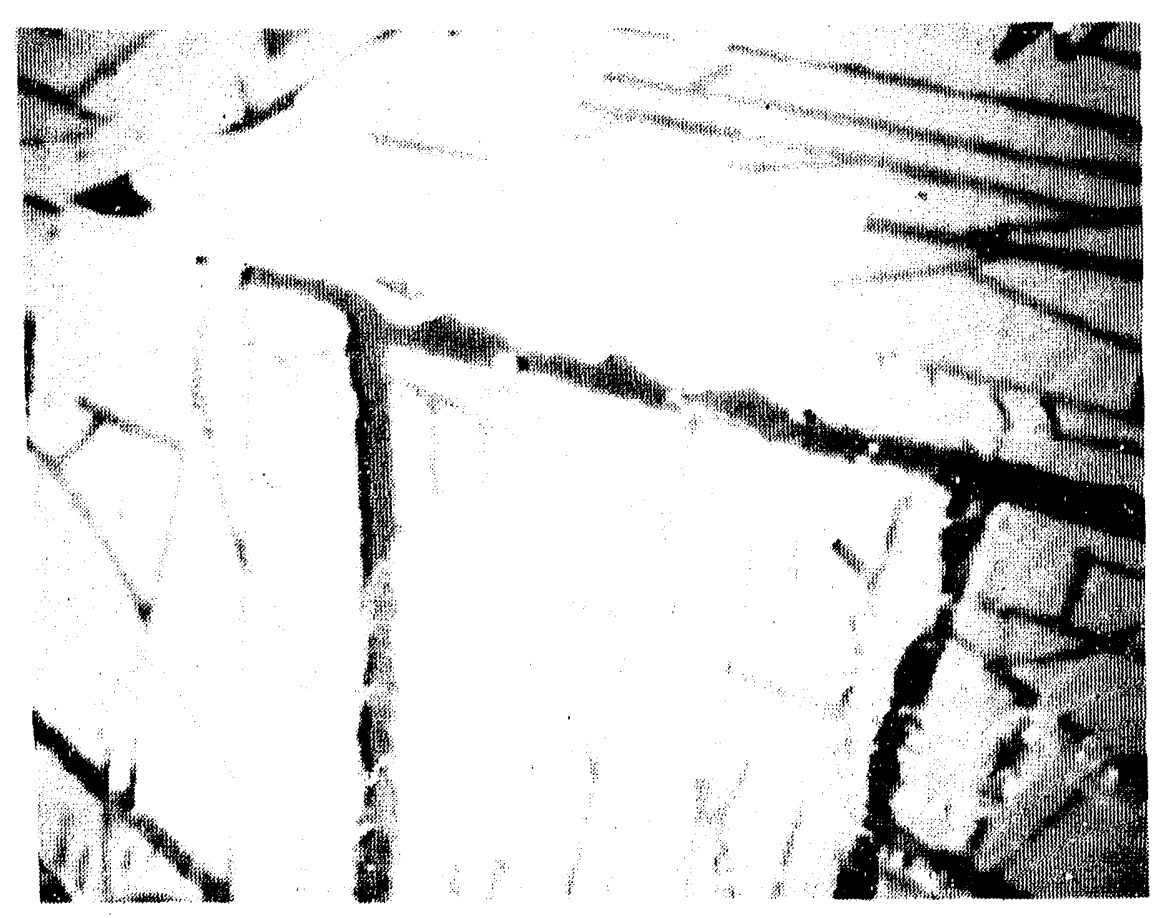

Figure 30: Steam injection into a fracture network-Initial stage. 


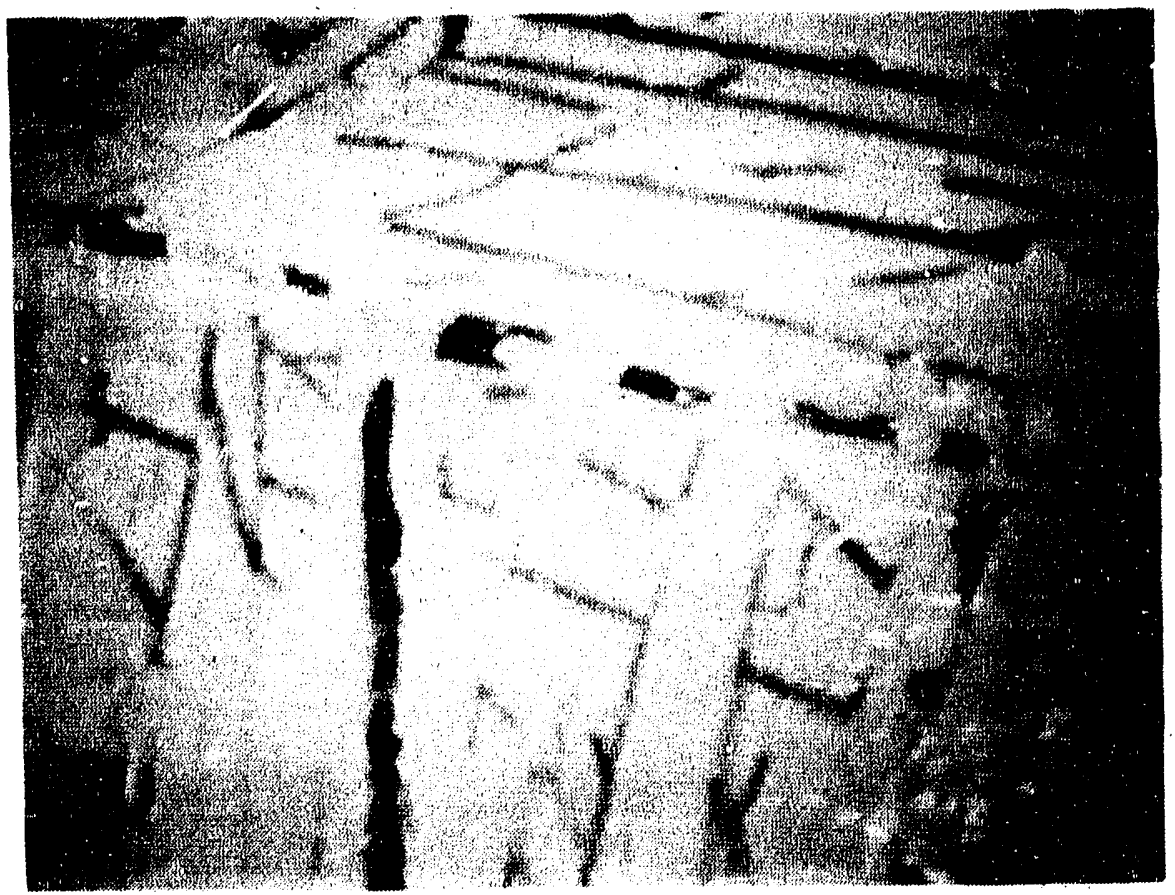

Figure 31: Steam injection into a fracture network-Later stage.

of this process.

For the low pressure, relatively high rate conditions of our experiments, injected steam was found to follow the path of the condensed water. The latter set the general displacement pattern, which in the case of heavy oil was highly fingered. It should be evident, that this result is a consequence of the particular combination of fluid flow and heat transfer in our experiments, thus any extrapolation must be done with caution. We also identified a rather unusual non-Newtonian behavior for Dutrex 739, a chemical taken to represent heavy oil in our experiments. Regardless of the particular aspects of the response observed, one may zeasonably conclude that the nonNewtonian rheology of many heavy oils is likely to lead to similarly novel behavior. This subject is further to be explored.

Our results in glass micromodels demonstrated aspects of three- phase, two-component flow. Film and roughness flow were found to be the main mechanisms for the recovery of trapped and bypassed oil. These preliminary findings are further studied by our group at present. 


\title{
4 STEADY-STATE, VAPOR-LIQUID CONCURRENT FLOW: RELATIVE PERMEABIL- ITIES AND END EFFECTS
}

\author{
M. Parlar, M. Zeybek, and Y.C. Yortsos
}

\subsection{INTRODUCTION}

The concurrent flow of a liquid and its vapor in a porous medium has been the subject of several experimental studies in the past. With main objective the estimation of vapor-liquid relative permeability pairs, simultaneous flows at steady-state and under adiabatic conditions have been investigated. Two flow configurations are typical: one involving the simultaneous injection of both vapor and liquid (e.g. steam-water), and another involving the injection of subcooled liquid only, that subsequently undergoes a phase change somewhere within the porous medium. Recent references for the former include [79], [56], [93] and [97]. The in-situ evaporation of a flowing liquid has been examined in an early paper by [54] using propane, and in a more recent application by [20] using water. In-situ phase change of a similar type, but with mass transfer the predominant transport process, has also been described ([32]).

While their relevance in modeling the condensing or evaporating flows, typical of thermal and geothermal processes, has not been rigorously established, and may in fact be questionable, steadystate relative permeability experiments are nonetheless attractive, due to their relative simplicity and for their own sake. Phase change introduces additional novel elements, absent from two-phase, two-component flows ("oil-water"), such as volumetric changes and heat transfer. Thus, despite the adiabatic conditions, fluid flow, phase change and heat transfer are nonetheless coupled to each other in all such experiments.

Analyses of the saturation, temperature and pressure profiles are usually obtained by decoupling fluid flow from heat transfer. An exception is the study by [97], who presented sample numerical results with the use of a general geothermal simulator previously developed ([68]). Capillary pressure is also typically omitted, although [79] make use of a Leverett $J$ function to relate vapor and liquid pressures. The latter authors implicitly assume that flat saturation profiles are established throughout the core. Although it is likely that approximations and assumptions made in previous studies were indeed justifiable, quantitative assessments have not been provided. Equally lacking are sensitivity analyses of the effects of the various parameters. 
The need for a more detailed description is underscored by the wide variation and scatter in relative permeability curves obtained by different investigators for relatively similar porous media. Instructive is the steam-water system, of interest both to thermal recovery of oil and to geothermal energy. The study in [93] report unusually low vapor (and high liquid) permeabilities, in all but a narrow region of low water saturation. In contrast, Counsil's (1979) data indicate an opposite effect, the vapor permeability being large (and the liquid permeability being small) in all but a narrow region of high water saturation. Trends somewhat similar to the latter were reported by [56], the vapor permeability exhibiting a sharp decrease above a certain water saturation. On the other hand, recently obtained data contradict most of the previous, in that the relative permeabilities reported are more representative of a gas-liquid system ([79] and[96]). Significant differences between the two pairs are claimed by the first authors, while a strong case is made by the latter authors for the similarity in relative permeabilities of vapor-liquid and gas-liquid pairs.

Theoretical work to model such flows at the micro-level has been limited. Besides the popular to the geothermal literature, but otherwise unsupported, model of straight-line relative permeabilities, the only work to address such issues was undertaken by [60], and by [106], in the context of steamwater, and solution gas drive, respectively. Both papers are restricted to the quasi-static limit, however, where temperature and pressure gradients are small, thus relative permeabilities are only saturation (and history) dependent. It must be noted that the model of [60] for drainage does indeed corroborate the findings of [79]. Nevertheless, additional work is needed to support this result and to resolve other issues. Chapter 2 of this report summarizes our recent efforts in that direction.

The wide experimental discrepancy calls to question the data interpretation, particularly in regions where the sensitivity to parameter valuess is high. Variables such as vapor (steam) quality, mass and energy injection rates, pressure, permeability and system length, all affect significantly saturation and temperature profiles. End effects are accompanied by evaporation-condensation phenomena adding yet another degree of complexity. A comprehensive analysis of such issues is not currently available. This forms the motivation for this study. We consider the simulation of steadystate, vapor-liquid, concurrent flow, as a result of either simultaneous injection or evaporation in situ. Following a conventional approach, we formulate a complete model of the process and we proceed to examine its sensitivity to various parameters. The work presented is a sequel of a 
previous study on countercurrent flows (Satik et al., 1991). As the object of this investigation is experiments in laboratory cores, end effects are addressed by generalizing recently developed methods ([104]) to single-component phases.

This chapter is organized as follows: The formulation of the problem for adiabatic injection in 1-D geometries is, first, presented. The approach is conventional, in that relative permeabilities that depend only on saturation are considered. Clearly, this constraints what follows to relatively small saturation and temperature gradients. For completeness, Kelvin effects are also included, although they are practically insignificant everywhere, but in the very low water saturation region (see also Satik et al., 1991). We subsequently examine saturation and temperature profiles across regions of permeability (capillary) heterogeneity, in order to generalize end effects (in the manner of [104]). Extrapolation of the results to infinitely sharp permeability contrast yields conventional end effects. The subsequent section deals with the construction of saturation-temperature paths (trajectories) from the solution of the flow problem. Simultaneous injection or injection of a single (subcooled) liquid phase are represented by different regions of the same trajectory. Finally, saturation and temperature profiles are discussed and sensitivity studies are performed in the final section.

\subsection{FORMULATION}

We consider the concurrent 1-D, horizontal flow of a single-component liquid and its vapor, under steady-state, adiabatic conditions. For purposes of generalizing end effects, a heterogeneous core is assumed. We proceed with mass balances for both vapor and liquid

$$
\begin{aligned}
& \frac{\partial}{\partial x}\left(\rho_{v} q_{v}\right)+\dot{m}=0 \\
& \frac{\partial}{\partial x}\left(\rho_{L} q_{L}\right)-\dot{m}=0
\end{aligned}
$$

where $\dot{m}$ is the condensation rate. 'The overall mass balance reads

$$
\frac{\partial}{\partial x}\left(\rho_{L} q_{L}+\rho_{v} q_{v}\right)=0
$$

and upon integration

$$
\rho_{L} q_{L}+\rho_{v} q_{v}=m_{L}+m_{v}=m_{i}
$$


where $m_{i}$ is the mass injection rate. The conventional expressions for Darcy's law are next taken

$$
\begin{gathered}
q_{v}=-\frac{k(x)}{\mu_{v}} k_{r v}(S) \frac{d P_{v}}{d x} \\
q_{L}=-\frac{k(x)}{\mu_{L}} k_{r L}(S)\left[\frac{d P_{v}}{d x}-\frac{d P_{c}}{d x}\right]
\end{gathered}
$$

A capillary pressure curve in terms of a Leverett $J$ function is assumed

$$
P_{c}=\frac{\sigma}{\sqrt{k}} J(S)
$$

while the vapor pressure is approximated by a Clausius- Clapeyron-Kelvin expression

$$
P_{v}=P_{v 0}\left(T_{r}\right) \exp \left[\frac{L_{v r}}{\tilde{R}}\left(\frac{1}{T_{r}}-\frac{1}{T}\right)-\frac{v_{L}}{R T} P_{c}\right]
$$

More accurate phase equilibria can be used, although expected gains are not significant. For numerical purposes, relative permeability and capillary pressure curves are approximated by the simple, ad hoc expressions of Table 2. For generality, Kelvin effects were also included above, although their contribution is quantitatively negligible (except in the region of low liquid saturation).

The energy balance completes the formulation

$$
\frac{\partial}{\partial x}\left\{\rho_{L} q_{L} h_{L}+\rho_{v} q_{v} h_{v}\right\}=\frac{\partial}{\partial x}\left(\lambda \frac{\partial T}{\partial x}\right)
$$

to yield upon subsequent integrałion

$$
\rho_{L} q_{L} h_{L}+\rho_{v} q_{v} h_{v}=\lambda \frac{d T}{d x}+C
$$

The constant of integration, $C$, is the enthalpy injection rate, and can be related to either injection or production values. For example, using injection data (subscript 0 ), we obtain

$$
\begin{aligned}
C= & m_{i} *\left\{\left(h_{L r}+\chi_{0} L_{v r}\right)+\left[c_{p L}-\chi_{0} *\left(c_{p L}-c_{p v}\right)\right] *\right. \\
& \left.\left(T_{0}-T_{r}\right)\right\}-\left.\lambda \frac{d T}{d x}\right|_{0}
\end{aligned}
$$

with $\chi_{0}$ denoting vapor quality at injection and where subscript $r$ indicates reference temperature. 


$$
\begin{array}{lll}
R_{h}=\frac{\lambda T_{r} \mu_{L}}{\sigma \sqrt{k^{*}} \rho_{L} L_{v r}} & R_{m}=\frac{m_{i} \mu_{L} L}{\sigma \sqrt{k^{*}} \rho_{L}} & R_{L}=\frac{\lambda T_{0} / L}{m_{i} L_{v r}} \\
R_{p}=\frac{P_{v_{0}}\left(T_{r}\right)}{\sigma / \sqrt{k^{*}}} & R_{\mu}=\frac{\mu_{L}}{\mu_{v}} & R_{\rho}=\frac{\rho_{v}}{\rho_{L}} \\
K=\frac{L_{v r}}{\tilde{R} * T_{0}} & \tilde{R}=\frac{R}{M} & k_{D}(\xi)=\frac{k(\xi)}{k^{*}} \\
r_{c h}=\frac{2 \sigma v_{L}}{R T} & b=\frac{r_{c h}}{2 \sqrt{k^{*}}} & \\
\Theta=\frac{T}{T_{r}} & \xi=\frac{x}{L}
\end{array}
$$

$$
\begin{aligned}
& k_{r L}=S^{2.35} \\
& k_{r v}=(1-S)^{2.35} \\
& J=1.417(1-S)-2.12(1-S)^{2}-1.263(1-S)^{3}
\end{aligned}
$$

Table 2: List of dimensionless parameters and variables 
After lengthy manipulations the above formulation is further recast in terms of two coupled differential equations, one for the saturation and another for the temperature distributioris. In the general case of variable permeability, the system has the form

$$
\begin{gathered}
\frac{d J}{d S} \frac{d S}{d \xi}=R_{m} \frac{F(\Theta, S ; \xi)}{H(\Theta, S ; \xi)} \\
\frac{d \Theta}{d \xi}=R_{m} \frac{G(\Theta, S)}{H(\Theta, S ; \xi)}
\end{gathered}
$$

where specific expressions for $F(\Theta, S ; \xi), G(\Theta, S)$ and $H(\Theta, S ; \xi)$ are given in Appendix. The notation is dimensionless, with the reference temperature $T_{r}$ and the core length $L$ normalizing temperature and distance, respectively.

The system (47)-(48) contains all significant effects, including capillarity, heat conduction and phase change, summarized for convenience in Table 2 in terms of dimensionless parameters. Numerical estimates for typical conditions corresponding to the data of [79] and [54] are given in Tables 3 and 4, for water and propane, respectively. Among the important variables are the macroscopic capillary number $R_{m}$, which scales rate, the parameter $C / m_{i}$, expressing enthalpy input per flowing mass, and the parameter $R_{h}$, representing the relative importance of heat conduction. In the absence of the latter or for high permeability media $\left(R_{h} \ll 1\right)$, saturation and temperature equations decouple from each other, an assumption often used.

The solution of (47)-(48) is not as straightforward as it might appear. Solving the problem given inlet saturation and temperature values is highly sensitive to the initial conditions, particularly near inlet saturations for which flat profiles are expected. This sensitivity is not specific to this problem, but also exists for non-condensing flows as well ([105]). A backwards integration is thus necessary, requiring in turn that conditions at the outlet end be specified. This necessitates that end effects be addressed.

Conventional end effects correspond to large changes (orders of magnitude) in permeability over a small space interval. This can be considered as a special limit of the more general case of capillary heterogeneity across an arbitrary permeability profile. The results obtained from such study may then be applied to the special case corresponding to inlet or outlet end effects. This approach is taken in the next section. 
DIMENSIONAL VARIABLES:

Total Mass Flux, $m_{i}(\mathrm{gr} / \mathrm{hr})=120$

Exit Temperature, $T_{1}\left({ }^{\circ} \mathrm{C}\right)=100$

Reference Temperature, $T_{r}\left({ }^{\circ} \mathrm{C}\right)=100$

Permeability, $k^{\circ}$ (darcy) $=0.5$

Cross Sectional Area, (sq.cm) $=20.268$

Heat Input Rate, $C$ (btu/hr) $=100$

Thermal Conductivity, $\lambda\left(\frac{W}{m \cdot K}\right)=2.5$

Interfacial Tension, $\sigma\left(\frac{\text { dymet }^{\prime}}{\mathrm{sm}}\right)=58.91$

Core Length, $L(\mathrm{~cm})=50$

\section{DIMENSIONLESS PARAMETERS :}

$$
\begin{aligned}
& b=0.000504 \\
& K=13.10923 \\
& R_{\mu}=22.41270 \\
& R_{p}=0.000625 \\
& R_{p}=1.21621 \\
& R_{m}=0.058034 \\
& R_{h}=0.002917 \\
& R_{L}=0.050265
\end{aligned}
$$

Table 3: Typical values of variables and parameters for water vapor-liquid flow 


\title{
DIMENSIONAL VARIABLES:
}

\author{
Total Mass Flux, $m_{i}(\mathrm{gr} / \mathrm{hr})=4699.2$ \\ Exit Temperature, $T_{1}\left({ }^{\circ} \mathrm{C}\right)=21.1\left(70^{\circ} \mathrm{F}\right)$ \\ Reference Temperature, $T_{r}\left({ }^{\circ} \mathrm{C}\right)=35.56\left(96^{\circ} \mathrm{F}\right)$ \\ Permeability, $k^{\circ}$ (darey) $=0.9$ \\ Cross Sectional Area, (sq.cm) $=22.018$ \\ Heat Input Rate, $C$ (btu/hr) $=2720.3$ \\ Thermal Conductivity, $\lambda\left(\frac{W}{m \cdot X}\right)=2.5$ \\ Interfacial Tension, $\sigma\left(\frac{\text { dymec }}{c m}\right)=5.5$ \\ Core Length, $L(\mathrm{~cm})=152.4$
}

\section{DIMENSIONLESS PARAMETERS :}

$$
\begin{aligned}
& b=0.000199 \\
& K=5.49054 \\
& R_{\mu}=12.04819 \\
& R_{p}=0.056749 \\
& R_{p}=213.11244 \\
& R_{m}=36.4553 \\
& R_{h}=0.0974 \\
& R_{L}=0.0027
\end{aligned}
$$




\subsection{CAPILLARY HETEROGENEITY}

The saturation profiles for the 1-D flow of two immiscible, non-condensing phases in heterogeneous cores were studied in two recent publications ([103], [104]). The results showed that, as a rule, the wetting phase saturation responds to a capillary (permeability) heterogeneity by exhibiting a $\Lambda$-like kink, in the case of a step-like permeability increase, and a V-like kink in the opposite case. In principle, similar effects would be anticipated for the present case. However, the coupling with the energy balance, and the temperature response itself, need further study.

Using backwards integration, the set (47)-(48) was numerically simulated for the standard conditions of Table 3 and for a step-like increase in permeability (Figure 32). The saturation response has features similar to those for flow of non-condensing phases ([104]): There is a build-up of the wetting (liquid) saturation in the region preceding the heterogeneity, and a subsequent decline to the original level, the latter variation confined entirely within the region of permeability change. Furthermore, for the present case, the saturation change is accompanied first by condensation and increased temperature gradients, and subsequently by evaporation and a gradual decrease of the temperature gradient.

Analogous behavior is shown in the case of permeability decrease (Figure 33). Now, the liquid saturation decreases in the region preceding the heterogeneity, giving rise to evaporation and lower temperature gradients. As in the previous, the original saturation value is rapidly restored by subsequent increases in saturation and temperature gradients, all taking place inside the heterogeneity interval.

It was previously stated that this response exhibits all the characteristics of the two-component, two-phase problem ([104]). This should be the case in the limit of negligible conduction $\left(R_{h} \ll 1\right)$, negligible Kelvin effects, and constant latent heat $L_{v}$, where the two problems become mathematically identical. Indeed, at such conditions, the problem (47)-(48) decouples and takes the form

$$
\begin{gathered}
\epsilon \frac{d J}{d S} \frac{d S}{d \xi}=\left\{\frac{E}{W R_{m}}-F_{L}+\frac{\epsilon F_{L} k_{r v} J}{2 \sqrt{k_{D}}} \frac{d k_{D}}{d \xi}\right\} \div \\
k_{r v} F_{L} \sqrt{k_{D}} \\
\epsilon \frac{d A}{d \xi}=-\frac{\left(1-\left(E / W R_{m}\right)\right)}{k_{r v} k_{D}}
\end{gathered}
$$



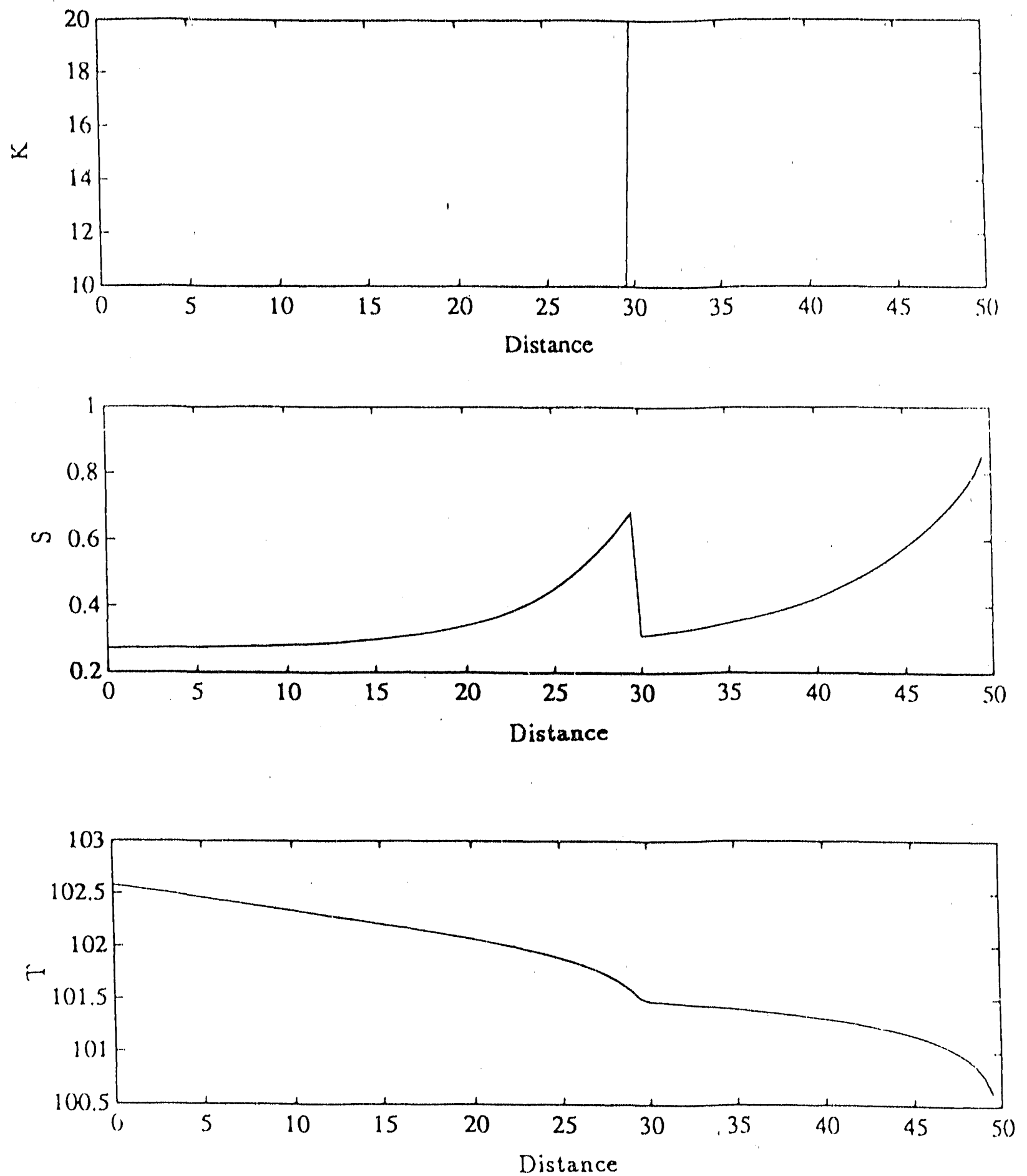

Figure 32: Capillary heterogeneity: saturation and temperature response for a step increase in permeability. 

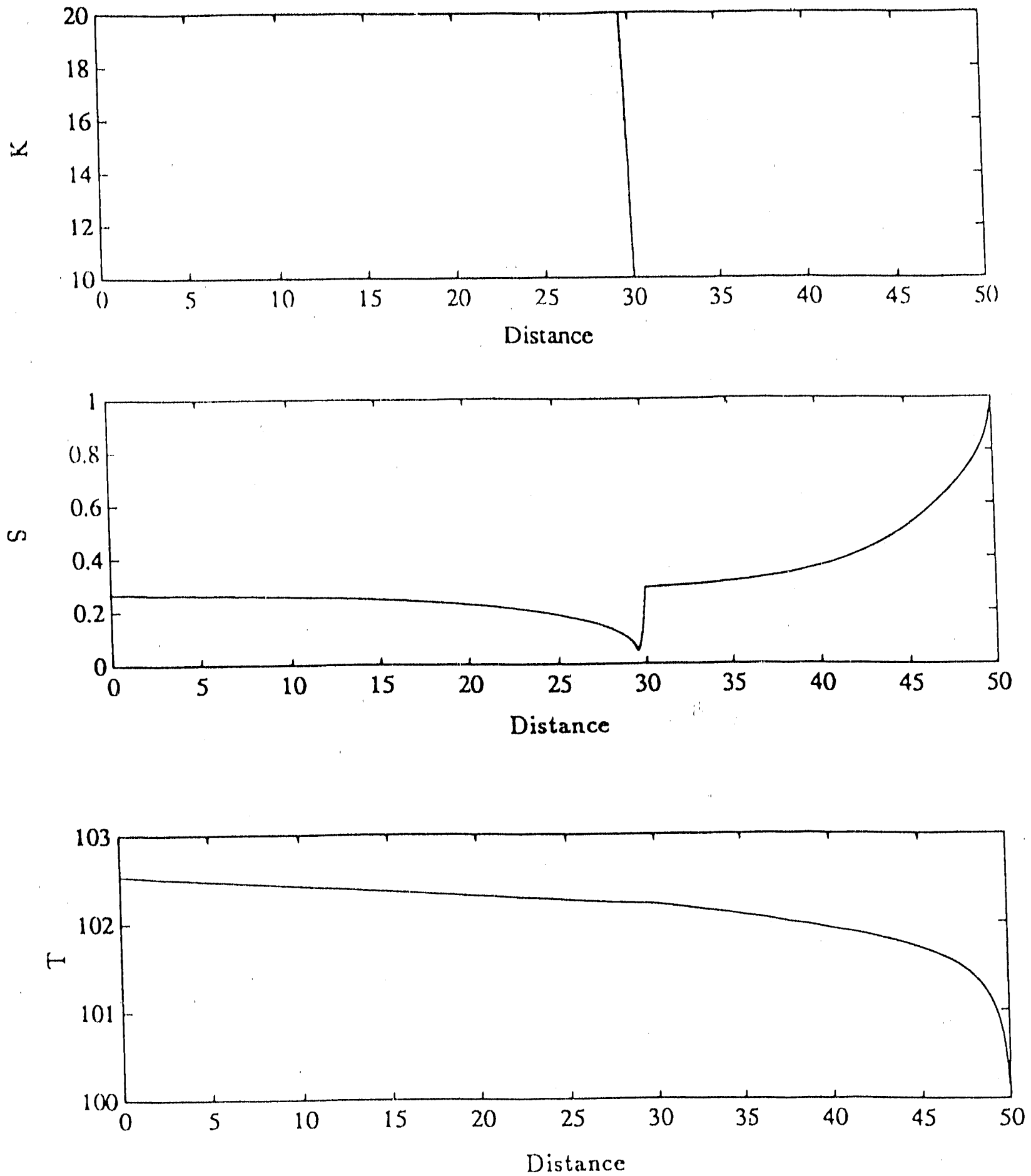

Figure 33: Capillary heterogeneity: saturation and temperature response for a step decrease in permeability. 
where parameter $\epsilon=R_{\mu} R_{\rho} / R_{m}$ as the relevant macroscopic capillary number. In the above the fractional flow $F_{L}$ expresses a flowing liquid mass fraction,

$$
F_{L} \equiv \frac{1}{1+\left(k_{r v} / k_{r L}\right) R_{\mu} R_{\rho}}
$$

the viscosity ratio being replaced by the ratio of kinematic viscosities $R_{\mu} R_{\rho}$. By expressing the injected fraction in terms of the injected vapor quality, $\chi_{0}$

$$
\frac{E}{W R_{m}}=\frac{m_{i} h_{v}-C}{m_{i} L_{v}} \simeq 1-\chi_{0}
$$

the saturation equation (49) is identical to that for non-condensing flow ([105]). The energy balance (50) expressed in terms of the normalized vapor pressure, $A$, can subsequently be solved. At such conditions, all the saturation features follow readily by the analysis in [105].

Extrapolation of the results to conventional outlet end effects is straightforward. Assuming that a single-phase region does not develop prior to exit, the "infinitely" sharp increase in permeability induces a maximum liquid saturation buildup, therefore a complete condensation at the outflow end, which is accompanied by sharp temperature gradients and a relatively significant temperature decrease. Phase equilibria regarding exit pressure and temperature are implied in the above. When this is not the case (single phase flow conditions with either superheated vopor or subcooled liquid), end effects are absent. Extrapolation to inlet end effects is also straightforward, although not strictly applicable. Nonetheless, one tentatively expects evaporation and flat temperature profiles prior to inlet. Outlet end effects for typical conditions in homogeneous cores are illustrated in Figure 34.

Subsequently, the effect of thermal conductivity was considered, From the definition of $R_{h}$, thermal cond activity can be signifi"ant at low enough permeabilities. Figure 35 shows the response obtained for a several-fold increase in conductivity, other parameters being identical to those in l'igure 32 , with the exception of the permeability variation. We observe that while temperature profiles become smoother, as expected, the saturation response is significantly affected, the capillary heterogeneity being felt over a region substantially larger than in the small conduction case. Likewise, outflow end effects become substantial. The conduction term not being negligible, the postulated analogy in (49)-(52) with the results of $[105]$ is no longer valid, and further study is needed. We mus'. struss that althrugh the profiles in Figure 35 are for high conductivities, similar rffects are also expected for typical values in low permeability cores, in view of the scaling properties 

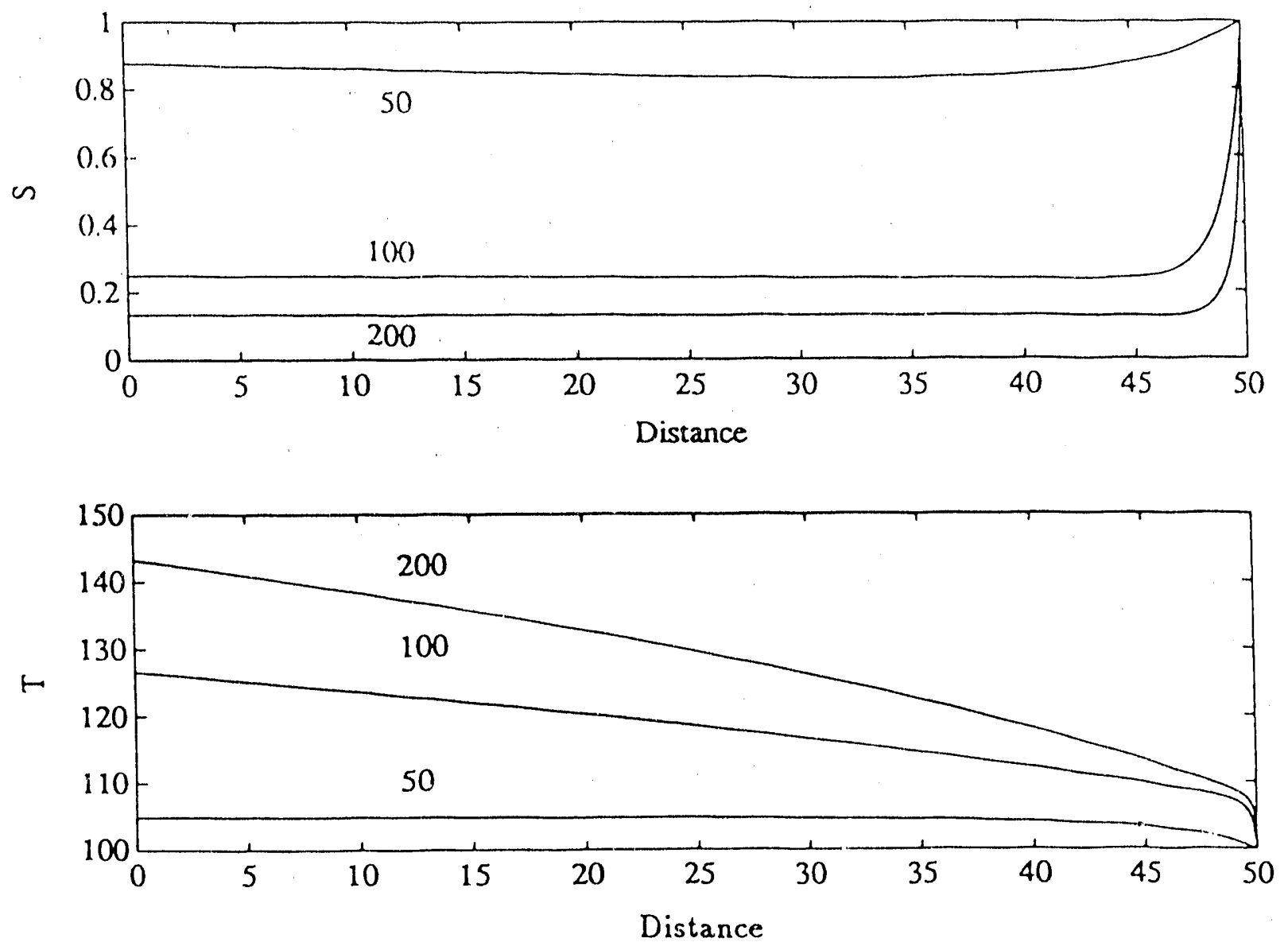

Figure 34: Effect of energy injection rate $C(\mathrm{btu} / \mathrm{hr})$ on saturation and temperature profiles. 
,$\| R_{1}$.

For completeness, we also present saturation and temperature profiles corresponding to a heterogeneity (permeability) field that obeys fractal statistics (fractional Brownian motion, fBm). The relevance of $\mathrm{fBm}$ statistics to field heterogeneity was conjectured by [36]. Results for $H=0.7$, corr'sponding to a fractal dimension $D=1.3$ ([28]), are shown in Figure 36. The permeability field having long-range correlations $(I>0.5)$, permeability gradients are generally small, thus the saturation response is relatively mild (although sharp responses are expected for negatively correlated fields, $I I<0.5$, see $[104])$. Even smaller is the effect on the temperature profile.

In the above, rate conditions were selected such that flat saturation profiles were likely to develop over most of the core extent (see also related discussion below). Contrary to non-condensing flows, however, flat saturation profiles in vapor-liquid flows cannot be extended indefinitely. Beyond a cretain interval, liquid saturations start to slowly increase in the upstream direction, and conditions of single-phase liquid flow eventually set. From the conventional forward direction, these processes correspond to the in-situ change of phase descrihed by [20], [32] and [54]. To obtain useful information about such flows the corresponding saturation-temperature trajectories must be examinol.

\subsection{SATURATION-TEMPERATURE TRAJECTORIES}

firr the remainder, homogeneous cores only will be considered. Here, saturation and temperature "Ir" uniquely related, as can be seen by eliminating from (47)-(48) the space variable. The soIntions of the resulting equation constitute trajectories in the "phase (composition) space," each corresponding to a specific initial (outlet end) condition. The display (phase portrait) of such trafrotories is of interest, as it allows for qualitative conclusions to be reached. Since our main interest is in two-phase flow, initial conditions at the outflow end will be specified, by taking variable temfirratures and either $S=1$ or $S=0$. This particular selection of saturation values is necessitated hy and afferts (saturation, slightly subcooled or slightly superheated conditions, respectively). The precise specification requires precise knowledge of the exit conditions, a rather difficult task in practical applications. We shall return to this issue below.

fir converience, we rewrite the trajectory equation to be solved in the form 

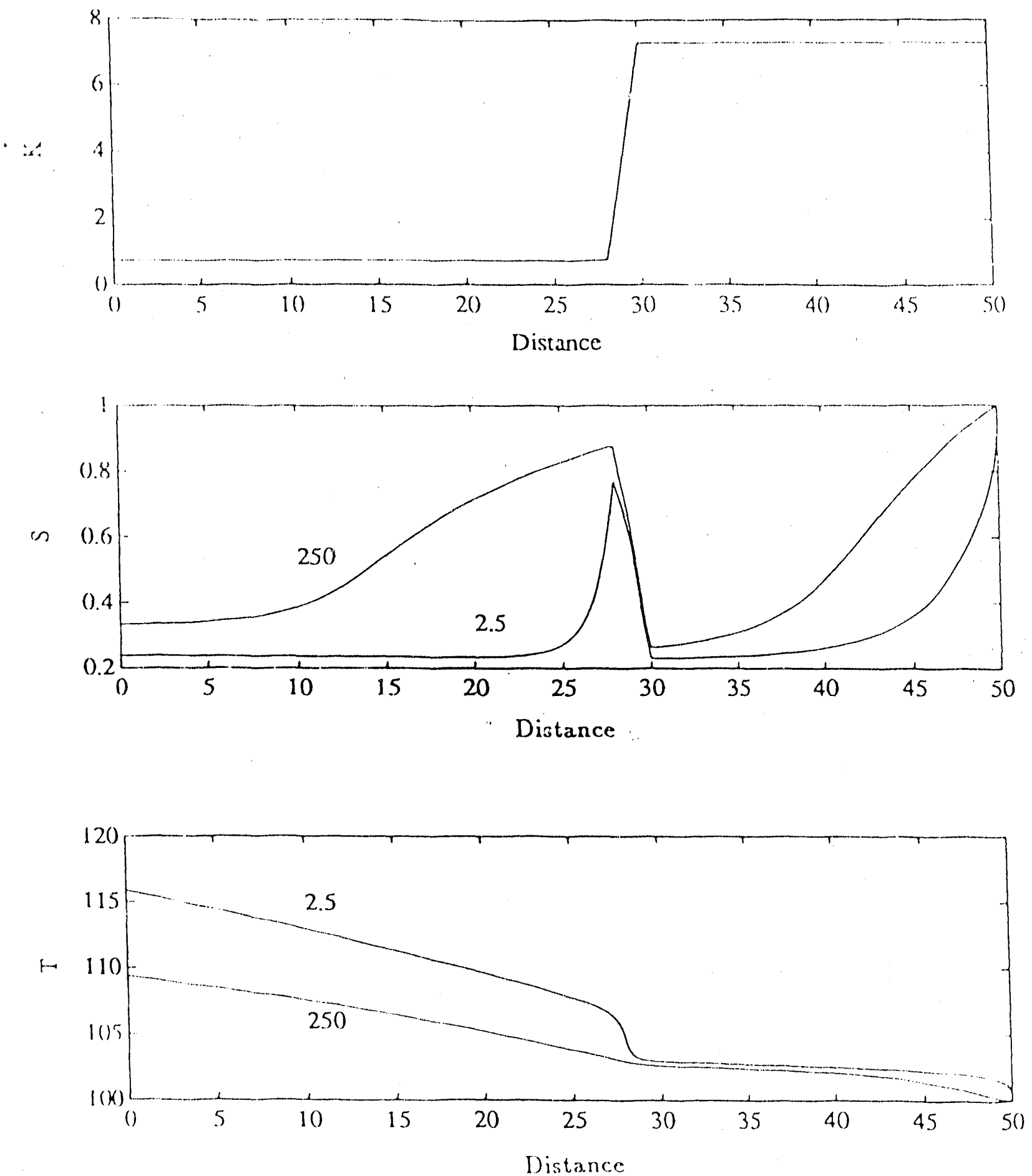

Figure 35: Capillary heterogeneity: effect of conductivity for a ste $e_{1}$ increase in permeability $(\lambda$ in $\left.H / m^{\prime \prime} K\right)$. 

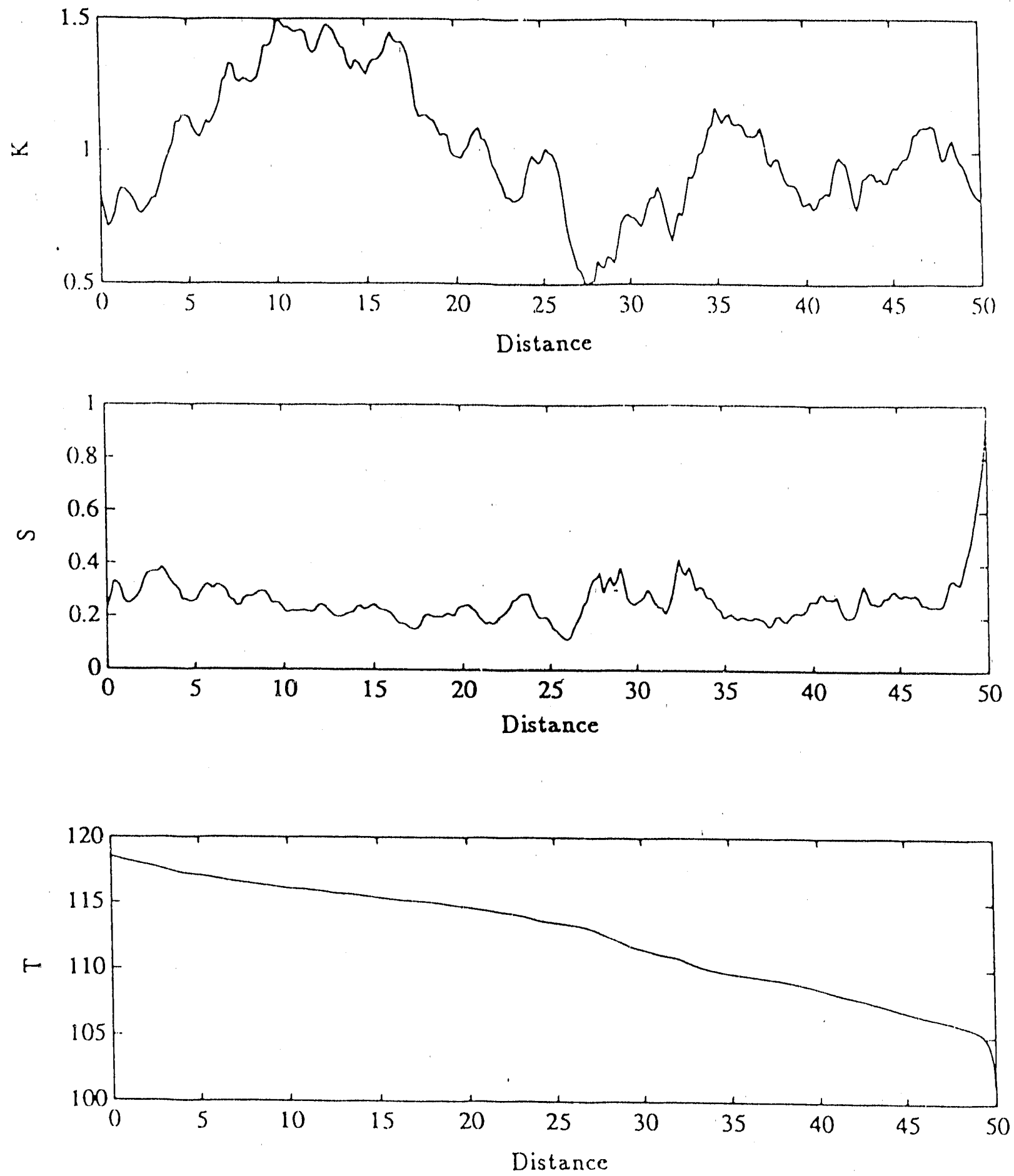

Figure 36: Capillary heterogeneity: saturation and temperature response for an fbra permeability profile $(H=0.7)$. 


$$
\frac{d J}{d S} \frac{d S}{d \Theta}=\frac{F(\Theta, S)}{G(\Theta, S)}
$$

where, in the absence of heterogeneity

$$
\begin{aligned}
F(\Theta, S) \equiv & \frac{K R_{p} A}{\Theta^{2}}\left(W-\frac{E}{R_{m}}\right) k_{r L}+ \\
& \frac{K R_{p} A}{\Theta^{2}}\left(-\frac{E}{R_{m}}\right) R_{\mu} R_{p} k_{r v}+R_{h} \\
G(\Theta, S) \equiv & \left(1+b R_{p} A\right)\left(W-\frac{E}{R_{m}}\right) k_{r L}+ \\
& b R_{p} A\left(-\frac{E}{R_{m}}\right) R_{\mu} R_{p} k_{r v}
\end{aligned}
$$

For negligible Kelvin effects we may further approximate

$$
G(\Theta, S) \simeq\left(W-\frac{E}{R_{\dot{m}}}\right) k_{r L}
$$

Expressions for the heat input rates $W-\left(E / R_{m}\right)$ or $-E / R_{m}$ are given in the Appendix.

An important element of the phase portrait is the critical curve $F(\Theta, S)=0$ (curve $A$ in Figure 37 ), which controls the sigu of the saturation slope $d S / d x$. Of less importance is the other critical curve $G(\Theta, S)=0$ (curve $B$ in Figure 37), which controls the sign of the temperature slope $d T / d x$. The two curves divide the $(\Theta, S)$ plane into three regions: Trajectories in regions I and III have negative slope that depends mainly on $R_{h}$, while those in II have positive slope. The dividing curves $A$ and $B$ approach each other near $S=1$, at which point ( $D$ in Figure 37), for $R_{h} \ll 1$, the corresponding temperature, $T_{2}$, is the solution of

$$
h_{L}\left(T_{2}\right)=\frac{C}{m_{i}}
$$

This is the temperature at which the saturated liquid has the enthalpy of the injected fluid. We note that for other parameters kept fixed, the phase portrait is a function of the injected energy per unit mass, $C / m_{i}$. An increase in the latter leads to an enlargement of region I, towards higher temperatures and lower saturations. 


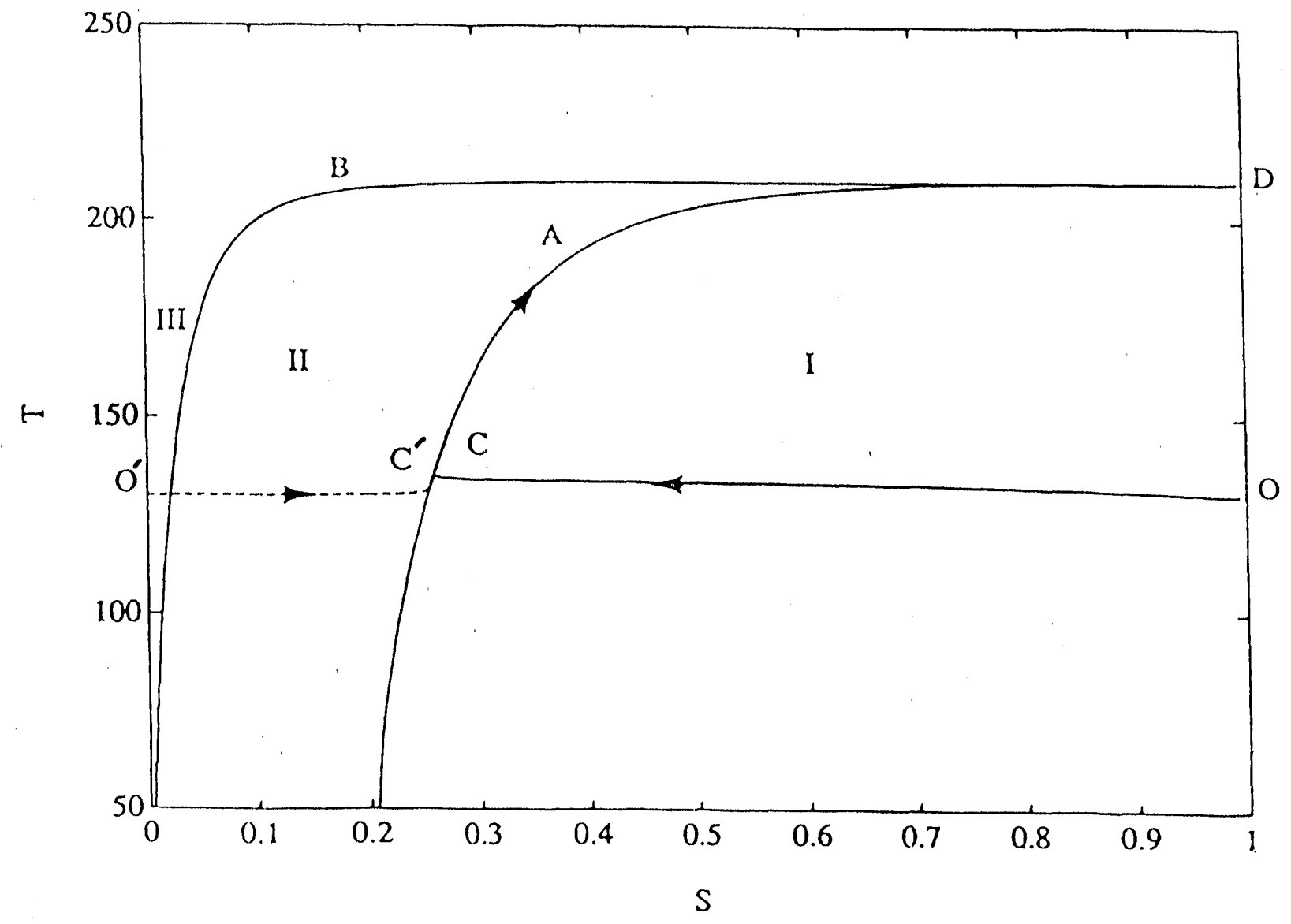

Figure 37: Phase portrait and $(T, S)$ trajectories schematic. Arrow indicates upstream direction. 
It is of interest to follow a typical trajectory. We consider, first, trajectories starting from the axis $S=1$ (point $O$ ). While in region $\mathrm{I}$, the trajectory has a (slightly) negative slope, reflecting the small temperature increase as the saturation decreases in the upstream direction (indicated by the arrow). This slope can be substantial when parameter $R_{h}$ is large (e.g. small $k$ or large $\lambda$ ). It is in this region that capillary end effects are significant, and in fact, region $I$ is essentially a magnification of the boundary region near the outlet end (Figure 34). One should note that the saturation spatial profile does not become flat until the critical curve $A$ is encountered.

Upon approach of curve $A$, the function $F$, thus the saturation slope, become very small, and the corresponding saturation profile is nearly flat. This is also reflected in the direct integration of the previous set (47)-(48) in this region which is extremely slow, and has, in fact, prompted us into using (18) as an alternative to direct integration. Subsequently, the trajectory crosses over $A$ at the turning point ( $C$ in Figure 37), where $F$ changes sign, region II is entered, and the trajectory changes direction and acquires a positive slope. Curve $A$ acts now as an attractor, the solution trajectory becoming almost indistinguishable from it to the printer's resolution. Both saturation and temperature increase in the direction of the arrow (upstream), until single-phase flow conditions are approached (point $D$ ). In this region, all trajectories, regardless of their initial (outlet) condition practically coincide with curve $A$, implying that all in-situ vaporization regimes lie on the same curve for constant enthalpy content $C / m_{i}$.

Similar results are obtained for solution trajectories that emanate from the axis $S=0$, instead (point $O^{\prime}$ in Figure 37). Such trajectories have a slightly negative slope in region III, while being practically flat in most of region II. As in the previous, however, flat saturation spatial profiles are encountered only when the critical curve $A$ is approached $\left(C^{\prime}\right)$. Thereafter, the trajectory becomes attracted to $A$, which it closely follows until the single-phase point $D$. In fact, the two different trajectories (whether starting from the "liquid" or the "vapor" side) coincide for all practical purposes away from the boundaries. We should mention that similar effects were also observed in the related study of countercurrent vapor-liquid flow ([81]).

Before proceeding further, several remarks are appropriate. We, first, note that such phase portraits unify the different two-phase flow regimes that develop in the two kinds of experiments, e.g. the [79] or the [54] type. The former case (most appropriate for relative permeability measurements) corresponds to the segments $C O$ (or $C^{\prime} O^{\prime}$ ) where capillary end effects can be important. Clearly, 
starting (injection) points can be anywhere along the trajectory, and they are determined by the rate. parameter, $R_{m}$ (see also below). In the absence of additional information the precise path selection $\left(C^{\prime}()\right.$ vs. $\left.C^{\prime} O^{\prime}\right)$ may be unclear. However, the (slightly) negative slope in region 111 implies a t.mperature increase in the downstream direction near the outlet end, for trajectories cmanating from $S=0$. This leads us to tentatively conjecture against the existence of such solutions at. steady-state. However, additional work is needed to conclusively resolve this issue.

The other case involves the entire trajectory $(D C O)$ and corresponds to evaporation in situ.

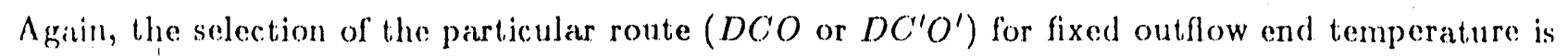
uncertain. Ilowever, the practical consequences of this uncertainty are not significant in the present. case, since the relative length of the last segment $\left(C O\right.$ or $C^{\prime} O^{\prime}$ vs. $D C O$ or $\left.D C^{\prime} O^{\prime}\right)$ is negligible. Because of this, experiments of this type are likely to offer useful information only as it regards saturations in the main path $D C$ (or $D C^{\prime}$ ).

The physical meaning of curve $A$ is the condition of constant enthalpy flow in the absence of capillarity (relatively high values in $R_{p}$ ). This is most readily seen in the no-conduction limit, where in dimensional notation one obtains

$$
\frac{k_{r L}}{R_{\mu} R_{p} k_{r v}}=-\frac{h_{v}(T)-\left(C / m_{i}\right)}{h_{L}(T)-\left(C / m_{i}\right)}
$$

By rearrangement, it is easily shown that (59) indeed expresses the adiabatic condition of constant enthalpy in the absence of capillary and heat conduction effects. We note that at such conditions, this is essentially the solution of Miller in his original paper (1951). In fact, the entire curve $A$ can be readily transformed into the "pressure function" considered by [54] by a simple change of variables. By further neglecting the variation of latent heat with temperature, the critical curve becomes vertical (constant. $S^{\prime}$ )

$$
f_{W}(S)=1-\chi_{0}
$$

and where $\lambda_{0}$ is the injected vapor quality (compare with previous section). Temperature and heat conduction effects canse the notable departure from the vertical.

I'rajectories for water and propane for the conditions of Tables 3 and 4 are shown in Figures 38 and 39, respectively. The attraction, of the trajectories to the single critical curve is evident in both figures. This feature is a consequence of negligible capillarity compared to the saturation pressure, 
particularly at higher temperatures. Parameter $R_{p}$ measures this effect at reference conditions. For the case of propane at conditions of Table 4, the large value of $R_{p}$ and the high permeability (low $R_{h}$ ) verify the correctness of the approach used by [54].

This need not be the case, however, for lower permeability (or higher conductivity) cores. The phase portrait in Figure 40 corresponds to a 100 -fold reduction in permeability and shows several significant differences: The size of region I has been reduced, the critical curve $A$ no longer coincides with the limiting curve in the abence of conduction (e.g. Figure 37), while the trajectories in region I take a significant slope, indicating the added importance of capillarity at lower permeabilities. Nevertheless, at sufficiently large temperature (pressure) all trajectories get eventually attracted to the single curve $A$ (compare Figures 38 and 40 ). Additional permeability effects are presented below.

\subsection{PARAMETER SENSITIVITY}

The preceding section showed that for a fixed enthalpy per unit mass the phase portrait is fixed. The particular solution trajectory was then determined by the exit temperature (pressure). The spatial saturation and temperature profiles, however, depend additionally on the rate parameter $R_{m}$. This is the only length-dependent parameter. The latter determines whether evaporation insitu ([54]) or flat saturation profiles ([78]) or an intermediate regime would be established. The three possibilities are depicted in Figure 41. We point out that the saturation-temperature pairs in each of the three regimes of Figure 41, all belong to the same trajectory, each experiment representing a magnification of a region of the previous one.

Conditions for in-situ evaporation can be precisely specified. For example, the minimum length of the core required follows from the requirement that

$$
R_{m} \geq X_{2}
$$

where $X_{2}$ depends on the energy content $C / m_{i}$ and on the exit temperature $\left(\Theta_{1}\right.$ in dimensionless notation), and it is given from (48)

$$
X_{2}=-\int_{\Theta_{1}}^{\Theta_{2}} \frac{H\left(\Theta, S\left(\Theta ; \Theta_{1}\right)\right)}{G\left(\Theta, S\left(\Theta ; \Theta_{1}\right)\right)} d \Theta
$$




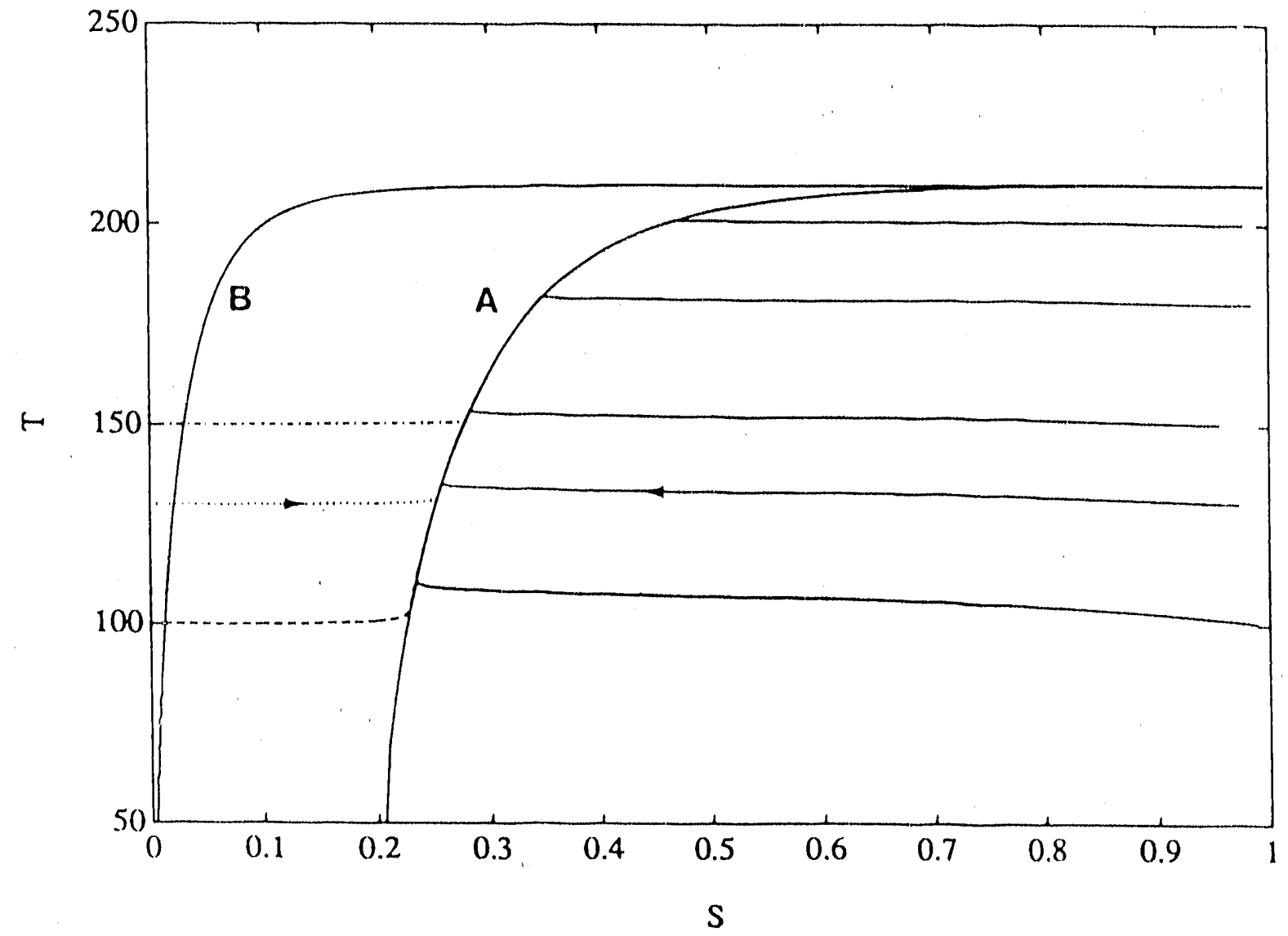

Figure 38: Phase portrait and $(T, S)$ trajectories for water at conditions of Table 3. Arrow indicates upstream direction. 


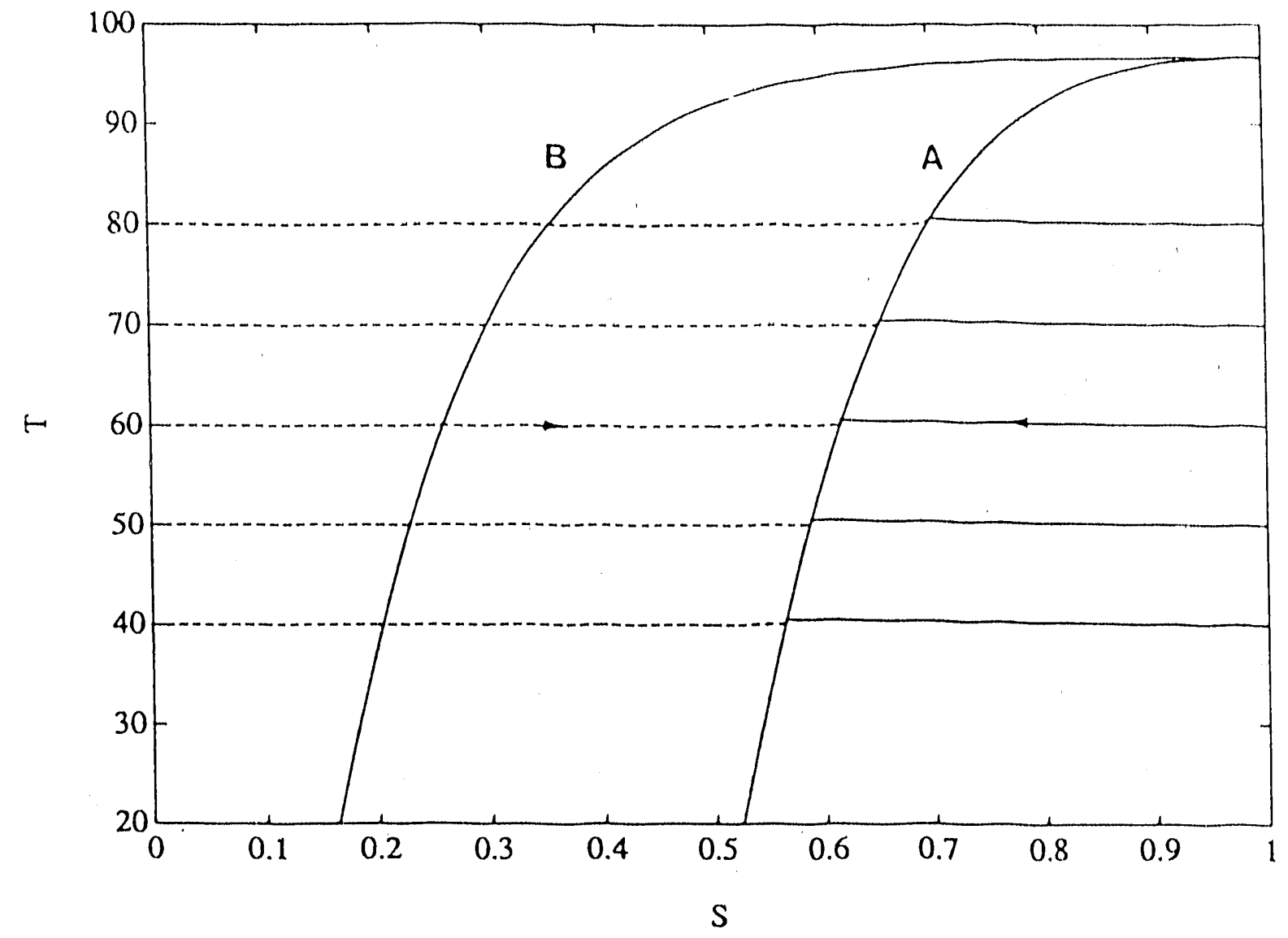

Figure 39: Phase portrait and $(T, S)$ trajectories for propane at conditions of Miller (1951). Arrow indicates upstream direction. 


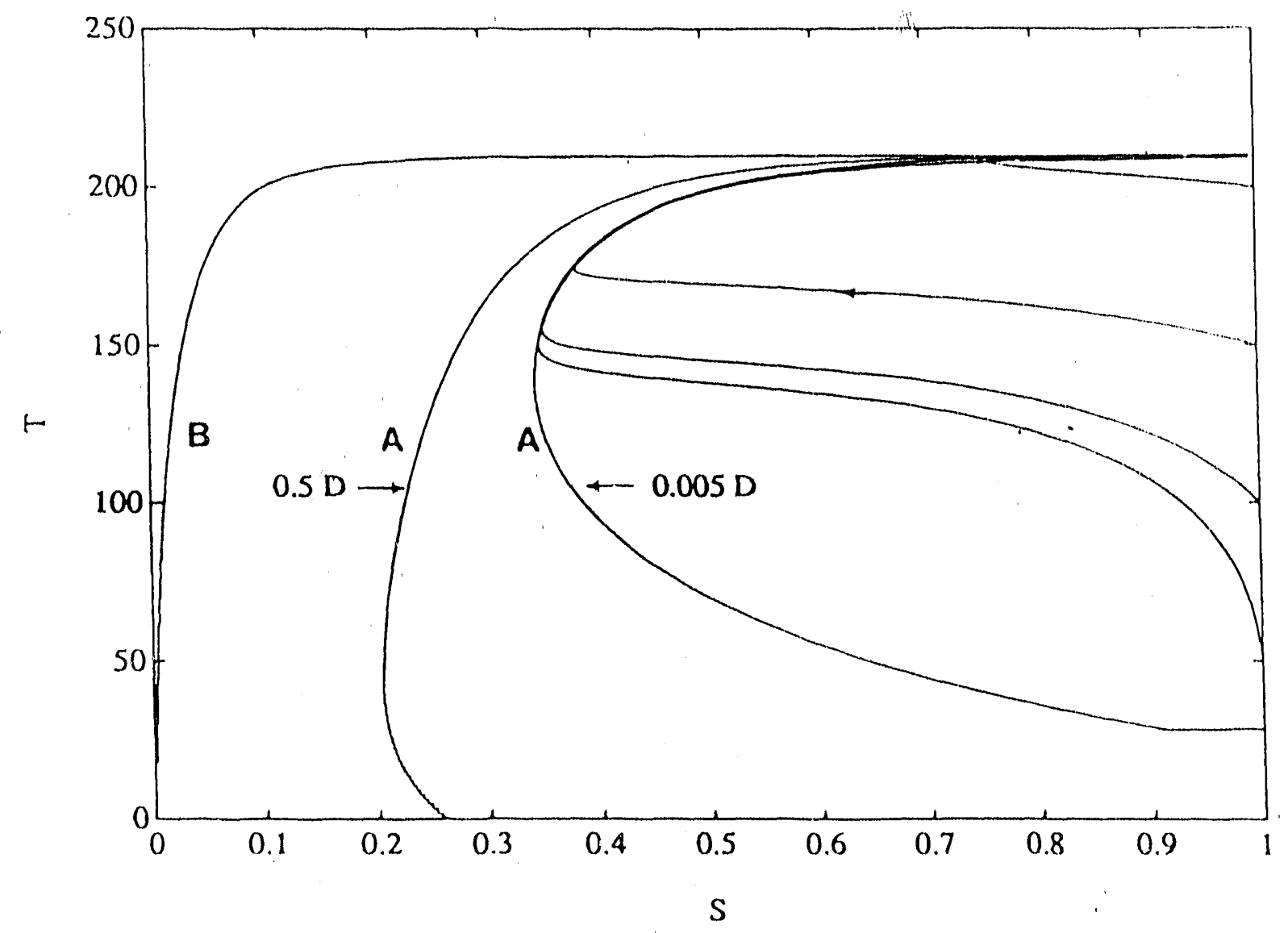

Figure 40: Phase portrait and $(T, S)$ trajectories for water at conditions of Table 3 . Effect of permeability. Arrow indicates upstream direction. 

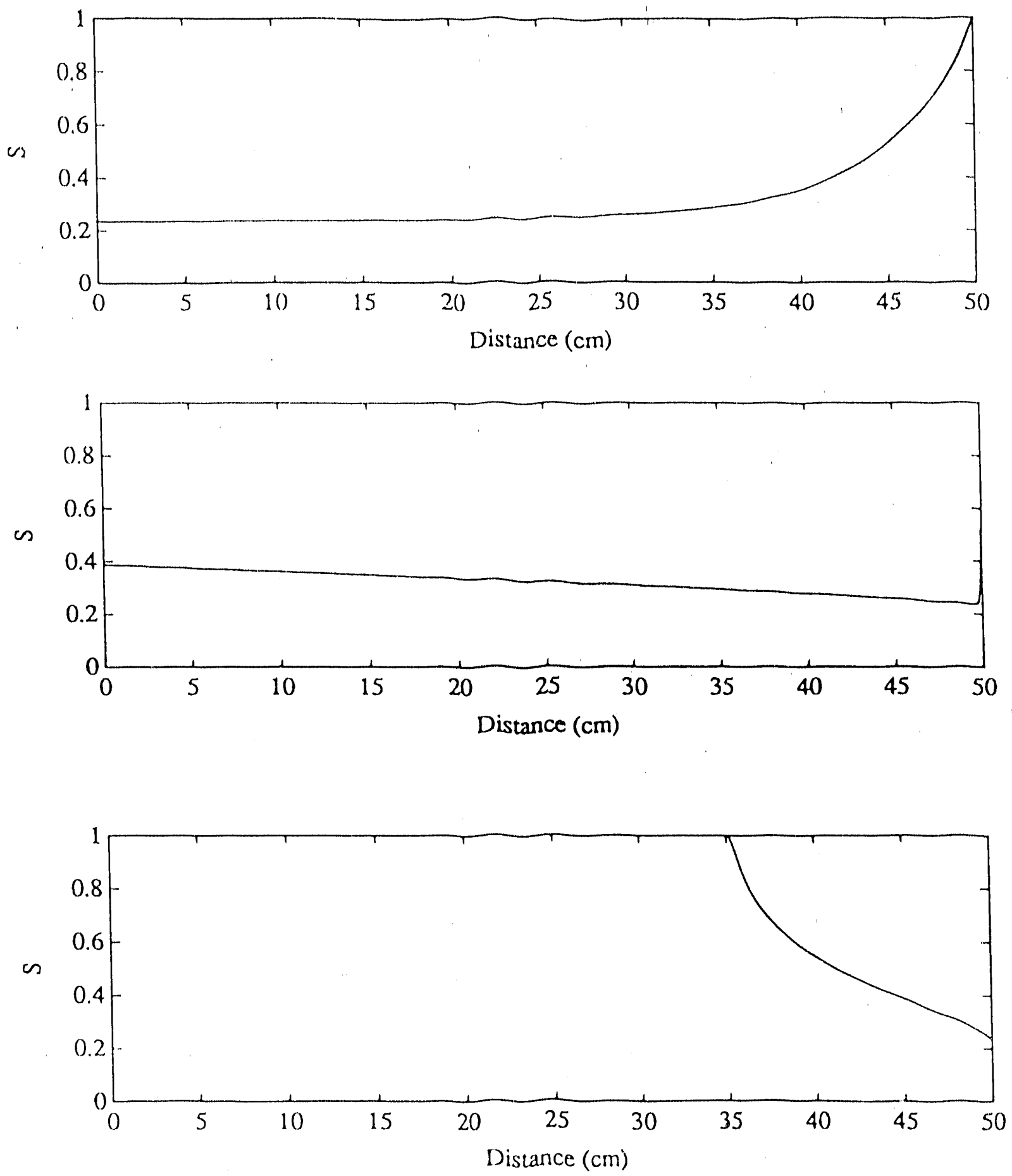

Figure 41: Sensitivity of saturation profiles to the rate parameter $R_{m}\left(R_{m}=0.01,1,10\right.$ from top to bottom). 
The above integral is evaluated along the particular trajectory, emanating from $\Theta_{1}$, with $S$ solving (53).

The variation of $X_{2}$ with the outlet temperature was investigated for the conditions of experiment $\mathrm{V}$ by [54] shown in Table 4. In general, $X_{2}$ was found to decrease as $T_{1}$ increases and as the thermal energy content $C / m_{i}$ decreases. Sample values obtained were 45.7 and 19.2 for $T_{1}$ equal to $40^{\circ} \mathrm{F}$ and $80^{\circ} \mathrm{F}$, respectively. Comparison with Table 4 shows readily that condition (61) is indeed satisfied. It was previously pointed out that experiments of this type sample regions of saturation higher than the value corresponding to the turning point, $S^{*}$. As one may expect, end effects are insignificant for in-situ evaporation. A quantitative assessment is readily obtained by comparing $X_{2}$ to $X^{*}$, where $X^{*}$ is evaluated from (62) with $\Theta^{*}$ as the upper limit of integration. For the conditions of Table 4, one obtains $X^{\star}=0.15$, which is negligibly small in comparison to $X_{2}$.

When condition (61) is not satisfied, two-phase flow occurs throughout the core. Given the exit temperature (pressure), the specific trajectory is fixed, thus saturation and temperature profiles can be determined. Inlet conditiona $\left(S_{0}, T_{0}\right)$ are obtained by solving the equation

$$
R_{m}=-\int_{\Theta_{1}}^{\Theta_{0}} \frac{H\left(\Theta, S\left(\Theta ; \Theta_{1}\right)\right)}{G\left(\Theta, S\left(\Theta ; \Theta_{1}\right)\right)} d \Theta
$$

and subsequently evaluating $S_{0}$ with the use of the trajectory equation (53). The integration of equation (48), rather than the saturation equation (47) must be noted. Contrary to the saturation, which exhibits a turning point, the temperature is monotonically varying, thus facilitating the integration. This aspect is particular to two-phase, single-component systems.

Typical results are shown in Figure 43, for various values of the energy content and the conditions of Table 3. As $R_{m}$ increases (by increasing the total rate or the system length), $S_{0}$ decreases until the turning point value $S^{*}$ is reached. A significant plateau region develops thereafter, the extent of which increases with the energy content (steam quality) and the permeability. In view of the various uncertainties involved in the estimation of parameters, the existence of such a plateau over orders of magnitude in $R_{m}$ is highly desirable. Unfortunately, its size decreases significantly at lower values of $C / m_{i}$ and at lower permeabilities (see below). This sensitivity is somewhat similar to that of injected quality $\chi_{0}^{\prime}$, exhibited in more simplistic approaches, where $S^{*}$ is taken to be the solution of (60). A plot of this approximation to $S^{*}$ is shown in Figure 42. The sensitivity near low values of the injected quality is quite notable and it has been conjectured to cause errors in the 
estimation of relative permeabilities in that region.

The success of most relative permeability experiments relies on the condition that a flat profile be established throughout the core with $S^{*}$ as the core inlet saturation. To ascertain this, the relative importance of end effects must be considered. As a measure of the deviation from flat profiles we consider the following parameter

$$
\delta=\int_{0}^{1}\left(\frac{S-S^{*}}{S^{*}}\right) d \xi
$$

Upon rearrangement and use of (47), (48) and (53), this becomes

$$
\delta=-\frac{1}{R_{m}} \int_{\Theta_{1}}^{\Theta_{0}}\left(\frac{S}{S^{*}}-1\right) \frac{H\left(\Theta, S\left(\Theta ; \Theta_{1}\right)\right)}{G\left(\Theta, S\left(\Theta ; \Theta_{1}\right)\right)} d \Theta
$$

Since $\Theta_{0}$ is implicitly related to $R_{m}$ via (63), equation (65) gives $\delta$ as a function of $R_{m}$, other parameter kept constant.

Plotted in Figure 44 is the error estimate $\delta$ as a function of the rate parameter $R_{m}$, for the conditions of Figure 43. Roughly paralleling the inlet saturation plot (Figure 43) the error decreases considerably as $R_{m}$ increases, particularly after the turning po'nt has been exceeded. More importantly, a plateau region of small error values develop, the extent of which can be considerable for high $C$ and $k$. This is highly desirable, since large variations in $R_{m}$, the proper values of which cannot be a priori known, only result in relatively small errors. Upon leaving the plateau region, however, substantial errors are rapidly estabiished, that prohibit the successful conduct of meaningful relative permeability experiments there

The error estimate was fo and to be sensitive to the injected energy content, more reliable results being obtanned at higher injection quality, roughly in step with the saturation sensitivity (Figures 43 and 44). In general, the plateau extent decreases and errors increase with a decrease in the injected energy content, although the minimum error value is not as :ensitive and remains low, at least for the cases studied. On the other hand, errors are uniformly higher for lower permeability cores. Plotted in Figure 45 are the inlet saturation and the corresponding error estimate for the conditions of Table 3, but with a hundred-fold reduction in permeability. It is apparent that the plateau disappears, and that only a narrow region remains, where, however, errors are sign ficantly higher than befure (the luwesi value being higher than $33 \%$ in the case of Figure 45 ). It is clear 


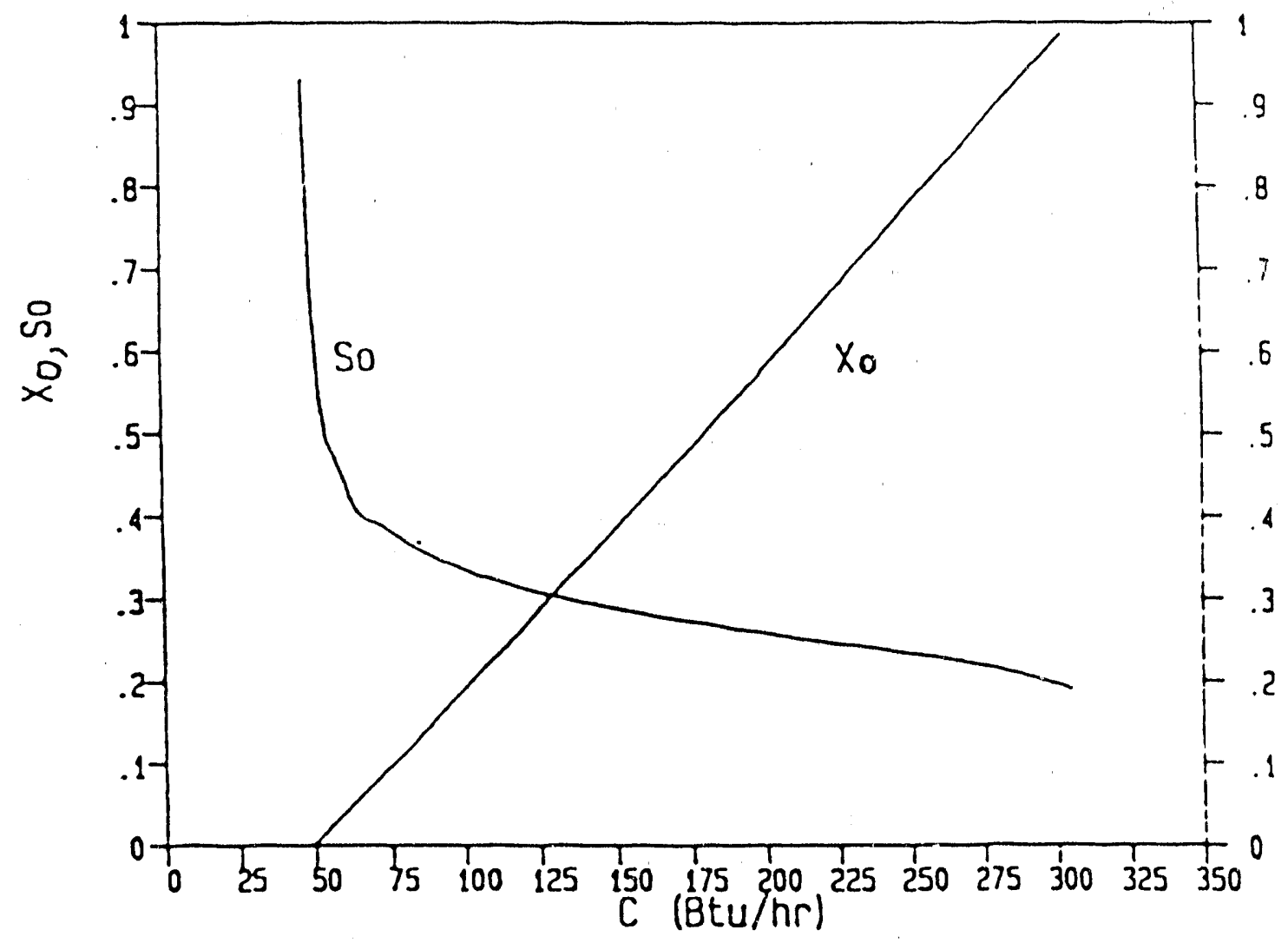

Figure 42: Sensitivity of inlet saturation to steam quality and heat injection rate for the conditions of Sanchez and Schechter (1987). 


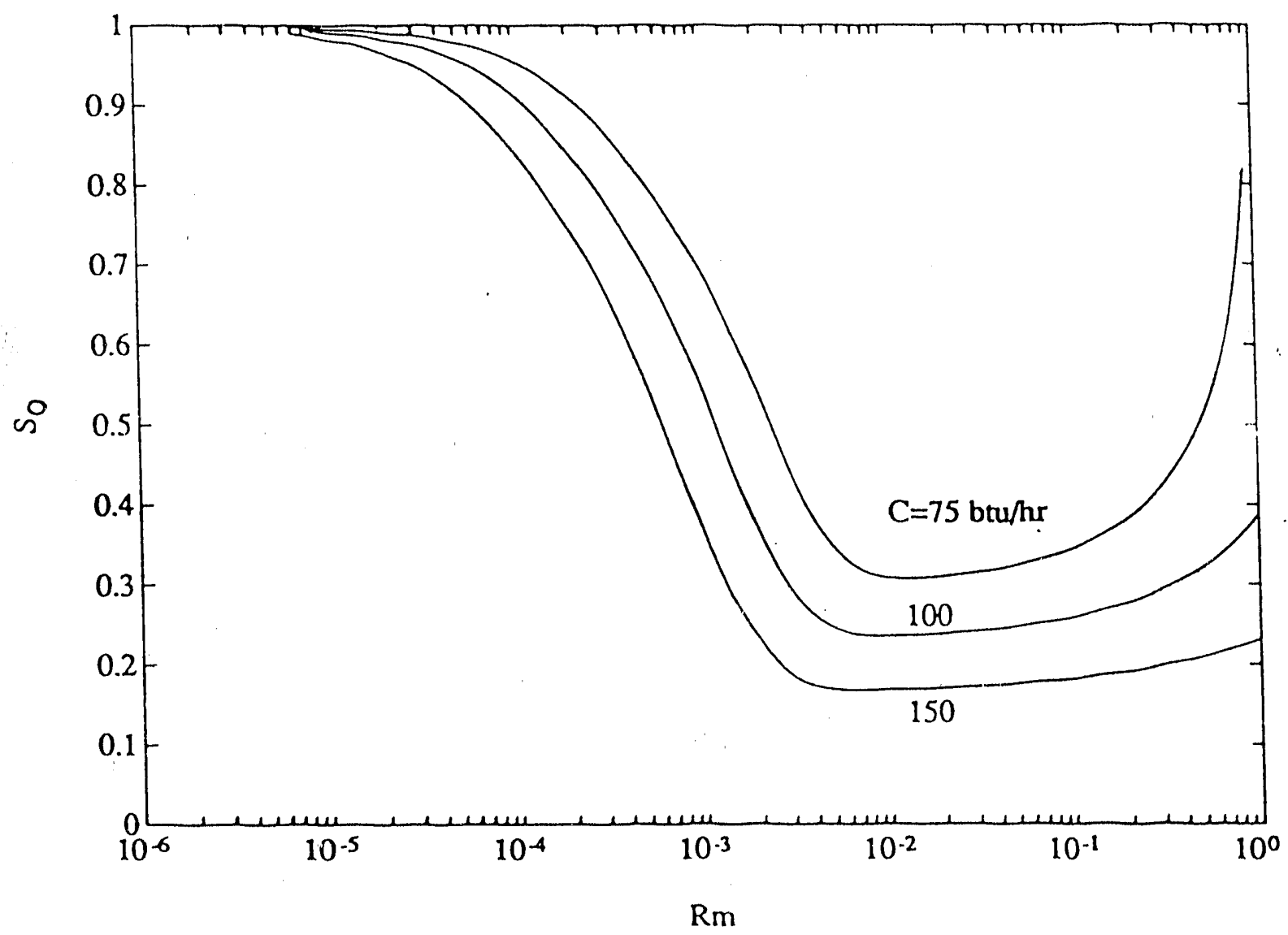

Figure 43: Sensitivity of inlet saturation $\left(S_{0}\right)$ to the rate parameter $R_{m}$ for standard conditions: Effect of heat injection rate $C(\mathrm{btu} / \mathrm{hr})$. 


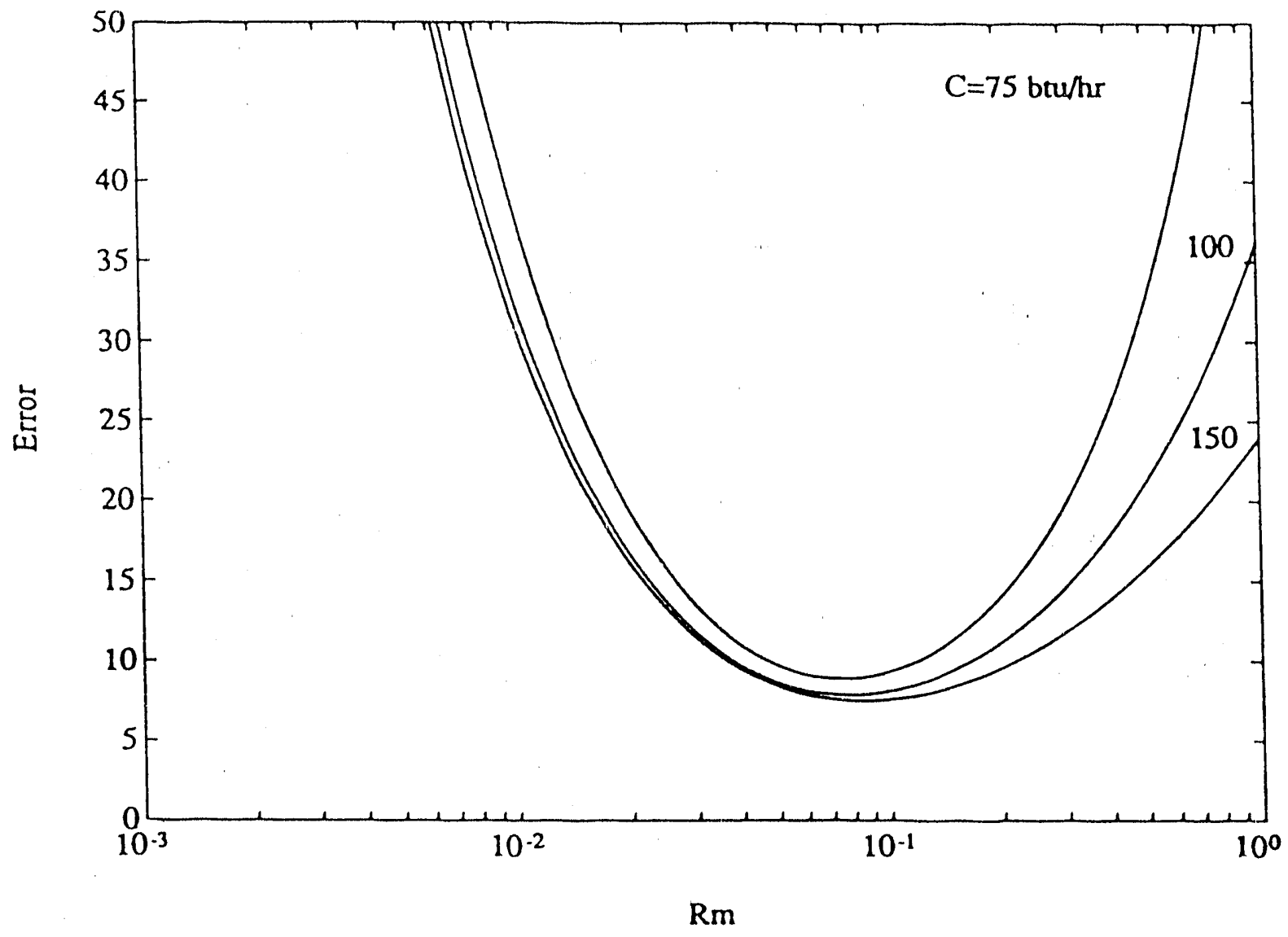

Figure 44: Sensitivity of error estimate to the rate parameter $R_{m}$ for standard conditions: Effect of heat injection rate $C(\mathrm{btu} / \mathrm{hr})$. 
that meaningful relative permeability experiments of this type are not possible for such cases, and alternatives must be sought.

As a sample illustration of the above sensitivity we simulated the error involved in one of the experiments (run no.15) reported by [78]. Except for the capillary pressure curve (for which the expression of Table 2 was used), all other parameters including relative permeabilities were reported. The corresponding inlet saturation and error estimate plots are shown in Figure 46. Notable are the wide plateau regions and the corresponding low error values, both resulting from the large core permeability. Also marked in Figure 46 are the inlet saturation $\left(S_{0}=0.2927\right)$ and error $(\delta=7.7 \%)$ estimates corresponding to the experimental value for this run, $R_{m}=0.015$. The relatively low error estimate, coupled with the good agreement between theoretical and experimental $\left(S_{0}:=0.31\right)$ values underscores the reliability of these data (although an analysis of all the runs is of course necessary for a more rigorous assessment).

\subsection{CONCLUDING REMARKS}

In this chapter we analyzed steady-state, concurrent vapor-liquid flows in 1-D cores under adiabatic conditions. The general problem was treated including heat conduction, capillarity, phase change and Kelvin effects. The latter were found to be negligible, except at the low liquid saturation range, where, however, unaccounted thin film effects become quite important and must be included. The aim of the study was to examine the features of saturation and temperature profiles, to explore their sensitivity to process parameters, and to provide error estim.ztes for relative permeability vapor-liquid experiments typically conducted at steady-state.

It was, first, shown that reliable numerical results are only possible by backwards (in the upstream direction) integration, a requirement that can be met only if (outlet-) end conditions are available. This led us to the analysis of end effects and, in general, of effects caused by capillary heterogeneity. For small values of the conduction parameter $R_{h}$ (small conductivity or large permeability), the effects on the liquid (wetting) saturation are identical to the case of two-phase, two-component (oil-water) flow, analyzed in detail in a recent work ([104]). The temperature profile is also affected, becoming steeper (smoother) near the heterogeneity region for a downstream increase (decrease) in the permeability. Direct extrapolation to conventional end effects suggests a mañinum liquid saturation buildup, accompanied by condensation and sharp temperature gradi- 

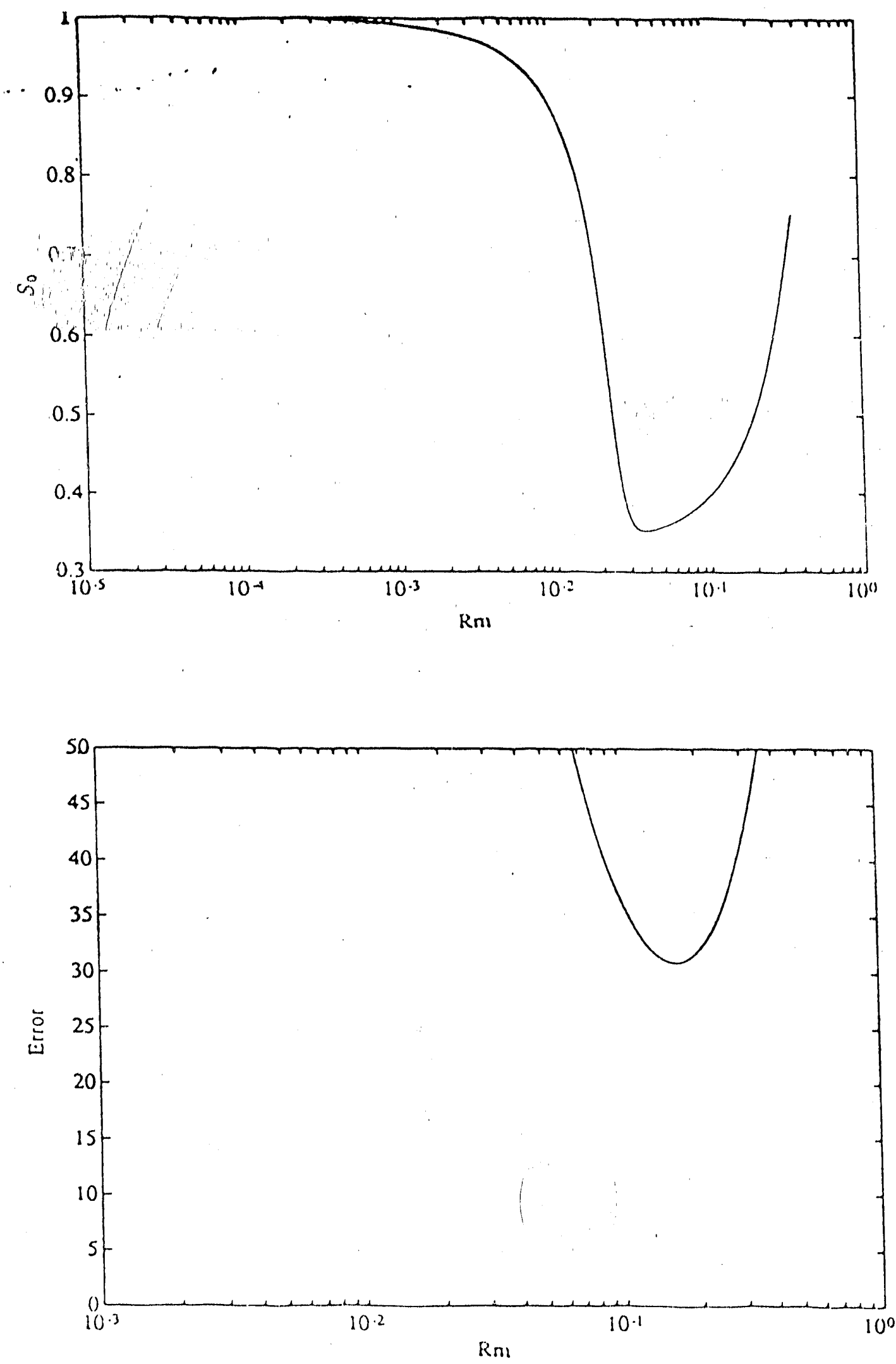

Figure 45: Sensitivity of inlet saturation and error estimate to the rate parameter $R_{m}$ for standard conditions and permeability of $0.005 d$. 

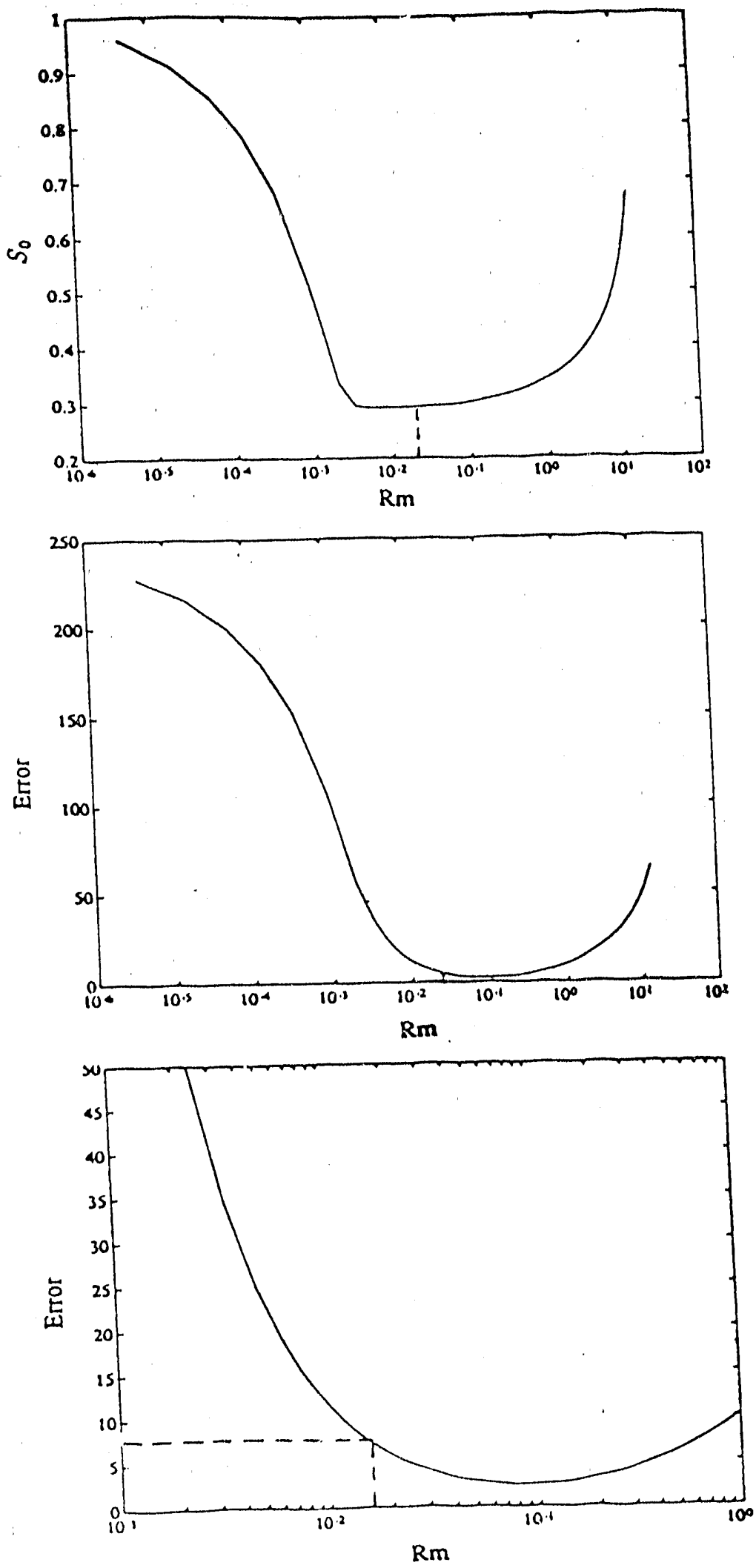

Figure 46: Sensitivity of inlet saturation and error estimate (at two different magnifications) to the rate parameter $R_{m}$ for conditions of Run 15 in Sanchez and Schechter (1987). 
ents at the outlet end. Opposite effects are to be expected at the inlet (although such extrapolation may not be strictly proper). Conductivity was found to exert significant effects by extending the influence of the heterogeneity to a greater region. While unimportant for large permeability cores, such effects are quantitative for tight cores.

Subsequently, the flow in homogeneous cores was simulated in terms of temperature-saturation (composition) trajectories. This approach enables a unified approach to the vapor-liquid flow that encompasses both the in-situ vaporization of [54] (injection of a subcooled liquid) and the simultaneous injection of two-phases, as is typical in relative permeability experiments ([79]). It was found that all solution trajectories are rapidly attracted (away from end effects) to the critical curve that describes flow in the absence of capillarity. It is in its near vicinity, where inlet conditions in typical relative permeability experiments also lie. Under the additional constraint of negligible conduction, this curve coincides with the solution of [54]. However, significant discrepancies arise as the permeability decreases.

Based on the above, parameter sensitivity studies were conducted. Quantitative requirements on the rate parameter $R_{m}$ were obtained for in-situ vaporization to occur. Typical minimum estimates range between 10 and 50 for the cases studied. The sensitivity of the inlet saturation (for fixed outlet temperature) was next considered. It was shown that a plateau-like region develops covering a range of $R_{m}$ that may span several orders of magnitude, depending on the permeability primarily, and on the injected energy content, to a lesser extent. Such plateau regions are desirable, in order to suppress parameter sensitivity and related errors. An error measure was also defined and found to obey a similar dependence. The existence at high permeability values $\left(R_{h} \ll 1\right)$ of a wide region of low errors indicates that relative permeability estimates obtained at such conditions can be reliable. A sample illustration using data from [79] provides supporting evidence. On the other hand, serious questions should be raised regarding the applicability of current methods for vapor-liquid relative permeabilities of tight cores. It was shown that the lowest possible errors can be quite large, while being very sensitive to the rate parameter. We conclude that for such cases alternative methods are needed. 


\title{
5 NUMERICAL SYNTHESIS AND SIMULATION OF FRACTURED SYSTEMS USING FRACTALS
}

\author{
J.A. Acuna and Y.C. Yortsos
}

\subsection{INTRODUCTION}

A fractal image is basically a repetition of a given geometric shape into itself over a cascade of different length scales. The apparent complexity that results from such iteration, which is often coupler. with random noise, has made fractal images suitable for the description of natural objects. Although this should not imply that every object in nature is fractal, nonetheless fractals constitute a convenient way to describe both the process and the result of physical reality. Special cases are objects that offer little hope for a conventional Euclidean geometry description such as ferns and clouds.

The application of fractals to porous media in general is very promising. The review by Sahimi and Yortsos [76] classifies the fractal patterns resulting from various processes such as percolation, viscous fingering and fracturing. Networks of natural fractures in a rock are also candidates for a fractal geometry description. This alternative is explored in this Chapter.

Previous efforts in this direction are relatively recent. In 1985, Barton [7] started an investigation to apply fractal concepts for the description of naturally fractured systems. His work consisted of mapping and studying the fractal properties of the fracture network at Yucca Mountain, NV. and concluded that many mapped fracture patterns were self-repetitive over a range of scales that span from 0.2 to 10 meters. He also reported the existence of sevcral generations of fractures, distinguishable from each other by their position and by their relation with respect to other fractures. His promeering work is mostly descriptive and, to our knowledge, the observed fractured systems were not reproduced numerically. Additional field evidence for the fractal nature of fracture networks, aithough, at present only qualitative, is provided from the visual inspection of fracture patterns, such as those of the Monterey formation [30]. Prominent fractal features in the latter include the cascade of scales of fractures and the underlying self-similarity. Theoretical support for the fractal nature of fracturing can be found in the work of Sammis et al. [78].

Fractal geometry appears to have great potential for the characterization of fracture networks. At this stage, our work is aimed at the generatior of synthetic fracture networks that incorporate 
fractal concepts and, thus, represent adequate images of fracture networks. Within this network, fluid flow and transport are to be simulated. The application of the model images to the interpretation of data from real naturally fractured reservoirs and process simulation constitute the ultimate goals of this investigation. In this chapter, a numerical method for their construction and a preliminary investigation of fluid transport are presented.

\subsection{GENERATION OF FRACTAL FRACTURE NETWORKS}

Barnsley [6] has proposed a method to construct fractal images, using the Iterarive Function System (IFS). Under this technique, a fractal image is constructed by applying to an initial figure a set of numerical transformations in an iterative fashion. If $n$ equations compose the system, each new iteration creates multiple sets of $n$ smaller, transformed images that occupy the place of the previous image. Every new generation is successively smaller, hence, after infinitely many generations, transformed images of the initial figure reduce to points. This set of points constitutes the final fractal image.

The way by which patterns are created by this method, namely the initiation from a large scale and the propagation. towards successively smaller scales in an organized and systematic manner, allows us to postulate an intuitive analogy with a natural fracturing process. In general, fractures occur perpendicularly to the minimum stress in a rock mass. After fracturing, the released maximum stress repartitions in the rock mass. Fractures of each successive generation should be approximately perpendicular to the previous. Figure 47 shows the resulting network for six generations staj $\mathrm{ug}$ from an initial square. This pattern is very different from the classical Warren and Root [97] model. Here, except for the sample size, there is no characteristic length dimension, for the pattern of fractures. Instead, the system posseses a hierarchy of scales, a cascade of fractures and an inherent self-similarity. In particular, the number of fractures at each generation increases (here, it doubles), while their width decreases ( here, it becomes approximately half every two generations). We must point out that fracture widths in all the patterns presented in the figures have been exaggerated to enhance graphical display.

The imaga of Figure 47 is fractal. Indeed, this image can be reproduced by shrinking the whole pattern, by a certain factor, rotating it and putting it back on the upper half of the original one. The same can be done for the lower half, leaving a narrow empty space between the two. Each 


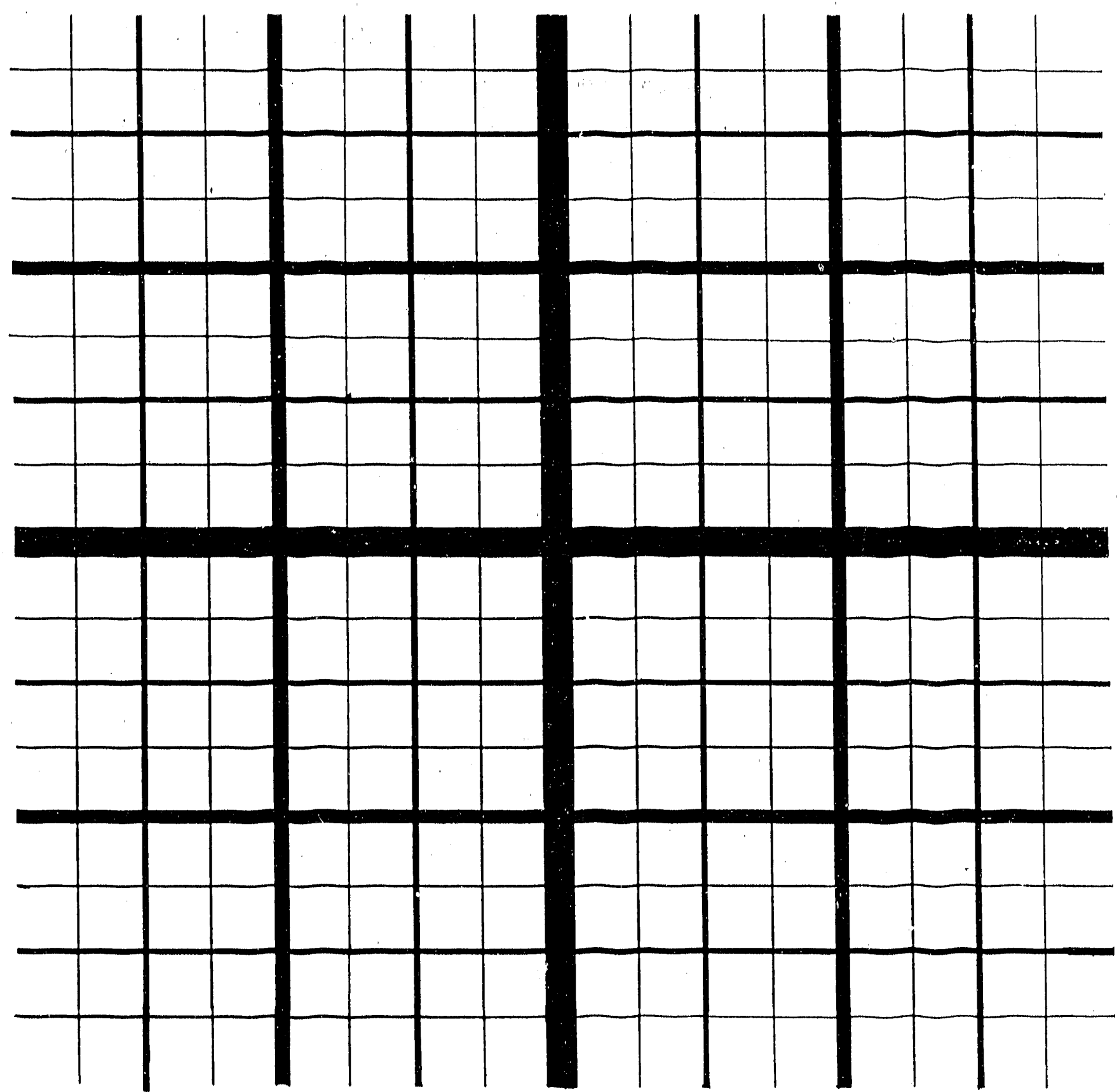

Figure 47: Fractal pe.ttern produced by perpendicular systematic fracturing of an initial square. Six generations of fractures are shown. The initial fracture is parallel to the top and bottom of the map. Fracture widths are exaggerated. 
and every piece of unfractured material is the image of the initially unfractured square under a given set of known numerical transformations. We must point out, that this particular network of fractures has a reproducible geometry because no random noise was inserted during iterations. Thus the geometric characteristic of each fracture, including their connectivity, is deterministic. Introduction of randomness is certainly possible and could result into more realistic-looking images. This is currently in progress.

While the pattern of Figure 47 is rather orderly, consisting of neatly arranged perpendicular fractures, fractal geometry also allows the introduction of variations in the angle of intersection of fractures of different generations, as well as for non-rectilinear and rugged-edge fractures. This is achieved by the use of non-conformal transformations.

The repetitive pattern in Figure 47 consists of one fracture, that divides in two parts an otherwise unfractured block. The angle of the initial fracture controls in part the geometry of the final pattern. If the angle between the initial fracture and the bottom and top edges is taken greater than zero, Figure 48 results. The relative complexity of this new pattern hides the fact that there is only a trivial difference with Figure 47, namely the angle of the initial fracture. This angle can be arbitrary, but not very large, otherwise the resulting pattern bears no resemblance to a set of natural fractures. This limits somewhat the options for simulation of natural patterns, and could be associated to a physical fracturing process. Besides its orientation, the shape of the initial fracture also dictates the properties of the final pattern. Figure 49 results from a pattern similar to Figure 48 , except that the fracture is tapered to render the whole system hydraulically unconnected. Other shapes of the initial fracture can be included to create widely different patterns.

Although a square was used to initiate the process in the previous patterns, this is not a requirement of the technique. Different shapes may also be used. Using an arbitrary shape as the initial pattern makes the hyperbolic terms in the transformation more important. This results into curved shapes and non-parallel edges for the blocks. Figure 50 is an example of the same basic pattern as in Figure 48 except that the initial block is trapezoidal instead of square. Despite the apparent complexity, every piece of the rock in the pattern is still the image of the initial unfractured trapezoidal block under the specified set of transformations.

At this point, it is appropriate to remember that we have explored only variations of the simplest case possible: a quadrilateral medium with a single initial fracture. The number of sides of the total 


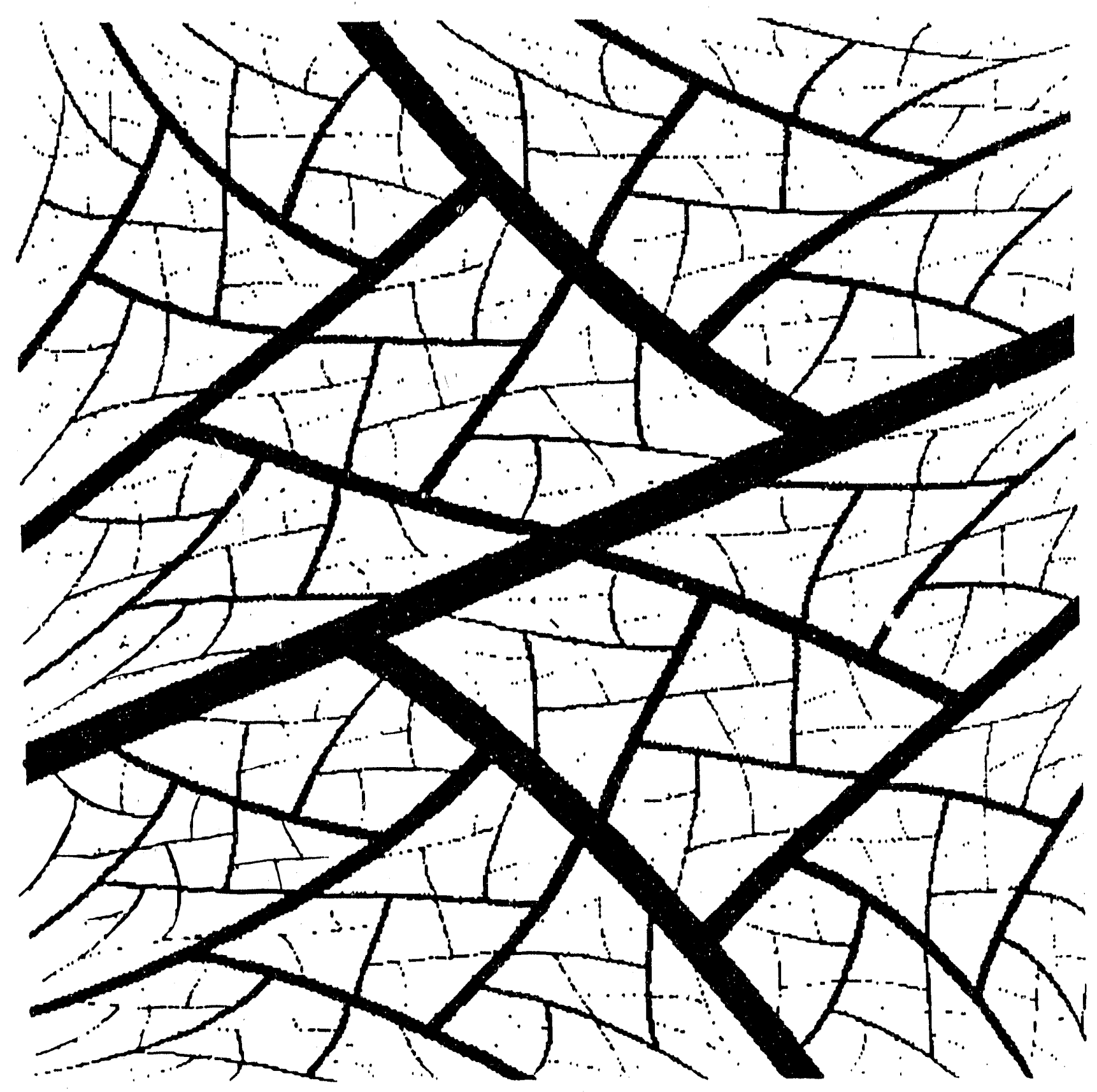

Figure 48: Fractal pattern produced by systematic fracturing of an initial square. Nine generations of fractures are shown. The initial fracture is at a given angle with respect to top and bottom of the map. Fracture widths are exaggerated. 


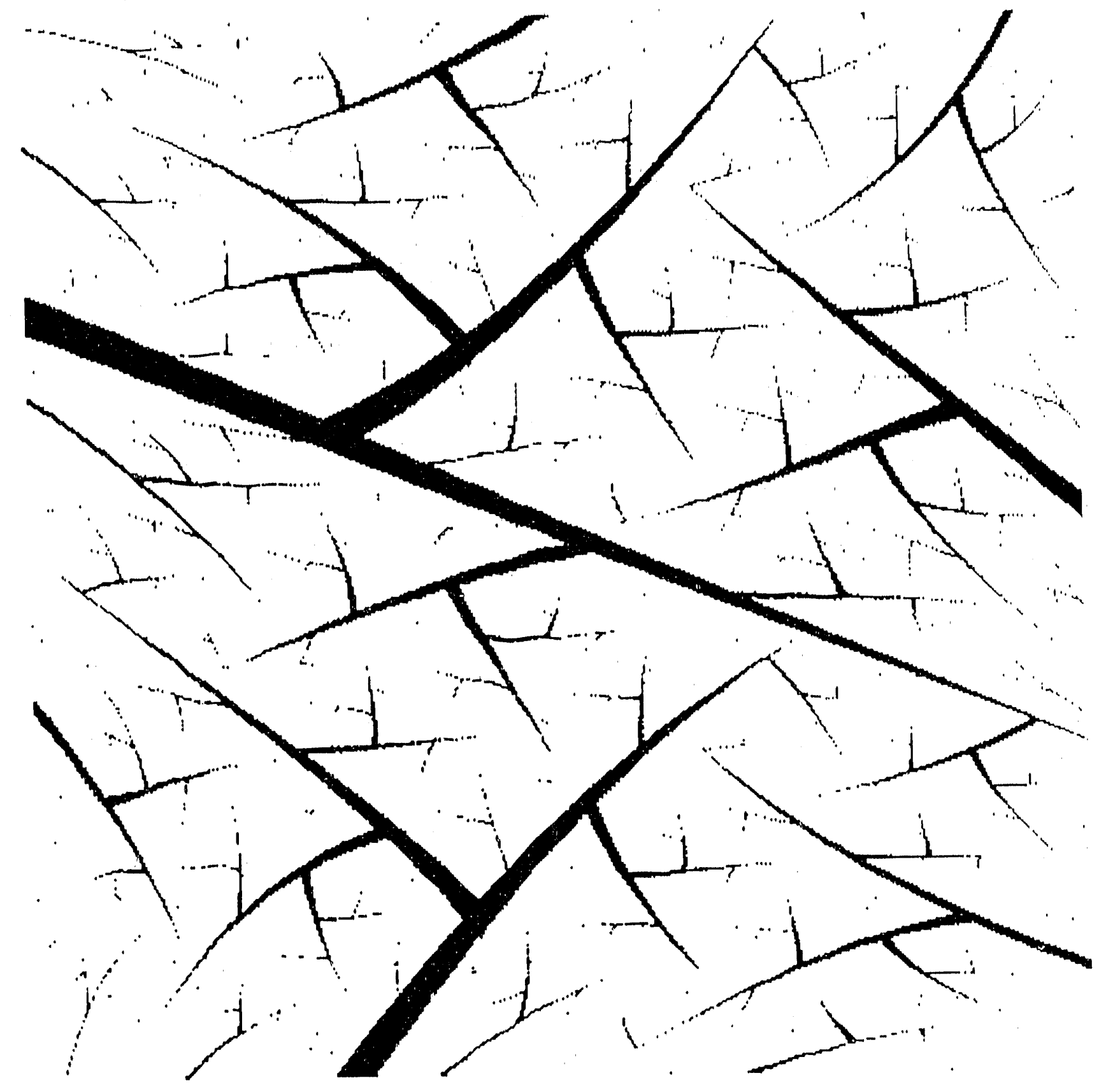

Figure 49: Fractal pattern produced by systematic fracturing of an initial square. Six generations are shown. The initial fracture has tapered shape and it is at an angle with respect to top and bottom of the map. Fracture widths are exaggerated. 


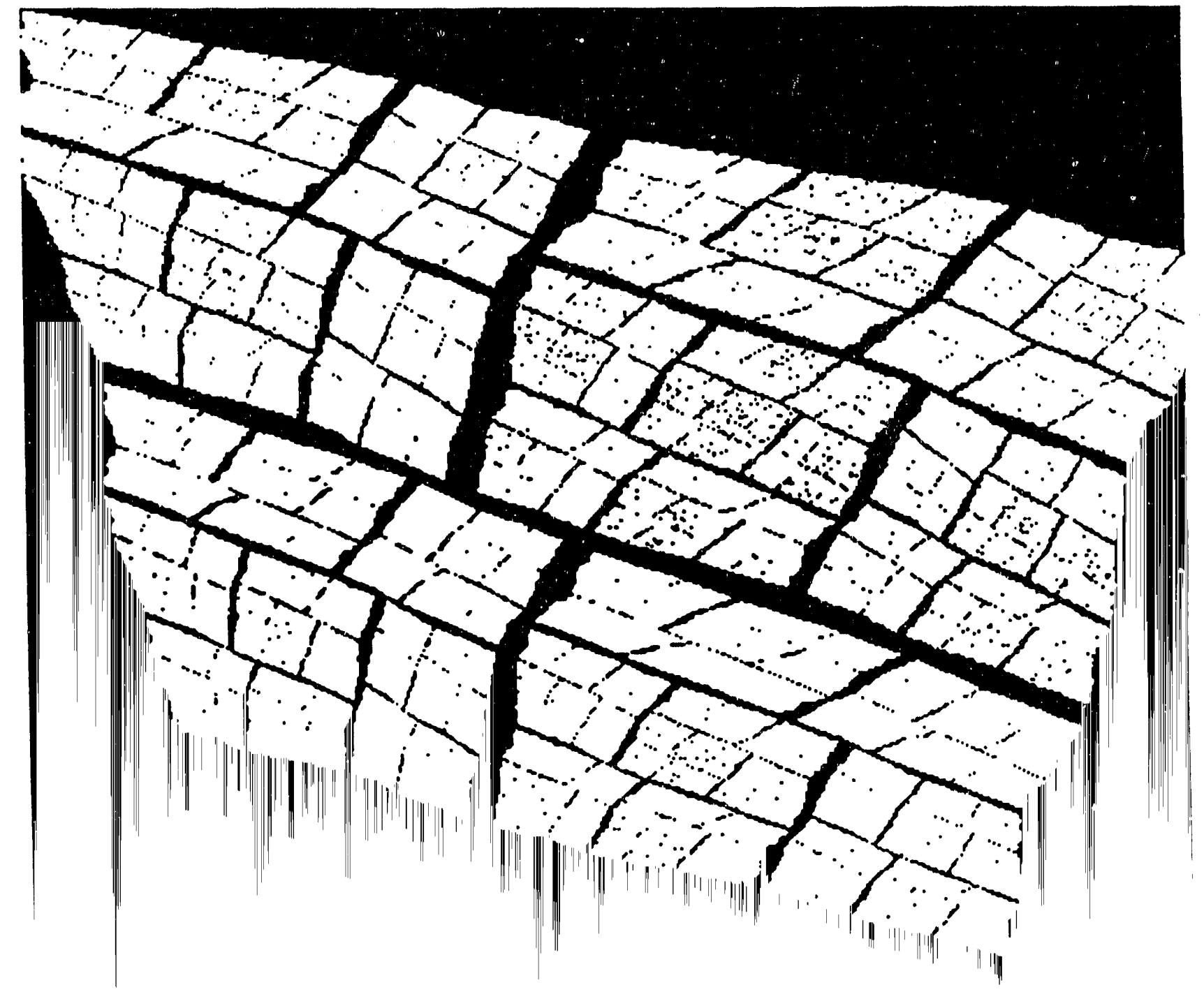


object is, in principle, left to the user to select. Here, we choose only four-sided patterns because the obtained patterns are interesting enough and because they are simple to generate. Instead of one initial fracture, one could selec a number of fractures, which could intersect each other. Also, the original shape could be polygonal of any number of sides. Of course, increase in complexity results into more elaborate computations. The number of transformations in the set equals the number of parts in which the initial unfractured medium is divided.

The initial pattern of Figures $47-50$ is a quadrilateral shape divided by a single fracture. This process results in two parts in the original map, therefore two transformations are needed. Each transformation consists of two equations, one for the $x$ coordinate and one for the $y$ coordinate of the two-dimensional space. The following bilinear transformations were used for the iteration

$$
\begin{aligned}
& x^{\prime}=a x+b y+c x y+d \\
& y^{\prime}=e x+f y+g x y+h
\end{aligned}
$$

where:

$x^{\prime}, y^{\prime}=$ cocrdinates of a given point in the $n$th generation after the application of the map,

$x, y=$ coordinates of the point in $(n-1)$ th generation to be mapped,

$a, b, c, d, e, f, g, h=$ coefficients of the transformation.

The values of the coefficients for every transformation contain all the information needed to reproduce the fractal image. The transformation shown above corresponds to a final pattern with four sides, polynomials with four coefficients being the appropriate mathematical expressions to map the initial four vertices into successive generation. Another feature that characterizes the patterns generated and it is very useful for fluid flow purposes, is the systematic connectivity of the fractures. Every fracture in Figures 47-50 abuts a fracture of a previous generation. This property originates from the common information shared for these four patterns, that is, the same shape in the initial basic design. Generalization to three-dimensional objects is relatively easy, although it is not discussed here. This can be accomplished by an additional coordinate in the transformations and a three-dimensional instead of a two-dimensional initial shape. 


\subsection{FLUID FLOW SIMULATION}

\subsubsection{Construction of Conductivity Matrix}

The usefulness of any synthetically created network for fluid flow purposes strongly depends on the structure of the correspondins flow conductivity matrix and on the ability to solve fluid flow problems. As in any other simulation, assumptions regarding the nature of flow in each fracture and in the network need to be made. For numerical solution of the flow problem, a finite number of generations must be considered. Analytical methods can be used for extrapolating the numerical results to a higher precision. As discussed above, to reproduce a pattern the following must be known:

a) The initial polygonal shape.

b) The set of transformations used for the particular fractal image.

c) The sequence in which the transformations where applied.

In the cases of Figures 47-50, only two transformations are used, denoted by 0 and 1, respectively. The sequence of transformations for a block of the $n$th generation will consist of a combination of $n$ 0 's and 1's in a given sequesace. This way of assigning addresses to the parts of the fractal pattern is discussed in Barnsley [6]. A pattern containing $n$ generations of fractures will be composed of $2^{n}$ pieces. The unique binary sequence that identifies each piece, makes possible to precisely specify the address of a given fracture in the pattern, its shape and location, as well as to devise a numbering system for the end points of that particular fracture. In this fashion, we identify the nodes of the network. We point out that this is an important development, as it alleviates the need for a finite-difference or finite-element description and coarse-grid averages. Moreover, it is uniquely related to the self-similar, nested structure of the object.

The fact that curved and rouged-ed e fractures are possible with this technique calls for assumptions more general than the standard cubic law, in order to describe flow through the fracture and the network in general. This is an issue not exclusive to this problem and requires separate investigation. Here, we will study the pattern of Figure 48 which has approximately rectilinear fractures. Using this as an example, our objective is to show how to apply a systematic approach to the solution of the fluid flow problem.

We consider steady-state, single-phase flow in the network of fractures. Along each fracture we assume that the following linear expression applies: 


$$
Q=C W^{\lambda}\left[\frac{\Delta P}{L}\right]
$$

where:

$$
\begin{aligned}
& Q=\text { Volumetric flow rate } \\
& W=\text { Width of fracture } \\
& L=\text { length of fracture } \\
& \Delta P=\text { Pressure drop along the fracture } \\
& C=\text { Constant } \\
& \lambda=\text { Conductivity exponent. }
\end{aligned}
$$

The conductivity exponent is usually taken equal to three, although values as high as eight have been reported [57].

To construct the conductivity matrix, we first assign addresses to the two end nodes of the initial fracture. According to the previous, they are node 1 and node $2^{n+1}-2$ respectively. Each new fracture that appears from now on, subdivides two blocks. The general expression for the node addresses in Figure 48 is

$$
\begin{gathered}
\alpha=1+\sum_{i=1}^{j-1}\left(2^{n+1-i}-1\right)^{a d_{i}} \\
\beta=2^{n+1}-2-\sum_{i=1}^{j-1}\left(2^{n+1-i}-1\right)^{1-a d_{i}}
\end{gathered}
$$

where:

$\alpha=$ Number of the first end node of the fracture

$\beta=$ Number of the second end node of the fracture

$j=$ Generation to which blocks separated by this fracture belong

$n=$ Total number of generations of the pattern

$a d_{i}=i$ th number (zero or one) in the sequence that defines the address of blocks at each side of the fracture.

With the exception of the last digit that differentiates the two blocks, the address of a fracture is identical to that of the blocks. Suppose, for example, that we are dealing with a fracture pattern like Figure 48 but only with three generations in total. The initial fracture divides the medium in two 
blocks whose identifying codes are 0 and 1 . These are the addresses of the first generation. Using formulas (67) and (68) the node numbers are 1 and 14. The second generation creates four blocks with codes $00,01,10$ and 11 . The fracture identification codes are 0 and 1 , respectively. Equations (67) and (68) applied to fracture 0 give 2 and 7 respectively. The same equations applied to fracture 1 give 8 and 13 respectively. The same is repeated for the eight blocks of the third generation that form four more fractures. These fractures will be denoted by 00, 01, 10 and 11, while their end node numbers will be 3 and 4,5 and 6, 9 and 10, and 11 and 12, respectively. The coordinates of each node are obtained by the address of the fractures. Thus, we obtain the coordinates and number of each node in the network. As more fractures are added, and internal nodes appear in already existing fractures, simple relationships of these nodes with the end nodes can also be found in a systematic way. The final step is to calculate the conductivity of each fracture according to the assumptions made and to fill the entries of the conductivity matrix.

The conductivity matrix thus constructed exhibits a certain self-similarity. Figure 51 is a schematic of the structure of the conductivity matrix for the pattern of Figure 48 for one, two and three generations of fractures. It is evident that the matrix shape for generation consists of the matrix of the previous $(n)$ generations superposed twice along the main diagonal, along with the addition of a few new terms at both ends of the main diagonal and a few terms at offdiagonal positions. This is a mathematical expression of the fact that the pattern is formed by the superposition of two smaller patterns of the same internal connectivity and with new elements that bond the patterns together.

Once the conductivity matrix has been obtained we may solve the steady-state problem. The solution at transient conditions requires an additional calculation of the volume associated to each fracture. Although this is readily done, we concentrate in this report on the steady-state solution only.

\subsubsection{Steady State Flow}

A true fractal pattern of fractures contains an infinite number of generations. On the other hand, the numerical solution requires that a finite number of generations only be considered. Recalling that the size of the conductivity matrix doubles with every new generation, it becomes evident that the conductivity matrix must be kept at a reasonable size. The effect of the number of generations 

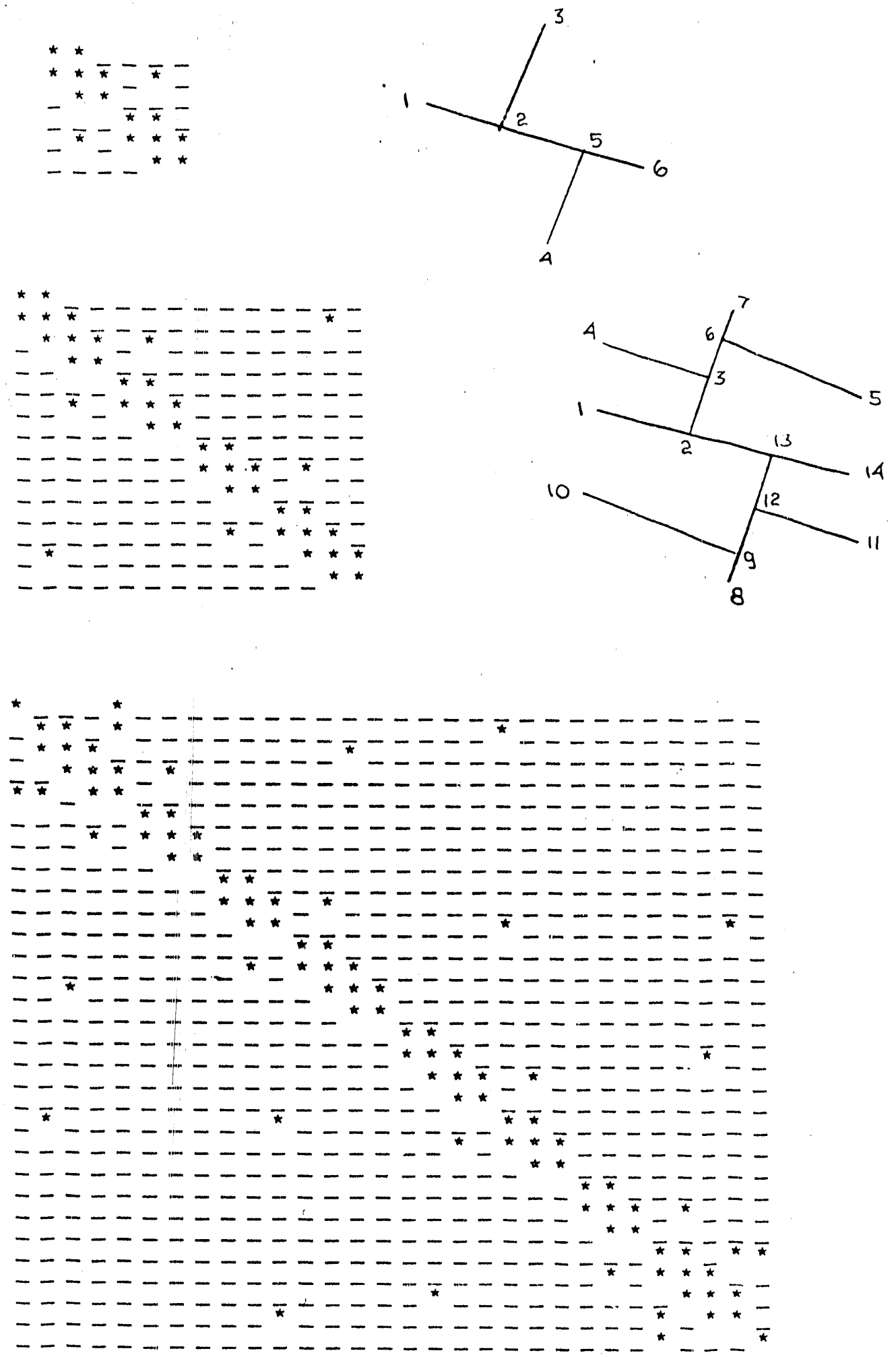

Figure 51: The conductivity matrix for one, two and three generations of fractures. 
on the total conductivity of a fractal fracture medium needs to be considered. For some preliminary insight, it is instructive to solve the closely related problem of the pattern of Figure 47, which admits an analytical solution .

We impose no-flow boundary conditions at the top and bottom of Figure 47 and apply a pressure gradient from left to right. Due to the symmetric configuration of the system, only fractures along the pressure gradient direction carry flow. In general, it is easy to demonstrate that the total volumetric rate for $2 S^{\lambda}<1$ is

$$
Q=Q_{0} \sum_{i=0}^{\infty}\left(2 S^{\lambda}\right)^{i}=\frac{Q_{0}}{1-2 S^{\lambda}}
$$

where:

$Q=$ Total volumetric flow rate

$Q_{0}=$ Flow rate corresponding to the initial fracture only

$S=$ Ratio of sizes between a given region and its map in the following generation. This value is close to but less than 0.5 for this specific example.

The above equation predicts that the volumetric flow rate converges to an asymptotic value as the number of fractures increases. The rate of convergence is dictated by thte conductivity exponen. For a general pattern, a numerical solution is necessary. We have made such calculations for the pattern of Figure 48. A behavior close to the theoretical result is expected. Numerical results are shown in Figure 52 for various values of the conductivity exponent. The rate of convergence is slower than that of the simpler pattern of Figure 47 , but the asymptotic value is similar. For values of the conductivity exponent grater than or equal to three, convergence is reached after a few generations. The practical importance of this is that, at least for steady state, it is not necessary to consider infinitely many generations. Thus, conductivity matrices can be kept to a reasonable size. In our scheme, the higher the value of the conductivity exponent, the smaller the contribution of smaller fractures to the total conductivity.

An important feature of the fractal objects is the dependence of total conductivity on the size of the system. According to equation (69) this relationship is the same with what exists between the flow rate for the initial pattern $Q_{0}$ and the size of the system. Indeed, if we increase each of the two geometrical dimensions of the system by a factor $\epsilon$, at constant pressure gradient, the flow rate $Q_{0}$ will increase by a factor $\epsilon^{\lambda}$ 


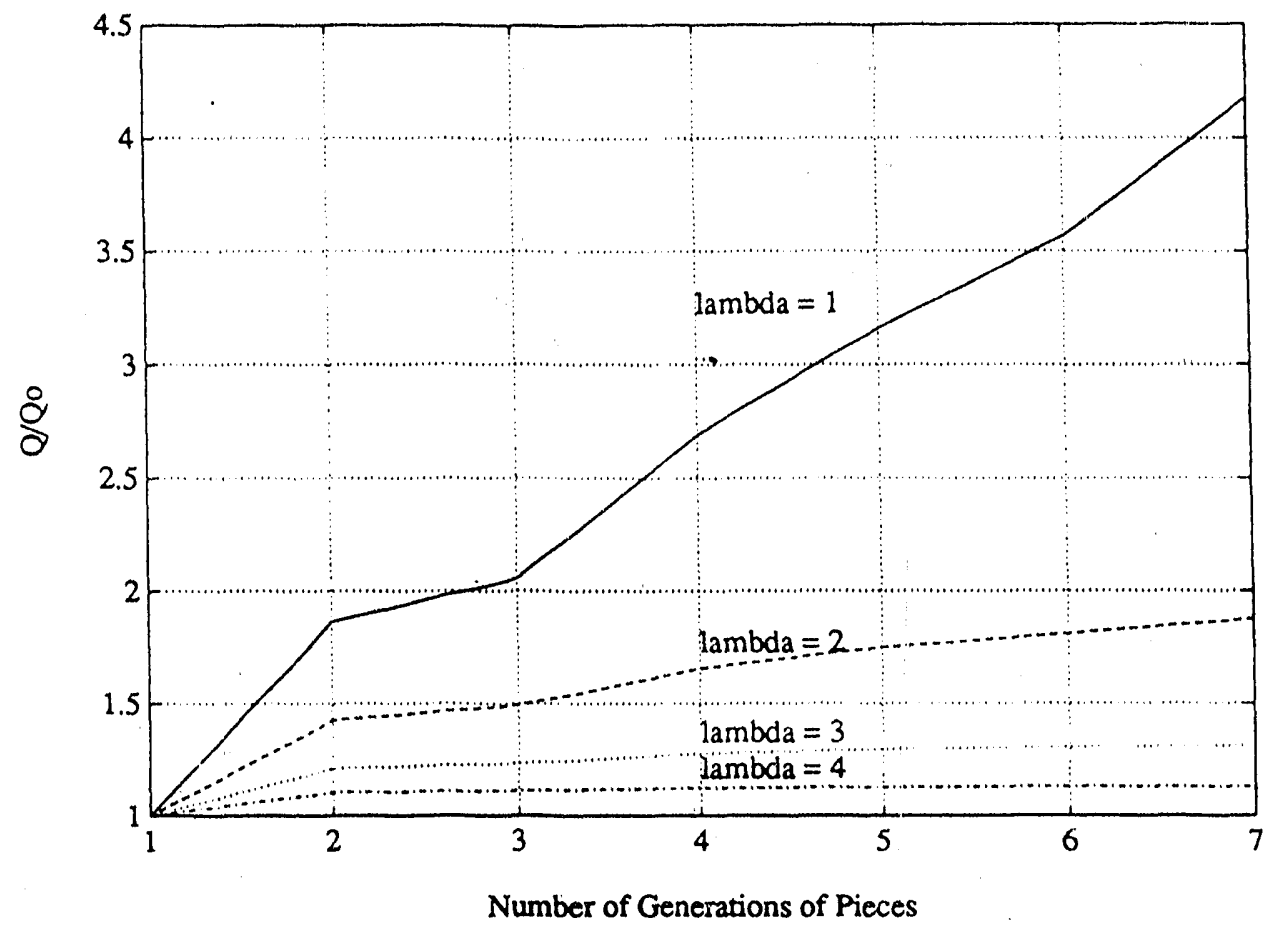

Figure 52: Asymptotic behavior of the total conductivity with increasing number of generations of fractures.

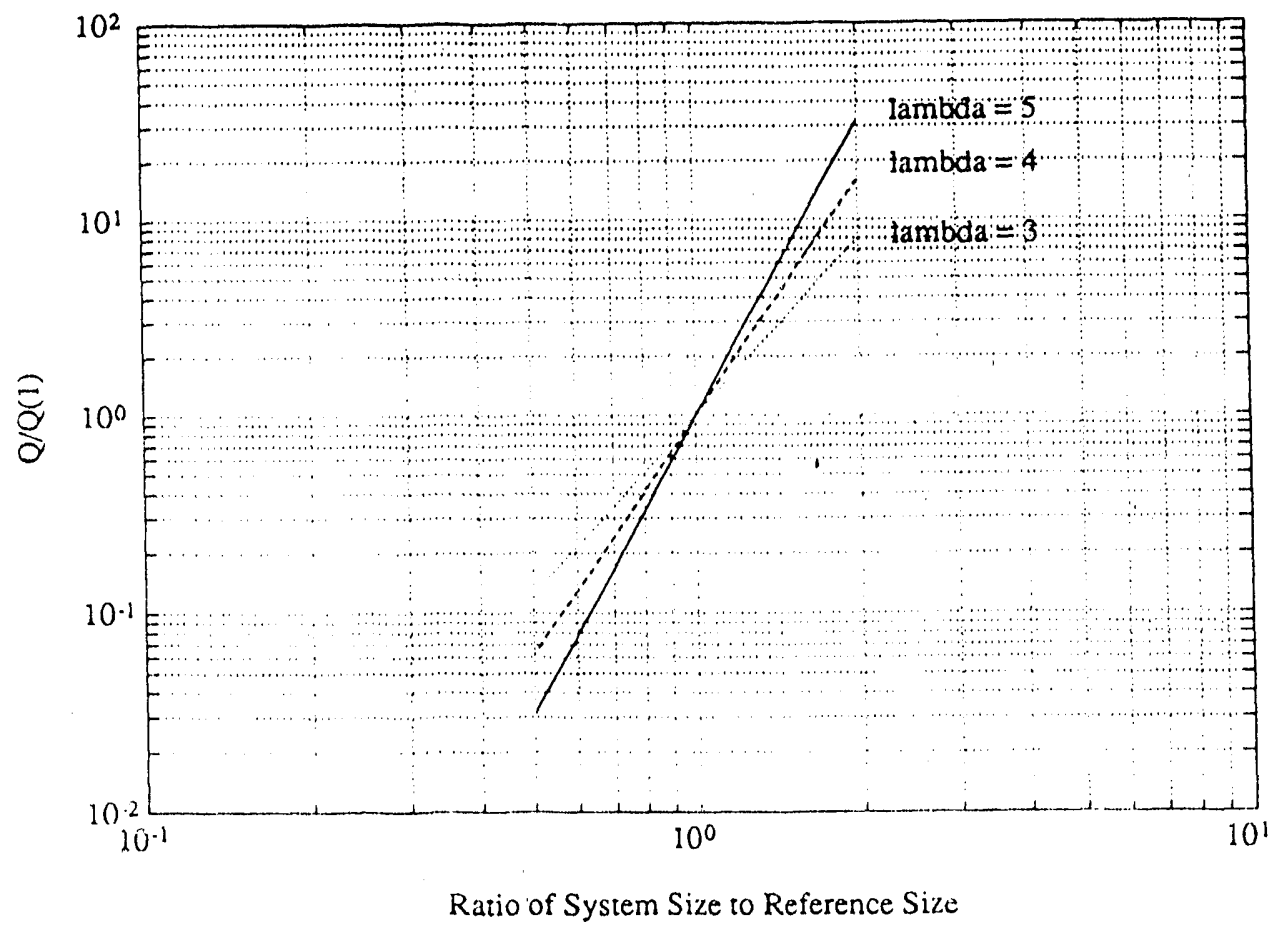

Figure 53: Total conductivity versus system size. 


$$
Q_{\epsilon}=\epsilon^{\lambda} Q_{1}
$$

where:

$$
\begin{aligned}
& Q_{\epsilon}=\text { Flow rate at scale } \epsilon \\
& Q_{1}=\text { Flow rate at reference scale. }
\end{aligned}
$$

The validity of this was investigated for the pattern of Figare 48. Steady-state fluid flow was simulated in three systems of different size. The power-law relationship of the total conductivity with scale was verified, the exponent of the power-law relationship being equal to the conductivity exponent, $\lambda$, as expected (Figure 53). This dependence of the total conductivity of the system on size is a common feature of fractal systems and it has been used in a different context for the description of pressure transients in such systems [14].

\subsection{CONCLUSIONS}

Fractal patterns of fractures in two dimensions can be created by techniques for the creation of fractal images [6]. The application of this methodology to the generation of models of fracture networks is still being developed. The next step in our study is the creation of fractal fracture networks of a specified fractal dimension. All patterns presented in this report have a fractal dimension in the range 1.95 to 2 .

This method of generating fractal networks appear to be powerful for several reasons:

a) The generated patterns appear more realistic than conventional regular or random networks.

b) The systematic construction of the patterns allows for the consideration of specific rules of fracturing.

c) The self-repetitiveness of the pattern is also reflected in the conductivity matrix, provided that a convenient scheme for the enumeration of the nodes is selected. This facilitates the construction of the conductivity matrix.

The study of the steady-state solution for a typical fractal network reveals that the total conductivity of the system approaches an asymptotic value. The conductivity exponent $\lambda$ aictates the minimum number of generations necessary to achieve convergence, as well as the final asymptotic value of the total conductivity. Convergence is rapidly achieved for higher values of this exponent. Finally, the total conductivity of the system is scale-dependent. Likewise, this dependence is 
strongly influenced by the conductivity exponent. 


\title{
6 LARGE SCALE AVERAGING OF DRAINAGE AT LOCAL CAPILLARY CONTROL
}

\author{
Y. C. Yortsos, C. Satik, J.-C. Bacri and D. Salin
}

\subsection{INTRODUCTION}

The success of effective continua for transport in macroscopically homogeneous media rests on the condition that the scales at the microlevel and the level of the continuum are separable. The process is then homogenizable. This condition is readily detected in previous averaging schemes, whether based on closure postulates (Bear et al. [8] Whitaker [99]) or on asymptotic expansions (Auriault et al. [4]). For practical implementation it is also required that coefficients, such as capillary pressure and relative permeabilities in immiscible displacement, can be defined and computed. Unfortunately, however, the simultaneous satisfaction of these conditions is intrinsically impossible in present continuum models.

Scale separation requires that the microstructure is statistically homogeneous over the averaging volume, therefore that the correlation length of the microscale heterogeneities, $\lambda$, is sufficiently smaller than the scale of averaging, $\ell(\lambda \ll \ell)$. The ratio $\lambda / \ell$ may be recognized as the small parameter in perturbation expansions (Bensoussan et al. [10]). In the absence of flow, this becomes a constraint on the geornetrical (pore) structure, which is usually satisfied in random porous media.

This is not sufficient, however. Long-range correlations are also set by th; process itself, even in a medium with uncorrelated structure. This is the case for processes such that scale-dependent viscous or gravity forces compete with capillarity at the microscale (e.g. for sufficiently large capillary or Bond numbers, $N_{c a}$ or $N_{B}$ ) . The problem is then not amenable to averaging or homogenization (Auriault et al. [4]). To circumvent this difficulty, present continuum models rely on the premise of local capillary control. Indeed, such condition is a basic postulate cf conventional averaging schemes. The requirement can be formulated by requesting that $\lambda_{c} / \ell \ll 1$, where $\lambda_{c}$ is the length scale where capillarity and viscous forces are comparable

$$
\lambda_{c} \sim \frac{\sqrt{k}}{N_{c a}} \text { or } \frac{\sqrt{k}}{N_{B}}
$$

The above applies for processes in random media away from percolation. Enforcement of local cápillary control can certainly be accomplished at sufficiently small $N_{c a}$ (or $N_{B}$ ). 
Even ihen, however, difficulties arise. Drainage dominated by capillarity has been known to be a percolation process (Gennes and Guyon [22], Lenormand and Bories [51], Wilkinson and Willemsen [102]). Near the onset of the displacement, where the displacing phase reaches macroscopic connectivity, a percolation threshold is approached. The displacing fluid acquires a fractal structure (that of the percolation cluster, Feder [28]), correlation lengths diverge and averages become dependent on the averaging scale. Therefore, near percolation, even in the absence of viscous effects, the conventional continuum formalism breaks down (see also Lenormand et al. [52], Lenormand [50] and Sahimi and Yortsos [76]).

It follows that for effective continua to be applicable, the two-fold requirement of local capillary control away from percolation thresholds must be strictly enforced. Now, in all continuum schemes, the evaluation of transport coefficients is, in principle, possible by solving appropriate boundary value problems at the microlevel. In fact, the adoption of pore networks to represent the pore structure has reduced some of these to equivalent electrical resistor problems (Koplik [47]). While this approach may work well in certain cases, it fails in displacement processes. Here, the computation of effective properties, such as capillary pressure or relative permeabilities, requires that the distribution of fluid phases in the pore space is known a priori. The latter is dependent on the displacement history, during the course of which a percolation process was, in all likelihood, encountered (e.g. in primary drainage). It follows that present continuum methodologies are intrinsically deficient. Instead, statistical model based on percolation theory have emerged as the proper alternatives (Dias and Wilkinson [23], Heiba et al. [33], Heiba et al. [34], Parlar and Yortsos [61], Parlar and Yortsos [62], Wilkinson [101]) to supply the required coefficients.

The above have been applied to macroscopically homogeneous media, the disorder being in the pore size distribution, over which homogenization (averaging) is taken. However, identical issue, also arise in the large scale averaging of macroscopically heterogentous media. In this case, the disorder is in macroscopic parameters, such as absolute permeability, relative permeability and capillary pressure functions, provided that the latter two can be defined (condition of local capillary control). Averaging at this larger scale leads to effective ("poudo") functions, to be used for a coarse description over volume elements sufficiently large for scale separation to apply. As previously discussed, the foremost constraint for this is on the correlation properties of the microstructure, which in this case is the permeability field. Although field evidence irscreasingly 
points out to the existence of long-range correlations in geologic media (Hewett [36], Koch and Brady [46], Sahimi and Yortsos [76]), a condition that automatically negates separation of scales, we shall assume in this paper uncorrelated (or small correlation length) media. The case of fractal statistics, while of strong interest, requires a separate study.

In the general case, capillary control at the microlevel may not be postulated, thus a non-iocal description is necessary. This problem is centrai to dis placements in porous media at any level, whether miscible or immiscible (Hewett and Behrens [37]), and it is currently unresolved (although certain progress has been made in the case of passive dispersion, see Koch and Brady [45], Koch and Brady [46] and Yortsos and Chang [105]). Methods presently available for large scale averaging based on volume averaging (Quintard and Whitaker [69]), rely on the premise of local capillary control. However, it was pointed out above, that even under this condition, these formalisms a e not sufficiently adequate, due to their intrinsic inability to account for displacement history and the apprcach to percolation. Recent works by Amaziane and Bourgeat [2] and Quintard and Whitaker [70] provide some useful analysis for the non-local problem, although additional research is certainly needed.

In this paper we propose an altermative approach to large scele averaging under local capillary control, based on an extension of the percolation methods used for pore-level modeling. As discussed, capillary control is common to all previous schemes, although it is increasingly more difficult to be obeyed as the averaging length scale increases. Constraint (71) serves as a guide to estimate the conditions necessary for its validity. We shall consider application to primary drainage only, an immiscible displacement process that is well understood (Lenormand [50]). As in the case in pore-level models, the analysis will be restricted to random, isotropic and spatially uncorrelated media. The formalism is discrete, based on a lattice representation of the system, to which percolation concepts are applied. In a sense, the present is a scale-up of pore-level percolation processes. Non-trivial, novel results are obtained regarding the macroscopic saturation distribution, the onset of macroscopic displacement, its history dependence and the existence of large scale trapped saturations, none of which are possible with the previous methods.

The organization of the paper is as follows: After formulating the approach, the large scale saturation distribution at local capillary control is derived. Effective ("pseudo") capillary pressure and relative permeabilities are next obtaired. Numerical examples illustrate various features of the 
large scale averages.

\subsection{FORMULATION}

We consider a macrosr pically heterogeneous, randomly isotropic and spatially uncorrelated medium. Porosity, permeability and, in general, the geometric microstructure are the variables which are distributec, a spatially uniform and cirong wettability assumed throughout. Extension to variable wettability is in principle straightforward, although not attempted here. For convenience, but without loss in generality, we also assume that properties, such as capillary pressure, are distributed following the disorder of only one spatially varying parameter, the single-phase permeability. It is straightforward to extend the method when more variables are involved, but this would only serve to complicate the presentation.

We take $f(k)$ to be the probability density function ( $p d f)$ of the permeability $k$ on a volume basis, with $f(k) d k$ being the volume fraction with values in the interval $(k, k+d k)$ (Figure 54). Primary drainage of this macroscopically heterogeneous medium is considered. The representation is next taken that the medium consists of an ensemble of regions, each of which has its own characteristic structural properties (such as pore size distribution, etc.). Assuming that the latter are random, uncorrelated and isotropic, primary drainage of a given region, in isolation and independent of other regions, e.g. as in a core sample, is described by percolation theory, for example as summarized by Lenormand [50]. Given its finite size $\ell$, said region is invaded by the non-wetting phase when its percolation threshold is reached. Finite-size effects on this threshold can be estimated from ordinary percolation theory (Wilkinson [100]), but they are negligible as the ratio $\sqrt{k} / \ell$ decreases (however, one should expect possible complications for wide pdfs $f(k)$ ). Thus, the capillary threshold and, upon further penetration, the capillary pressure curve can be obtained from a combination of the pore-size distribution and percolation theory, for example as elaborated by Heiba et al. [34].

To each region with value $k$ we associate a drainage capillary pressure curve $P_{c}\left(S_{w} ; k\right)$ expressed for simplicity by the Leverett relationship

$$
P_{i}\left(S_{w} ; k\right)=\frac{\gamma}{\sqrt{k}} J\left(S_{w}\right)
$$

Here, $\gamma$ denotes the IFT between the two fluids, $S_{w}$ is the local wetting fluid saturation, while $J$ is a (Leverett) function taken independent of $k$. These simplifications, particularly neglecting 


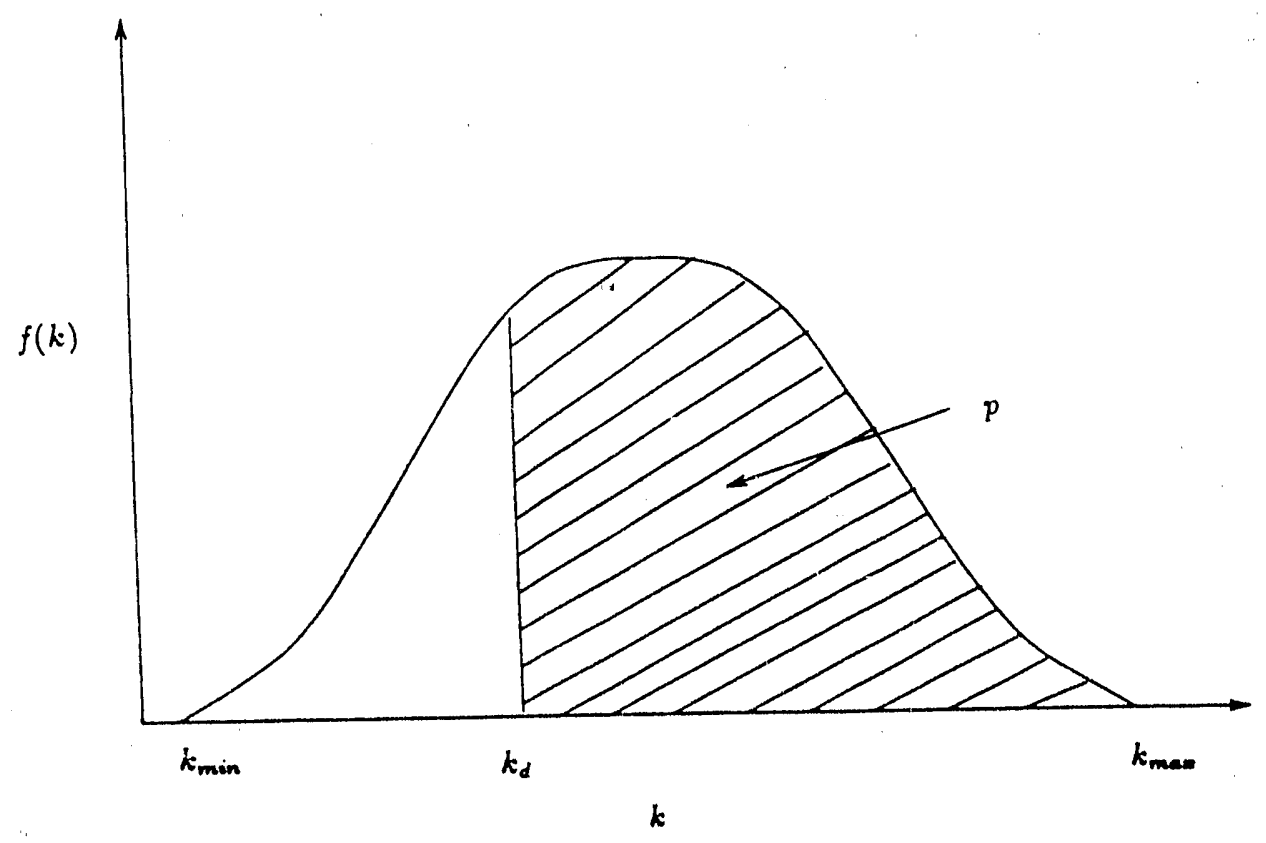

(a)

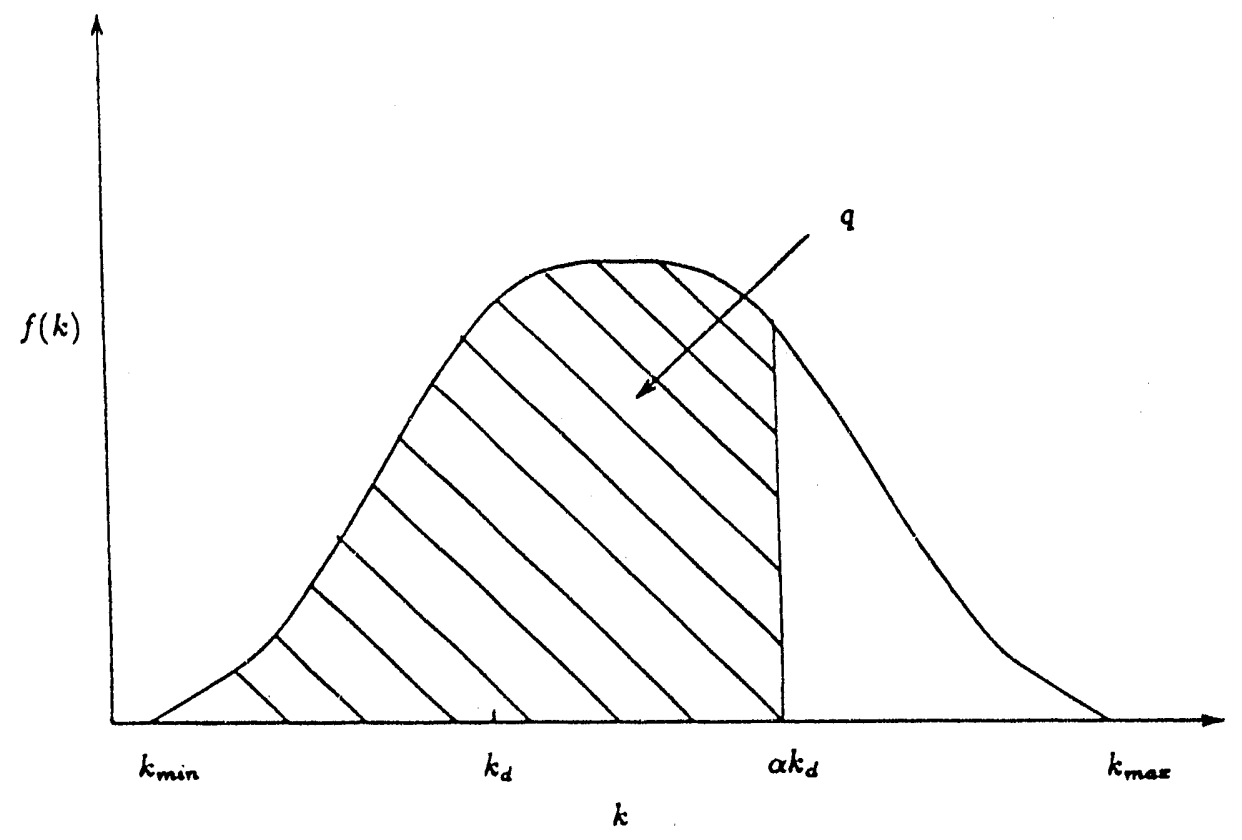

(b)

Figure 54: Schematic of permeability distribution: (a) Definition of fractioin $p$; (b) Definition of fraction $q$. 
any porosity variation, are taken for the sake of convenience, and they should not detract from the applicability of the proposed theory (see also discussion in Yortsos and Chang [105]). Typical schematics are shown in Figure 55a. Of particular interest is the capillary pressure threshold of any given region,

$$
P_{c d}(k) \equiv P_{c}(1 ; k)=\frac{\gamma}{\sqrt{k}} J(1)
$$

which is also spatially distributed. Its $p d f, F\left(P_{c d}\right)$, is simply

$$
F\left(P_{c d}\right)\left|d P_{c d}\right|=f(k)|d k|
$$

The relation between permeability and capillary pressure threshold was shown by Katz and Thompson [40] with the use of percolation concepts to be of excellent accuracy. Thus, the above assignment (72) - (73) is expected to be quite adequate for purposes of primary drainage.

The analogy between pore-level and large-scale averaging, is, now, evident. Each macroscopic region is analogous to a pore, the capillary threshold $P_{c d}$ being equivalent to a pore drainage radius (Dullien [26]). When the macroscopic medium is subject to primary drainage, any region can be invaded by the non-wetting phase, provided that the following two conditions are met: That at the current capillary pressure level, $P_{c}$, the threshold $P_{c d}(k)$ is exceeded; and that the region is accessible to the invading phase. The percolation aspects of this process are apparent. Furthermore, macroscopic regions drain completely when they reach residual saturation values $\left(S_{w r}\right)$, in which case they may block further drainage of surrounded elements. The latter is similar to the process of percolation with trapping discussed by Dies and Wilkinson [23] (also contained in Yortsos and Sharma [107]). At the same time, pore-level and large-scale problems are also different. In porelevel models, a pore body is assumed to completely fill upon invasion, its saturation content being a binary variable (zero or one). In contrast, the saturation of an invaded macroscopic region can take any continuous value between zero and one, as dictacted by its corresponding capillary pressure curve. This difference is controlled by the Leverett $J$ function, essentially through the ratio

$$
\alpha=\left[\frac{J\left(S_{w r}\right)}{J(1)}\right]^{2}
$$

since $J$ is monotonic (Figure $55 \mathrm{~b}$ ). 


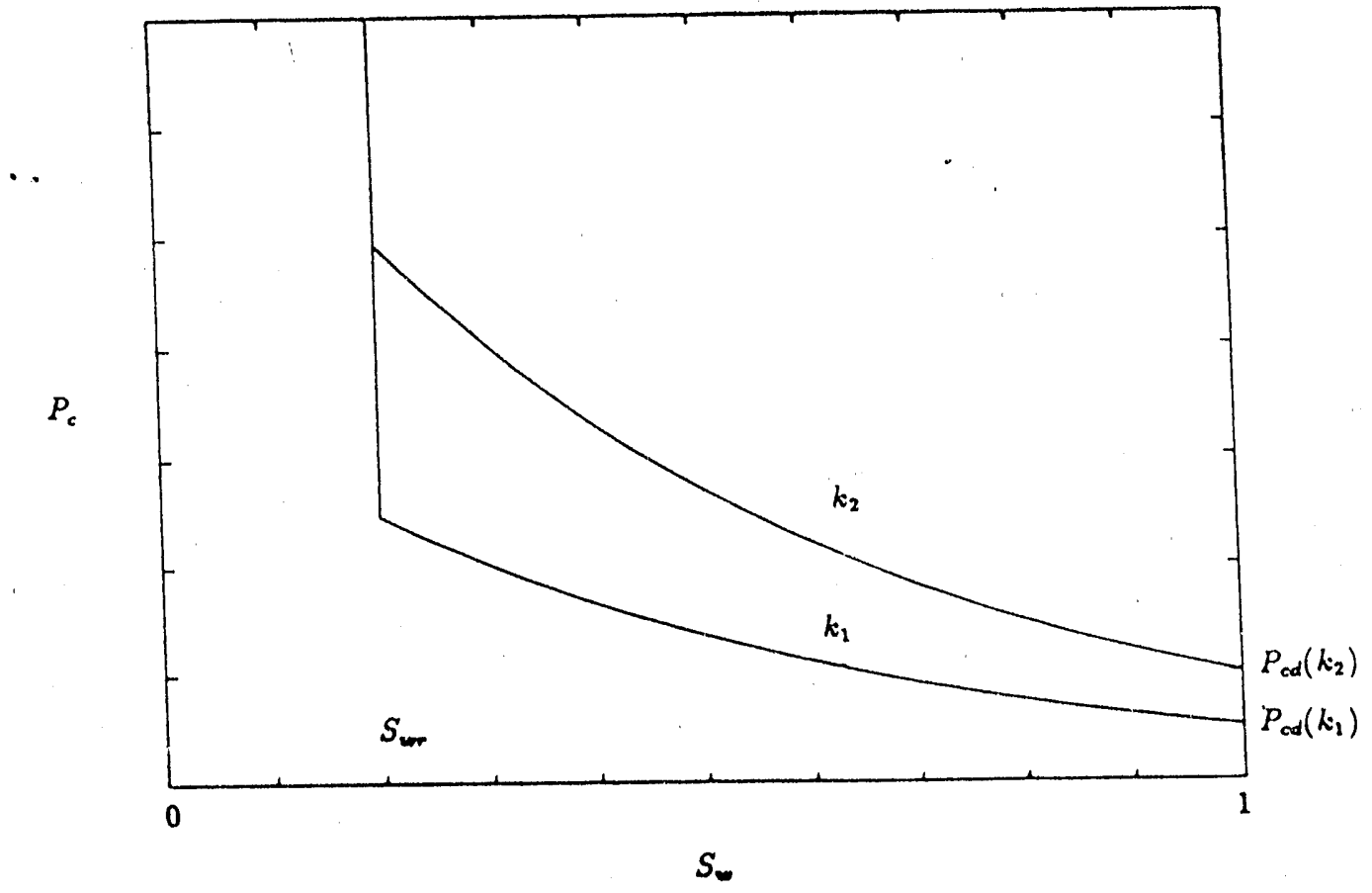

(a)

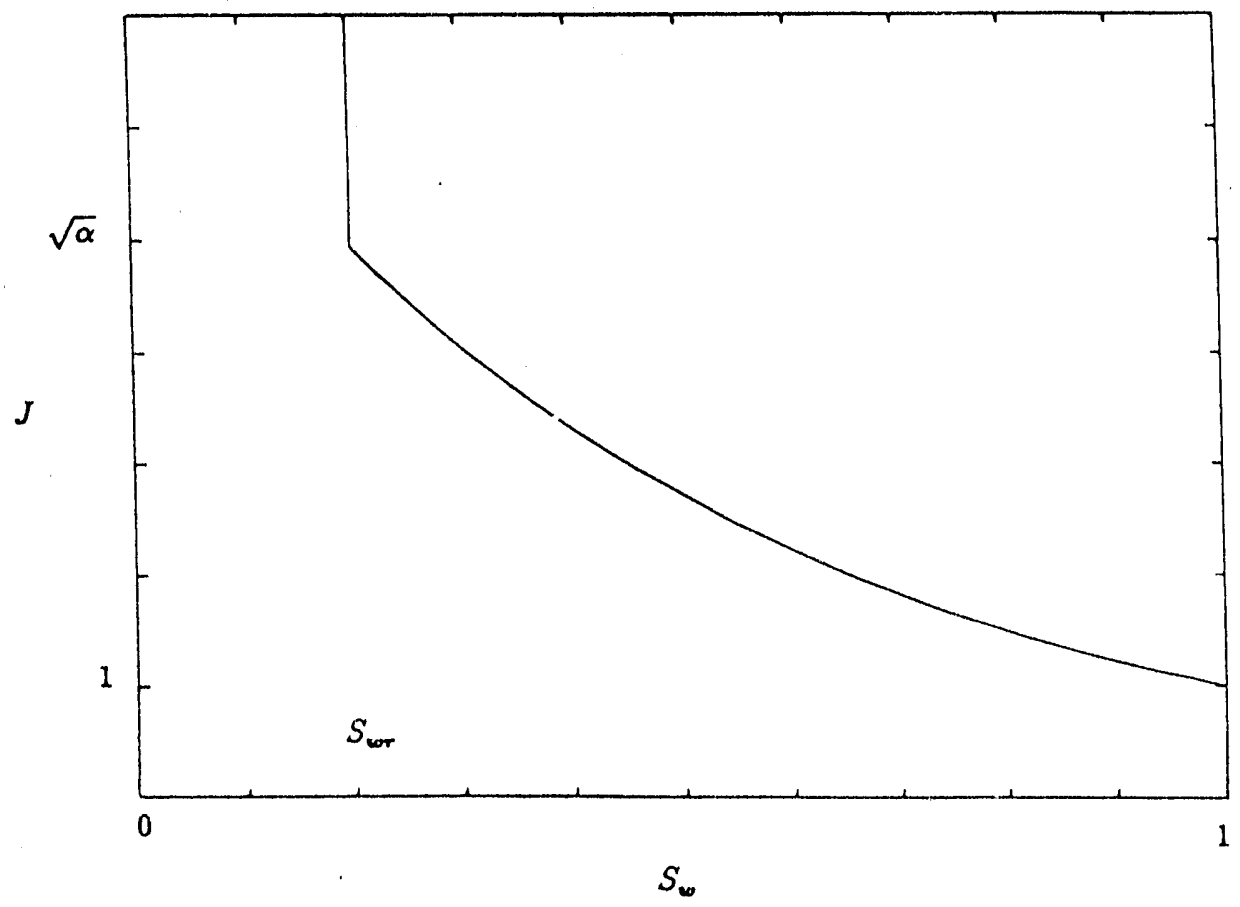

(b)

Figure 55: Schematics of : (a) Individual capillary pressure curves for two permeabilities (in arbitrary units); (b) the $J$ function. 
Evidently, the binary occupancy at the pore-level can also be obtained from (75) in the limit $\alpha=1$. The two problems are, therefore, different insofar as $\alpha>1$. This simple constraint, which originates from the application of percolation theory at the pore-level, differentiates the problems at the two levels and leads to novel results for large scale avarages.

From this discussion it is evident that large-scale averaging at local capillary control can be successfully approached using percolation concepts. To proceed, we shall denote large-scale quantities by brackets. Just as in a homogeneous sample, for the onset of drainage a large scale capillary threshold $\left\langle P_{c}\right\rangle_{d}$ must be exceeded. Subsequent drainage follows a large scale percolation behavior. Given a capillary pressure level $P_{c}$, draining or the wetting phase occurs in regions of perr eability $k \geq k_{d}$ that are both accessible to the non-wetting phase (belong to the percolation cluster) and not trapped (not surrounded by completely drained regions). Here, $k_{d}$ is a running variable related to the current capillary pressure value $P_{c}$ by

$$
P_{c}=\frac{\gamma J(1)}{\sqrt{k_{d}}}
$$

(see Figure 55b). Each of these regions drains according to its capillary pressure or $J\left(S_{w}\right)$ curve, hence its saturation content is variable. As previously noted, this is different from the analogous pore-level problem, where a given pore can be occupied by one phase only. Without loss in generality, we normalize by taking $J(1)=1$, which can be accomplished by a simple rescaling. An actively draining region has a wetting saturation given from the solution of

$$
J\left(S_{w}\right)=\frac{\sqrt{k}}{\sqrt{k_{d}}}
$$

where, $J$ itself displays percolation behavior near $S_{w}=1$. We shall denote the inverse of $J$ by $I$

$$
I\left(\frac{k}{k_{d}}\right) \equiv J^{-1}=S_{w}
$$

In general, an irreducible wetting saturation $S_{w r}$ typically exists (excluding thin film flow), such that $S_{w}=S_{w r}$ for $J \geq J\left(S_{w r}\right) \equiv \sqrt{\alpha}>1$ (Figure 55b). This implies that the function $I$ is also constant in the appropriate interval. To specify the latter we define, for given $k_{d}$, the permeability value $k_{r}\left(k_{\min } \leq k_{r}\right)$, above which a continuously draining region has reached $S_{w r}$

$$
k_{r}=k_{d} \alpha
$$


We summarize

$$
I(x)= \begin{cases}1 & ; x \leq 1 \\ S_{w} & ; 1 \leq x \leq \alpha \\ S_{w r} & ; \alpha \leq x\end{cases}
$$

For simplicity, all regions are taken in the analysis below with identical residual saturation values.

The features of the process at the trapping stage are similar to invasion percolation with trapping, which belongs to the same universality class as ordinary percolation (Dias and Wilkinson [23]). However the allowance for the partial saturation state of a given region introduces certain novel aspects. The process can be dicectly simulated by an invasion percolation algorithm. The invasion rules are as follows: First, a permeability distribution is randomly assigned to individual blocks. Subsequently, from one face of the sample, invasion takes place, the progress of which is controlled by incrementally decreasing $k_{d}$ (from $k_{\max }$ to $k_{\min }$ ), or equivalently by increasing the capillary pressure. A block is allowed to drain provided that its percolation threshold is exceeded (has $k>k_{d}$ ), it is accessible to the inlet face by $a_{\text {in }}$ continuous path and it is not blocked. After each incremental decrease in $k_{d}$, the saturation content of all blocks that have been invaded is updated according to (77). Trapped regions, if any, are not allowed to drain further. The process continues until $k_{d}=k_{\min } / \alpha$ (Figure 56). One should note that due to the partial staturation sates and the need to update the saturation of each region, the above rules are different from the standard Invasion Percolation algorithm (Feder [28]).

The occupancy of the various regions at three sequential stages of the process is illustrated in Figures $57 \mathrm{a}, 57 \mathrm{~b}$, and 57c. A uniform distribution of $\sqrt{k}$ with a linear $J\left(S_{w}\right)$ function and $S_{w r}=0$ were taken in these 2-D simulations. In the illustrations, lighter regions correspond to higher $S_{w}$ values, while black indicates fully drained blocks $\left(S_{w}=0\right)$. The drainage occurs in the direction from bottom to top, the lateral boundaries being impermeable to flow. As is apparent, drainage is strongly influenced by accessibility, in addition to the individual capillary pressure characteristics. In particular, large scale trapping is obtained when completely drained (black) regions block partially drained blocks (Figure 57c). For the particular conditions of Figure 57, this large scale residual saturation is 0.1275 . The corresponding capillary pressure curve obtained by the same algorithm tor the same distributions but for a cubic 3.D lattice is shown in Figure 58 for 


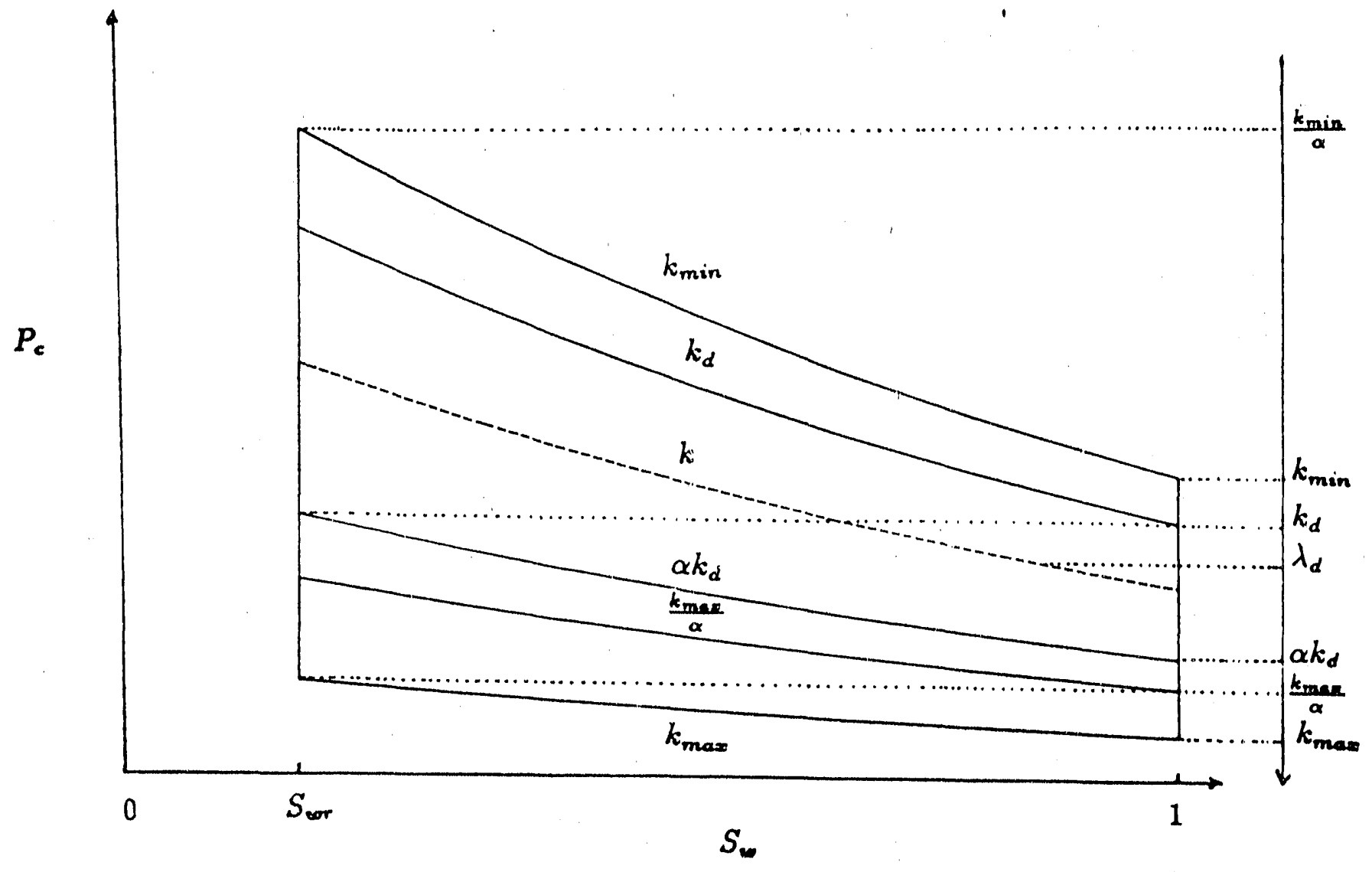

Figure 56: Schematic indicating notation used. 
different values of the parameter $\alpha$. The location of the large scale capillary pressure curve vis-avis the curves corresponding to the minimum and maximum permeabilities in the sample (straight lines in Figure 58) are noted. The general features of such large scale curve are indicative of a percolation process. Percolation thresholds for invasion or trapping at the two ends of the large scale $\leq$ sturation. should be noted. As illustrated above, large scale averaging induces large scale trapping that leads to a non-«ero large scale residual saturation $\left\langle S_{w}\right\rangle_{r}$, different than the local value $S_{w r},($ taken as zero in the simulations of Figures 57 and 58). This non-trivial result is discussed more fully in a later section.

Because of the percolation aspects, the large scale capillary pressure curve is not amenable to an analytic description. This is to be contrasted to classical averaging schemes, where effective coefficients are to be obtained from the solution of boundary value problems in representative cells. However, while an explicit description is not possible, the behavior near the two percolation thresholds can be analysed by ordinary percolation theory (near invasion) and by ordinary percolation with trapping (near trapping). This is discussed in the next sections.

\subsection{BEHAVIOR NEAR INVASION THRESHOLD}

Near invasion, ordinary percolation theory can be directy applied to yield the condition

$$
\int_{P_{c d, \text { adin }}}^{\left\langle P_{c}\right\rangle_{d}} F\left(P_{c d}\right) d P_{c d}=p_{c}
$$

where subscript min is self-evident, and $p_{c}$ is the percolation threshold of the heterogeneous medium. Equivalently, one may define a permeability threshold $k_{c}$ for large scale penetration,

$$
\left\langle P_{c}\right\rangle_{d}=\frac{\gamma}{\sqrt{k_{c}}} J(1)
$$

which, in view of $(75)$, satisfies

$$
\int_{k_{c}}^{k_{\max }} f(k) d k=p_{c}
$$

Parameters, such as percolation threshold, should correspond to percolation in continua. However, use is made below of results from discrete approximations and site percolation, corresponding to square or cubic lattices. Invasion thresholds are evident in Figure 58. 


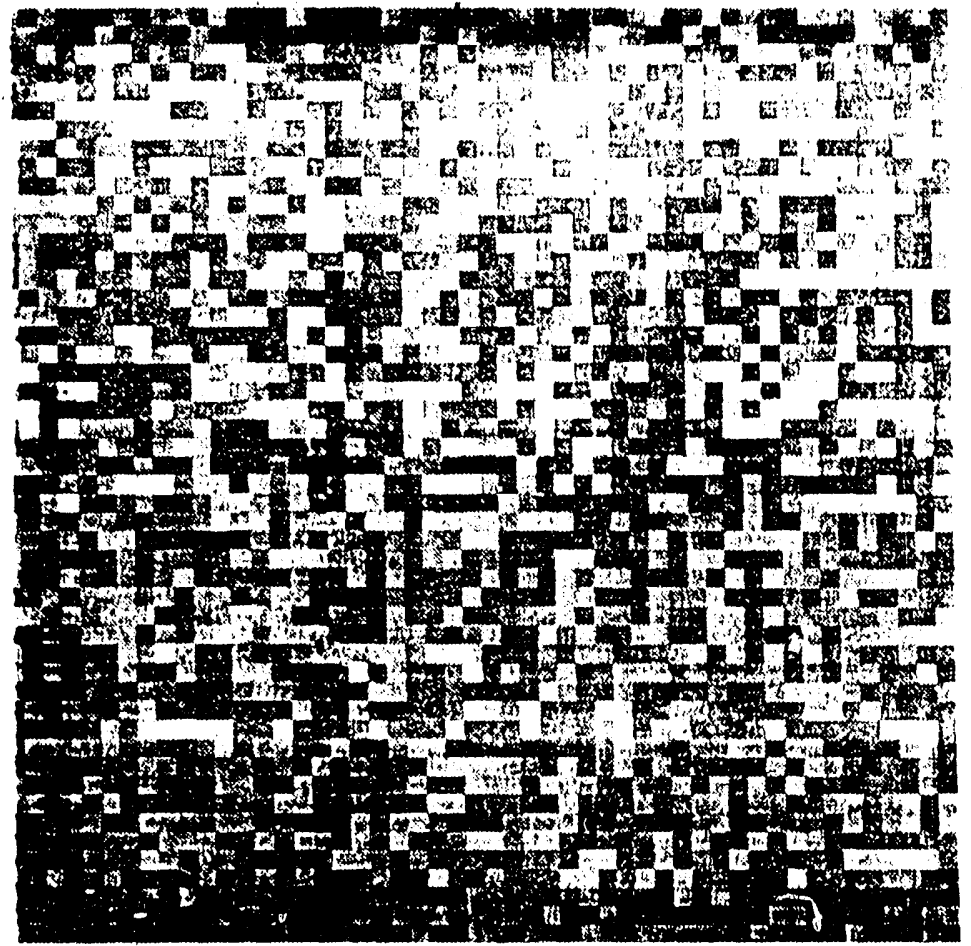

(a)

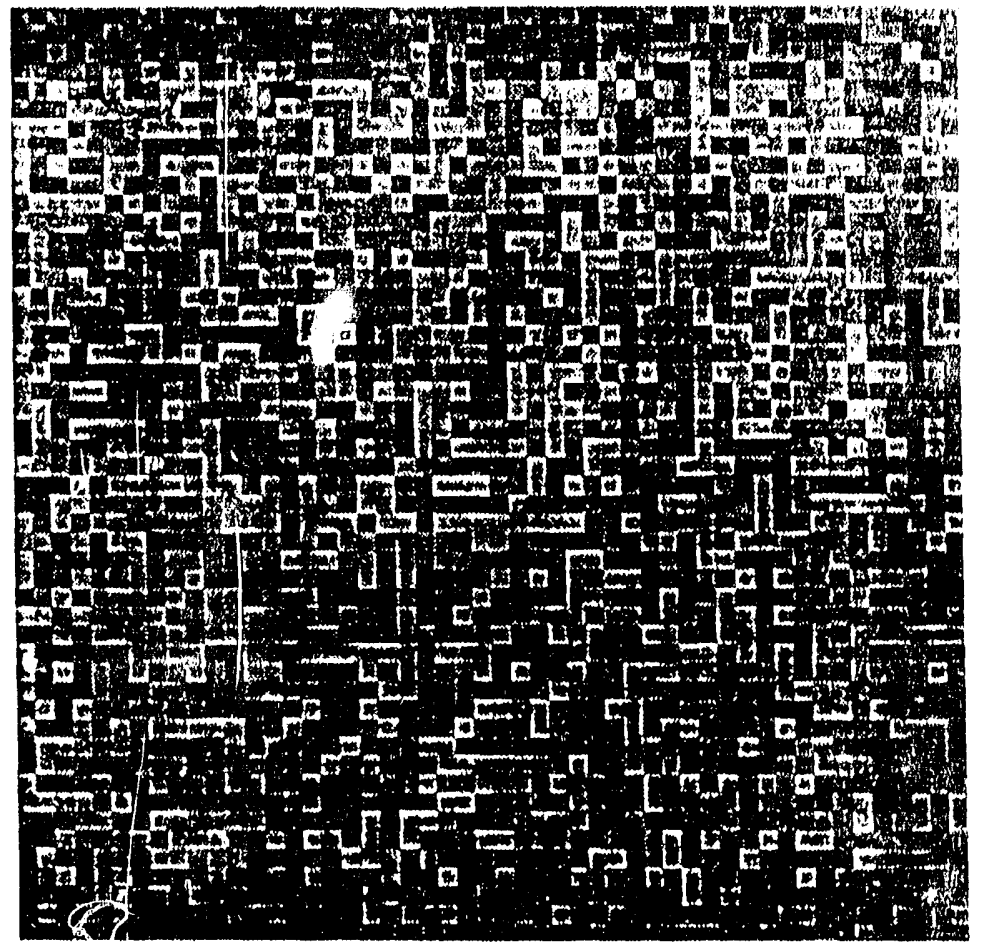

(b)

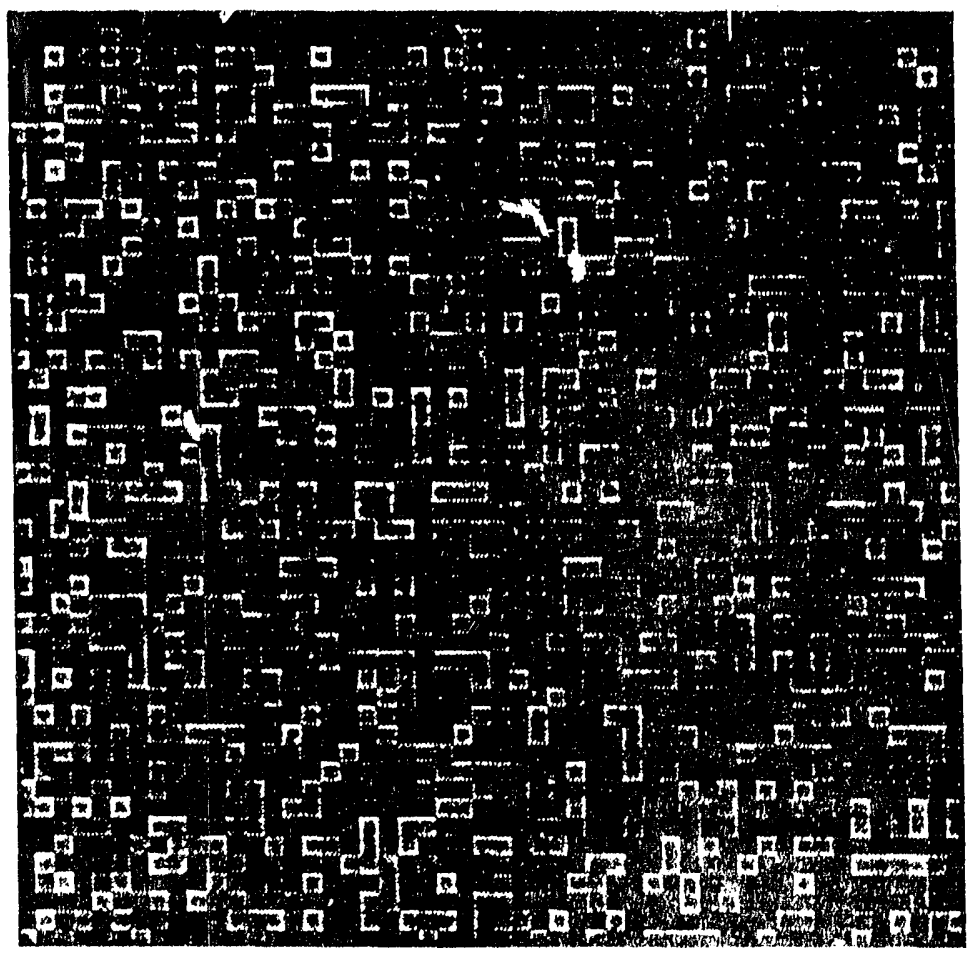

(c)

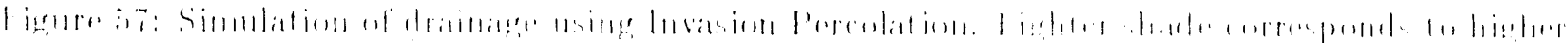
Valle. 


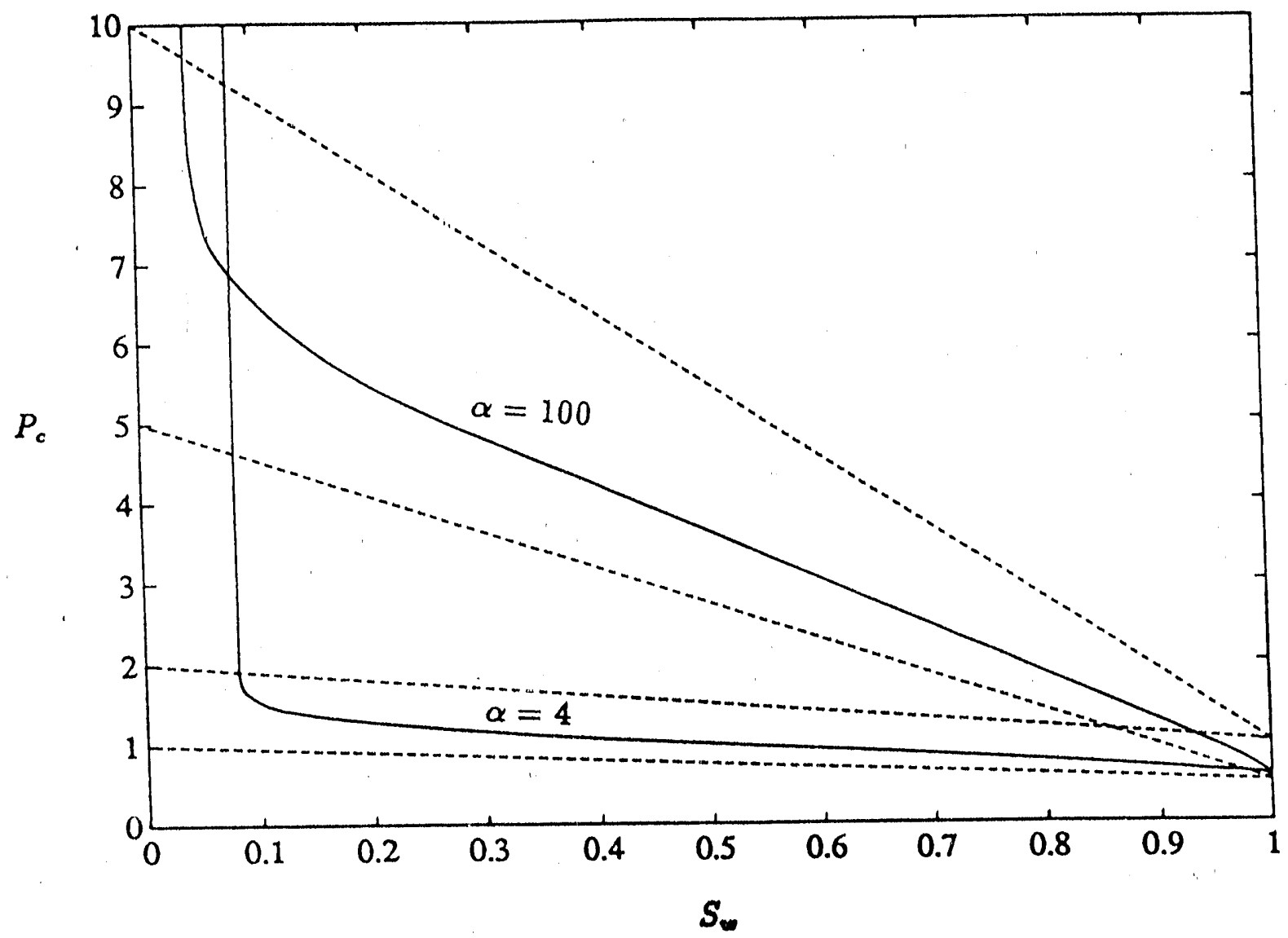

Figure 5is: Large scale capillary pressure curves obtained by Invasion Percolation in a 3-D cubic lattice. 
For the saturation dependence near this threshold the following applies. Given a capillary pressure exceeding (82), large scale penetration by the non-wetting phases occurs in the accessible regions only (those belonging to the percolation cluster), the volume fraction, $X_{a}$, of which scales with the distance from the percolation threshold

$$
X_{a} \sim\left[\int_{k_{d}}^{k_{c}} f(k) d k\right]^{\beta}
$$

where $\beta$ is a universal exponent, equal to 0.4 for 3-D (Stauffer, [87]). More generally, as long as $k_{d} \geq \frac{k_{\text {man }}}{\alpha}$, which ensures that trapping has not as yet occured, since complete draining has not been reached in any region, we have

$$
\begin{aligned}
X_{a} & =X_{0}(p) \\
p & =\int_{h_{d}}^{k_{\max }} f(k) d k
\end{aligned}
$$

where $X$, is the percolation probability of ordinary site percolation. Upon onset of drainage, nonwetting phase invasion occurs only in regions with $k_{d} \leq k$, of volume fraction $X_{o}(p)$. The volume distribution of this set is

$$
f_{a}\left(k ; k_{d}\right)= \begin{cases}0 & ; k<k_{d} \\ \frac{t(k)}{p} & ; k_{d}<k<k_{\max }\end{cases}
$$

each element of which has the following value of wetting saturation

$$
S_{w}=I\left(\frac{k}{k_{d}}\right)
$$

The large scale saturation $\left\langle S_{w}\right\rangle$ is the sum of the individual contributions

$$
\left\langle S_{w}\right\rangle=1-X_{d}(p)+\frac{X_{s}(p)}{p} \int_{k_{d}}^{k_{\text {max }}} f(k) I\left(\frac{k}{k_{d}}\right) d k
$$

The large scale capillary pressure curve in this regime follows by combining (76),(78),(86) and (88). The validity of this result is restricted to $k_{d} \geq \frac{k_{\text {max }}}{\alpha}$. For highar capillary pressures, certain invaded regions may completely drain $\left(S_{w}=S_{w r}\right)$, in which case they can act to block neighboring elements from further drainage (since hydraulic connectivity is lost). Such trapping behavior can be analysed by a modification of ordinary percolation to account for trapping. 


\subsection{BEHAVIOR NEAR TRAPPING THRESHOLD}

Ordinary percolation with trapping was proposed by Dias and Wilkinson [23] as an alternative description of invasion percolation near the trapping threshold. In this method, the displacing phase is assumed to invade any allowed region (with permeability $k>k_{d}$ ) as long as it is not blocked, but independently of whether the in rading phase is connected or not. Clearly, this assumption restricts the method to regions away from the invasion threshold. Independently, Yortsos and Sharma [106] proposed essentially the same method, although in a different context involving pore plugging induced a by gas-solid reaction in porous particles. Due to the nature of that problem, which does not require invasion thresholds, the latter application is exact throughout. Their formalism will be also employed in the present paper.

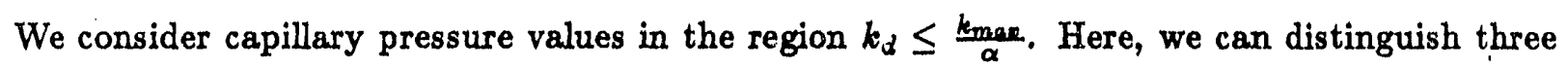
sets in the sample (Figure 59):

(i) Set $a$ consisting of regions (denoted by subscript $a$ ) that are either actively draining or they are not yet invaded because of higher threshold;

(ii) Set $b$ consisting of regions (denoted by subscript $b$ ) that have completely drained, thus they may block the drainage of surrounding regions; and

(iii) Set $c$ consisting of regions (denoted by subscript $n a$ ) that did not complete their drainage due to trapping.

To evaluate the statistics of each set (volume fraction $p d f$ and saturation content) we proceed as follows.

The first set is the sample-spanning (infinite) cluster of regions that result from the process of disconnection, brought about by regions that drained completely. Since the original permeability distribution is assumed random and uncorrelated we have

$$
X_{a}=X_{0}(q)
$$

where $q$ (Figure 54b) is the fraction of regions of permeability low enough so that they can not fully drain 


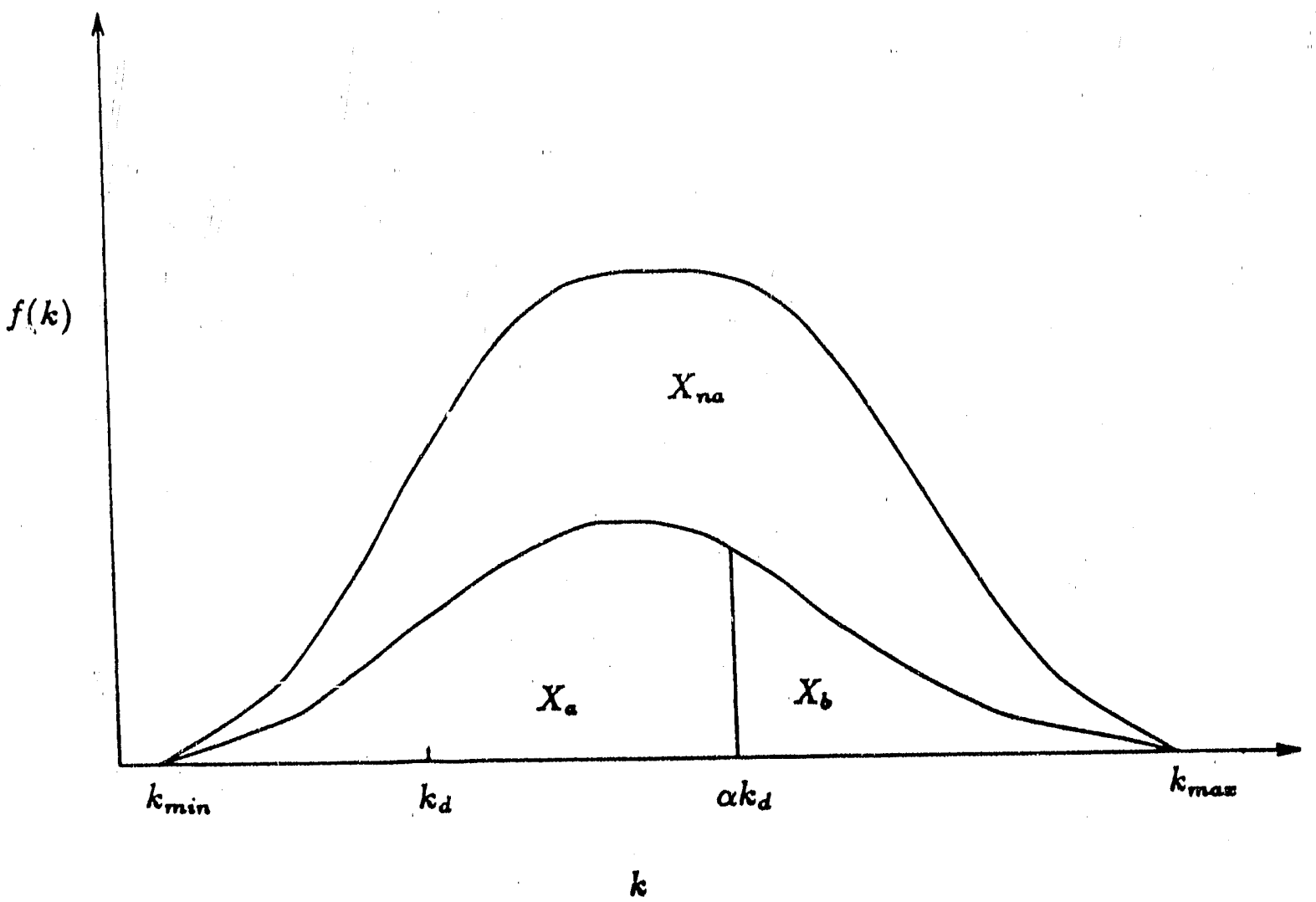

Figure 59: Schematic illustrating the three sets $(a, b)$ and $(n a)$. 


$$
q=\int_{k_{\min }}^{\alpha k_{d}} f(k) d k \quad ; \quad k_{d}<\frac{k_{\max }}{\alpha}
$$

The pdf of this set is

$$
f_{\alpha}\left(k ; k_{d}\right)= \begin{cases}0 & ; k>\alpha k_{d} \\ \frac{f(k)}{q} & ; k_{\min }<k \leq \alpha k_{d}\end{cases}
$$

while the saturation content of each region of the set is $S_{w}=I\left(\frac{k}{k_{d}}\right)$, as described in (87) above (Figure 59).

The second set contains regions that have drained completely. To obtain its number density we appiy a population balance following the approach of Yortsos and Sharma [107]. During the incremental change in capillary pressure, or equivalently the change $-\Delta k_{d}$ in the variable $k_{d}$, all regions of set $(a)$ with permeability values in the range $\alpha\left(k_{d}-\Delta k_{d}\right)<k<\alpha k_{d}$ drain completely, thus they transfer to set (b). Since this occurs randomly, we have,

$$
\Delta X_{b}=-\alpha X_{s}(q) f_{a}\left(\alpha k_{d} ; k_{d}\right) \Delta k_{d}
$$

thus,

$$
\frac{d X_{b}}{d k_{d}}=-\alpha X_{a}(q) f_{a}\left(\alpha k_{d} ; k_{d}\right)
$$

Combining the above with (90) and integrating yields

$$
X_{b}(q)=\int_{q}^{1} \frac{X_{s}(q) d q}{q}
$$

a result also obtained by Dias and Wilkinson [23]. (One should also recall that the problem reduces to the classical percolation with trapping when $\alpha=1$ ). The $p d f$ of set (b) is readily obtained from above

$$
f_{b}\left(k ; k_{d}\right)= \begin{cases}0 & ; k<\alpha k_{d} \\ \frac{X_{d}(q t) f(k)}{q\left(X_{b}(q)\right.} & ; \alpha k_{d} \leq k\end{cases}
$$

where

$$
q \prime=\int_{k_{\min }}^{k} f(k) d k \quad ; \quad \alpha k_{d} \leq k
$$


It should be noted that this set contains regions with sufficiently large permeability (larger then $\alpha k_{d}$ ) (see Figure 59). By definition, their saturation content is uniform, equal to $S_{w r}$.

Finally, the third set is the complement of the other two

$$
X_{n a}=1-X_{a}-X_{b}
$$

Its $p d f$ can be obtained from a population balance, either directly from the previous sets, or by integrating tine equivalent equation

$$
\frac{\partial}{\partial k_{d}}\left[X_{n a} f_{n a}\right]=\frac{d X_{n a}}{d k_{d}} f_{a}\left(k ; k_{d}\right)
$$

where $X_{n a}$ and $f_{a}$ are given by (97) and (91), respectively. The final result is

$$
f_{n a}\left(k ; k_{d}\right)= \begin{cases}\frac{f(k)}{X_{n a}(q)}\left[1-\frac{X_{d}(q)}{q \prime}\right] ; & \alpha k_{d}<k \\ \frac{f(k)}{X_{n a}(q)}\left[1-\frac{X_{0}(q)}{q}\right] ; & k<\alpha k_{d}\end{cases}
$$

The correspondirig saturation of each region in this set is evaluated as follows. Before becoming trapped (an event occurring at stage $\lambda_{d} \geq k_{d}$ ) a region was allowed to drain freely, provided that it has sufficiently high permeability $\left(k>\lambda_{d}\right)$. Upon trapping, the element cannot drain further, its saturation being fixed to ine value $S_{w}=I\left(\frac{k}{\lambda_{d}}\right)$. The reader may consult Figure 56 for the notation used. The saturation content of all regions in the set with permeability value $k$ can be computed from knowledge of the trapping rate of such regions, $t\left(k ; \lambda_{d}\right)$. From $(29)$, the latter is

$$
t\left(k ; \lambda_{d}\right)=\left.\left(\frac{d X_{n a}}{d k_{d}} f_{a}\left(k ; k_{d}\right)\right)\right|_{k_{d}=\lambda_{s}}
$$

and using (99),

$$
t\left(k ; \lambda_{d}\right)= \begin{cases}0 & ; \alpha \lambda_{d}<k \\ f(k) \frac{d}{d \lambda_{d}}\left[\frac{x \cdot(q)}{q}\right] & ; k<\alpha \lambda_{d}\end{cases}
$$

where, $q$ above is implied to be a function of $\lambda_{d}$. The saturation of regions with permeability $k$ is, therefore,

$$
S_{n a}\left(k ; k_{d}\right)=\frac{1}{X_{n a} f_{n a}\left(k ; k_{d}\right)} \int_{k_{d}}^{\frac{n_{\text {max }}}{a}} t\left(k ; \lambda_{, d}\right) I\left(\frac{k}{\lambda_{d}}\right) d \lambda_{d}
$$

By combining the above, the large scale average saturation is 


$$
\begin{array}{r}
\left\langle S_{w}\right\rangle=X_{a}(q) \int_{k_{\text {min }}}^{k_{\text {max }}} f_{a}\left(k ; k_{d}\right) I\left(\frac{k}{k_{d}}\right) d k+X_{b}(q) S_{w r}+ \\
\int_{k_{\text {min }}}^{k_{\text {max }}} \int_{k_{d}}^{\frac{k_{\text {max }}}{\alpha}} t\left(k ; \lambda_{d}\right) I\left(\frac{k}{\lambda_{d}}\right) d \lambda_{d} d k
\end{array}
$$

which further simplifies to

$$
\begin{array}{r}
\left\langle S_{w}\right\rangle=S_{w r}+X_{\alpha}(q) \int_{k_{\min }}^{k_{\text {max }}} f_{\alpha}\left(k ; k_{d}\right)\left(I\left(\frac{k}{k_{d}}\right)-S_{w_{r}}\right) d k+ \\
\int_{k_{\text {min }}}^{k_{\text {max }}} \int_{k_{d}}^{\frac{k_{\text {max }}}{\alpha}} t\left(k ; \lambda_{d}\right)\left(I\left(\frac{k}{\lambda_{d}}\right)-S_{\text {ivr }}\right) d \lambda_{d} d k
\end{array}
$$

so that we may effectively consider $S_{w r}$ to be zero. This simplification will be used in the numerical illustrations below. A final rearrangement leads to

$$
\begin{array}{r}
\left\langle S_{w}\right\rangle=\frac{X_{s}(q)}{q} \int_{k_{\min }}^{k_{\text {max }}} f(k) H\left(\alpha k_{d}-k\right) I\left(\frac{k}{k_{d}}\right) d k+ \\
\int_{k_{\min }}^{\alpha k_{d}} f(k) \int_{k_{d}}^{\frac{k_{\max }}{\alpha}} \frac{\partial}{\partial \lambda_{d}}\left[\frac{X_{s}(q)}{q}\right] I\left(\frac{k}{\lambda_{d}}\right) d \lambda_{d} d k+ \\
\int_{\alpha k_{d}}^{k_{\text {max }}} f(k) \int_{\frac{k}{\alpha}}^{\frac{k_{\text {max }}}{\alpha}} \frac{\partial}{\partial \lambda_{d}}\left[\frac{X_{s}(q)}{q}\right] I\left(\frac{k}{\lambda_{d}}\right) d \lambda_{d} d k
\end{array}
$$

where $H(z)$ is the step function and use was made of (91). As easily varified for $\alpha=1$, whence $I=1$, the above reduces to the expression for percolation with trapping (Dias and Wilkinson [23])

$$
\left\langle S_{w}\right\rangle=1-\int_{q}^{1} \frac{X_{s}(q) d q}{q}
$$

\subsection{RESULTS}

Equations (104) or (105) give the desired large scale saturation average as a single function of the variable $k_{d}$, which parametrizes the capillary pressure via (76). Variables $X_{a}, X_{b}, f_{a}, t$ and $I$ have been explicity defined above. We may note the following:

1. Equation (105) may be formally extended towards the invasion threshold, $\frac{k_{\text {max }}}{\alpha}<k_{d}$, where now $q=1, X_{b}=0, t=0, X_{a}=1$, thus 


$$
\left\langle S_{w}\right\rangle=\int_{k_{\min }}^{k_{\text {max }}} f(k) I\left(\frac{k}{k_{d}}\right) d k
$$

However, in this result we have ignored the invading phase connectivity (assumption $X_{0}(p)=1$ ), compare also with (88)).

2. It is evident that the drainage process terminates when set $(a)$ becomes disconnected. This occurs at the value $k_{d}^{*}$,

$$
\int_{k_{\min }}^{\alpha k_{d}^{*}} f(k) d k=p_{c}
$$

where $p_{c}$ is the percolation threshold. For a given permeability field, $f(k)$, in the limit of large $\alpha$, we have $k_{d}^{*} \sim O\left(\frac{1}{\alpha}\right), P_{c}^{*} \sim O(\alpha)$. Note, however, that $k_{d}^{*} \geq \frac{k_{\min }}{\alpha}$. This implies that the process terminates before complete drainage of the tight regions.

3. The large scale residual saturation is

$$
\left\langle S_{w}\right\rangle_{r}=S_{w r}+\int_{h_{\min }}^{k_{\text {max }}} \int_{k_{d}^{*}}^{\frac{h_{\text {max }}}{\alpha}} t\left(k ; \lambda_{d}\right)\left(I\left(\frac{k}{\lambda_{d}}\right)-S_{w r}\right) d \lambda_{d} d k
$$

Since the integral above is positive, it represents the contribution to the residual saturation from large scale trapping. This result is consistent with the numerical simulation of Figures 57 and 58 , and represents a key finding of the present work. As expected, for $\alpha=1, S_{w r}=0$, whence $I(x)=1$, the residual is identical to the value of percolation with trapping (Dias and Wilkinson [23])

$$
\left\langle S_{w}\right\rangle_{r}=1-\int_{p_{c}}^{1} \frac{X_{s}(q) d q}{q} \sim\left\{\begin{array}{l}
0.60 \text { for } 2-\mathrm{D} \text { square } \\
0.34 \text { for } 3-\mathrm{D} \text { cubic }
\end{array}\right.
$$

4. Large scale trapping decreases as $\alpha$ increases, or as the individual capillary pressure curves become steeper. However, there is a limiting, non-zero trapped saturation no matter how large $\alpha$ is. This can be seen by first noting that $I(x)$ can be approximated at large $\alpha$ by a function $F$ of a single argument,

$$
I(x) \simeq F\left(\frac{\alpha-x}{\alpha-1}\right) \quad 1<x<\alpha
$$

From (109), the residual value is 


$$
\begin{aligned}
\left\langle S_{w}\right\rangle_{r} \rightarrow & \int_{k_{\text {min }}}^{\alpha k_{d}^{*}} f(k) \int_{k_{d}^{*}}^{\frac{k_{\text {max }}}{\alpha}} \frac{\partial}{\partial \lambda_{d}}\left[\frac{X_{d}(q)}{q}\right] F\left(\frac{\alpha-\frac{k}{\lambda_{d}}}{\alpha-1}\right) d \lambda_{d} d k+ \\
& +\int_{\alpha k_{d}^{*}}^{k_{\text {maxa }}} f(k) \int_{\frac{k}{\alpha}}^{\frac{k_{\max }}{\alpha}} \frac{\partial}{\partial \lambda_{d}}\left[\frac{X_{s}(q)}{q}\right] F\left(\frac{\alpha-\frac{k}{\lambda_{d}}}{c-1}\right) d \lambda_{d} d k
\end{aligned}
$$

where use is made of (101) at $q=p_{c}$. Subsequent change of variables, $\alpha \lambda_{d}=\xi$, gives the following, where it is recalled from (108) that $\alpha k_{d}^{*}$ is a constant (denoted by $\kappa$ below),

$$
\begin{aligned}
\left\langle S_{w}\right\rangle_{r} & \rightarrow \int_{k_{\text {min }}}^{\kappa} f(k) \int_{\kappa}^{k_{\text {max }}} \frac{\partial}{\partial \xi}\left[\frac{X_{0}(q)}{q}\right] F\left(1-\frac{k}{\xi}\right) d \xi d k \\
& +\int_{\kappa}^{k_{\text {max }}} f(k) \int_{k}^{k_{\text {max }}} \frac{\partial}{\partial \xi}\left[\frac{X_{0}(q)}{q}\right] F\left(1-\frac{k}{\xi}\right) d \xi d k
\end{aligned}
$$

This value is independent of $\alpha$. This rather remarkable result is verified in the numerical illustrations of Figures 58 and 60 .

\subsection{EXAMPLES}

We demonstrate the above by the full numerical simulation of the process on $2-D$ (square) and 3 - D (cubic) lattices. As before, we take the function $I(x)$ to be linear in $\sqrt{x}$ (straight line Leverett $J$ function) and the distribution of $\sqrt{k}$ to be uniform. Invasion percolation and percolation with tr spping, subject to the new trapping rules, were simulated. Typical results for the first case $w$ are shown in Figures 57 and 58. Results of percolation with trapping are shown in Figures 60a anc $60 \mathrm{~b}$, for $2-\mathrm{D}$ and $3-\mathrm{D}$ lattices, respectively. The large scale invasion and trapping thresholds, discussed above,were found to be in excellent agreement with the numerical results. In addition, the existence of large scale trapping and its dependence on the parameter $\alpha$ are clearly in evidence in both Figures 58 and 60 .

For a simplified, but explicit, representation, a bimodal permeability distribution was also considered (Figure 61)

$$
f(k)=\rho \delta\left(k-k_{1}\right)+(1-\rho) \delta\left(k-k_{2}\right) \quad ; \quad k_{1}<k_{2}, \quad 0<\rho<1
$$

where, we have assumed $k_{1}<\frac{k_{2}}{\alpha}$. For this system, the process occurs in two stages: 


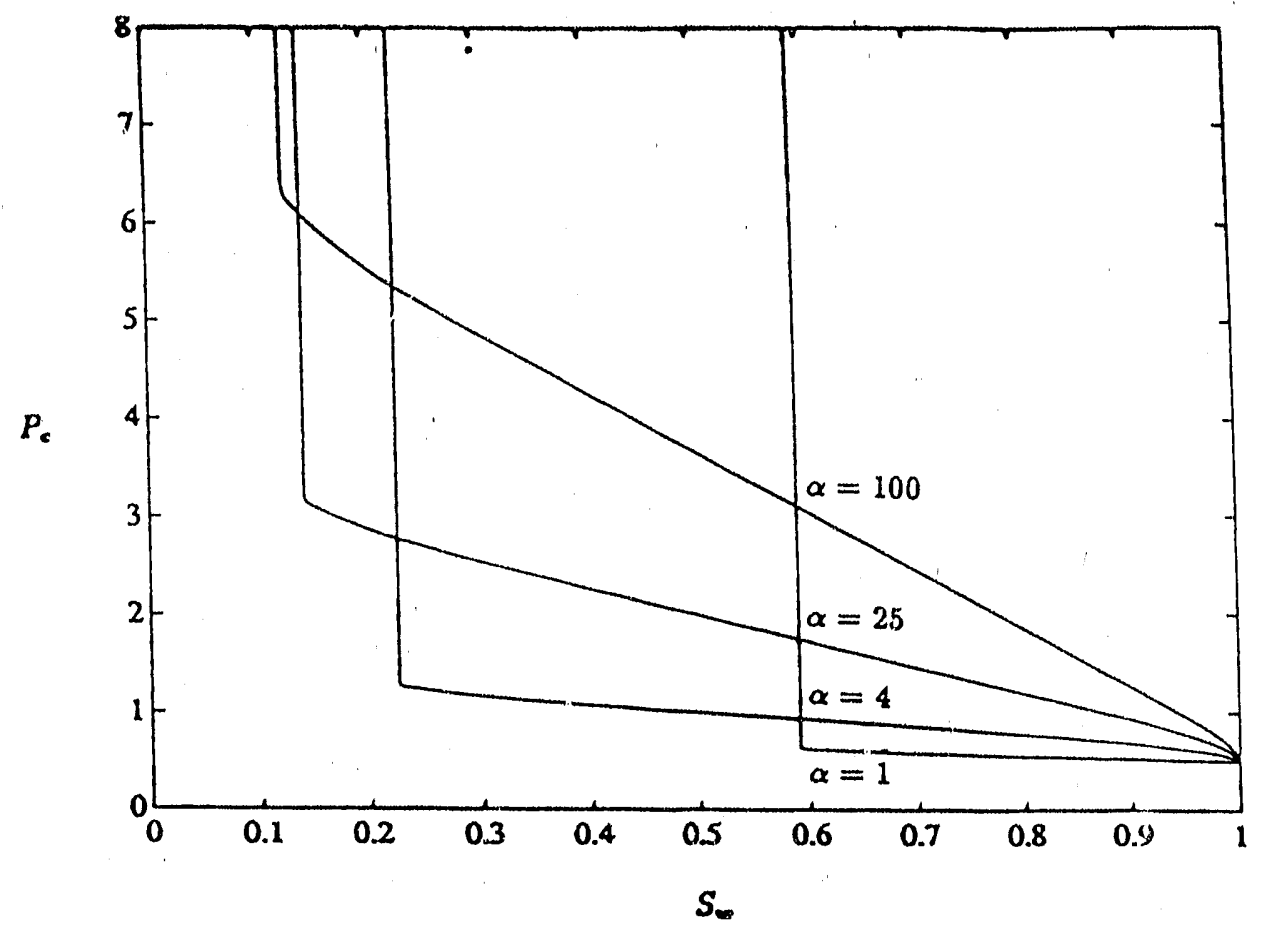

(a)

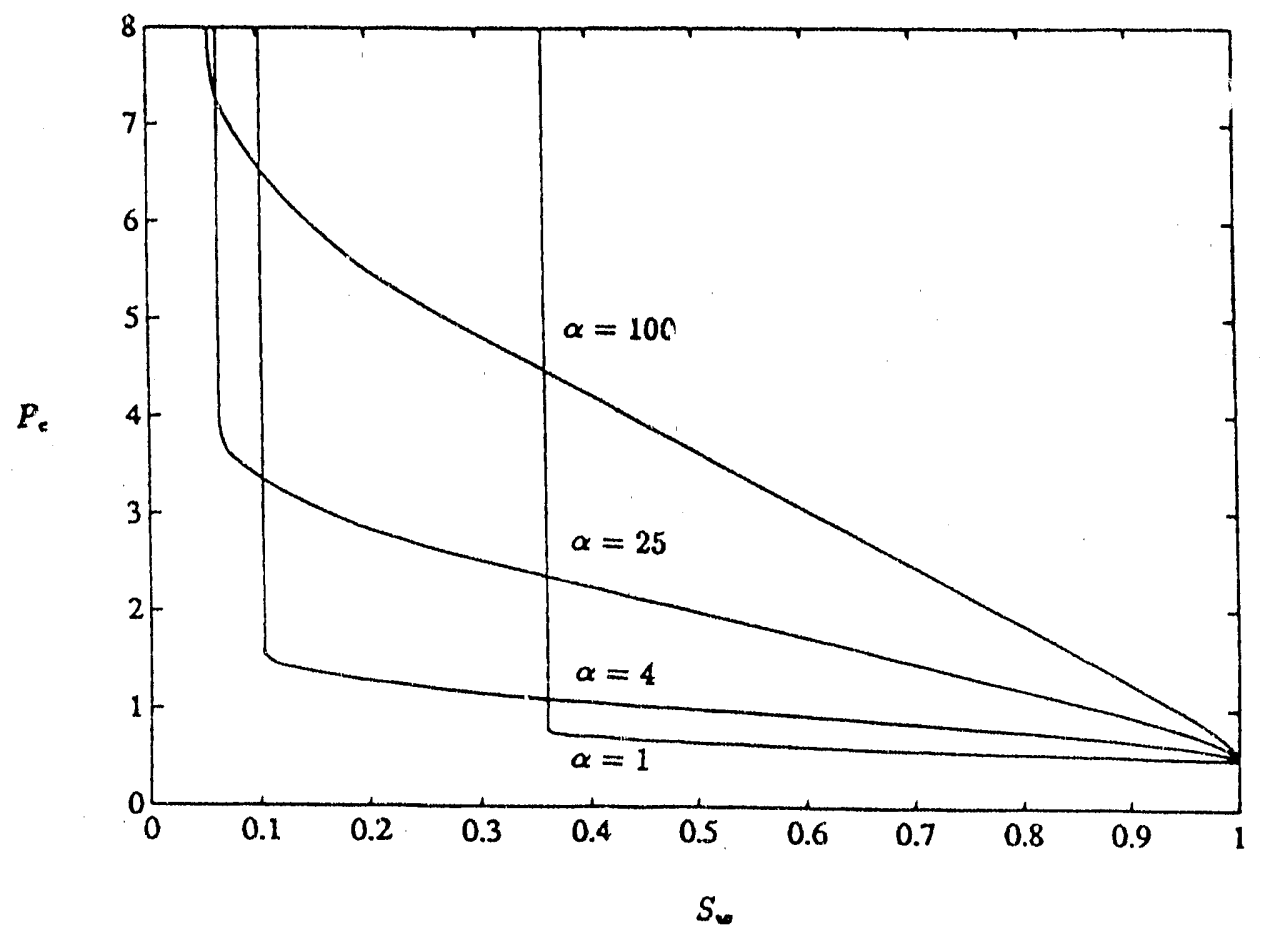

(b)

Figure 60: Large scale capillary pressure curves obtained by percolation with trapping. 


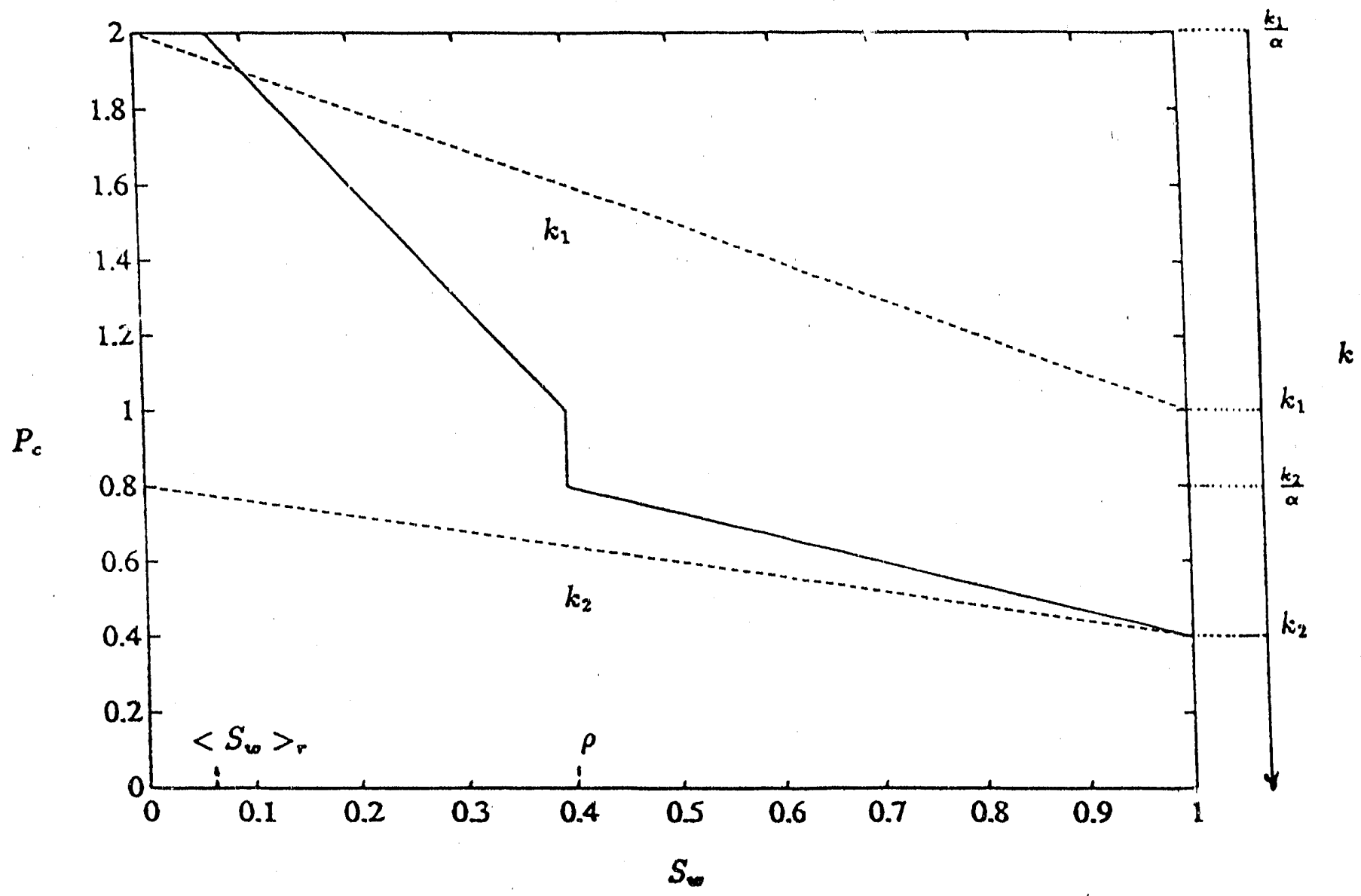

Figure 61: Large scale capillary pressure curve for a bimodal distribution, $k_{1}=1, k_{2}=6.25, \rho=0.4$ and $\alpha=4$. 
Stage $1\left(k_{1}<\frac{k_{2}}{\alpha}<k_{d}<k_{2}\right)$ is described by penetration of the regions with high permeability $\left(k_{2}\right)$, by unrestricted drainage and by no trapping. The saturation contribution is from set $(a)$ only $(q=1$ in (105)):

$$
\left\langle S_{w}\right\rangle=\rho+(1-\rho) I\left(\frac{k_{2}}{k_{d}}\right)
$$

Regions with permeability $k_{1}$ have not been invaded, their saturation remaining constant (first term on the $R H S$ ). Assuming a linear $J$ function (so that $I(x)=\frac{1}{\sqrt{\alpha-1}}(\sqrt{\alpha}-\sqrt{x}$ ) we may rearrange (115) to

$$
\left\langle S_{w}\right\rangle=\rho+\left(\frac{1-\rho}{\sqrt{\alpha}-1}\right)\left(\sqrt{\alpha}-P_{c} \sqrt{k_{2}}\right) \quad ; \quad P_{c}<\frac{\sqrt{\alpha}}{\sqrt{k_{2}}}
$$

Like the individual capillary pressure curves of each region (Figure 61), the large scale capillary pressure curve is also a straight line, although with a higher absolute slope. This stage terminates when $k_{d}=\frac{k_{2}}{\alpha}$, at which time all regions with value $k_{2}$ have completely drained and $\left\langle S_{w}\right\rangle=\rho$. Stage $2\left(k_{d}<\frac{k_{2}}{\alpha}\right)$ involves two steps, one during which there is no change in saturation, $k_{1}<k_{d}$, and another in which the low permeability regions drain, $k_{d}<k_{1}$. The process terminates at $k_{d}^{*}=\frac{k_{1}}{\alpha}(+\epsilon)$, where the trapping threshoid is reached. During the first step, $k_{1}<k_{d}<\frac{k_{2}}{\alpha}$, the contribution from set $(a)$ is $X_{d}(q) I\left(\frac{k_{1}}{k_{d}}\right)=X_{d}(q)$ (first term on $R H S$ of (105)), while that of set $(n a)$ is $q\left[1-\frac{X_{0}(q)}{q}\right]$ (second term on $R H S$ of (105)). But $q=\rho$, thus

$$
\left\langle S_{w}\right\rangle=\rho \quad ; \quad \frac{\sqrt{\alpha}}{\sqrt{k_{2}}}<P_{c}<\frac{1}{\sqrt{k_{1}}}
$$

As expected, the large-scale average is at a constant value until regions with permeability $k_{1}$ start draining (Figure 61). During the second step, $\frac{k_{1}}{\alpha} \leq k_{d}<k_{1}$, the contribution from set $(a)$ is $X_{s}(\rho) I\left(\frac{k_{1}}{k_{d}}\right)$, and from set $(n a)$ is

$$
\rho\left[1-\frac{X_{s}(\rho)}{\rho}\right]+\rho \int_{k_{d}}^{k_{1}} \frac{\partial}{\partial \lambda_{d}}\left[\frac{X_{s}}{q}\right] I\left(\frac{k_{1}}{\lambda_{d}}\right) d \lambda_{d}
$$

Using integration by parts the above integral is shown to be zero. Thus,

$$
\left\langle S_{w}\right\rangle=\rho-X_{d}(\rho)\left[1-I\left(\frac{k_{1}}{k_{d}}\right)\right] \quad ; \quad \frac{k_{1}}{\alpha}<k_{d}<k_{1}
$$

and, for our particular case, 


$$
\left\langle S_{w}\right\rangle=\rho-\frac{X_{a}(\rho)}{(\sqrt{\alpha}-1)}\left[\sqrt{k_{1}} P_{c}-1\right] \quad ; \quad \frac{1}{\sqrt{k_{1}}}<P_{c}<\frac{\sqrt{\alpha}}{\sqrt{k_{1}}}
$$

This is also a straight-line segment but with absolute slope larger than that of the individual elements (Figure 61). As indicated previously, the process terminates at $P_{c}=\frac{\sqrt{\alpha}}{\sqrt{k_{1}}}$, where the large scale residual saturation is

$$
\left\langle S_{w}\right\rangle_{r}=\rho-X_{s}(\rho)
$$

This value is substantial if $\rho$ is not too far from the percolation threshold $p_{c}$. The difference $\rho-X_{\odot}(\rho)$ is plotted in Figure 62 for both square and cubic lattices.

One concludes that large scale capillary pressure functions have a non-trivial, certainly nonlinear, dependence that reflects percolation and tripping characteristics at two different scales of disorder, the microscopic (pore structure) and the macroscopic (permeability field). As long as the dimensionality of these two fields is the same, the scaling behavior near the thresholds is also the same for either individual capillary curves (namely the $J\left(S_{w}\right)$ curve) or the large scale average. Otherwise, scaling exponents and percolation characteristics corresponding to the particular lattice dimension should apply. Such properties are reviewed in Stauffer [86] (see also Sahimi and Yortsos [75]). It must be pointed out that in many real systems, permeability fields are likely to be 2-D rather than $3-D$, in which case the large scale trapping predicted here would be relatively extensive (compare Figure 62). We should also reiterate that the above approach applies for spatially uncorrelated permeability fields, although it can be extended to fields with small, but finite correlation lengths.

\subsection{FLOW PERMEABMITHES}

Having determined the large scale fluid phase partition under local capillary control, we may next proceed with the derivation of effective flow permeabilities. Single-phase permeabilities can be averaged by a variety of methods, for instance following any of the classical approaches (e.g. Whitaker [98]). It is also worth pointing out that large scale averaging can be performed with the techniques of pore level modeling. For example, we can use Effective Medium Theory (EMA) away from percolation and percolation scaling laws near thresholds. Advances and applications are reviewed in Katz and Thompson [40], Sharma and Yortsos [83] and Sahimi et al. [74]. 


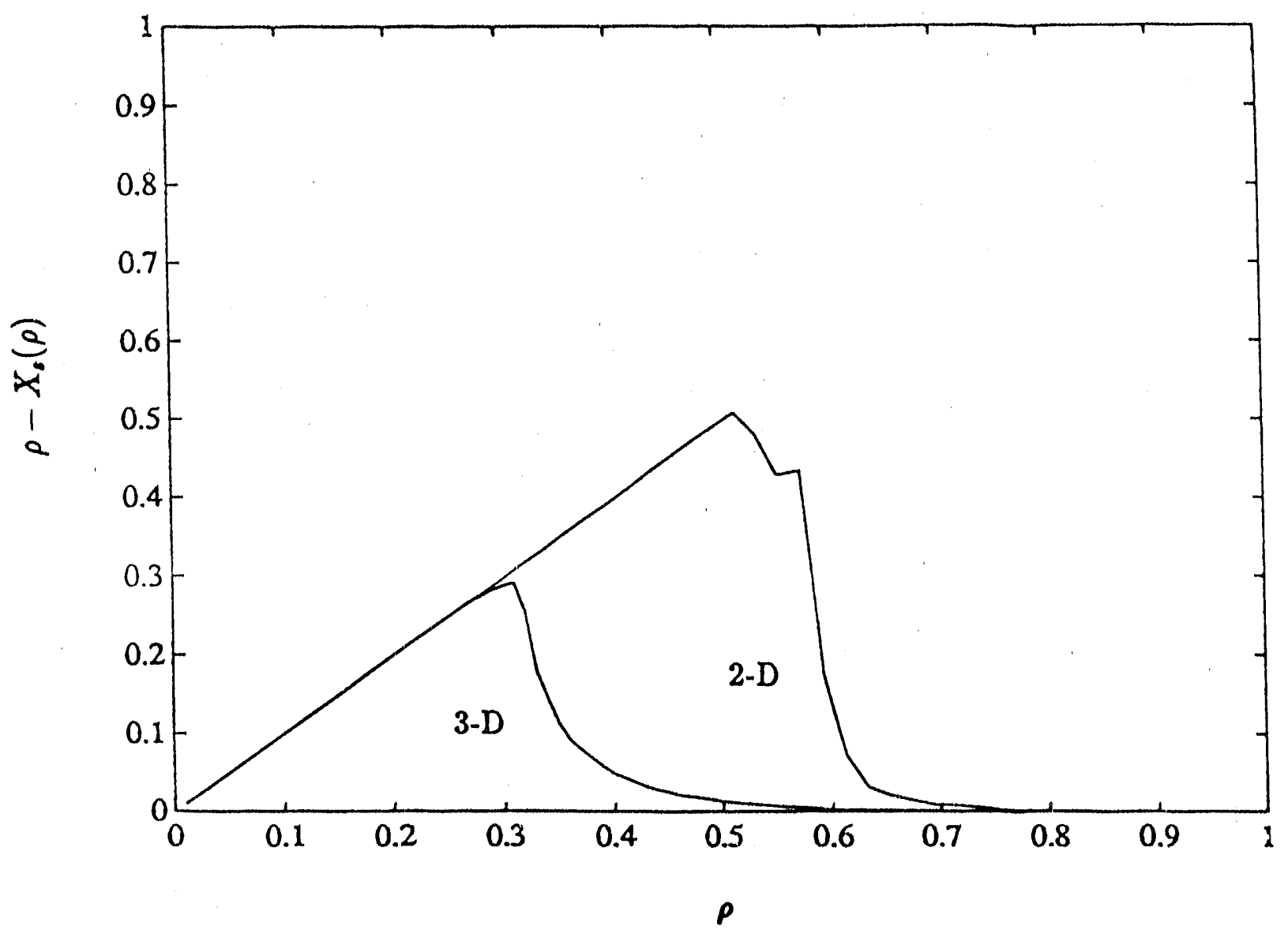

Figure 62: Trapping functions for 2-D and 3-D site percolation. 
To calculate individual phase (relative) permeabilities, the conductances of each region, determined by the phase content of each element, must be additionally known. To proceed, we assume that the dimensionless relative conductances are only functions of the individual saturation of each element and that they do not vary with the absolute permeability itself. Again, more elaborate dependences are possible, for example when the functional form $k_{r i}$ itself is being distribul 3 d, at the expense of lengthier calculations. In this work, each region would be assigned individual phase conductances according to

$$
g_{i}=k k_{r i}\left(S_{w}\right) \quad ; i=w, n w
$$

Here, $k$ is distributed with its $p d f, f(k)$, while $S_{w}$ has the more conplicated distribution described in the previous sections.

In principle, the algorithmic calculation is straightforward: At a given value of $k_{d}$, the distribution of the flow conductivity of each phase $(w, n w)$ is calculated with the use of (122). The actual details can be quite complex, since the correspondence between $k$ and $k_{r i}\left(S_{w}\right)$ is not necessarily monotonic. Knowledge of the conductivity distribution allows for an effective, large scale conductivity to be evaluated, either by direct simulation or by use of EMA or percolation theories. As is apparent in the above, in all but the simplest case, direct numerical simulation should be the prefered alternative. Upon subsequent normalization with the large scale single-phase permeability, a large scale phase permeability can be constructed for each large scale saturation $\left\langle S_{w}\right\rangle$.

A numerical example for a 2-D square lattice based on the distribution and the algorithm of Figure 57 and the model relative permeability functions $k_{r w}=S_{w}^{3}, \quad k_{r n w}=\left(1-S_{w}\right)^{2}$ is shown in Figure 63. The difference between local and large scale values should be noted. In particular, significant changes appear mostly for the non-wetting phase, the permeability of which is notably higher. Almost unaffected remains the permeability of the wetting phase. The contrast is exaggerated in the semi-log plot of Figure $63 \mathrm{~b}$. This behavior has been consistently observed for a variety of other distributions (e.g. Figure 64) and it is an important manifestation of capillarity heterogeneity. The non-wetting fluid occupies mostly high permeability regions, which are more readily drained while they also have higher flow conductivity. As a result, the permeability to the non-wetting phase rises faster than in each local element. The opposite is true for the wetting fluid. It follows that the permeability (capillary) heterogeneity, under conditions of local capillary 
control, would render a system more strongly wet in a macroscopic sense.

For a concrete, but simplified, example we shall take again the bimodal distribution of the last section, assume straight-line local relative permeabilities for convenience and use $E M A$ on a cubic lattice. We recall that given a conductivity distribution function $G(g)$ in a cubic lattice, the $E M A$ value $g_{m}$ is (Kirkpatrick [44])

$$
\int_{0}^{\infty} \frac{G(g)\left[g-g_{m}\right] d g}{g+2 g_{m}}=0
$$

For the particular case of the bimodal distribution (114) the above reduces to a quadratic that can be explicity solved. For instance, the large scale average permeability $k_{m}$ is the solution of

$$
k_{m}^{2}-\frac{1}{2} k_{m}\left[3 k_{1} \rho-k_{1}+2 k_{2}-3 k_{2} \rho\right]-\frac{k_{1} k_{2}}{2}=0
$$

To construct the relative phase conductivities it sufficppes to find their $p d f$. We follow the previous analysis:

At stage $1\left(k_{1}<\frac{k_{2}}{\alpha}<k_{d} \leq k_{2}\right)$, we have a set of volume fraction $\rho$ with absolute permeability $k_{1}$, and $S_{w}=1$, hence $g_{w}=k_{1}, g_{r \cdot w}=0$; and a set of volume fraction $1-\rho$ with absolute permeability $k_{2}$, and $S_{w}=I\left(\frac{k_{2}}{k_{d}}\right)$, hence $g_{w}=k_{2}\left[\frac{\left\langle S_{w}\right\rangle-\rho}{1-\rho}\right] g_{n w}=k_{2}\left[\frac{1-\left\langle S_{w}\right\rangle}{1-\rho}\right]$, where use was also made of (115). The conductivity distribution of either phase is bimodal

$$
G_{i}(g)=\rho \delta\left(g-g_{i 1}\right)+(1-\rho) \delta\left(g-g_{i 2}\right) \quad ; \quad i=w, n w
$$

with

$$
g_{w 1}=k_{1}, \quad g_{w 2}=k_{2}\left[\frac{\left\langle S_{w}\right\rangle-\rho}{1-\rho}\right] ; \quad g_{n w 1}=0, \quad g_{n w 2}=k_{2}\left[\frac{1-\left\langle S_{w}\right\rangle}{1-\rho}\right],
$$

thus (123) can be applied accordingly. In particular, the large scale relative conductance for the non wetting phase is linear in this region, just like the local curve, although with a higher absolute slope, $k_{2}(2-3 \rho) / 2 k_{m}(1-\rho)$ (Figure 65). The wetcing phase permeability, on the other hand, is generally non-linear and smaller than the local curve, as also anticipated. These results are consistent with the observations made in the numerical simulations of the full problem (Figures 63, 64). The large scale relative permeabilities attribute to the system features of stronger wettability.

At stage 2 there is change only for $\frac{k_{1}}{\alpha} \leq k_{d}<k_{1}$. Now, we have three sets: set $(a)$ of fraction $X_{\bullet}(\rho)$ with permeability $k_{1} S_{w}=I\left(\frac{k_{1}}{k_{d}}\right)$, hence $g_{w}=\frac{k_{1}}{X_{\bullet}(\rho)}\left[\left\langle S_{w}\right\rangle-\rho+X_{d}(\rho)\right]$ and $g_{n w}=$ 


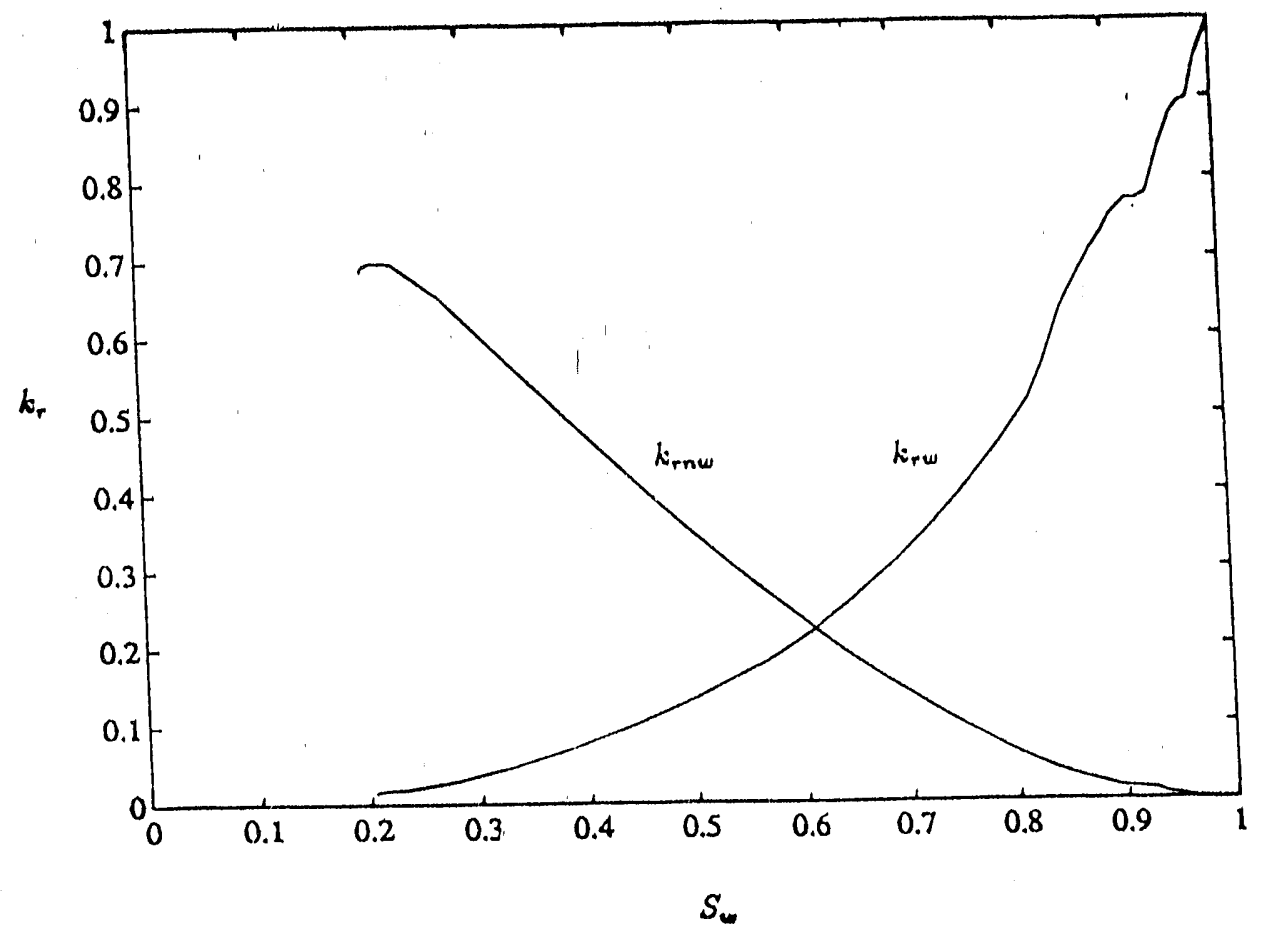

(a)

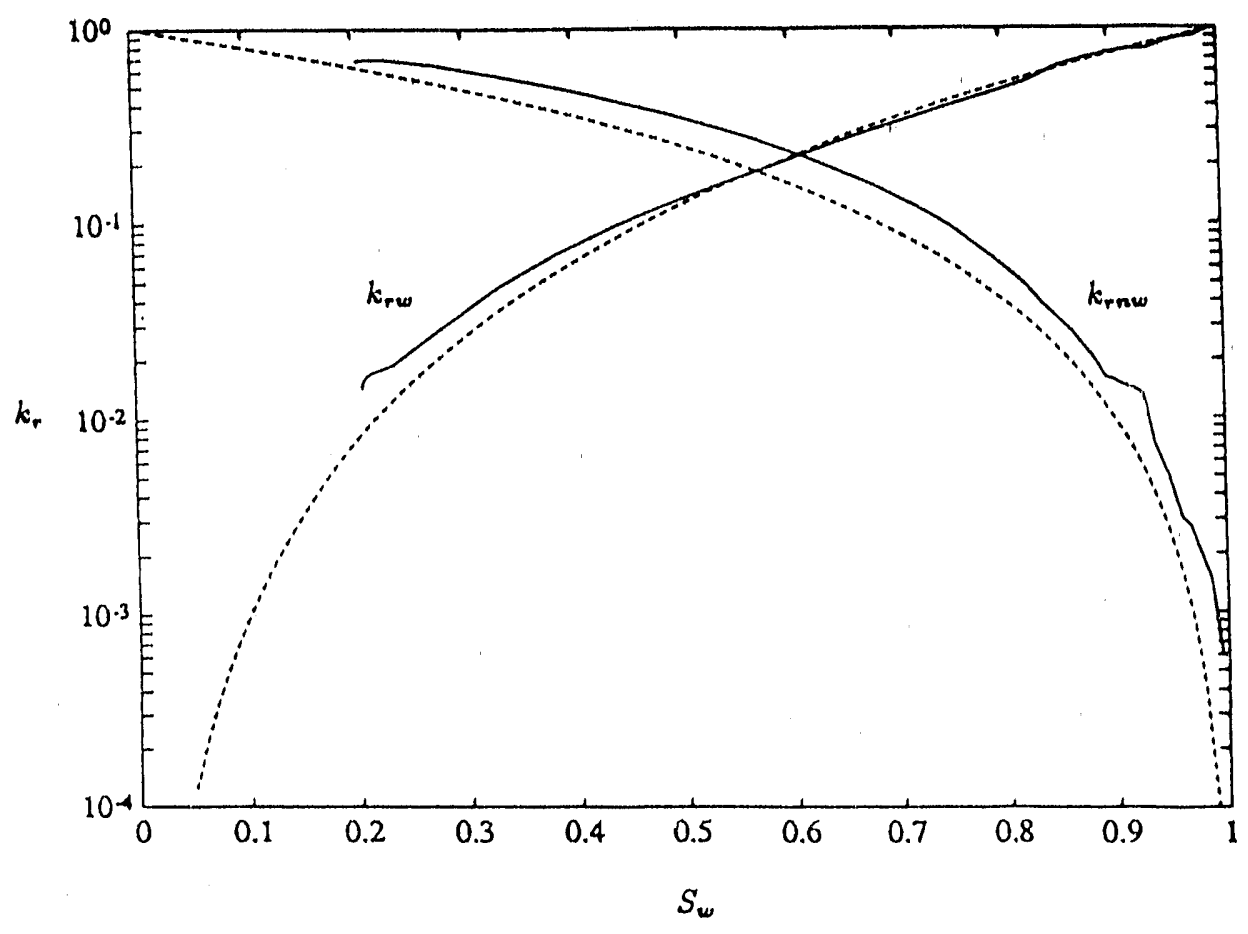

(b)

Figure 63: Large scale relative permeabilities. 


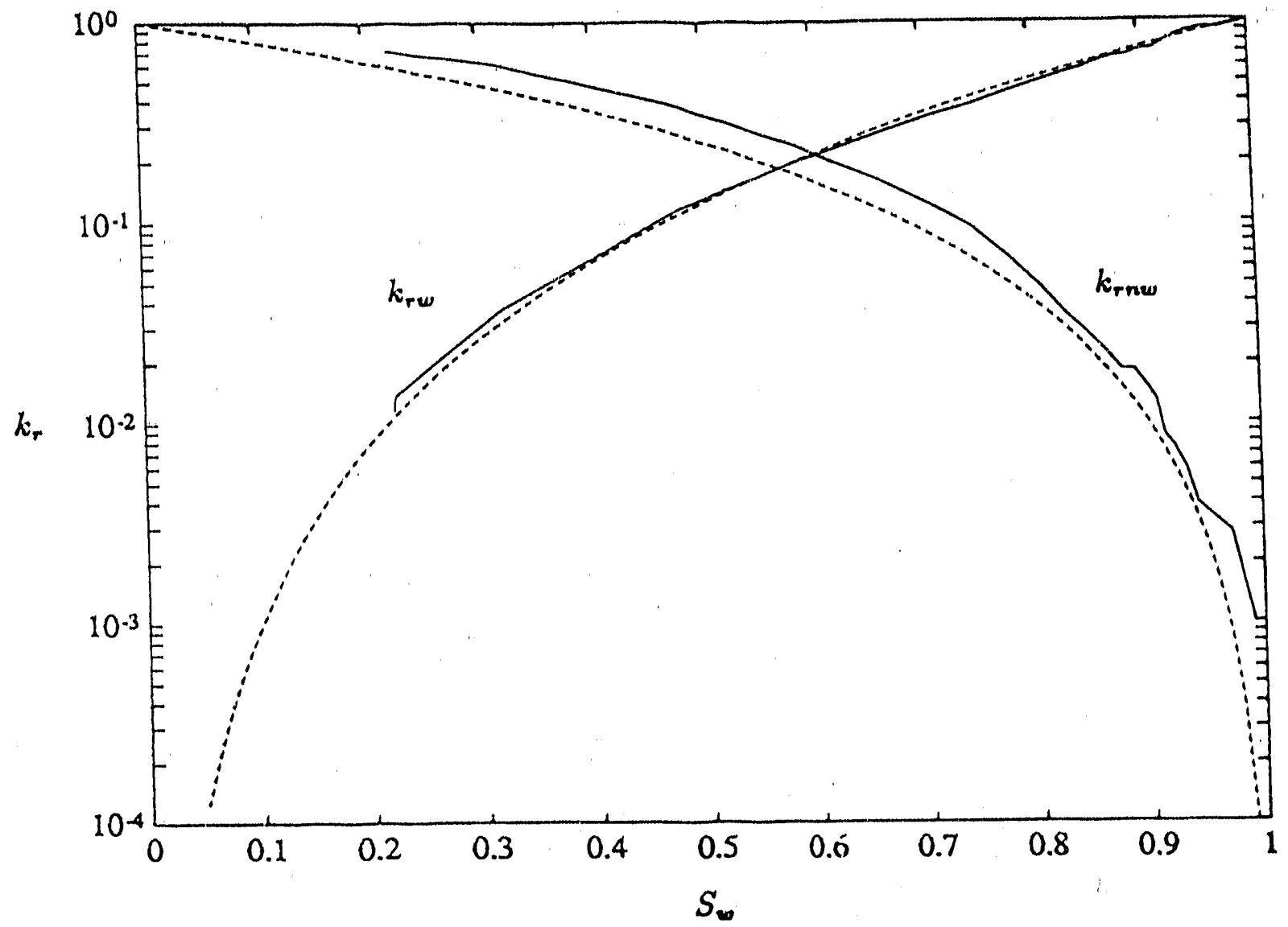

Figure 64: Semi-log plot of large scale averages.

$\frac{k_{1}}{X_{0}(\rho)}\left[\rho-\left\langle S_{w}\right\rangle\right]$, where use was also made of $(119)$; set $(b)$ of fraction 1 - $\rho$ with permeability $k_{2}$, $S_{w}=0$, hence $g_{w}=0$, and $g_{n w}=k_{2}$; and set $(n a)$ of fraction $\rho-X_{s}(\rho)$ with $S_{w}=1$, hence $g_{w}=k_{1}$, and $g_{n w}=0$. Distributions are again a collection of delta functions which allows for explicit computations. In particular, since zero conductance elements exist for both phases, the resulting equations are quadratic. The computed results are shown in Figure 65. For the specific example taken, the wetting phase permeability has decreased rapidly and remains small throughout the remainder of the process. We should emphasize that some of these features reflect the restrictive approximations of bimodal distribution and the use of EMA. Nevertheless, the non-trivial aspects of the averaging process should be apparent.

\subsection{CONCLUDING REMARKS}

In this paper, a statistical approach was proposed for the large scale averaging of primary drainage under conditions of local capillary equilibrium. The approach employs extension of percolation theory in its various forms (ordinary, invasion, and percolation with trapping) to yield large scale capillary pressure and relative permeability functions. Several restrictions are nessary for the method to be applicable, the most severe of which are the absence of long-range correlations in 


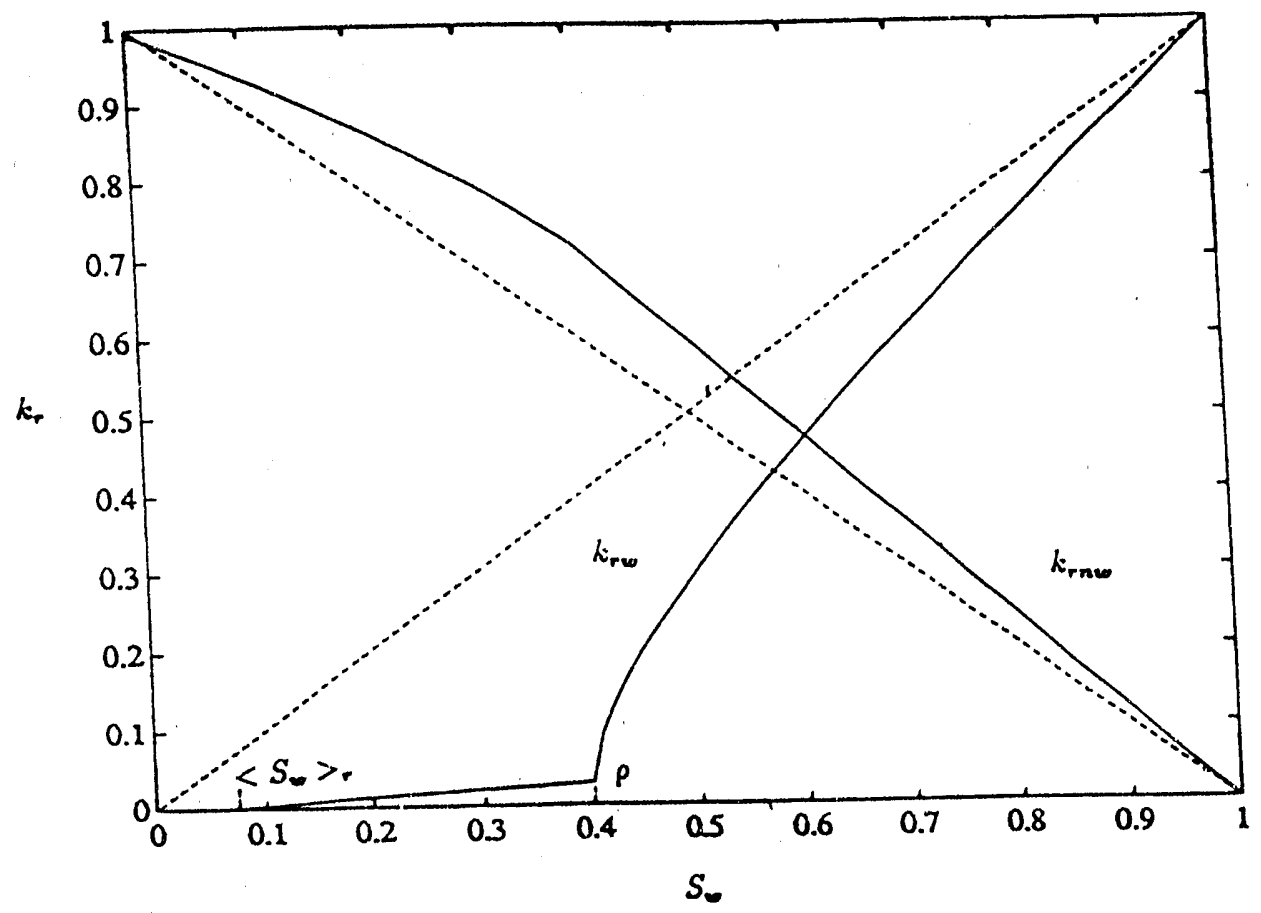

(a)

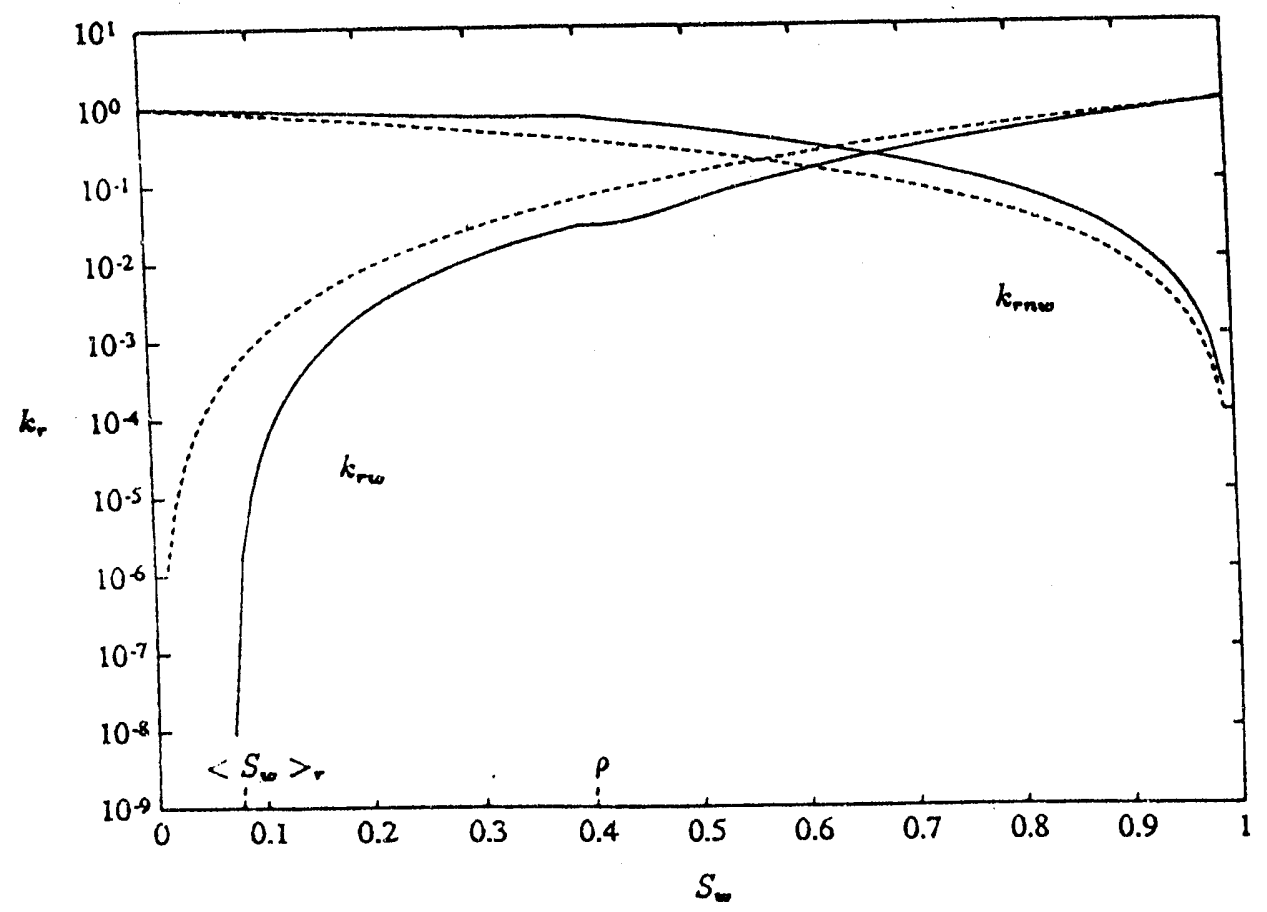

(b)

Figure 65: Large scale relative permeabilities for Figure 61 using EMA. 
method to be applicable, the most severe of which are the absence of long-range correlations in permeability (in fact a completely disordered field was assumed) and the existence of local capillary equilibrium. Because of these limitations, the averaging is expected to be more relevant for scales generally smaller than the field scale. Scale limitations of percolation patterns were quantitatively addressed by Jenormand [50] in his classification of displacement processes. Analogous constraints can also be developed for the present problem, although they are not attempted here. Certainly, capillary dominated displacement would be increasingly difficult to hold as the length scale of heterogeneity increases. Non-local models, largely undeveloped at present, are necessary under such conditions.

However, in case of capillary control, the method presented in this paper is the fundamentally proper approach for the derivation of large scale average multi-phase functions, such as capillary pressure and relative flow conductivities. As is evidenced in this paper, the latter should depend on the direction of the displacement (whether primary or secondary, drainage or imbibition) and the degree of heteregeneity, and they should exhibit large scale percolation features, such as invasion and trapping thresholds. For primary drainage, a large scale trapped saturation was identified, the existence of which is due to large scale trapping, although its specific value depends significantly on the individual capillary characteristics. One suspects that large scale trapping would likewise lead to an augmented residual non-wetting saturation in imbibition processes. This can be of significance to oil recovery processes. Progress in that direction, however, must await the satisfactory resolution of imbibition processes at the pore scale Bacri et al. [5]. Finally, simulations of large scale relative permeabilities showed that the large scale capillary heterogeneity attributes to the system features of wettability stronger than the actual. Although novel, this behavior is, nevertheless, expected. We close by pointing our that the proposed extension of percolation with trapping under conditions of partial occupancy could be also directly applicable to pore-level modeling when a pore is only partially cooupied by a phase (for instance, under conditions of intermediate wettability). 


\title{
7 LONG WAVES IN PARALLEL FLOW IN HELE-SHAW CELLS
}

\author{
M. Zeybek and Y.C. Yortsos
}

\subsection{INTRODUCTION}

The flow of two immiscible fluids in a Hele-Shaw cell has been a subject of great interest. The majority of studies have focused on frontal displacement, particularly on viscous fingering (VF) $[9,38,63,74,94]$. This is readily understood in view of the interesting problems in the high $N_{c a}$ limit [19], the relation of VF to Laplacian growth [64] as well as in view of consequences to oil recovery [28]. As a result, little or no attention has been paid to interfaces parallel to the main flow direction. While such configurations lack the richness of frontal motion, their study is nonetheless desirable. Parallel flow is the theoretical limit of fully developed fingers (e.g. the Saffman-Taylor finger). Even in the absence of capillarity, this regime is of great interest, especially for finite viscosity ratio $[43,49]$. In porous media, parallel flow is often encountered in thin reservoirs, usually masked under the assumption of vertical flow equilibrium (VFE) $[18,27,108]$. Finally, interfaces parallel to the main flow may support motion similar to shallow water waves, the Laplace equation being the field equation in both cases. The latter possibility has obvious interests of its own and forms the subject of this chapter.

\subsection{THEORY}

We study the interfaces between two immiscible fluids of different viscosities in the parallel HeleShaw flow schematic of Figure 66. The cell is horizontal and has thickness W. Parallel flow requires flat interfaces and the absence of transverse pressure gradients. Under the usual assumptions the latter requires $\mu_{b} q_{b}=\mu_{a} q_{a} \equiv Q$, where subscript $a$ indicates the "inner" fluid, so that a viscosity ratio can be defined as usual, $M=\mu_{b} / \mu_{a}$. We denote by $\lambda_{1}$ and $\lambda_{2}$ the normalized interface positions $\left(-1 \leq \lambda_{2} \leq \lambda_{1} \leq 1\right)$. Of particular interest is the symmetric case $\left(\lambda_{1}=-\lambda_{2}\right)$, the Saffman-Taylor finger corresponding to $\lambda_{1}=-\lambda_{2}=1 / 2, M=\infty$.

For preliminary results, we consider a linear analysis in the absence of capillarity, using normal modes $\left(e^{\omega t+i \alpha x}\right)$ for disturbance amplitudes. For the symmetric case, we obtain the compact 


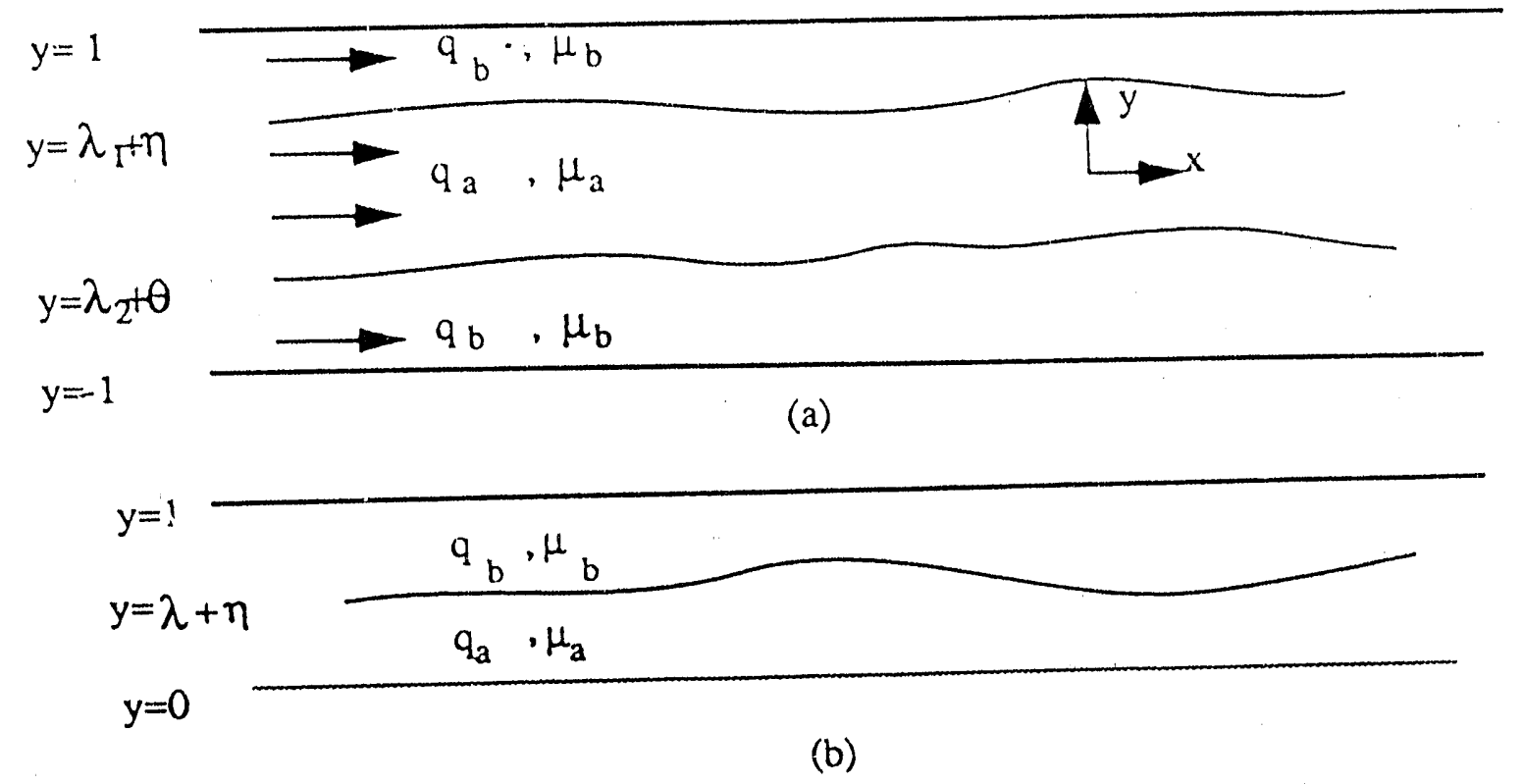

Figure 66: Flow geometry for (a) non-symmetric and (b) symmetric cases.

dispersion relation

$$
\omega=-(2 i Q \alpha \sinh \alpha) /\left(\left(\mu_{a}+\mu_{b}\right) \sinh \alpha+\left(\mu_{a}-\mu_{b}\right) \sinh \alpha\left(1-2 \lambda_{1}\right)\right)
$$

For the non-symmetric case $\left(\lambda_{1} \neq-\lambda_{2}\right)$, asymptotic expansions at large wavelengths are convenient. Two solutions exist

$$
\omega_{k}=i Q \alpha\left(x_{0, k}+x_{2, k} \alpha^{2}+\ldots\right) \quad ; k=1,2
$$

where $x_{0,1}=-1 / M, \quad x_{0,2}=-2 / \Delta, \quad x_{2,1}=(M-1)\left(\lambda_{1}-1\right)\left(\lambda_{2}+1\right)\left(\lambda_{1}-\lambda_{2}\right) /\left(M^{2}\left(2+\lambda_{2}-\lambda_{1}\right)\right)$, $x_{2,2}=4(M-1)\left(\lambda_{1}-\lambda_{2}\right)\left(1+\lambda_{2}-\lambda_{1}\right)\left[\left(\lambda_{2}+1\right)^{2}+\left(\lambda_{1}-1\right)^{2}+\left(\lambda_{2}+1\right)\left(\lambda_{1}-1\right)\right] / 3 \Delta^{2}\left(2+\lambda_{2}-\lambda_{1}\right)$, and where $\Delta \equiv 2+(M-1)\left(\lambda_{1}-\lambda_{2}\right)$. In the above, we neglected capillarity (which enters at $O\left(\alpha^{3}\right)$ ) assuming large $N_{c a}=q_{a} \mu_{a} / \gamma$ (but not too large for 3-D effects to be important [53, 59]). Equivalently, the previous pertain to long waves. Equations (127)-(128) predict dispersive waves $[1,25]$ for $M \neq 1$ (imaginary $\omega$ and $\alpha$-dependent wave velocity). The waves are non-dispersive for $M=1$.

Subsequently, the non-linear evolution of small amplitude long waves was considered following an asymptotic approach $[42,160]$. We assume incompressible fluids, the pressure of which satisfy 
$\nabla^{2} P=0$, and continuity of pressure and mass across the interfaces. In the following, time is scaled by $\mathrm{L} / q_{a}$, interface amplitudes by $\mathrm{W}$, and streamwise lengths by $\mathrm{L}$, a measure of the initial disturbance. The asymptotic analysis is one of small amplitude $(\epsilon)$, long waves $\left(\epsilon=O\left(\delta^{2}\right), \delta=\right.$ $\mathrm{W} / \mathrm{L}, \epsilon, \delta \ll 1)$ and long time $(\bar{t}=\epsilon t)$ and pertains to the initial value problem. Here, $\epsilon$ denotes the initial normalized amplitude and $\delta \equiv W / L$. Details will be published elsewhere. The final result is a set of $\mathrm{KdV}$ and Airy equations

$$
\begin{gathered}
V_{\tilde{t}}-\frac{(M-1)}{M \Delta} a_{1} V_{\xi \xi \xi}=0 \\
U_{\tilde{t}}-\frac{4(M-1)}{\Delta^{2}}\left(2+\lambda_{2}-\lambda_{1}\right) U U_{\sigma}-\frac{(M-1) \kappa^{2}}{M \Delta} a_{2} U_{\sigma \sigma \sigma}=0
\end{gathered}
$$

where $V$ and $U$ are linearly related to the two interface amplitudes $\eta$ and $\theta$ (Figure 66a) :V= $-\left(\left(1+\lambda_{2}\right) \eta+\left(1-\lambda_{1}\right) \theta\right) /\left(2-\lambda_{1}+\lambda_{2}\right), \quad U=(\eta-\theta) /\left(2-\lambda_{1}+\lambda_{2}\right)$. The equations are in two different moving coordinates $\xi$ and $\sigma, \xi=x-t / M, \sigma=x-2 t / \Delta$. The parameters in (129)-(130) depend on $\lambda_{1}, \lambda_{2}$, and $M: a_{k}=M \Delta x_{2, k} /(M-1), \delta=\kappa \sqrt{\epsilon}$.

We note the following : (i) Translational motion occurs for $M=1$; (ii) The advective speed $(1 / M, 2 / \Delta)$ decreases to zero at large $M$; (iii) The dispersive term in the KdV equation vanishes for $1+\lambda_{2}-\lambda_{1}=0$ (which in the symmetric case, coincides with the Saffman-Taylor condition $\left.\lambda_{1}=1 / 2\right) ;(i v)$ Anti-symmetric disturbances $(\eta=\theta)$ are governed by the linear Airy equation alone; $(v)$ The symmetric problem $\left(\lambda_{1}=-\lambda_{2}\right.$ and $\left.\eta=-\theta\right)$ is governed by the KdV equation alone. The latter is the case for the subsequent experiments (Figure $66 \mathrm{~b}$ ), where we can rewritte

$$
\eta_{t}+\frac{2}{\Delta} \eta_{x}-\frac{8(M-1)}{\Delta^{2}} \eta \eta_{x}+\epsilon \frac{4}{3} \frac{\kappa^{2}}{\Delta^{2}}(M-1) \lambda(1-2 \lambda)(1-\lambda) \eta_{x x x}=0
$$

Although we also use the exact results of Inverse Scattering $[1,25]$, we shall rely mostly on the numerical pseudospectral technique of Fornberg and Whitham [29] for subsequent comparison with experimental results. We might add that finite amplitude equations describing breaking waves were also derived but will not be discussed here.

\subsection{EXPERIMENTS}

To test the theory, a long and narrow Hele-Shaw cell was constructed consisting of two plexiglass plates 0.5 in. thick, of dimensions $220 \mathrm{~cm} \times 27 \mathrm{~cm}$, separated by a rubber spacer $0.08 \mathrm{~cm}$ thick and $10 \mathrm{~cm}$ wide. The set up consists of the cell, two peristaltic pumps and video equipment. 
Although other fluid pairs were also used, the experiments below were conducted with mineral oil and glycerol/water solution, with viscosities $170 \mathrm{cp}$ and $860 \mathrm{cp}$, respectively, and with capillary typically number of $O(0.01)$. No sustained propagation was observed for substantially lower $N_{c a}$. The experiments involved only one interface (Figure $66 \mathrm{~b}$ ), thus pertaining to the symmetric case only. The solution of (129)-(130) is invariant to the change $\lambda_{1} \rightarrow 1+\lambda_{2}, \eta \rightarrow \theta, M \rightarrow 1 / M$ (note also the rescaling of time), thus we may view the flow as the top half of a symmetric problem, the "lower" fluid being fluid $a$. In our experiments, this was the more viscous fluid (thus, $M=0.2$ ). Because of the particular configuration, only the symmetric part of the theory was tested.

Conducting the flow experiments consists of $(i)$ establishing a flat fluid interface and parallel flow conditions $\left(\mu_{b} q_{b}=\mu_{a} q_{a}\right)$; and (ii) monitoring the motion of disturbance initiated by interrupting momentarily the flow of one fluid. Existence of solitons can be tested by comparison with simulation, but more conveniently by looking for key properties [25]: (i) Arbitrary disturbances evolve into one or more solitons and dispersive waves; (ii) The speed of a soliton increases with an increase in amplitude; and (iii) Solitons regain their identity after interaction. Typical results from still pictures taken from a videotape are shown below. According to the theory, the following is expected: forward moving (with respect to $\sigma$ ) solitons for $\lambda>1 / 2$ and positive initial disturbance; backward moving (with respect to $\sigma$ ) solitons for $\lambda<1 / 2$ and negative initial disturbance; and dispersive waves oth swise. It must be pointed out that once the disturbance exits the cell, the original interface is recovered, thus the experiments can be easily reproduced.

Figure 67 a shows typical initial disturbance imposed on the parallel interface. Upon restoration of the flow, the disturbance is advected, while also starting to develop into a wave of constant shape followed by a wiggly interface of small amplitude and short wavelength. Typically, this wave takes a permanent form after traveling about $45 \mathrm{~cm}$ (Figure 67b), and appears to possess all the characteristics of a soliton. Its amplitude is different from the initial and remains constant for a substantial distance traveled (Figure 67c) (as long as $150 \mathrm{~cm}$, beyond which end effects become appreciable). A simulation based on the initial shape of Figure 67a is shown in Figure 68. Good agreement between theory and experiment is obtained, despite the ambiguity on the suitability of the initial condition in the simulation (due to the flow interruption). We note that features typically associated with soliton and dispersive waves are suppressed in the scales of Figure 68 , that match the experiment. We illustrate this point by replotting the profile in the inset with 

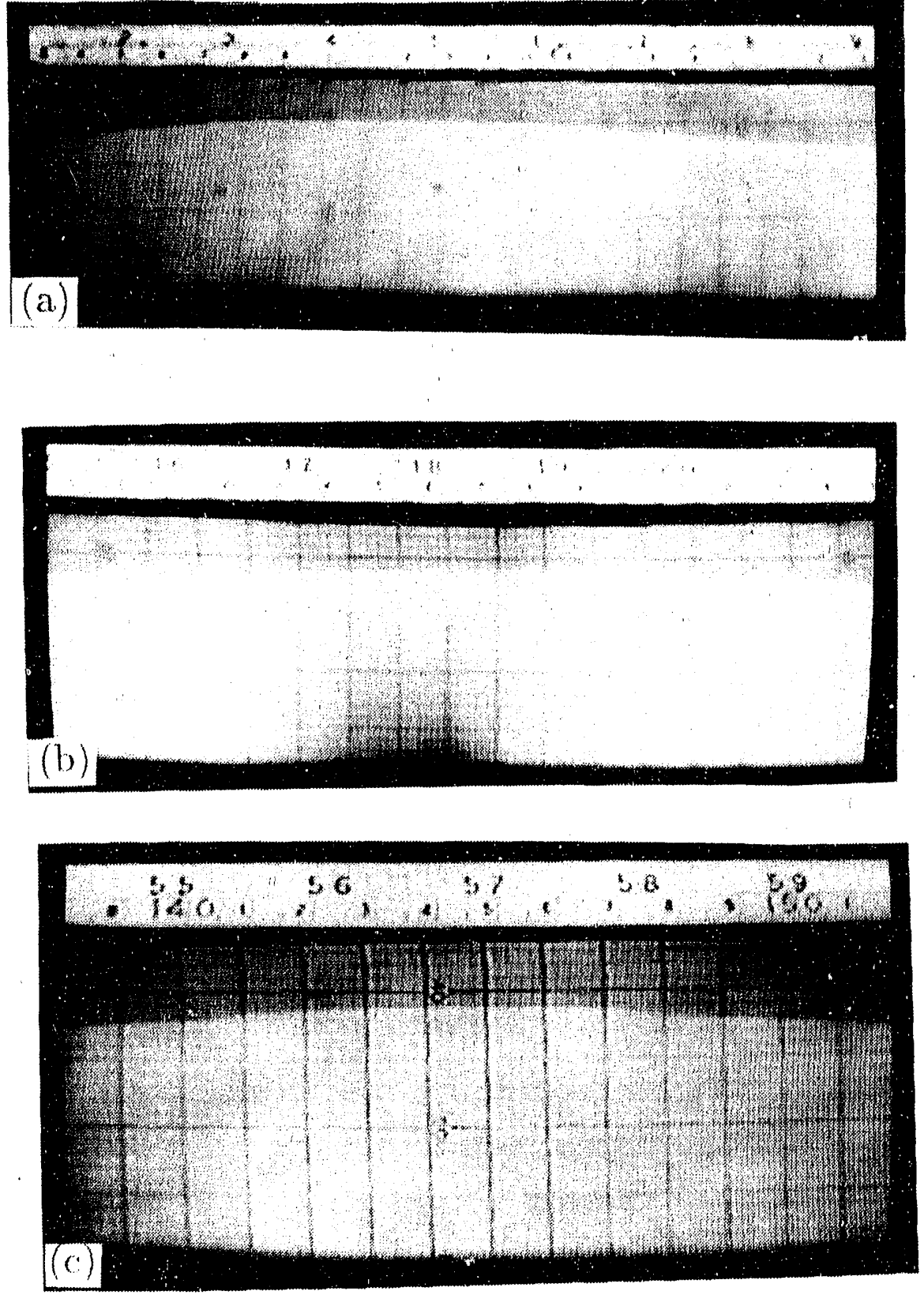

Figure 67: Single solitary wave. (a) Initial condition, (b) and (c) subsequent stages. 
different scales. We must add that motion of dispersive waves was not observed to our satisfaction in the experiments. A noisy interface of small wave length and amplitude did indeed form soon after the main wave evolved. However, we suspect that wettability and surface tension played a major role on its subsequent development.

Figure 69 shows the formation of two solitons arising from a longer initial disturbance. Two solitary waves of different amplitudes are expected. As tine progresses, the two solitons separate (Figure 69b), the higher amplitude moving faster and away from that of lower amplitude. Comparison with the numerical simulations is again quite satisfactory (Figure 70, see also inset with different scaues). Soliton interaction is shown in Figure 71 . The two waves were created by sequentially introducing two disturbances of different amplitude. The second is of higher amplitude and evolves into a faster soliton that eventually overtakes the preceding one. After interaction, tall and short solitons reappear, but in reverse order, and they propagate with their original speed (Figure 71b). Corresponding numerical simulations are shown in Figure 72.

As previously mentioned, a variety of other conditions were also investigated and found consistent with the theory. Short wave disturbances dissipate shortly after their onset. For solitons that propagate in the negative $\sigma$ direction $(\lambda<1 / 2)$, dispersive waves should proceed them in a fixed frame of reference. Such were noticed, although the above remarks on wettability effects apply here as well. For $M=1$, constant wave speed and motion independent of the amplitude are expected. These features were indeed observed for nearly equal viscosity fluids. Furthermore, for $M \sim 1$, large amplitude waves did not break, in contrast to every other case studied.

In summary, we presented in this chapter, an analysis of the wave motion of the interface of immiscible fluids in viscous-dominated parallel flow in a long Hele-Shaw cell. Small amplitude, long wavelength disturbances were shown to be governed by KdV and Airy equations. For the symmetric problem, experimental evidence was supportive of the theory, including the propagation of solitary waves. 


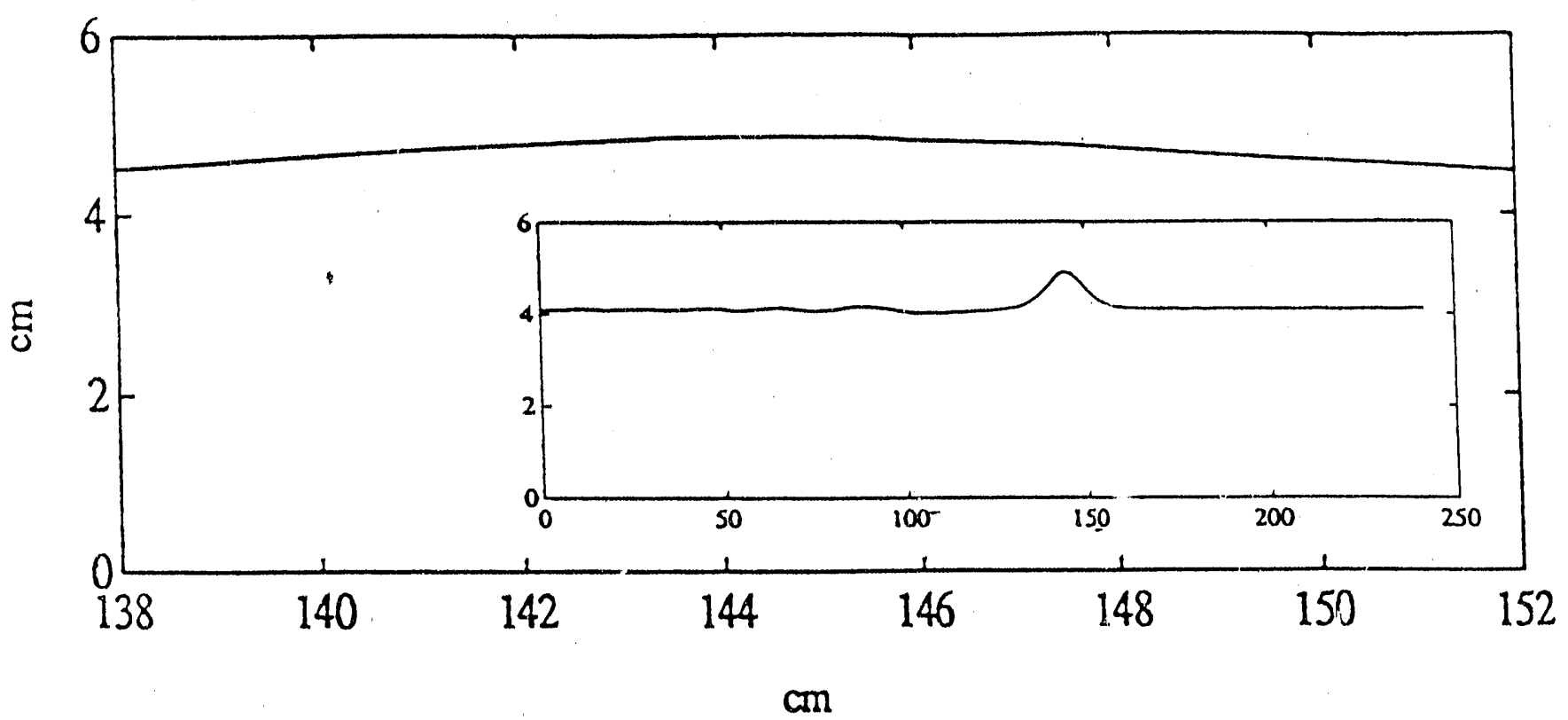

Figure 68: Numerical simulation of Figure 67. Inset: profile in different scales. 

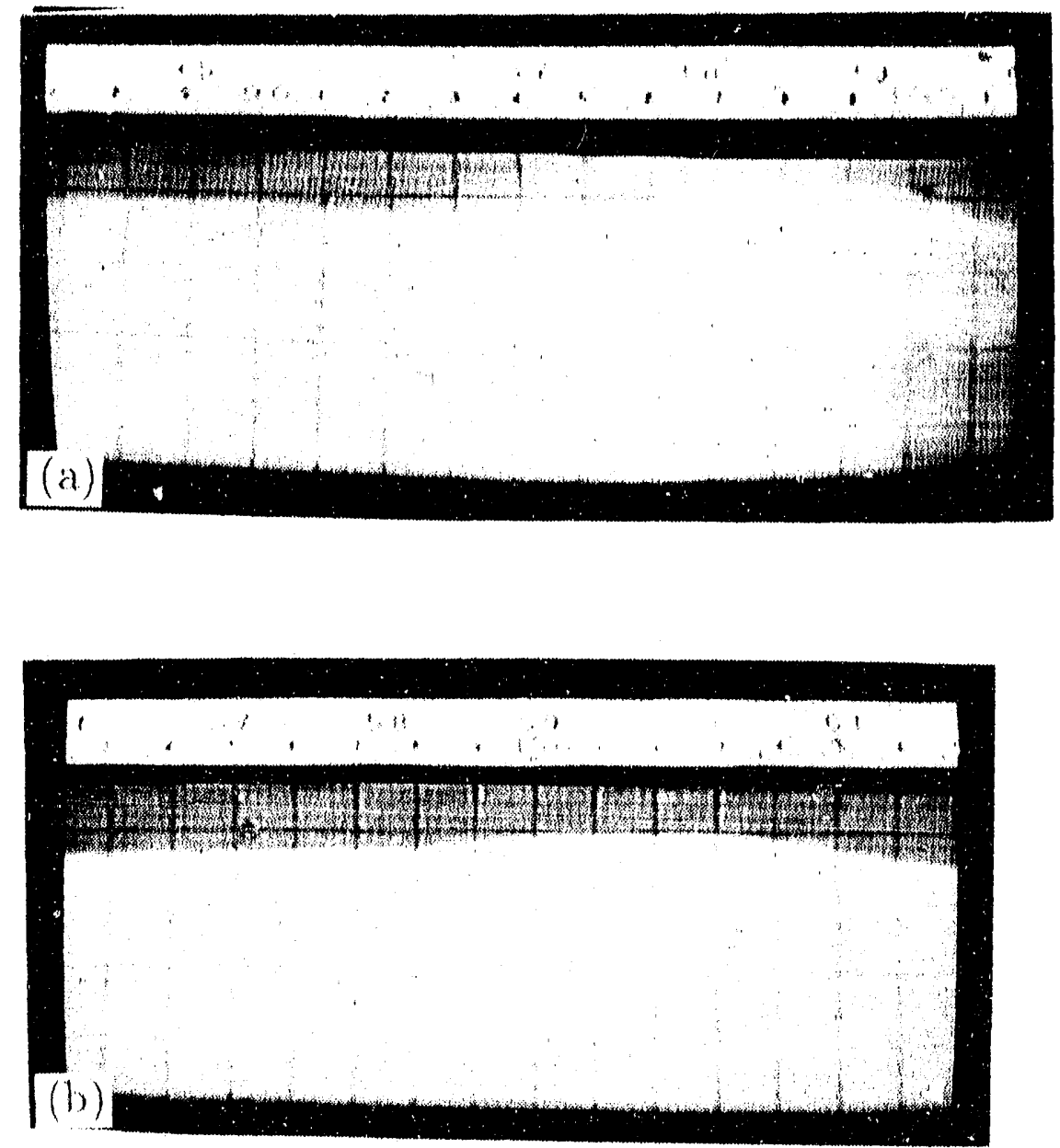

Figure 69: Two solitary waves at two different stages. 

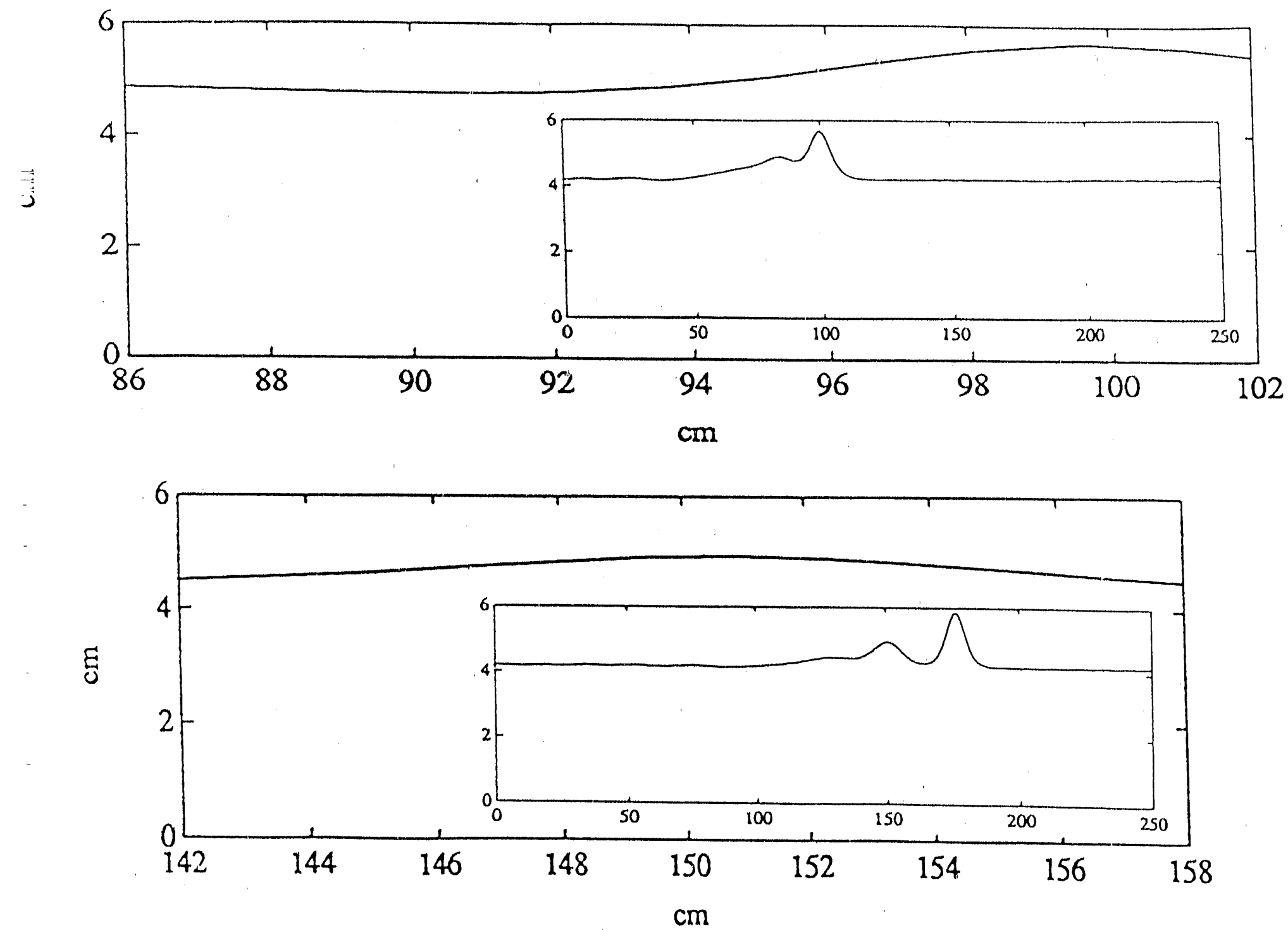

Figure 70: Numerical simulation of Figure 69. Inset: profile in different scales. 

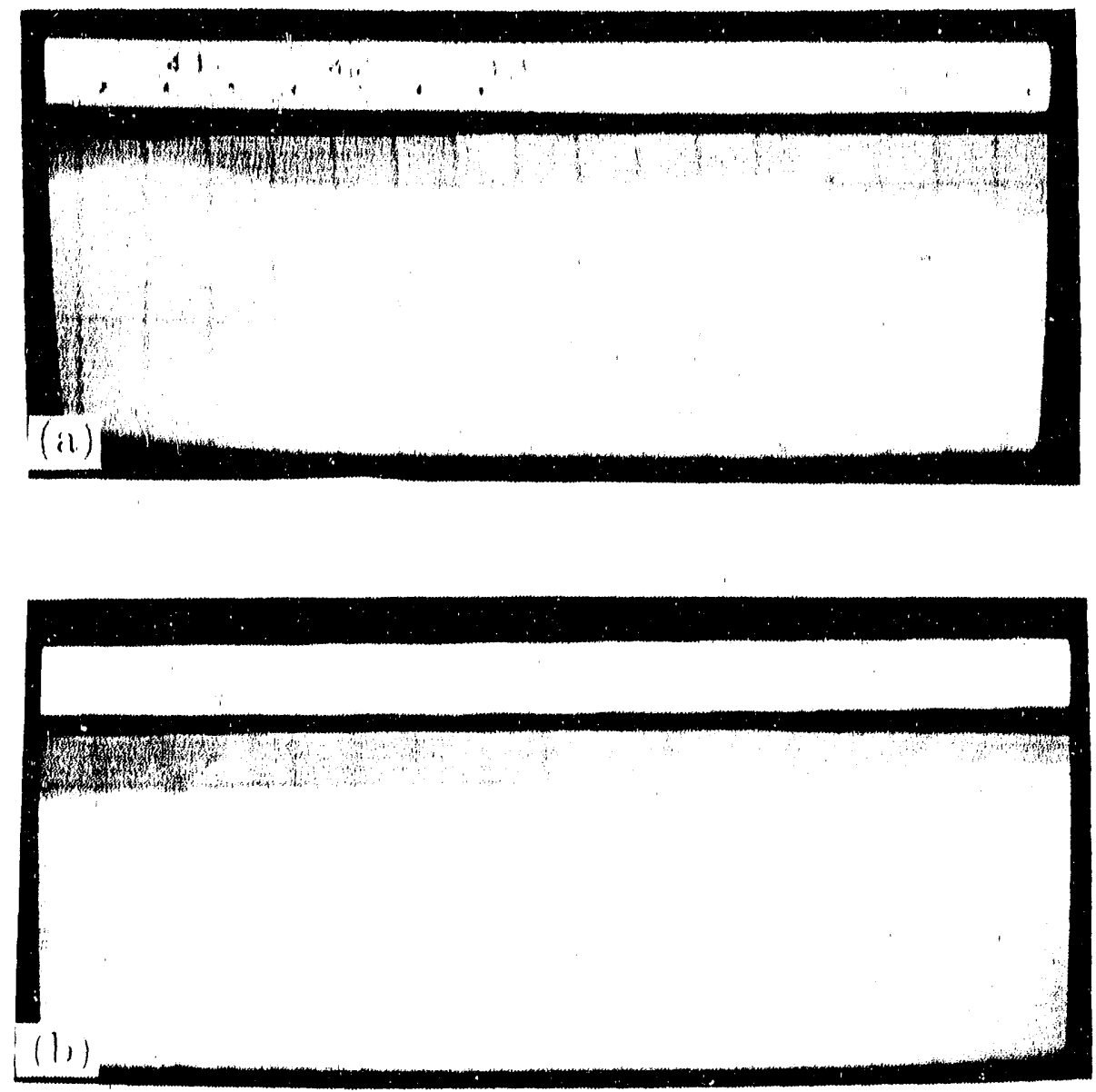

Figure 71: Two solitary waves (a) before and (b) after interaction. 

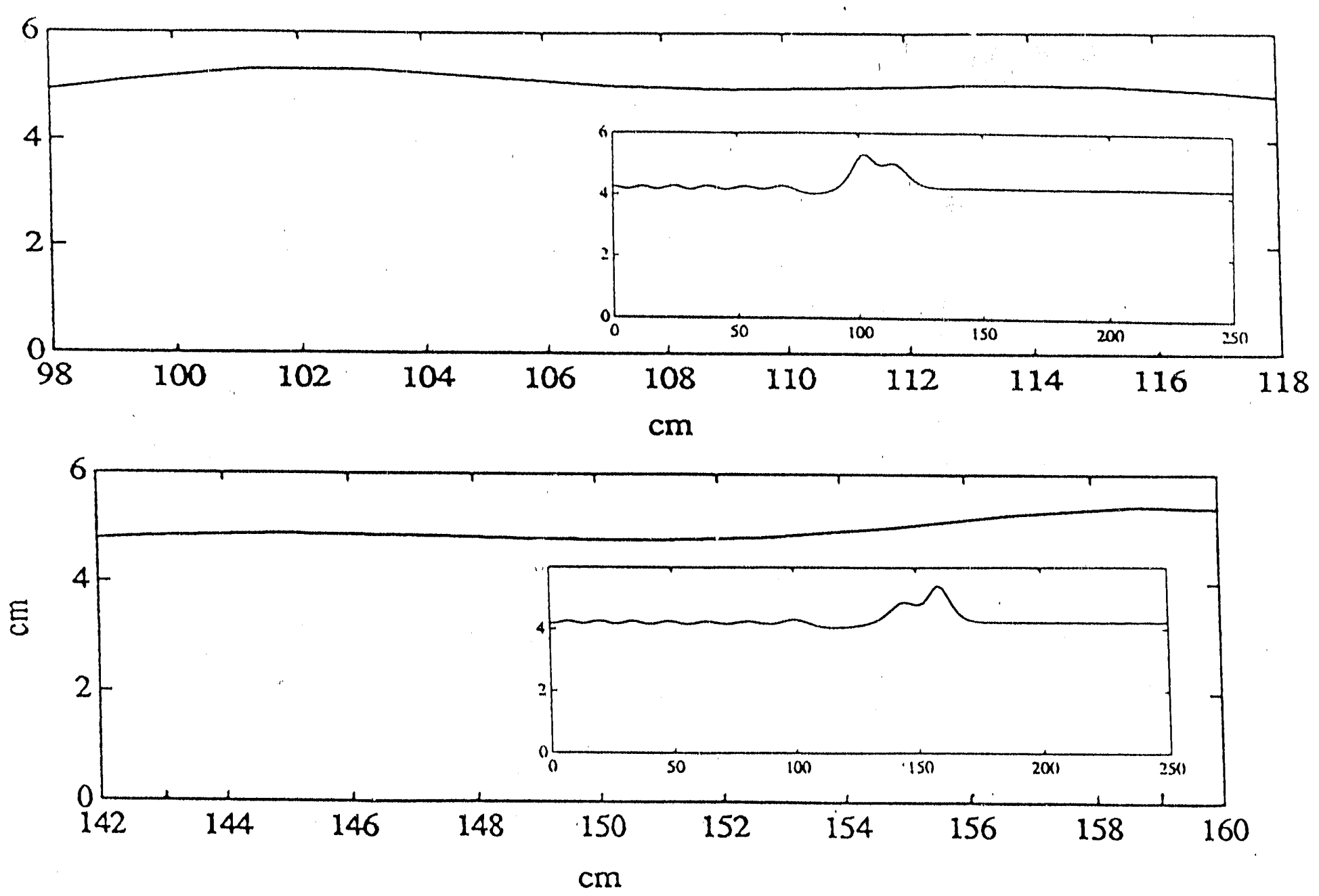

Figure 72: Numerical simulation of Figure 71. Inset: profile in different scales. 


\title{
8 MECHANISTMS OF FOAM GENERATION AND PROPAGATION IN POROUS ME. DIA
}

\author{
S.Y. Baghdikian and L.L. Handy
}

\subsection{INTRODUCTION}

Our work, in foam generation is mainly experimental and involves the simultaneous injection of surfactant solution and gas into a core pre-saturated with a surfactant solution. Of particular interest are the questions of foam generation and foam propagation into the core. Various theories for foam formation and propagation have been proposed (Chambers and Radke [13], Rossen and Gauglitz [71]), where a minimum pressure gradient or a minimum flow rate is postulated as a condition necessary for the onset of strong foam. Our research has been focusing on this aspect. We have previously identified (Annual DOE Report, 1989) an incubation period associated with the formation of foam in porous media. This time is often of the order of tens of pore volumes, although it appreciably decreases with an increase in the flow rates. Our previous experimental set up was not suitable for an in-situ monitoring of such effects. To improve our understanding, in the past year we have modified the set up and attached pressure taps at various points along the core. By so doing, we have been able to monitor the progress of foam formation and propagation. We have also conducted experiments to study the effect of injecting pregenerated foam. All these experiments are described below.

\subsection{EXPERIMENTAL RESULTS}

Experiments were conducted in a 2 in diameter, 10 in long Berea sandstone core, of porosity 0.24 and permeability 500 md. After saturating the core with brine, surfactant adsorption onto the pore surfaces was satisfied by injecting at least 10 pore volumes of surfactant solution $(0.5 \%$ Chaser SD1000 in $1 \% \mathrm{NaCl}$ brine). After adsorption was completed and while injecting the surfactant solution, gas injection also started. A check valve was added downstream of the back pressure regulator to minimize gas expansion, thereby reducing pressure fluctuations at the downstream end of the core. This significantly mirimized the downstream end pressure fluctuations observed in previously reported experiments. The experiments were initiated at high fluid velocities in order to reduce incubation time. 


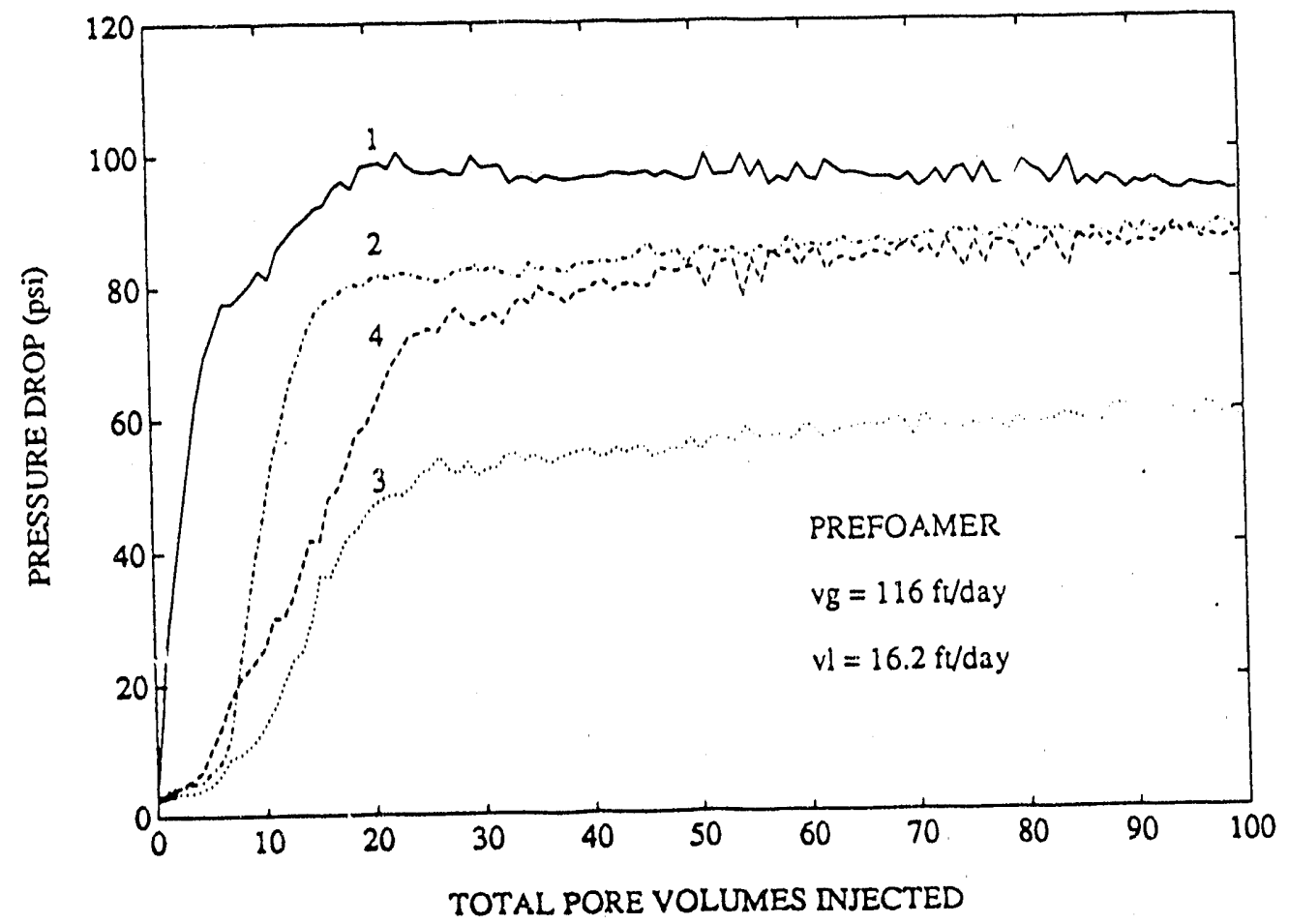

Figure 73: Pressure drop history in Berea sanstone during simultaneous surfactant and gas injection (with prefoamer).

After gas injection was initiated, the pressure in the first section of the core increased immediately (Figure 73). A delayed propagating response was observed in the other sections of the core, in the order section 2 , followed by sections 3 and 4 . The effluent section of the core had a pressure drop higher than section 3 possibly due to an end effect. About 25 PVs of fluid injection were necessary before pressure drops stabilized to fairly constant level. Nevertheless, continuous fluctuations were recorded.

Next, the effect of prefoamer was studied (Figure 74). The prefoamer used has a typical pore size of 7 microns and produces a finely textured foam. In the early time period, after gas injection was initiated the furst section responded immediately. Upon injecting about $6 \mathrm{PVs}$ of foam, the frrst section had reached a steady pressure drop. Within injection of $25 \mathrm{PVs}$, a steady pressure drop was recorded throughout the core. It appeared that foam propagated as a front through the core. Subsequently, and after steady-state foam flow was reached with the use of the prefoamer, the prefoamer bypass line was opened and the fluids were mixed in the tubing before entering the core. The first two sections of the core recorded a higher pressure drop than the last two sections, the first section being the highest, although pressure drops in all four sections were at similar levels 


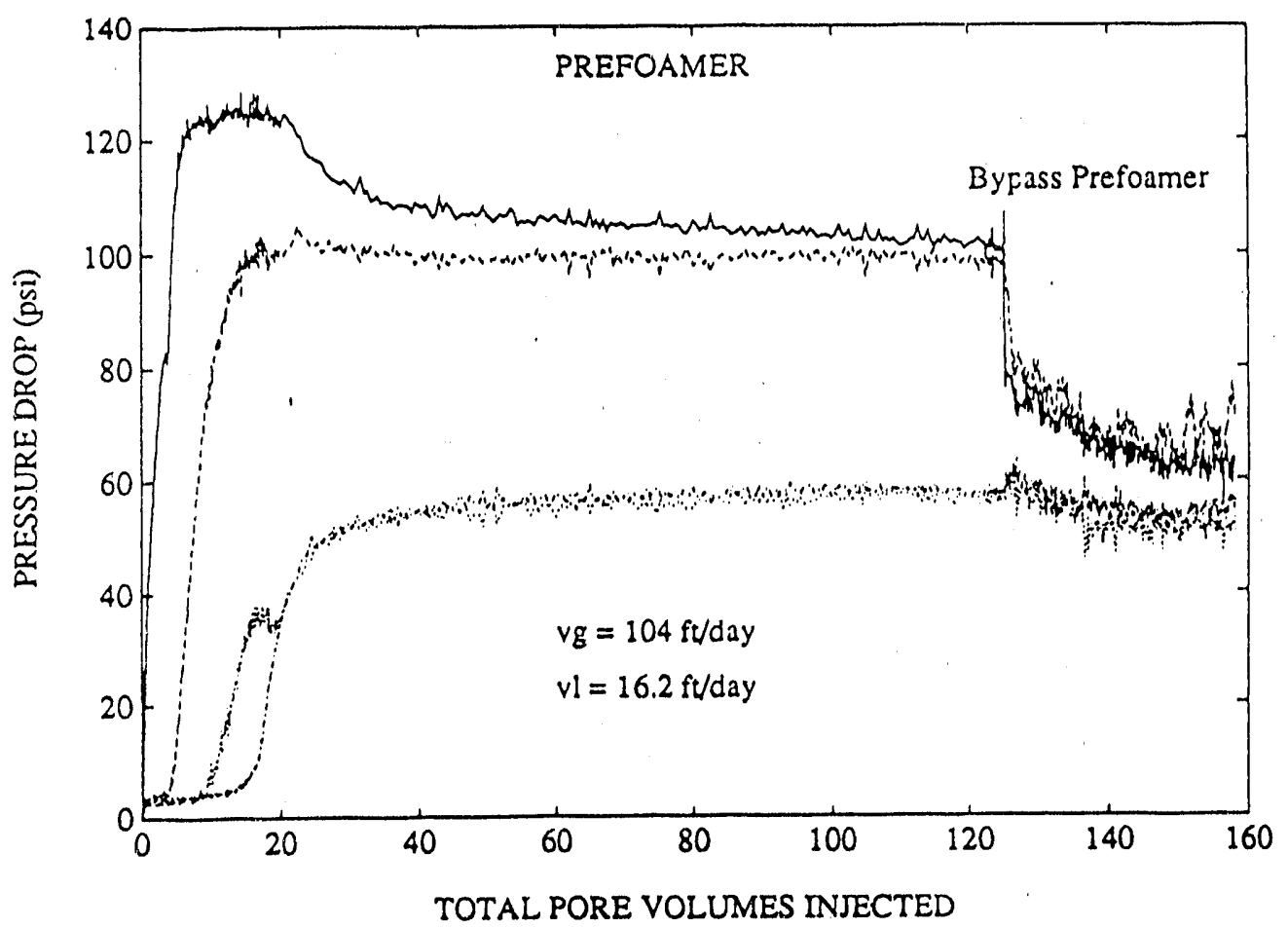

Figure 74: Pressure drop history in Berea sanstone during simultaneous surfactant and gas injection (with prefoamer).

after the prefoamer was bypassed. This is an indication that the effect of the prefoamer does not last beyond a small initial distance of the order of a few $\mathrm{cm}$. Apparently, the porous medium sets the foam texture shortly thereafter.

The same procedure was repeated but without a prefoamer (Figure 75). It was observed that it took more than $50 \mathrm{PVs}$ of injection before the pressure responses stabilized. This volume is more than twice that in the experiment with the prefoamer. In another modification of the experiment, we kept the prefoamer in line for a brief time period only. Shortly thereafter, the bypass line was reopened because of excessive pressures and the liquid velocity decreased by a factor of 2 . Simultaneously, the pressure drop also collapsed by a factor of 2 , indicating Darcy flow for the liquid phase. Detailed analysis of the profile at early times (Figure 76) shows that the pressure increase started first in the second section of the core, indicating that the fluids travel a certain distance before lamellae formation and propagation. The next pressure increase occurred in sections 3 and 4. It is only then that foam started generating in the first section of the core.

Subsequently, the prefoamer was turned on and the inlet pressure experienced a sudden increase (Figure 77). The pressure in the second section of the core increased as well, but to a lesser degree. 


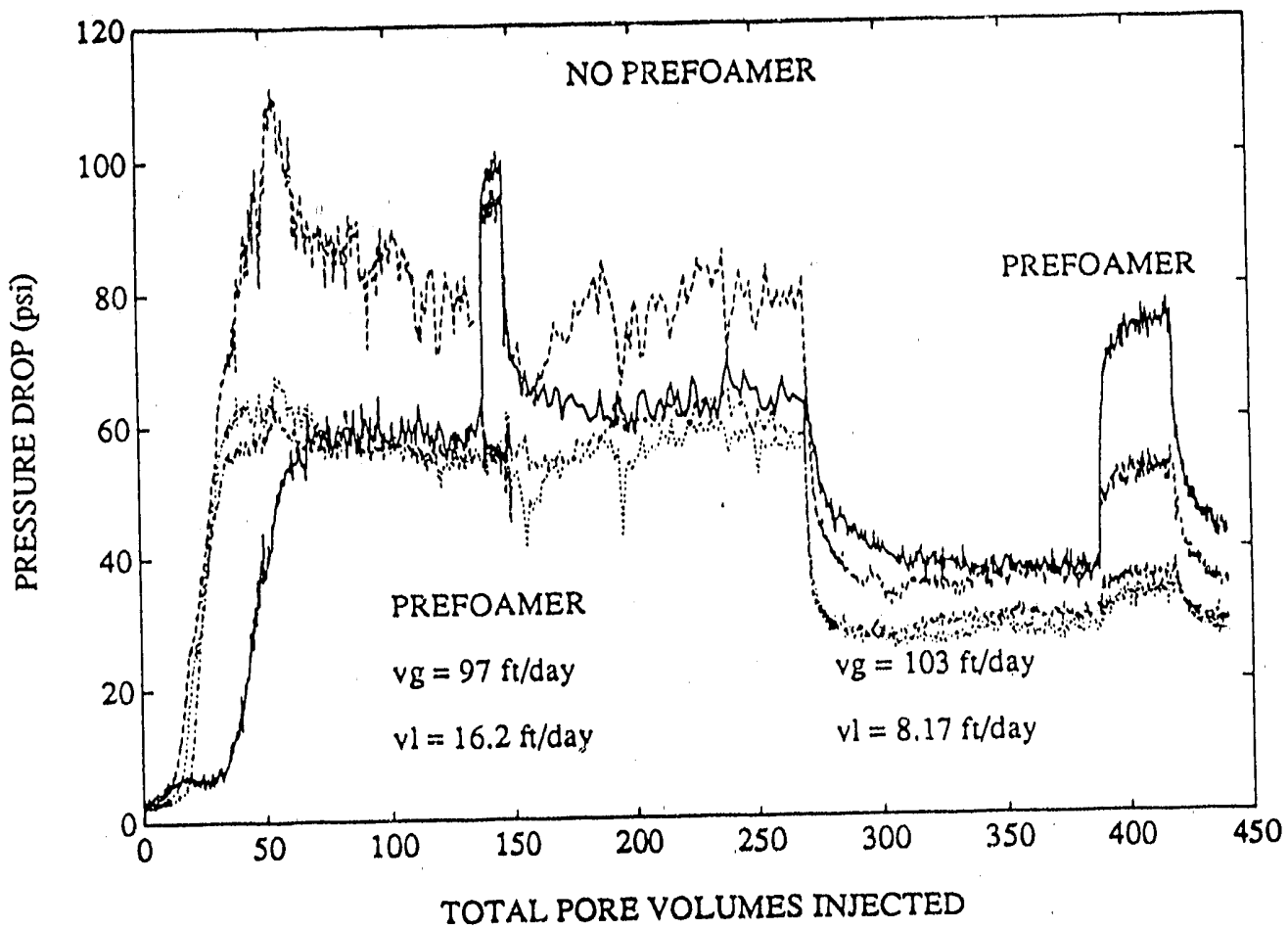

Figure 75: Pressure drop history in Berea sanstone during simultaneous surfactant and gas injection (no prefoamer).

No significant effects were recorded in the third and fourth sections of the core. Bypassing the prefoamer again caused a drop in the pressure in all the sections but most significantly in the inlet end. It was also noted that the pressure decline was slightly slower than the pressure build up. The reason that the last two sections of the core show a slightly higher pressure drop may be the higher gas velocities, which result from higher pressures in the core at the upstream end, which in turn cause higher mass flow rates at constant upstream volumetris flow rates.

Based on the above results, one can conclude that the use of a prefoamer significantly enhances foam flow in porous media by aiding in the generation and propagation of a foam front. It may be also postulated that the foam texture quickly adjusts to the specifications of the porous medium.

The study of the effect of the prefoamer was conducted over a wide range of gas rates at a constant liquid rate. The gas rate was varied stepwise and readings were taken for each gas rate after the pressure drop stabilized. After the curve was generated, the prefoamer was bypassed and the process was repeated. Comparison of the results obtained show that the effect of the prefoamer is more significant at higher gas rates where deviations were higher (Figure 78). The two curves converge at lower gas rates. 


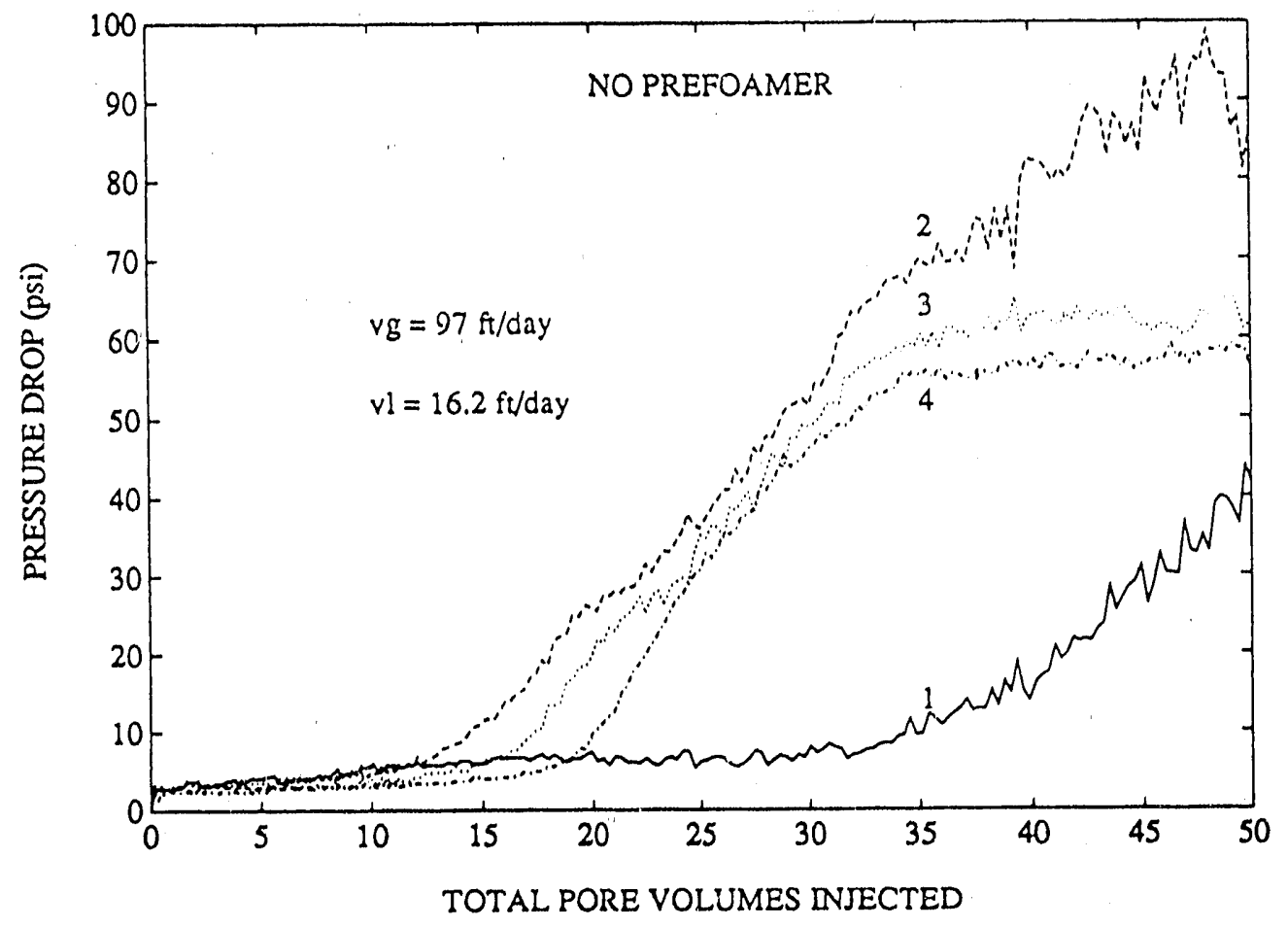

Figure 76: Pressure drop history in Berea sanstone during simultaneous surfactant and gas injection (no prefoamer, early time).

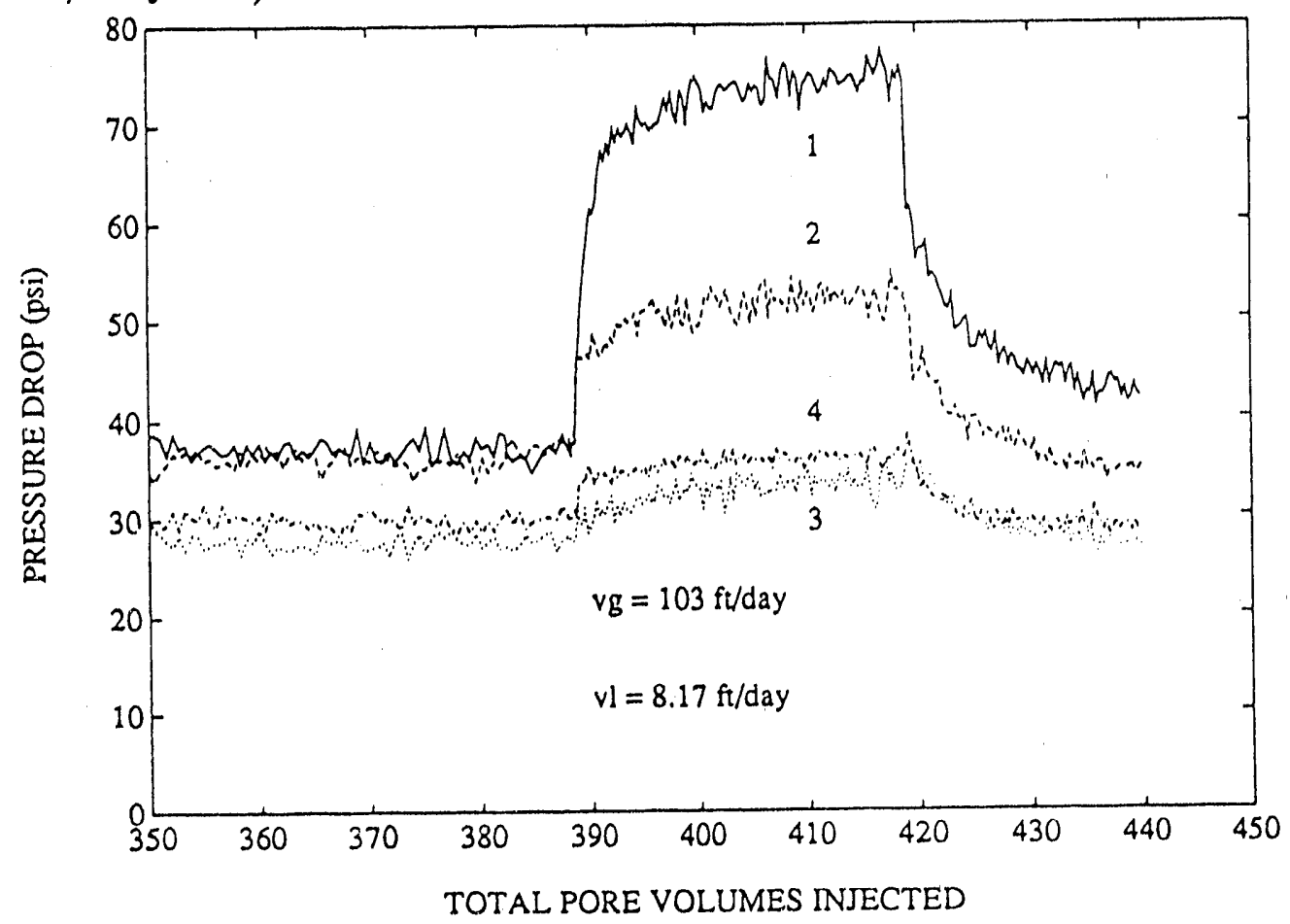

Figure 77: Effect of prefoamer in Berea sanstone during simultaneous surfactant and gas injection. 


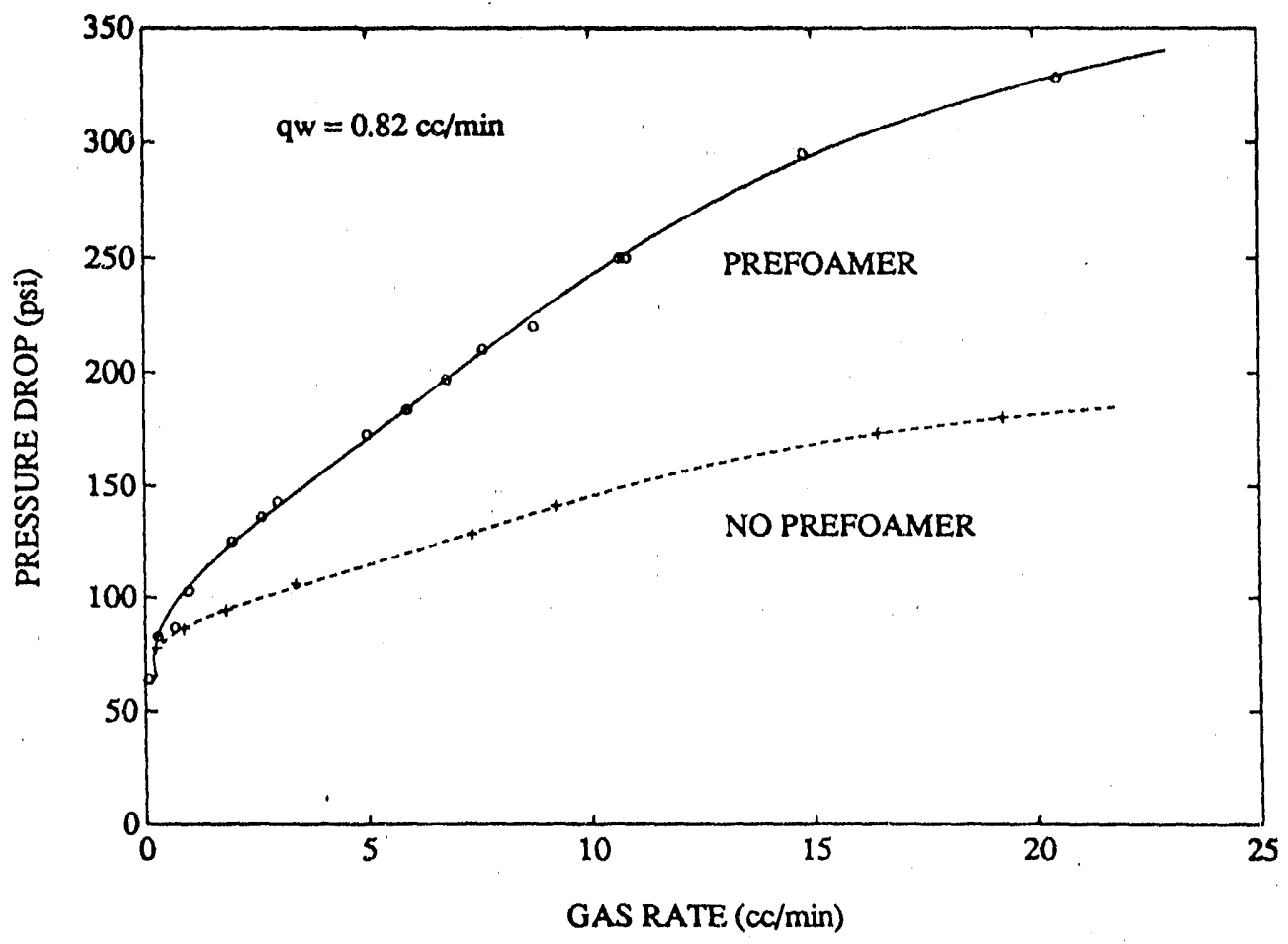

Figure 78: Effect of prefoamer on pressure drop.

The above data were obtained by stepwise changes in the gas rate in either (increasing or decreasing) directions. To investigate hysteresis, the data were next separated. Over the range of rates under investigation, no hysteresis effect was observed (Figure 79) once the higher pressure gradients had been established. This would support the concept of foam flow taking place at fluid saturations that are practically constant.

The same experiment was repeated after resaturating the core with $100 \%$ surfactant solution and then injecting gas and surfactant solution simultaneously until a steady state was achieved. This was followed by stepwise changes in the gas rate and the recording of the steady state data. To differentiate between increasing and decreasing gas rates (Figure 80 ), the data were plotted as two different curves. Once again, no effect of hysteresis of pressure drop on gas rate was discernible, indicating constant saturations in the core independent of the gas rate. It should be noted however, that the absence of hysteresis was observed only after establishing steady state foam flow. Returning to the initial flow conditions before foam was formed did not restore flow without foam.

The results show that the higher pressure drop associated with prefoamer is due to the formation of small bubbles that enter and propagate through the porous medium. However, this effect does not extend far from the inlet end. The effect of the prefoamer is more significant at higher gas 


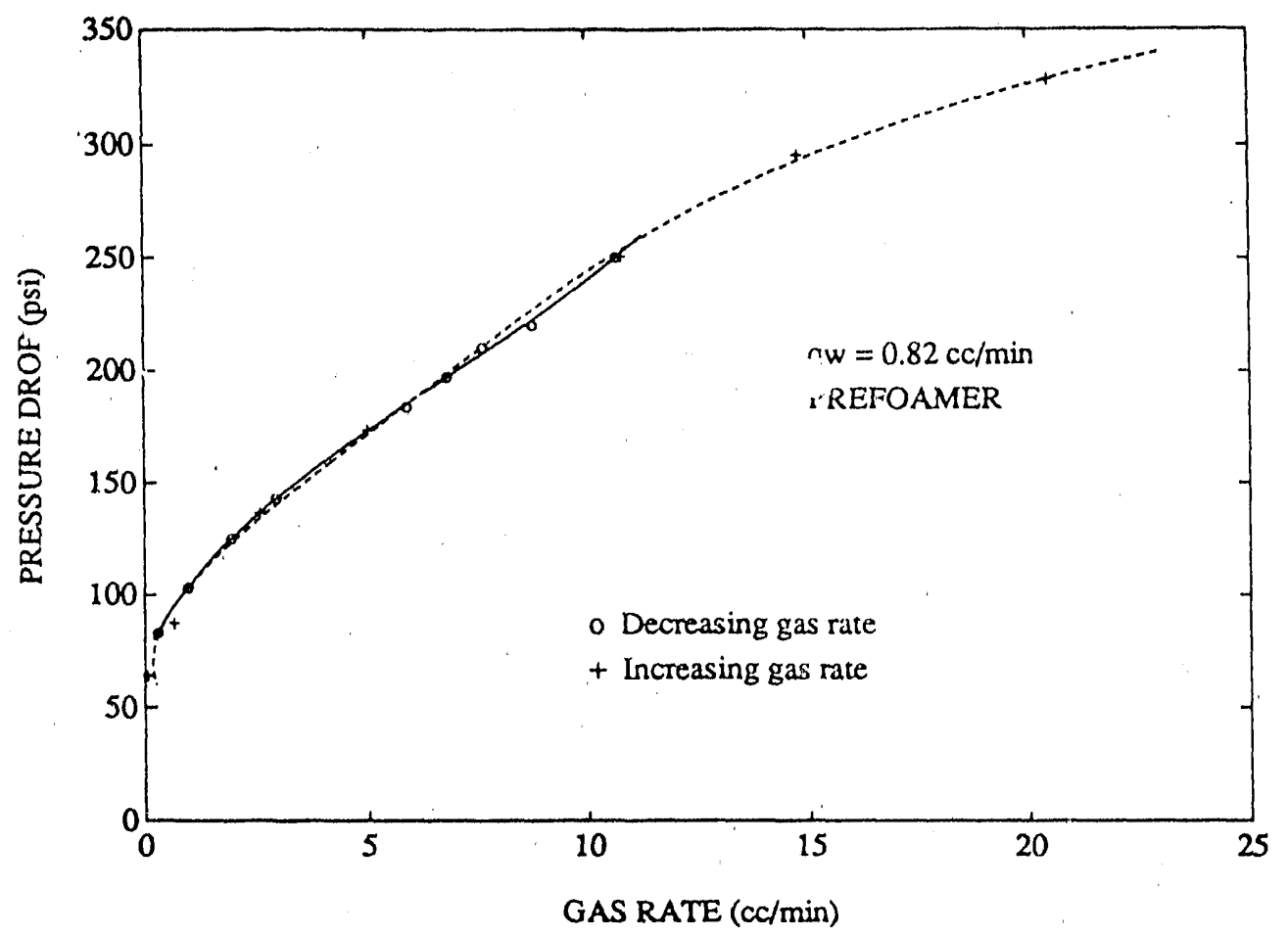

Figure 79: Hysteresis effect of pressure drop on gas rate (with prefoamer).

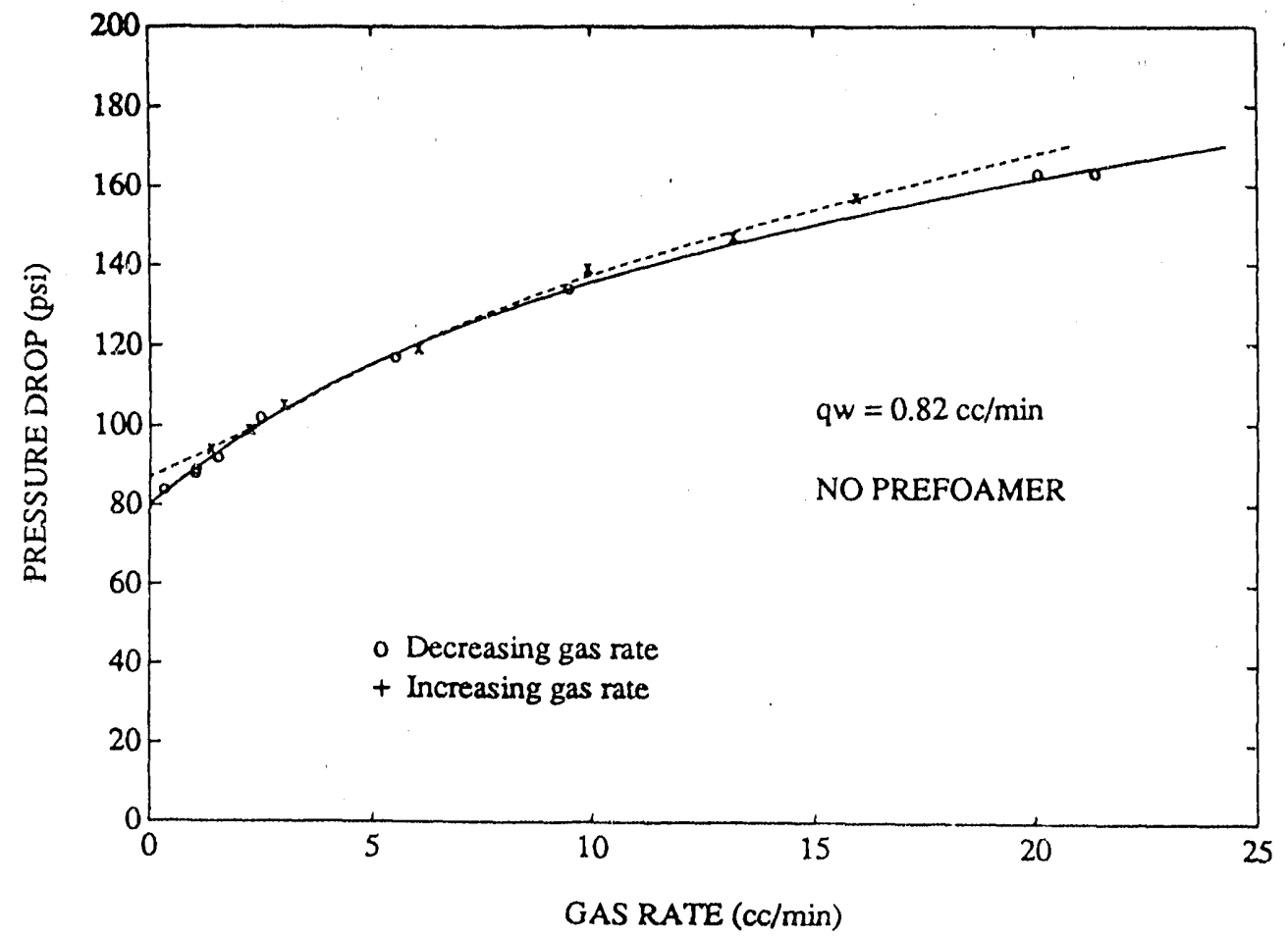

Figure 80: Hysteresis effect of pressure drop on gas rate (no prefoamer). 


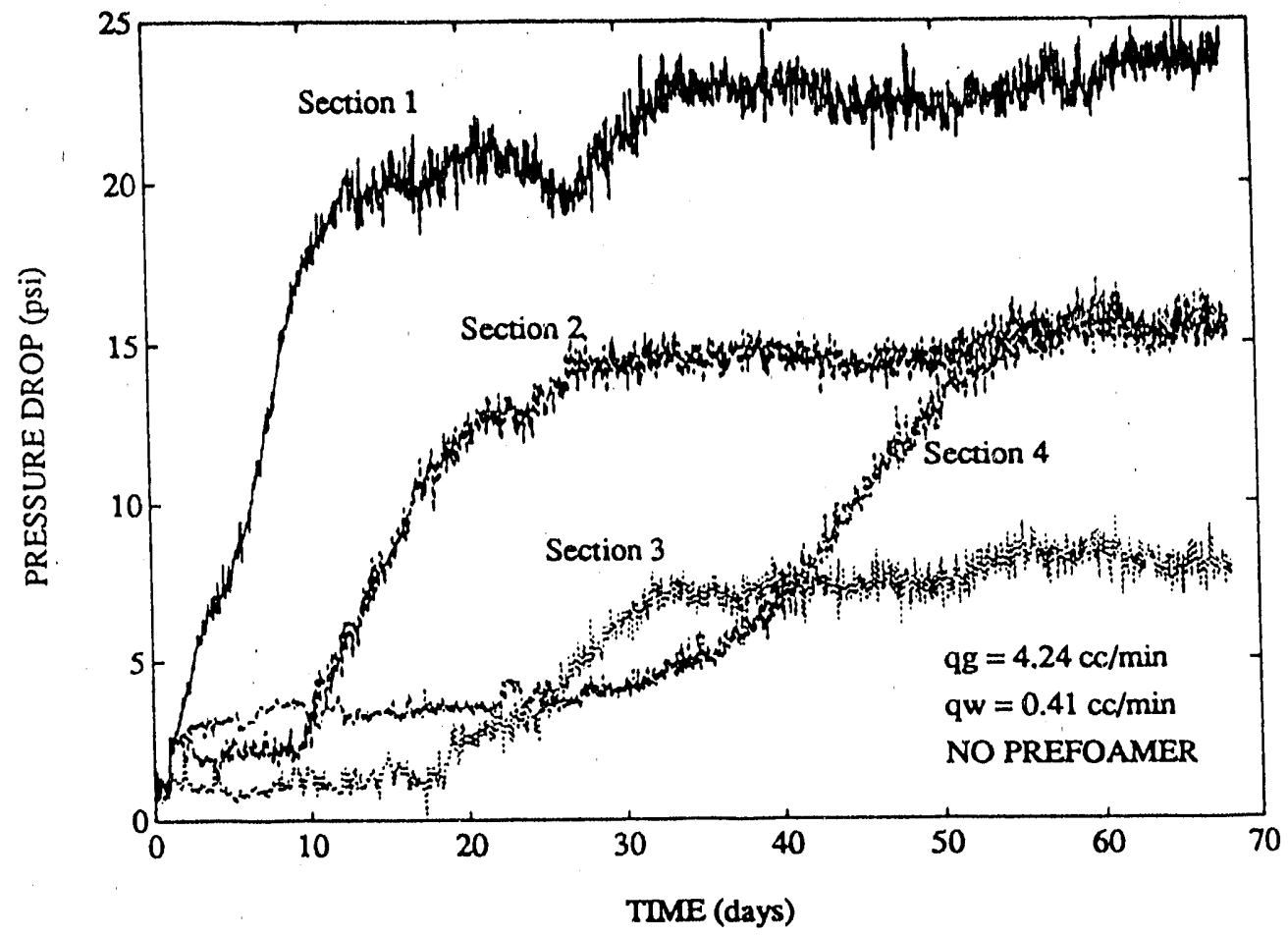

Figure 81: Pressure drop measured across four consecutive core sections (upstream to downstream), as a function of time.

rates. It was also found that due to constant saturations in the core there is no hysteresis effect in the relationship between pressure drop and gas flowrate with or without a prefoamer.

Experiment similar to the previous ones were conducted at surfactant solution flow rates lower than any of the previous. Steady state pressure drops for these particular flow rates were previously measured. However, the former experiments were initiated at higher velocities, first, followed by a rate decrease. A comparison of these data would test the validity of the hypothesis that foam flow in porous media is independent of the direction of change in velocities. Now, the steady state was reached after a period of more than 60 days (Figure 81 ). The steady state reached after the decrease from a high velocity level, and the steady state reached at constant, low rate, are practically identical, the difference in the pressure drops being negligible. This supports the hypothesis that the steady state is independent of the direction from which it is reached, and that the final pressure gradients depend on the magnitude but not on the history of the flow velocities. Again these conclusions about hysteresis apply only after flows has been established in what is characterized as the "strong" foam regime.

A series of steady states were reached at different combinations of gas and surfactant velocities. 
Results are presently being analyzed to describe the effect of individual and cumulative gas and surfactant velocities. 


\title{
9 FLOW OF NON-NEWTONLAN FLUIDS IN POROUS MEDIA.
}

\author{
Y.C. Yortsos
}

\subsection{INTRODUCTION}

The use of non-Newtonian fluids for purposes of mobility control is a routine operation in EOR processes. A variety of rheologically complex fluids are injected to improve sweep efficiencies, divert displacing fluids and block swept zones. In contrast to Newtonian fluids, however, the state of the art in modeling the flow of non-Newtonian fluids, either single- or multi-phase, in porous media is far from complete. As a rule, the interplay of the nonlinear rheology with the porespace geometry results in comlex problems, that prohibit the direct passage from the micro- (pore) to the macroscale (Sorbie et al. [86]). Thus, with few exceptions, laws analogous to Darcy's for flow of non-Newtonian fluids in porous media have not been developed.

Although frequently used in practice, the direct application of single capillary flow expressions to porous media is in principle erroneous. Expressions currently used rely on the inaccurate model of a bundle of parallel capillaries, although more sophisticated approaches have recently appeared (Canella et al. [12], Sorbie [86]). Similar issues arise in the simultaneous flow of a pair of immiscible non-Newtonian fluids in porous media. However, when one or both are of the power-law type, and at conditions of capillary control at the porescale $\left(N_{c a} \ll 1\right)$, the concept of saturation-dependent relative peimeability may be applicable, perhaps in a modified form, to account for possible effects of the power-law rheology. Despite the considerable extent of applications, little is known at present about such dependences.

In this chaptor, we provide approximate solutions to these problems by considering flow in network-like porous media with distributed pore sizes. It is first shown that the success in the averaging of the microscale law for viscous flow relies strictly on the scaling properties (self-similarity) of the power law (of which Newtonian flow is a particular case). This argument is essentially similar to that of Larson [48]. Subsequently, exact analytical results are obtained for the single phase flow of such fluids in networks that lack reconnections. We follow the approaches outlined in Stinchcombe [89] and Heinrichs and Kumar [35]. Two cases are considered, an Effective Medium Approximation at large values of the coordination number $Z$, and a scaling relation near the percolation threshold. Next, we present some applications to the scaling of relative permeabilities near 


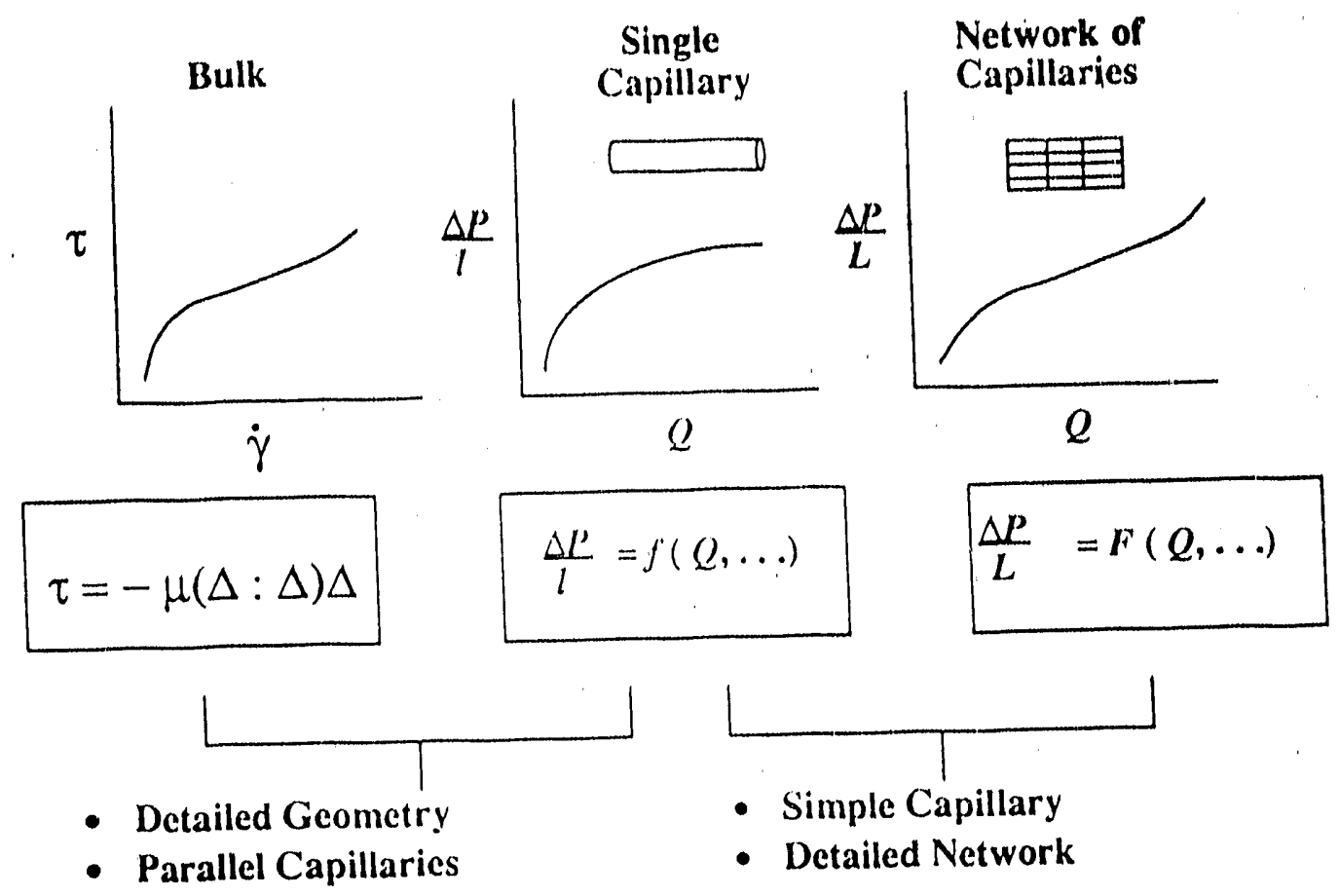

Figure 82: Scale up for flow processes.

the residual saturations. In addition, we extend the critical path approach of Katz and Thompson [40] to postulate a general model for the permeability of a power-law fluid in terms of the formation factor. Finally, two additional results are derived, one for weakly non-linear fluids and another for Bingham plastics.

\subsection{SCALING}

This issue was briefly discussed in [76]. The general question posed is how to average local, single capillary laws for flow over a network of interconnected capillaries. The pore level relationship $\Delta p / l=f(q, \cdots)$ must in general be replaced at the macroscopic level by a different relation $\Delta p / L=$ $F(Q, \cdots)$, capital letters denoting macroscopic variables, and where $F$ is a function different than $f$. A schematic pictorial is sketched in Figure 82. Of course, the problem is not specific to power-law fluids. Non-lirear behavior is displayed by Newtonian fluids at relatively large flow rates (or Re), such that inertia effects become important. For example, this comprises the so-called Forcheimer law for flow of gases (Scheidegger [81]). Non- Newtonian fluids, ranging form power-law to Binham plastics, certainly display such a behavior.

We illustrate the variety of cases by reprinting a figure from Scheidegger showing twelve different 
schematics of non-linear pressure drop-flow rate dependence (Figure 83). A basic result in this subject was obtained in Straley and Kenkel [89], where it was shown that if the pore level relation $f(q)$ is monotonically increasing, there is a unique solution to the macroscopic problem. This facilitates significantly analytical and numerical computations. Clearly, this monotonocity applies to any physical problem involving fluid flow.

It was shown in Larson [48], Kenkel and Straley [41] and Salman et al. [76], that only in the case of the low rate flow of power-law fluids in a two terminal problem (e.g. one injector and one producer), the relationship for a single capillary survives the averaging over the multitude of pores. The reason is that power laws have self-affinity, such that when the flow rate increases by a factor $\lambda$, the corresponding pressure drop increases by the factor $\lambda^{n}$, where $n$ is the power-law exponent. The problem becomes then the determination of the corresponding permeability in terms of the pore microstructure. This subject is discussed in the subsequent sections.

\subsection{POWER-I.AW FLUIDS}

The problem considered in this section involves the flow of power-law fluids in porous media, the latter modeled as a network of capillaries. An important restriction imposed is that such elements are of uniform cross-section, so that the contribution of converging-diverging geometries to the pressure drop is not significant. This may not be necessarily the case in a porous medium, at least for sufficiently high flow rates. Indeed, under such conditions, extensional viscosity effects may add a non-trivial contribution to the pressure drop (Boger [11]). Although recognized, this subject will not be discussed here. Equivalently, this section addresses the problem only at low flow rates.

Let the porescale expression for the power-law fluid with exponent $n$ be of the form

$$
q=v(\Delta p)^{1 / n}
$$

where $q$ is the volume flow rate across a pore, of conductance $v$, with applied pressure drop $\Delta p$. Under the above qualifications, which render the problem into one of power-law resistors, the following aspects will be addressed: (i) Effective Medium Averages for Bethe Lattices; (ii) Behavior near Percolation; and (iii) Critical Path Analysis for Permeability. 

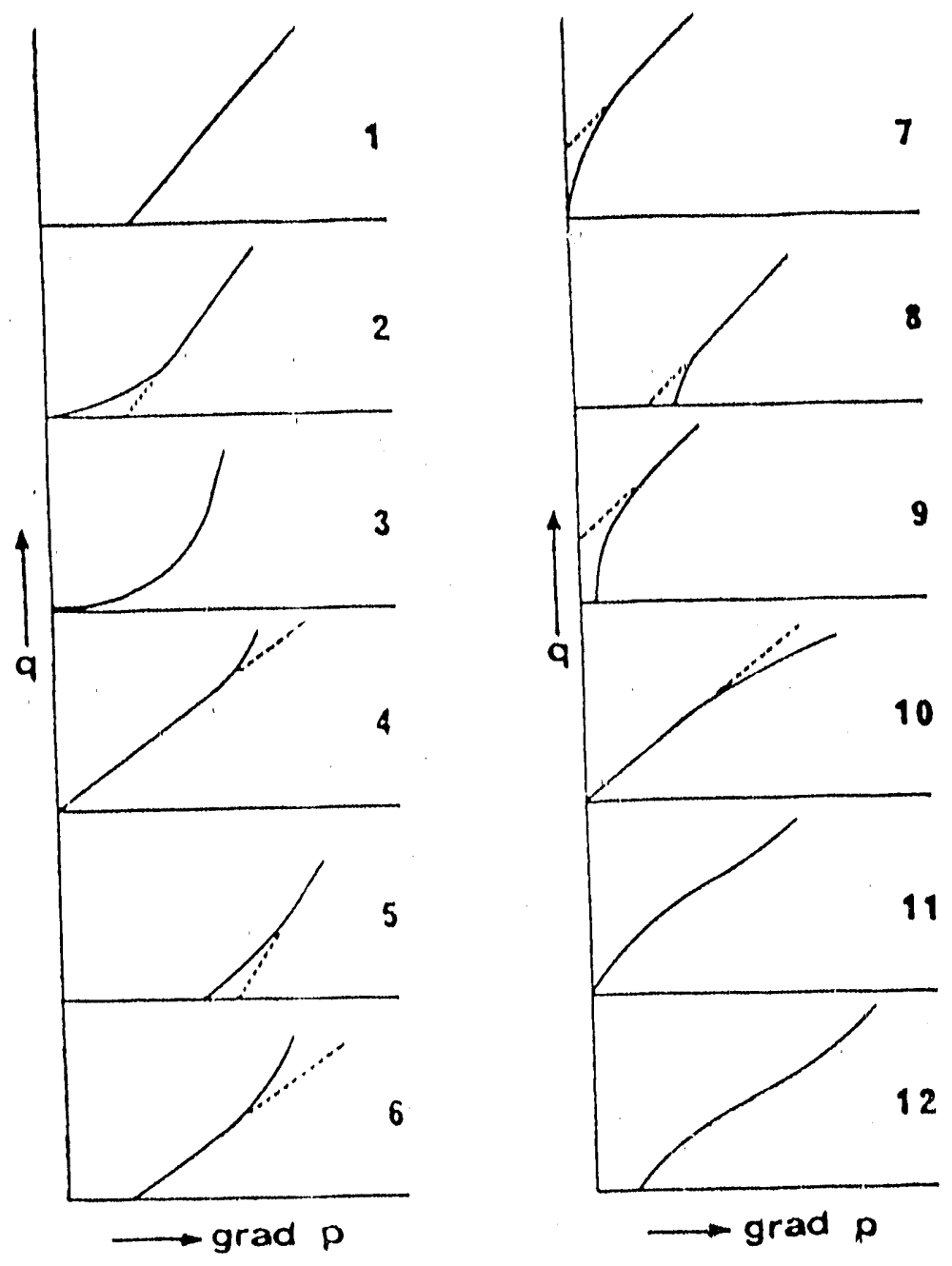

Figure 83: Schematics of non-linear pressure drop-flow rate relations (from Scheidegger). 

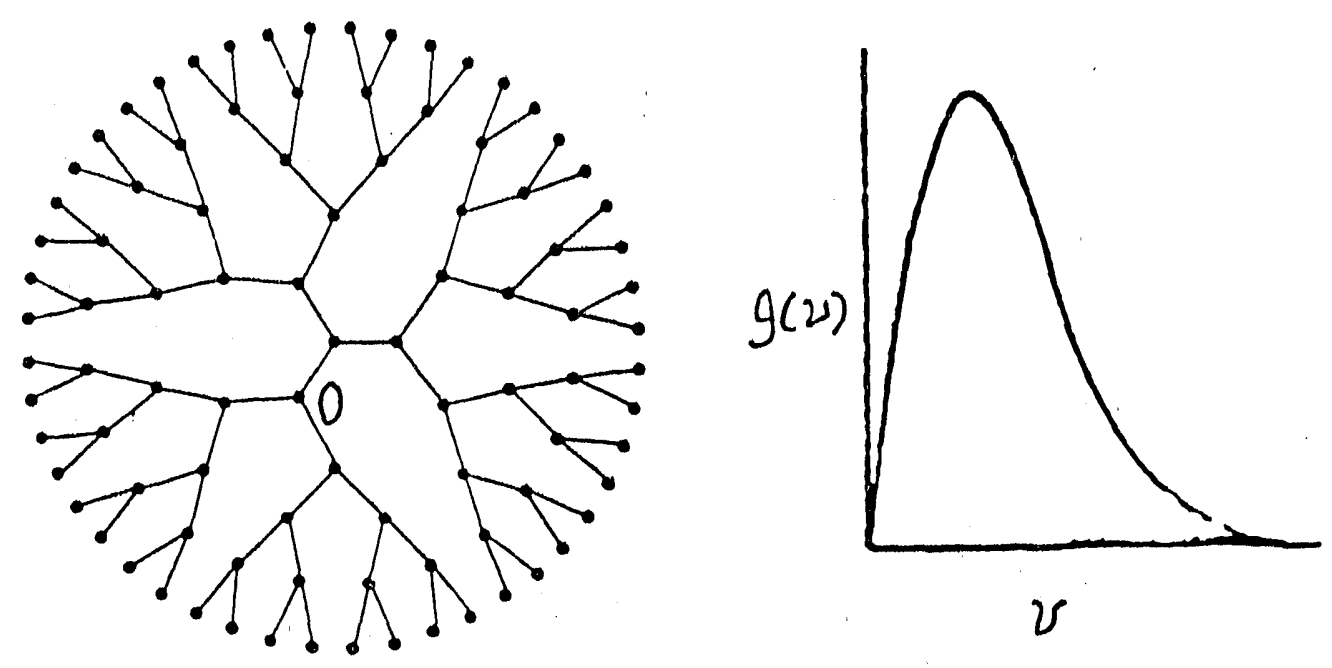

Figure 84: Schematic of a Bethe Lattice of $z=3$ and a conductance distribution

\subsubsection{Effective Medium A verages}

Bethe lattices lack interconnections, hence they are most suitable for analytical calculations (Figure 84). We proceed by assuming that the conductance $v$ is distributed, e.g. $v \sim r^{3+1 / n}$, where $r$ is the pore radius (Figure 84 ). The problem posed is to find the conductance from site $O$ to infinity. Obviously, we have

$$
V_{0}=\sum_{i}^{z-1}\left[\frac{1}{v_{i}^{n}}+\frac{1}{V_{i}^{n}}\right]^{-1 / n}
$$

To proceed, we follow a self-consistency approach, as in Stinchcombe [88] and Heinrichs and Kumar [35]. All details, omitted here for simplicity, will be presented elsewhere. In particular, we consider the case of large values of the coordination number $Z$. This limit yields in the Newtonian case the correct expression for the Effective Medium Approximation, thus it may be considered its power-law analogue. The leading order term in the expression obtained reads

$$
\int_{0}^{\infty} g(v)\left[\frac{(z-1) v}{v^{n}+\left(\left((z-1)^{n}-1\right) v_{m}^{n}\right)^{1 / n}}-1\right] d v=0
$$

This is an integral equation for $v_{m}$, the solution of which allows for the average and total conduc- 
tances $\langle v\rangle$ and $\left\langle v_{T}\right\rangle$, respectively, to be evaluated

$$
<v>=\left((z-1)^{n}-1\right)^{1 / n} v_{m} ;<v_{T}>=\frac{z}{(z-1)}<v>
$$

Recall that the equivalent result for the linear (Newtonian fluid) case is (Stinchcombe [88])

$$
\int_{0}^{\infty} g(v) \frac{\left[v-v_{m}\right]}{v+(z-2) v_{m}} d v=0
$$

which is of course obtained from the above in the appropriate linit $(n=1)$. The corresponding expression for regular lattices is given in Kirkpatrick [44]

$$
\int_{0}^{\infty} g(v) \frac{\left[v-v_{m}\right]}{v+\left(\frac{z}{2}-1\right) v_{m}} d v=0
$$

Using largely qualitative arguments, the following expression was obtained by Canella et al. [12]

$$
\int_{0}^{\infty} g(v)\left[\left(\frac{\frac{z}{2} v_{m}}{v+\left(\frac{z}{2}-1\right) v_{m}}\right)^{n}-1\right] d v=0
$$

which was claimed to be an EMA expression for power-law fluids. At this point, rigorous results for EMA in general networks have not been obtained, to our knowledge, hence the validity of this conjecture is questionable. Our Bethe lattice result is certainly rigorous and perhaps more appropriate, although of somewhat limited applicability. In any case, a comparison of our results for Bethe lattices to the above shows the subtlety involved when power-law behavior is introduced. We also note that in the special cases of parallel capillaries $(Z=\infty)$ and capillaries. in series $(Z=2)$, we obtain arithmetic and harmonic averages, respectively

$$
\begin{gathered}
u_{m}=\int_{0}^{\infty} v g(v) d v \\
u_{m}=\left[\int_{0}^{\infty} g(v) u^{-n} d v\right]^{1 / n}
\end{gathered}
$$

All these results are valid away from percolation.

\subsubsection{Behavior Near Percolation}

Near percolation, the behavior is quite different. Scaling laws have previously been derived for Bethe and regular lattices. We shall take the following distribution 


$$
g(v)=p \propto(v)+(1-p) \delta(v)
$$

and consider the limit as the percolation threshold, $p_{c}$, is approached. Omitting considerable details, we have found the following result for Bethe lattices

$$
v_{m}=\frac{2 p_{c}}{\left(1-p_{c}\right)}\left(-\phi^{\prime}(0)\right) G^{1 / n}\left(\frac{p-p_{c}}{p_{c}}\right)^{1+\frac{2}{n}}\left(\frac{1}{1-p_{c}^{n}}\right)^{1 / n}
$$

where

$$
G=\frac{n \Gamma(N-n-1)}{\int_{0}^{\infty} \alpha(\vartheta) v^{n} d v} ; N=[n]+2
$$

$\Gamma(z)$ is the Gamma function and $[n]$ is the integer part of $n$. Obviously, for shear-thinning fluids, $N=2$. The function $\phi(x)$ satisfies the following integro-differential equation

$$
(-1)^{N} x \int_{0}^{\infty} \frac{\phi^{(N)}(x+\rho) d \rho}{\rho^{n+2-N}}=\phi(1-\phi)
$$

subject to the conditions $\phi(0)=1, \phi(\infty)=0$. Upon application of a Weyl transform, we may also recist this equation into

$$
\phi^{(N-1)}=\frac{(-1)^{N-1} \sin \pi(N-n-1)}{\pi} \int_{x}^{\infty} \frac{\phi(t)(1-\phi(t)) d t}{t(t-x)^{1-n}}
$$

The above equation is a generalized form of the Stinchcombe result for the linear case $(n=1)$

$$
x \phi^{\prime \prime}=\phi(1-\phi)
$$

We are currently studying the solution properties of the integrodifferentisl equation.

For general lattices, results near the percolation threshold have been known from the work of Straley and Kenkel [90]. The conductivity scaling is of the form

$$
v_{e f f} \sim\left(p-p_{c}\right)^{t_{n}} \quad ; \quad t_{n} \simeq(d-1) \mu+\frac{\zeta(n)-\nu}{n}
$$

where the parameter $\zeta(n)$ is slowly varying, the exponent $\mu$ pertains to the correlation length ([87]), :nd $d$ is the dimension of the system. For instance, for a 3-d network, we shall obtain 


$$
t_{n} \simeq 1.76+\frac{0.24}{n}
$$

The above expressions can be applied to either single- or multi-phase permeabilities. The latter require that the occupancy of the pore space by the respective phases is known, a condition best satistied under capillary control. Relative permeabilities may be readily constructed. For example, we can determine the scaling of relative permeability curves for the flow of a non-Newtonian fluid near the various thresholds. For this, we combine the approach outlined in Sahimi and Yortsos (1990) with the above expressions. Near the primary drainage threshold, we have

$$
S_{n N} \sim\left(p-p_{c}\right)^{\beta}
$$

and

$$
k_{r n N} \sim v^{n} \sim\left(p-p_{c}\right)^{n t_{n}}
$$

Therefore, we obtain

$$
k_{r n N} \sim S_{n N}^{\frac{n t_{n}}{\beta}} \sim S_{n N}^{0.558+4.08 n}
$$

where subscript $n N$ stands for non-Newtonian. This yields the scaling of the non-wetting phase in the 3 - $d$ case. The strong dependence of the exponent on the index $n$ should be noted. Near the trapping treshold, on the other hand,

$$
S_{n N}-S_{r} \sim\left(p-p_{c}\right)^{1+\beta}
$$

Therefore, we obtain

$$
k_{r n N} \sim\left(p-p_{c}\right)^{\frac{n t_{n}}{1+\beta}} \sim\left(S_{n N}-S_{r}\right)^{0.167+1.227 n}
$$

Both the above show that the relative permeabilities are flatter for more shear-thickenning fluids. Knowledge of this behavior is useful for displacement processes, where the typical fractional flow function depends on both the rate and the power-law exponent (Salman et al. [77]). 


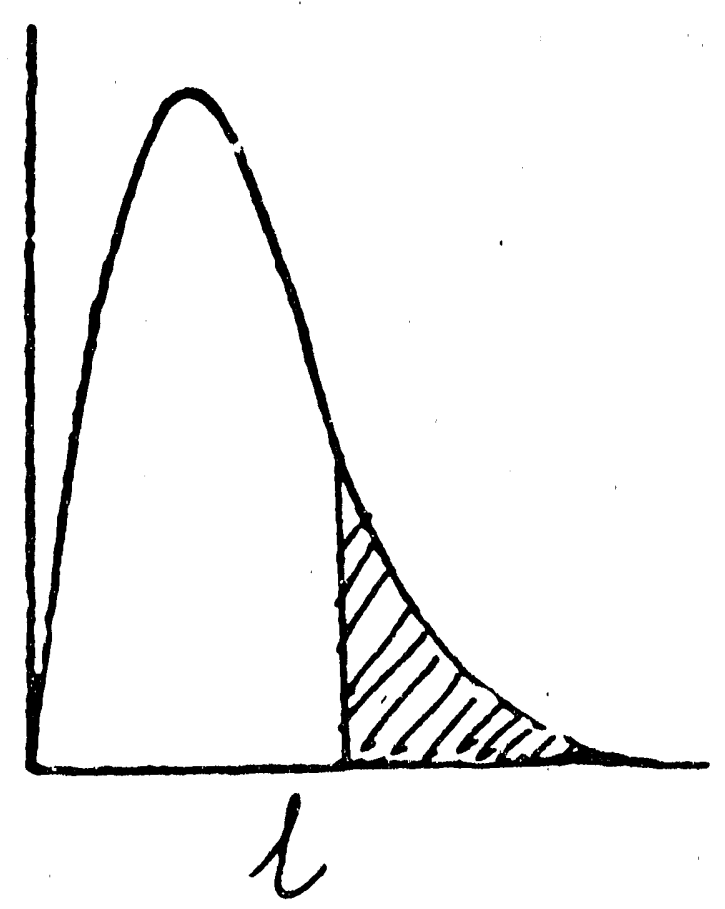

Figure 85: Schematic of conductance distribution.

\subsubsection{Critical Path Analysis}

Next, we shall extend the method of Katz and Thompson [40] for the derivation of a macroscopic law for power-law fluids. The individual pore conductances for power-law fluids are given by

$$
Q=v(\Delta P)^{1 / n}
$$

where

$$
v=c_{n} l^{3} ; c_{n}=\frac{n \pi}{(1+3 n)} \frac{1}{2 m}^{1 / n}
$$

and $l$ is the characteristic pore size. Following [40], we consider the size distribution $p(l)$ and maximize the sample conductance (Figure 85)

$$
v(l) \propto l^{3}\left[p(l)-p_{c}\right]^{t_{n}}
$$

to find the result

$$
l_{m a x, n}=l_{c}\left[\frac{3}{3+t_{n}}\right]
$$


In the above, the characteristic length $l_{c}$ solves the equation

$$
p\left(l_{c}\right)=p_{c}
$$

It must be noted that the above is expected to lose accuracy as $n$ approaches 0 . Upon substitution, we find the general result for the flow of a power-law fluid in a core

$$
q^{n}=\frac{k_{1} \Delta P A k_{1}^{\frac{n-1}{2}}}{m L}
$$

where

$$
A=\left[\left(\frac{\sigma}{\sigma_{0}}\right)^{0.38} \phi^{0.12}\right]^{n-1} \frac{(7.84)^{1-n} 3^{1+n}\left(23.57 t_{n}\right)^{t_{n}}}{2^{2 n+1}(1+3 n)^{n}\left(3+t_{n}\right)^{2+t_{n}}}
$$

In the above, $k_{1}$ is the Newtonian permeability, $\sigma_{0} / \sigma=F$, where $F$ is the formation factor, and we have used the relationship

$$
k_{1}=\frac{1}{226} l_{c}^{2} \frac{\sigma}{\sigma_{0}}
$$

Note that this is exact. We may also substitute an expression for the formation factor in terms of porosity (see Katz and Thompson [40]). The above relation gives explicitly the extension of Darcy's law for the flow of power-law fluids in terms of porous media and fluid properties. For comparison we present related results by Christopher and Middleman [17]

$$
A=12\left(\frac{n}{3+9 n}\right)^{n}(150 \phi)^{\frac{(n-1)}{2}}
$$

and by Teeuw and Hesselnik [92]

$$
A=\frac{1}{2}\left(\frac{n \phi}{3 n+1}\right)^{n}\left(\frac{8}{\phi}\right)^{\frac{n+1}{2}}
$$

All these results were derived for power-law fluids. We shall conclude this chapter with two related applications, one for weakly non-linear and another for a Bingham plastic. 

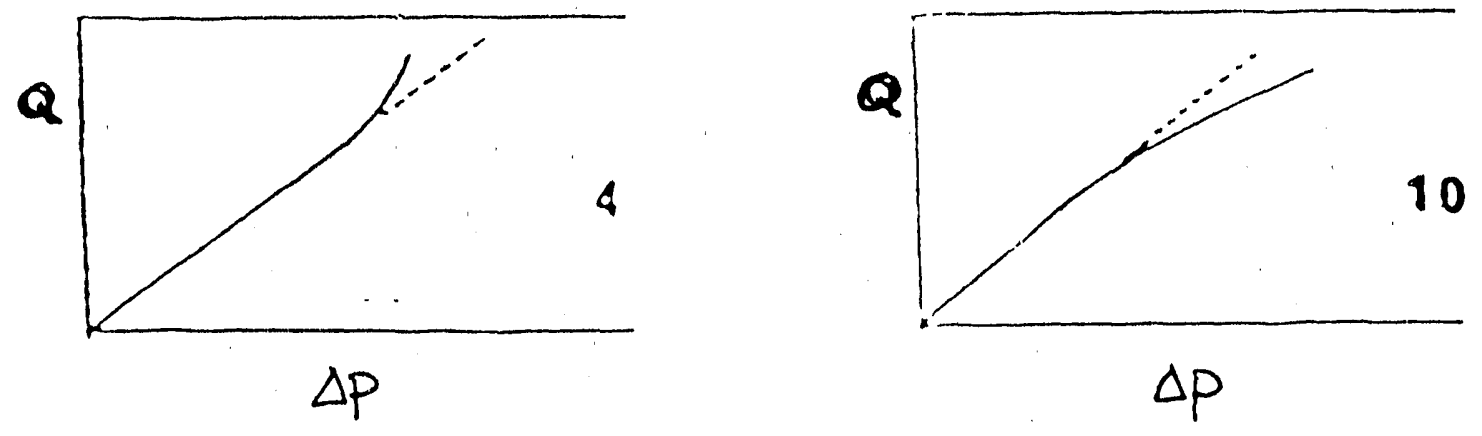

Figure 86: Schematic of flow rate-pressure drop relation in the Forcheimer regime.

\subsection{WEAKLY NON-LINEAR FLUIDS}

We consider the case of weakly non-linear fluids. This, for instance, can be the flow of a Newtonian fluid at not too small Reynolds numbers, as in the Forcheimer regime (Figure 86). For our purposes, we shall assume that at the pore level the following holds

$$
Q_{i}=g_{i}(\Delta P)+\epsilon \sigma_{i}(\Delta p)^{2}
$$

where, parameter $\epsilon$ is small. We derive results based on EMA for Bethe lattices. The following is obtained

$$
Q=\langle g\rangle(\Delta p)+\epsilon\langle\sigma\rangle(\Delta p)^{2}+\cdots
$$

where $\langle g\rangle$ is the same average as in the linear case

$$
\int_{0}^{\infty} G(g)\left(\frac{g\langle g\rangle}{g+\langle g\rangle}-\frac{\langle g\rangle}{z-1}\right) d g=0
$$

The novel result is in $\langle\sigma\rangle$ which satisfies the following equation, if $g$ and $\sigma$ are uncorrelated 


$$
\langle\sigma\rangle=\langle g\rangle^{3} \frac{\int_{0}^{\infty} \frac{G(g) d g}{(g+\langle g))^{s}} \int_{0}^{\infty} \sigma \Sigma(\sigma) d \sigma}{\left(p_{c}-\int_{0}^{\infty} \frac{g^{3} G(g) d g}{(g+\langle g\rangle)^{3}}\right)}
$$

In the case of correlation, the two integrals combine into a double integral. This expression can be used for the evaluation of the Forcheimer coefficients in terms of the microstructure. Further work is in progress.

\subsection{A NOTE ON BINGHAM PLASTICS}

We close this Chapter by presenting a note on the mobilization of Bingham plastics. Bingham plastics have been proposed as rnodels for the rheological ehavior of heavy oils. It is well known that for Bingham plastics to flow, a yield stress $\tau_{0}$ must be exceeded. The rheology of Bingham plastic flow in a porous medium is still not fully resolved. However, a certain result can be obtained regarding its non-flowing saturation, $S_{n f}$. The latter is defined as the minimum saturation value, below which no flow (mobilization) is possible. Clearly, $S_{n f}>S_{\text {or }}$, where $S_{\text {or }}$ is the familiar residual saturation value. Therefore, without loss, we shall take

$$
S_{n f}-S_{o r} \sim p-p_{c}
$$

where $p_{\mathrm{c}}$ is the percolation threshold.

Consider the problem, where a Newtonian fluid flows in a porous medium at rates such that the Bingham plastic is at the threshold of being mobilized (Figure 87). The corresponding pressure drop required is

$$
\Delta p \sim\left(\frac{L}{r_{0}}\right)^{6} \tau_{0}
$$

where $\zeta$ is the chemical distance exponent and $L$ is the distance over which the pressure drop is applied. Assuming that we are near percolation, the length $L$ is similar to the correlation length

$$
L \sim\left(p-p_{c}\right)^{\mu} r_{o}
$$

The pressure drop is due to the flow of the Newtonian fiud, thus

$$
\frac{\Delta p}{L} \sim \frac{q_{N} \mu_{N}}{k_{N}}
$$




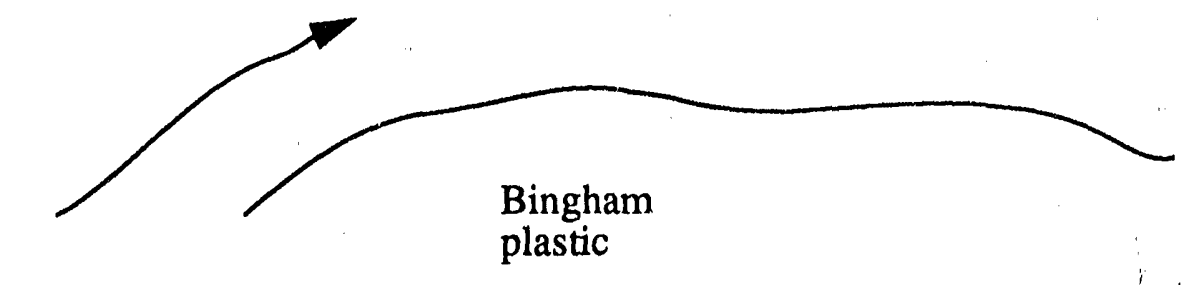

q

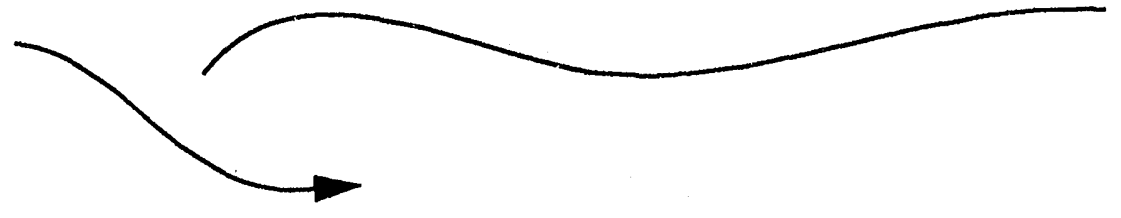

Newtonian

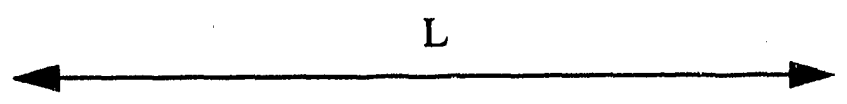

Figure 87: Schematic of Bingham plastic mobilization.

By combining the above we finally arrive at the result

$$
S_{n f}-S_{o r} \sim\left(\frac{q_{N} \mu_{N} r_{o}}{\tau_{o} k_{N}}\right)^{-\frac{1}{\mu(\delta-1)}}
$$

We can approximate the typical size $r_{0}$ by the permeability, $r_{0}=\sqrt{k}$, to yield

$$
S_{n f}-S_{o r} \sim\left(\frac{q \mu}{\tau_{o} \sqrt{k}}\right)^{-\frac{1}{\mu(\zeta-1)}}
$$

This result shows the dependence of the non-flowing saturation $S_{n f}$ on the flow rate. This dependence is very strong, the exponent being equal to -5 for typical 3-d values. Further work in this area is currently being conducted. 


\section{SUMMARY AND FUTURE WORK}

Advances have been made in the areas of (i) Vapor-Liquid Flow in Porous Media, (ii) Reservoir Heterogeneity, and (iii) Chemical Additives. A total of eight separate investigations were carried out in the above areas using theory, numerical computation and experiments.

Vapor-liquid flow in porous media was studied from three different angles. One involved numerical modeling in pore networks. A second investigation dealt with the visualization of steam displacement processes in Hele-Shaw cells and glass micromodels. While, in a third study we considered macroscopic modeling of steady-state steam-water flows in porous media. The latter is commonly used to determine steam-water relative permeabilities. The results of all these investigations have been valuable.

In our pore network analysis of steam-water systems in porous media we made substantial progress in understanding the role of superheat, the effects of various forces, and the influence of the pore structure on the growth of vapor bubbles in porous media. We have modeled the process using percolation theory concepts, including gradient percolation, and have identified the conditions under which they are applicable. Our ultimate goal is to develop pore network prototypes for the process of steam injection and for other vapor-liquid flows (such as steady-state countercurrent and concurrent flows). Although progress has been made, significant work still needs to be done. Currently, we are investigating methods to include heat transfer, particularly the conduction of heat through the solid matrix. The end product of this project would be the development of expressions for relative permeabilities in vapor-liquid flows under various conditions.

The use of flow visualization for steam injection in Hele-Shaw cells and in glass micromodels has been very productive, despite the lack of control for heat transfer. In Hele-Shaw cells, we concentrated on the steam front dynamics, which have been found to be substantially different from the typical immiscible interface. Additional phenomena involving steam distillation and the nonNewtonian response of heavy oils were identified. In glass micromodels, we examined trapped oil displacement from pore doublets and dead-end pores. We have discussed the dominant mechanisms observed, including film and roughness flows. We plan further experiments in both these directions. Our approach will be to isolate key observed mechanisms, and to proceed with a comprehensive study. We are experimenting also with methods to control heat transfer. A particularly interesting experiment to be carried out involves the study of countercurrent steam flow in glass micromodels. 
This process can be taken to represent steamflooding in naturally fractured systems.

Our work in the macroscopic modeling of steady-state vapor-liquid flows in porous media was aimed at the effect of rate and core length on saturation profiles, thus on the accuracy of various methods for the determination of vapor-liquid relative permeabilities. It was found that there exists a plateau in the (modified) macroscopic capillary number values, such that saturation profiles are flat throughout most of the core. Operating in that region optimizes the accuracy of relative permeability estimation for a vapor-liquid system. Future work on macroscopic modeling of vaporliquid flows involves a thorough analysis of the effects of permeability heterogeneity (end effects). We are also planning the modeling of 2-D flows, such as the steam chamber in steam injection in horizontal wells.

Effects of reservoir heterogeneity were addressed in three different studies. One important aspect involved the representation of fractured systems, particularly the network of fractures. Another stu.dy addressed how to average on a large scale, an issue of great importance to coarse-grid simulation. Finally, we have made progress in analyzing regimes in parallel flow, a condition often encountered in typical geological settings.

We have launched an effort to apply fractal geometry concepts for the characterization of the network of fractures in naturally fratcured systems. In addition to providing a great flexibility for the synthesis of various patterns, fractal networks have indeed been identified in many field cases. Key issues in this area include the numerical representation of the major fractal features of the network, the connection to the fracturing process itself, the simulation of displacement processes in such networks, and the large-scale extrapolation of the results obtained. We have made progress in some of the above issues. A numerical technique was developed for the synthesis of fractal networks of fractures and steady-state single phase flow was simulated. Future work would continue along these lines. In addition, near term plans involve the simulation of steam injection and flow processes in such networks.

We made significant advances regarding the large scale averaging of simple processes, such as primary drainage. Our approach to this non-trivial problem vras to apply pore-network methodologies to the macrosopic heterogeneity problem. The first problem that we have considered assumes capillary control at the local scale, so that we may proceed with a local, rather than a non-local, formalism. Plans are also pursued along the latter direction, which encompasses the general case. 
We have found that large- scale heterogeneity leads to additional trapping of residual phases and that it makes a system appear more strognly wet, from a macroscopic point of view. During this process, we were able to improve on the a previous technique of percolation with trapping, for a large-scale implementation. Future plaris include the large scale averaging of imbibition.

We have also considered the issue of parallel flow in porous media. By the latter, we refer to flow in a long and narrow geometry, possibly heterogeneous. Such conditions are often encountered in practical applications. One relevant question, here, is the interface dynamics. Such a study has been undertaken for Hele-Shaw geometries. Theory, simulation and experiments were combined to show how viscous coupling operates in these flows. We have found that such interfaces support wave motion, the dynamics of which, in certain cases, are governed by the well known Kdv equation. The latter predicts the formation of solitons, which have played an important role in non-linear wave propagation in many areas of physics and engineering. We are currently planning to extend our approach to actual porous media at both the microscopic and the macroscopic levels.

Finally, the third component of research reported involves the use of chemical additives for the improvement of oil recoveries. Part of the past activity was devoted to the study of alkaline flooding at elevated temperatures. That work has been completed and we are hoping that a final report will become available soon. Another effort has been in the experimental study of foam flow in laboratory cores. In addition, we have started an investigation on the modeling of non-Newtonian flow in porsius media.

Experimental work on foams centered on the issues of foam generation and foam propagation under the steady-state injection of surfactant solution and gas. Various flow conditions were examined. The formation of weak and strong foam was delineated. In our experiments, it was found that weak foam precedes strong foam, the formation of which required an incubation period. The propagation of strong foam into the porous medium was monitored by in-situ pressure monitoring. We are analyzing currently available theories to understand the issues of strong foam formation and propagation. It appears that further theoretical study would be necessary for a satisfactory resolution of these issues.

The final topic of this report addressed non-Newtonian flow in porous media. We have focused our effort in deriving macroscopic (Darcy law-type) descriptiors based on pore level laws. As expected, although not properly emphasized in the ljterature, only a small class of non-Newtonian 
fluids, namely power-law fluids at low rates, is amenable to a relatively simple approach. We proposed extensions of Effective Medium Theories and Percolation methods for the derivation of macroscopic flow rate-pressure drop relations. Some aspects of Bingham plastic flow, which is related to heavy oil mobilization, were also discussed. Work on this vast subject continues. Presently, we are studying extensional flow effects on the overall pressure drop of a power-law fluid. 


\section{PUBLICATIONS}

The following publications have resulted from this research during the period reported:

1. Yortsos, Y. C., and Zeybek, M., "Long Waves on Hele-Shaw Fingers," paper presented at the 1989 Annual Fall Meeting of the AIChE, San Francisco, CA (November 5-10, 1.989).

2. Yortsos, Y. C., and Chang, J. "Capillary Effects on Steady-State Flow in Heterogeneous Cores," Transport in Porous Media, 5, 399-420 (1990).

3. Satik, C., Parlar, M. and Yortsos, Y. C., "A. Study of Steady-State, Steam-Water Counterflow in Porous Media," Int. J. Heat Mass Transf., in press (1991).

4. Parlar, M., Zeybek, M., and Yortsos, Y. C., "Steady-State, Vapor-Liquid Concurrent Flow: Relative Permeabilities and End Effects," paper SPE 20054 presented at the 1990 California Regional Meeting, Ventura, CA (April 4-6, 1990).

5. Sahimi, M., and Yortsos, Y. C., "Flow of non-Newtonian Fluids in Porous Media," paper presented at the 5th IFP Research Conference, Arles, France (May 14-18, 1990).

6. Yortsos, Y.C., Satik, G., Bacri, J.C., and Salin, D., "Large Scale Averaging of Drainage at Local Capillary Control", submitted (1991).

7. Zeybek, M., and Yortsos, Y.C., "Long Waves in Parallel Flow in Hele-Shaw Cells", submitted (1991). 


\section{References}

[1] M.J. Ablowitz and H. Segur. Solitons and the Inverse Scattering Transform. SIAM, 1981.

[2] B. Amaziane and A. Bourgeat. Numerical Simulation in Oil Recovery. Springer Verlag, Now York, 1988. (M.F. Wheeler, editor).

[3] P. Armitage and R.A. Dawe. Paper SPE 18495 presented at the International Symposium on Oilfield and Geothermal Chemistry, Houston, TX, Feb. 8-10, 1989.

[4] J.L. Auriault, G. Lebaigue, and O. Bonnet. Transport in Porous Media, 4:105, 1989.

[5] J.-C. Bacri, M. Rosen, and D. Salin. Europhs. Lett., 11:127, 1990.

[6] M.F. Barnsley. Fractals Everywhere. Academic Press, Bostor, 1988.

[7] C. Barton and P. Hsieh. Physical and Hydrologic-Flow Properties of Fractures.Field Trip Guidebook T385. United States Geological Survey, 1989.

[8] J. Bear, C. Braester, and P. Menier. Transport in Porous Media, 2:301, 1987.

[9] D. Bensimon, L.P. Kadanoff, S. Liang, B.I. Shraiman, and C. Tang. Rev. Mod. Phys., 58:977, 1986.

[1.0] A. Bensoussan, J.L. Lions, and G. Papanicolaou. Asymptotic Analysis for Periodic Structurcs. North-Holland Publishing Company, Amsterdam, 1978.

(11) D.V. Boger. Ann. Rev. Fluid. Mech., 19:157, 1987.

[12] W.J. Canella, C. Huh, and R.S. Seright, Paper SPE 18089, presented at the 63rd Annual SPE Fall Meeting, Houston, TX, Sept. 1.988.

[13] K.T. Chambers and C.J. Radke. Interfacial phenomena in petroleum recovery. 1990. (N.R. Morrow, editor).

[14] J. Chang and Y.C. Yortsos. SPE Formation Evaluation, page 31, 1990.

[15] A. Chatenever and J.C. Calhoun, Jr. Trans. AIME, 195:149, 1952. 
(16) A. Chatenever, M.K. Indra, and J.R. Kyte. JPT, page 13, 1959.

17] R.H. Christopher and S. Middleman. Ind. Eng. Chem. Fund., 4:2122, 1965.

[18] K.H. Coat, J.R. Dempsey, and J.H. Anderson. SPEJ, page 63, 1971.

[19] R. Combescot, T. Dombre, V. Hakim, Y. Pomeau, and A. Pumir. Phys, Rev, Let., 56:2036, 1986.

[20] J.R. Counsil. Steam-Water Relative Permeability. PhD thesis, Stanford University, Stanford, 1979.

(21) A. Danesh, J.M. Peden, D. Krinis, and G.D. Henderson. Paper SPE 16956 presented at the 62nd Annual SPE Fall Meeting, Dallas, TX, September 27-30, 1987.

(22) P.G. de Gennes and E. Guyon. J. Mec., 17:403, 1978.

[23] M.M. Dias and D. Wilkinson. J. Phys. A., 19:3131, 1986.

[24] T.M. Doscher. Soaled physical model studies of the steam cirive process. Technical report, Univ. of Southern California, 1980.

(25] P.(x. Drazin and R.J. Johnson. Solitons: An Introduction. Cambridge University Press, 1989.

[26] F.A.L. Dullien. Porous Media, Fluid Transport and Pore Structure. Academic Press, New York, 1979.

27) F.J. Fayers and T.M.J. Newley. SPERE, page 542, 1988.

[28] J. Feder. Fractals. Plenum Press, 1988.

[29] B. Fornberg and G.B. Whitham. Proc R. Soc.Lond., 289:373, 1978.

[30] R.E. Garrison, editor. The Monterey Formation and Related Silicieous Rocks of California. The Pacific Section Society of Economic Paleontologists and Mineralogists, Los Angeles, 1981.

[31] J.F. Gouyet, M. Rosso, and B. Sapoval. Phys. Rev. B, 37:1832, 1988.

32) L.L. Handy. API Drilling and Production Practices, 1957. 
[33] A.A. Heiba, H.T. Davis, and L.E. Scriven. Paper SPE 12172 presented at the 58th Annual SPE Fall Meeting, San Francisco, CA, October 5-8, 1983.

[34] A.A. Heiba, M. Sahimi, L.E. Scriven, and H.T. Davis. Paper SPE 11015 presented at the 57th Annual SPE Fall Meeting, New Orleans, LA, Sept. 26-29, 1982.

[35] J. Heinrichs and N. Kumar. J. Phys. C: Solid State Phys., 8:L510, 1975.

[36] T.A. Hewett. Paper SPE 15386 presented at the 61st Annual SPE Fall Meeting, New Orleans, LA, Sept. 1986.

37] T.A. Hewett and R.A. Behrens. Paper SPE 20739 presented at the 65th Annual SPE Fall Mceting, New Orleans, LA, Sept. 23-26, 1990.

[38] G.M. Homsy. Ann. Rev. Fluid Mech., 19:271, 1987.

(39] V. Hornof and N.R. Morrow. SPERE, page 251, 1988.

(40) A.J. Katz and A.H. Thompson. J. Gesphys. Res., 92:599, 1987.

(41] S.W. Kenkel and J.P. Straley. J.P., Phys. Rev. Lett., 49:76', 1982.

[42] J. Kevorkian and J.D. Cole. Perturbation Methods in Applied Mathematics. Springler-Verlag, 1980.

43] M.J. King and H. Sher. Phys. Rev., A 41:874, 1990.

44] S. Kirkpatrick. Rev. Mod. Phys., 45:574, 1973.

[45] D.L. Fxch and J.F. Brady Jr. J. Fluid Mech., 180:387, 1987.

16) D.L. Koch and J.F. Brady Jr. Phys. Fluids, 31:1330, 1088.

17] J. Koplik. Fluid Mech., 119:219, 1982.

18. R.G. Larson. Ind. Eng. Chem. Funds., 20:132, 1981.

149j J. Lee, A Coniglio, and II.E. Stanley. Phys. Rev., A41:4587, 1990.

50) R. Lenormind. Proc. R. Soc. Lond., A 423:159, 1989. 
[51] R. Lenormand and B. Bories. C.R. Acad. Sci., Paris, 291B:279, 1980.

[52] R. Lenormand, E. Touboul, and C. Zarcone. J. Fluid Mech, 189:165, 1988.

[53] T. Maxworthy. Phys. Rev. A, 39:5863, 1989.

[54] F.G. Miller. Trans. AIME, 192:205, 1951.

[55] K. Mohanty. In The 5th IFP Research Conference, Arles, France, May 14-18, 1990.

[56) A. Monsalve, R.S. Schechter, and W.H. Wade. Paper SPE 12661 presented at the 1984 SPE/DOE Symposium on Enhanced Oil Recovery, T́ulsa, OK, April, 1984.

57] D. Nolte, L. Pyrak-Nolte, and G. Cook. PAGEOPH, 131:111, 1989.

[58] S. Owete and E. Brigham. SPE Reservoir Engineering, page 315, 1987.

[59] C.W. Park and G.M. Homsy. J. Fluid Mech, 139:291, 1984.

60] M. Parlar and Y.C. Yortsos. Paper SPE 16969 presented at the 62nd Annual SPE Fall Meeting, Dallas, TX, Sept. 27-30, 1987.

[01] M. Parlar and Y.C. Yortsos. J. Colloid Interface Sci., 124:162, 1988.

(62] M. Parlar and Y.C. Yortsos. J. Colloid Interface Sci., 132:425, 1989.

(63) L. Paterson. J. Fluid Mech., 113:513, 1981.

64) P. Pelcé. Dynamics of Curved Fronts. Academic Press, 1988.

65] M.S. Pless and A. Prosperetti. Ann. Rev. Fluid Mech., 9:145, 1977.

66j M.S. Plesset and S.A. Zwick. J. Appl. Phys., 25:493, 1954.

(67) K. Pruess. Annual Report, Earth Sc. Div., Lawrence Berkeley Laobratory, Berkeley, CA, 1982.

(8) M. Quintard and S. Whitaker. Transport in Porous Media, 3:357, 1988.

69) M. Quintard and S. Whitaker. Transport in Porous Media, 5:341, 1990. 
[70] W.R. Rossen and P.A. Gauglitz. AIChEJ, 36:1176, 1990.

[71] M. Rosso, J.F. Gouyet, and B. Sapoval. Phys. Rev. B, 32:6053, 1985.

[72] M. Rosso, J.F. Gouyet, and B. Sapoval. Phys. Rev. Lett., 57:3195, 1986.

(73) P.G. Saffman and G.I. Taylor. Proc. Roy. Soc. London Ser., A245:312, 1958.

[74] M. Sahimi, G.R. Gavalas, and T.T. Tsotsis. Chem. Eng. Sci., 45:1443, 1990.

[75] M. Sahimi and Y.C. Yortsos. Paper SPE 20476 presented at the 65th Annual SPE Fall Meeting, New Orleans, LA, Sept. 23-26, 1990.

[76] M. Salman, S. Baghdikian, L.L. Handy, and Y.C. Yortsos. submitted. SPERE, 1989.

(77) C. Sammis, G. King, and R. Biegel. PAGEOPH, 125:777, 1987.

[78] J.M. Sanchez and Schechter R.S. Paper SPE 16967 presented at the 62nd Annual SPE Fall Meeting, Dallas, TX, Sept. 27-30, 1987.

[79] B. Sapoval, M. Rosso, and J.F. Gouyet. J. Phys. (Paris) Lett., 46:L149, 1985.

[80] C. Satik, M. Parlar, and Y.C. Yortsos. Int. J. Heat Mass Transf. in press, 1991.

[81] A.E. Scheidegger. The Physics of Flow Through Porous Media. University of Toronto Press, Toronto, 1974.

[82] L.E. Scriven. Chem. Eng. Sci., 10:1, 1959.

[83] M.M. Sharma and Yortsos Y.C. AIChEJ, 33:1644, 1987.

184] A.K. Singhal. Physical model study of inverted seven-spot steam floods in a pool containing a lloydminster type heavy oil. Technical report, Petroleurn Research Institute, Canada, 1978.

[85] K.S. Sorbie, P.J. Clifford, and E.R.W. Jones. J. Coll. Interf Sci., 130:508, 1989.

(86)] D. Stauffer. Introduction to Percolation Theory. Francis-Taylor, 1985.

87] G.L. Stegmeier, D.D. Laumbach, and C.W. Volek. Paper SPE 6787 preseliced at the 52nd SPE Annual Fall Meeting, Denver, CO, Oct. 9-12, 1977. 
[88] R.B. Stinchcombe. J. Phys. C: Solid State Phys., 7:179, 1974.

[89] J.P. Straley and S.W. Kenkel. Phys. Rev. B, 29:6299, 1984.

(90) J. Szekely and G.P. Martins. Chem. Eng. Sci., 26:147, 1971.

(91) D. Teeuw and F. Hesselink. Paper SPE 8982 presented at the 5 th International Symposium on Oilfield and Geothermal Chemistry, Stanford, CA, May 28-30, 1980.

192] A.E. Trimble and D.E. Menzie. Paper SPE 5571 presented at the 50th Annual SPE Fall Meeting, Dallas, TX, Sept. 1975.

[93] G. Tryggvason and H. Aref. J. Fluid Mech., 136:1, 1983:

[94] P. van Meurs. Trans. AIME, 210:295, 1959.

[95] A. Verma and K. Pruess. Proc. Eleventh Workshop on Geothermal Reservoir Engineering, Stanford Univ., Stanford CA, Jan. 1986.

[96] A.K. Verma. PhD thesis, University of California, Berkeley, 1986.

[97] J.E. Warren and P.P. Root. SPEJ, page 245, 1963.

(98) S. Whitaker. Transport in Porous Media, 1:105, 1986.

(99) G. B. Whitham. Linear and Nonlinear Waves. John Wiley, 1974.

100) D Wilkinson. Phys. Rev. A, 34, (1986).

[101] D. Wilkinson and J.F. Willemsen. J. Phys. A, 16:3365, 1983.

[102] R.A. Wooding. J.Fluid Mech., 7:501, 1960.

[103] Y.C. Yortsos and J. Chang. Paper SPE 18798 presented at the 1989 California Regional Meeting, Bakersfield, CA, 1989.

(i).4) Y.C. Yortsos and J. Chang. Transport in Porous Media, 5:399, 1990.

(105) Y.C. Yortsos and M. Parlar. Paper SPE 19697 preserited at 64th Annual SPE Fall Mecting, San Antonio, TX, October 8-11, 1989. 
[106] Y.C. Yortsos and M. Sharma. A[ChEJ, 32:46, 1986.

[107] V.J. Zapata and L.W. Lake. F'aper SPE 10111 presented at the 56th Annual SPE Fall Meeting, San Antonio, TX, Oct. 5-7, 1981. 


\section{APPENDIX}

Upon manipulation of equations, the following system is obtained in dimensionless notation

$$
\begin{aligned}
\frac{d J}{d S} \frac{d S}{d \xi} & =R_{m} \frac{F(\Theta, S ; \xi)}{H(\Theta, S ; \xi)} \\
\frac{d \Theta}{d \xi} & =R_{m} \frac{G(\Theta, S)}{H(\Theta, S ; \xi)}
\end{aligned}
$$

where

$$
\begin{aligned}
F(\Theta, S ; \xi) \equiv & {\left[\frac{K R_{p} A}{\Theta^{2}} k_{D}^{1 / 2}\left(W-\frac{E}{R_{m}}\right)\right.} \\
& +\frac{R_{L}\left(1+b R_{p} A\right) J}{2 k_{D}} \frac{d k_{D}}{d \xi} \\
& \left.-\frac{K R_{p} A J W}{2 R_{m} \Theta^{2}} \frac{d k_{D}}{d \xi} R_{\mu} R_{\rho} k_{r v}\right] k_{r} L \\
& +\left[\frac{K R_{p} A}{\Theta^{2}} k_{D}^{1 / 2}\left(-\frac{E}{R_{m}}\right)\right. \\
& \left.+\frac{R_{L} b R_{p} A J}{2 k_{D}} \frac{d k_{D}}{d \xi}\right] R_{\mu} R_{\rho} k_{r v} \\
& +\frac{R_{h}}{k_{D}^{1 / 2}} \\
& \left(1+b R_{p} A\right)\left(W-\frac{E}{R_{m}}\right) k_{r L} \\
& +b R_{p} A\left(-\frac{E}{R_{m}}\right) R_{\mu} R_{\rho} k_{r v} \\
G(\Theta, S) \equiv & {\left[R_{h}\left(1+b R_{p} A\right)\right.} \\
& \left.-\frac{K R_{p} A}{\Theta^{2}} k_{D} W R_{\mu} R_{\rho} k_{r v}\right] k_{r L} \\
& +R_{h} b R_{p} A R_{\mu} R_{\rho} k_{r v}
\end{aligned}
$$

The various dimensionless terms are defined in Table 2. Important variables in what follows are $R_{m}$ and $h_{h}$, the first denoting a macroscopic capillary number and the latter expressing the conduction effect.s. Parameter $b(\ll 1)$ is a measure of Kelvin effects, typically negligible, except at low values of the liquid saturation $S$. Variables $W-E / R_{m}$ and $-E / R_{m}$ represent local vapor and liquid fraction 


$$
\begin{aligned}
W-\frac{E}{R_{m}} & =\frac{h_{L}(T)-C / m_{i}}{L_{v r}} \\
-\frac{E}{R_{m}} & =\frac{h_{v}(T)-C / m_{i}}{L_{v r}}
\end{aligned}
$$

respectively. It must be noted that these are the only parameters that carry information about the injected enthalpy rate, $C$. Under isothermal conditions they simplify to

$$
\begin{aligned}
W-\frac{E}{R_{m}} & =-\chi_{0} \\
-\frac{E}{R_{m}} & =1-\chi_{0}
\end{aligned}
$$

where $\chi_{0}$ is the steam quality at injection. Finally, the dimensionless vapor pressure $A$, normalized with respect to the saturation pressure at the reference temperature is given by

$$
A=\exp \left[K\left(1-\frac{1}{T}\right)-\frac{b J(S)}{\sqrt{k_{D}(\xi)}}\right]
$$



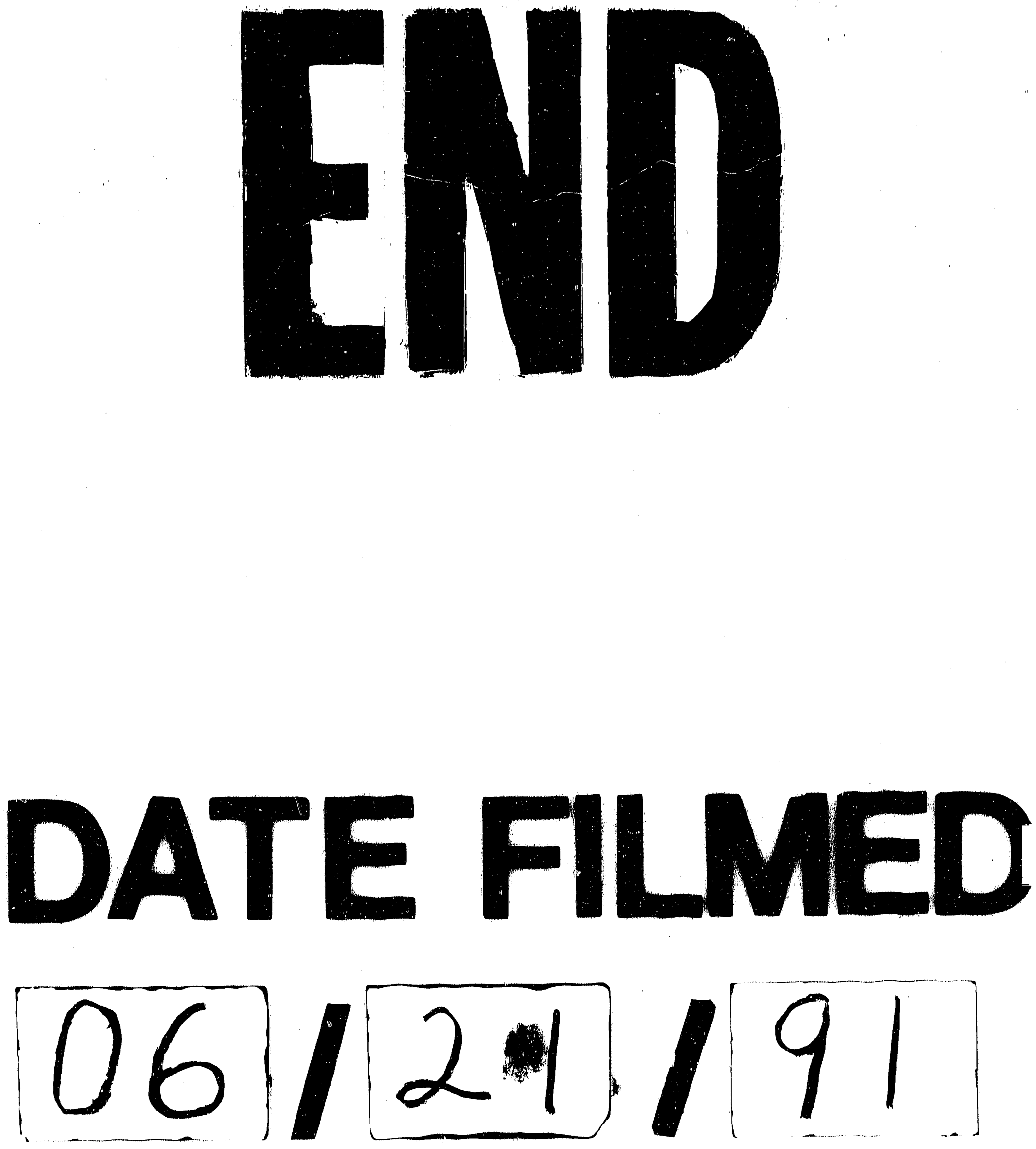
